\title{
Analysis of Gas-Liquid Flow in an Aerated Reactor Equipped with a Coaxial Mixer through Tomography and CFD
}

\author{
By \\ Nasim Hashemi \\ M.Sc. Sharif University of Technology, Tehran, Iran, 2007 \\ B.Sc. Arak University, Arak, Iran, 2002 \\ A dissertation \\ presented to Ryerson University \\ in partial fulfillment of the requirements for the degree of \\ Doctor of Philosophy \\ in the program of Chemical Engineering
}

Toronto, Ontario, Canada, 2017

(C) Nasim Hashemi 2017 


\section{$\underline{\text { Author's Declaration }}$}

I hereby declare that I am the sole author of this dissertation. This is a true copy of the dissertation, including any required final revisions, as accepted by my examiners.

I authorize Ryerson University to lend this dissertation to other institutions or individuals for the purpose of scholarly research.

I further authorize Ryerson University to reproduce this dissertation by photocopying or by other means, in total or in part, at the request of other institutions or individuals for the purpose of scholarly research.

I understand that my dissertation may be made electronically available to the public. 


\section{ABSTRACT}

\section{Nasim Hashemi}

\section{Analysis of Gas-Liquid Flow in an Aerated Reactor Equipped with a Coaxial Mixer through Tomography and CFD}

\section{PhD, Chemical Engineering, Ryerson University, Toronto, 2017}

This doctoral thesis addresses the mixing of higly viscous Newtonian fluids (corn syrup solutions) in a novel aerated reactor equipped with a central impeller (a pitched blade turbine in upward or downward pumping mode) and a wall scraping anchor. The non-intrusive electrical resistance tomography (ERT), dynamic gas disengagement method (DGD), design of experiments (DOE), computational fluid dynamics (CFD), and population balance model (PBM) were employed to characterize the performance of this novel aerated system. The performance criteria to be examined were mixing time, power uptake, gas holdup, and bubble size distribution.

In this study, novel correlations were developed to estimate the gassed power drawn by the coaxial mixer, mixing time, and gas holdup. In addition, to obtain a master power curve, two new dimensionless correlations were proposed for the generalized power number and gas flow number by incorporating the equivalent rotational speed for the coaxial mixer, speed ratio (central impeller speed/anchor speed), and the central impeller power fraction into these two correlations. The experimental data demonstrated that gas flow affected the aerated anchor power consumption and central impeller power consumption in different manners. It was also found that at the higher fluid viscosity and beyond the critical speed ratio of 10 , the anchor power consumption was increased 
by increasing the speed ratio (i.e. decreasing the anchor speed). It was shown that in the presence of gas, the anchor impeller in combination with the upward pumping pitched blade turbine in the co-rotating mode exhibited shorter mixing times and lower power consumption than the anchordownward pumping pitched blade coaxial mixer.

To enhance the efficiency of the aerated mixer, it is critical to investigate the influence of the gasliquid flow within the vessel on the bubble size distribution (BSD) and the local and global gas holdup. To achieve this goal, the effects of the bubble breakup and coalescence on the BSD within the vessel were incorporated into the CFD model through the CFD-PBM coupling. The experimental and simulation results showed that beyond the critical speed ratio of 10 , the volume fractions of the large bubbles decreased while the volume fractions of the small bubbles increased. 


\section{Acknowledgments}

It is a genuine pleasure to express my deep sense of thanks and gratitude to my supervisor, Professor Farhad Ein-Mozaffari, for his competent and motivating guidance, innovative ideas, and stoic patience. His ideas and enthusiasm have always inspired me to solve critical issues in my research and overcome many experimental and numerical obstacles His technical and nontechnical advice was invaluable to me in my professional and personal life. I am always indebted to him for sharing his wealth of knowledge with me. In spite of his busy schedule, he was always available to clarify my doubts.

I would like to thank my co-supervisors, Professor Simant R. Upreti and Professor Dae Kun Hwang for their expert help, guidance, constructive criticism as well as their positive encouragement.

I would like to thank my committee members Professor Huu Doan, Professor Krishnan Venkatakrishnan, Professor Mita Ray, and Professor Philip Chan for their precious time and consideration.

I acknowledge the assistance and great help of the administration staffs, Mrs. Alanna Mcknight, Mrs. Isabella Fernandes, and Mrs. Louise Lichacz, and also engineering staff, Mr. Ali Hemmati, Mr. Daniel Boothe, and Mr. Tondar Tajrobekar, in the Chemical Engineering Department at Ryerson University.

I also would like to thank all my friends who encouraged me in every aspect during the project. Special thanks to Dr. Zoya Bahreini for being always beside me in happiness and sorrow, in my success and failures.

I acknowledge Natural Sciences and Engineering Research Council of Canada (NSERC) and Ryerson Graduate Scholarship for the financial support during this work. I also acknowledge the HPCVL (High Performance Computing Virtual Laboratory) for providing the high performance computing facilities.

I am forever indebted to my family for their understanding, endless patience and encouragement from the beginning. Thanks for all the calls and visits that inspired and motivated me to carry on. 
Also, thanks to my lovely husband, Iman Alizadeh, who stood by my side during the ups and downs, for believing in me and for supporting my ideas and dreams.

Finally, thanks from the depths of my soul and my heart to my baby, Soren, for being so great and patient. 


\section{This dissertation is dedicated to the following people:}

The memory of my father, M. Hashemi, who did everything he could to enable me to get a good education. He always encouraged me to strive for excellence.

To my mother, N. Bayat, who has been my number one support and friend throughout my education. She brought me up under very difficult circumstances.

To my siblings who have been my role models and inspired me to achieve my goals.

To my beloved husband who has offered unwavering support and encouragement during the past two years of my doctoral journey.

To my little son, Soren, whose bright eyes and smile encouraged me to pursue my doctoral research. 


\section{Table of contents}

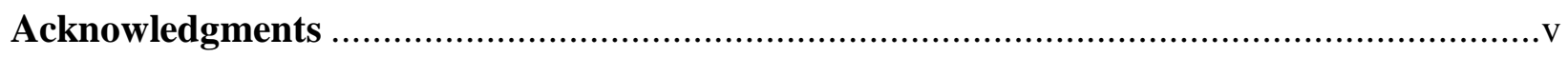

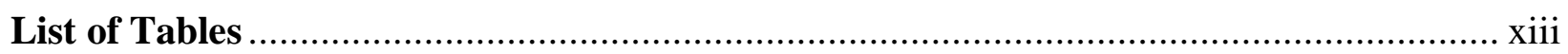

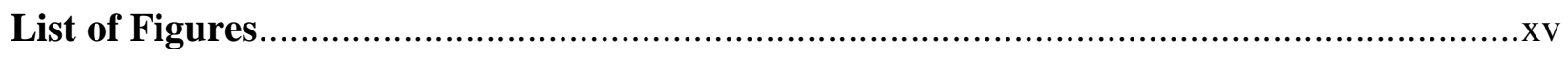

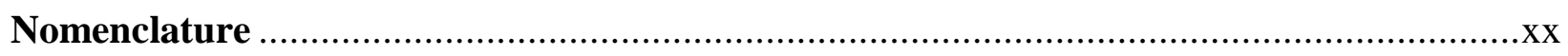

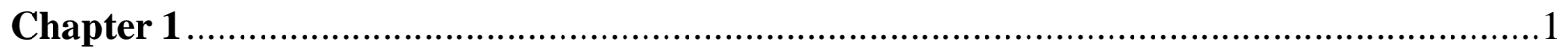

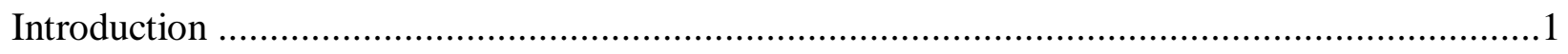

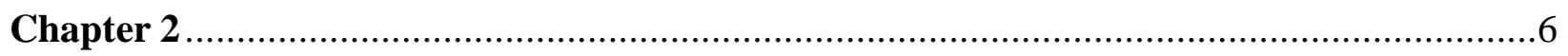

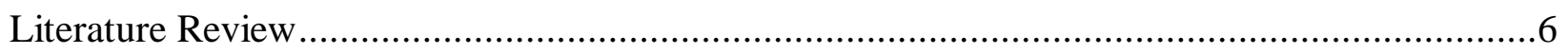

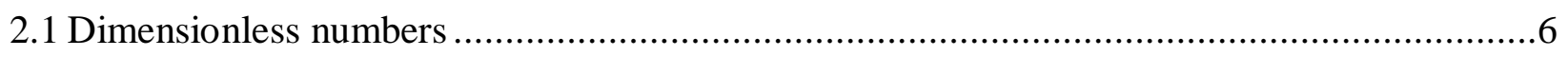

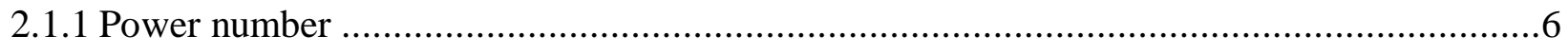

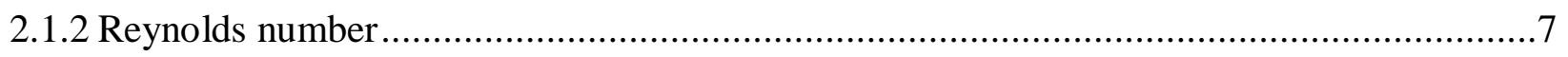

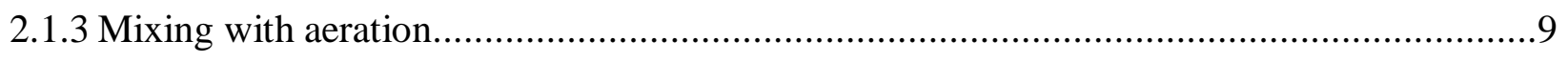

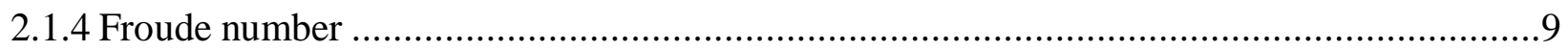

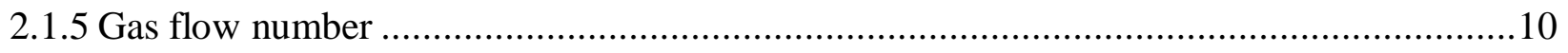

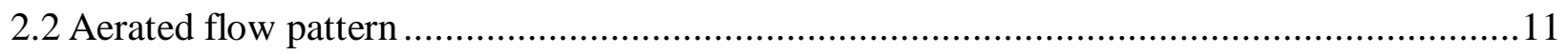

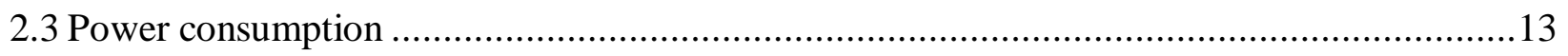

2.3.1. Power requirement for gas-liquid stirred vessel............................................................ 14

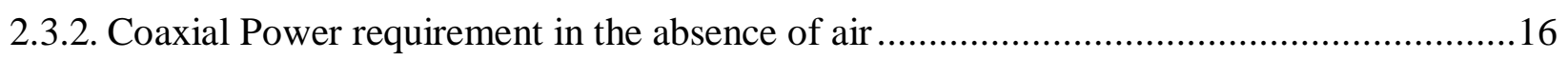

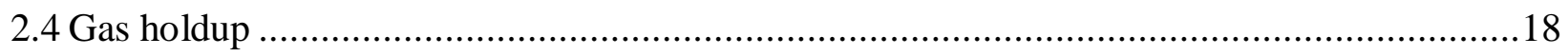

2.4.1. Gas holdup in single and multi-impeller configurations ................................................18

2.4.2. Effect of liquid viscosity on the gas holdup ...............................................................19

2.4.3. Proposed correlations for measuring gas holdup ....................................................... 19 


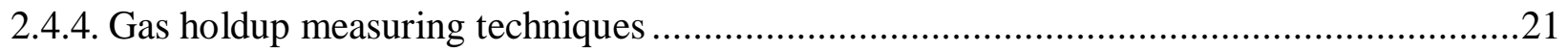

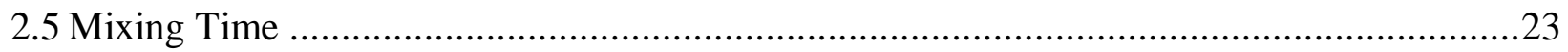

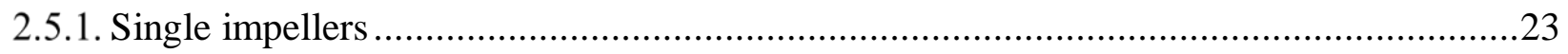

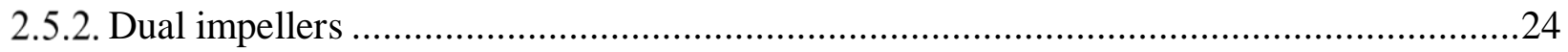

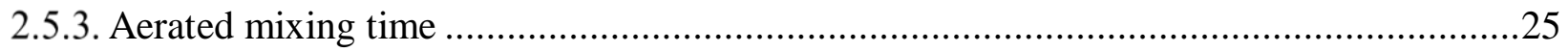

2.5.4. Mixing time measurement techniques ...............................................................27

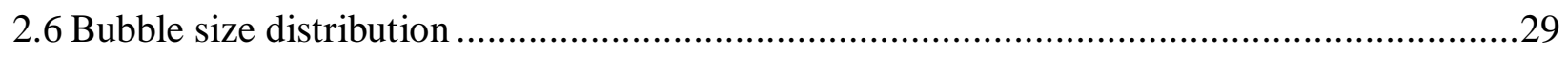

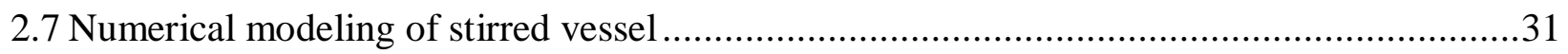

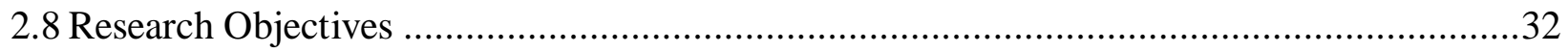

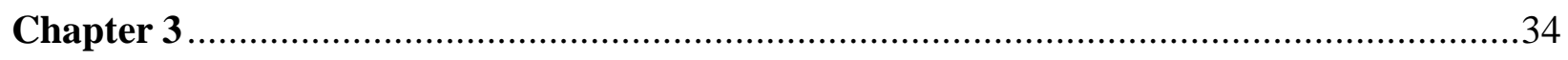

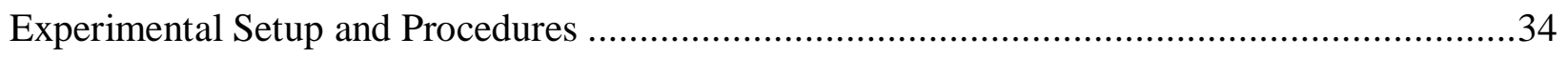

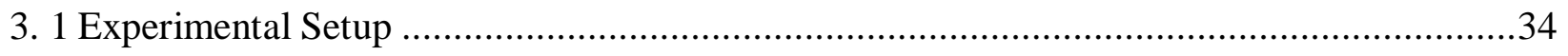

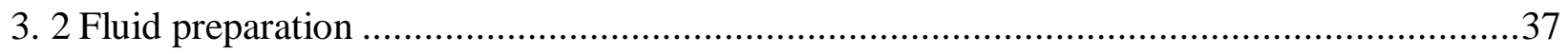

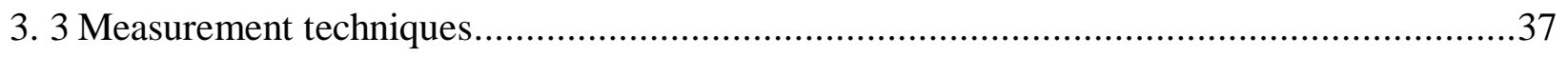

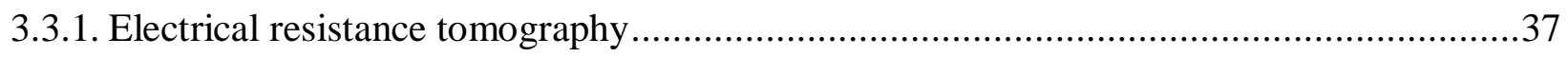

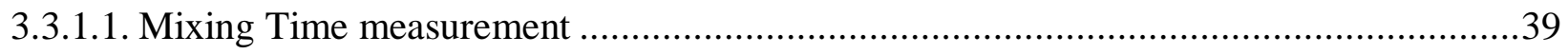

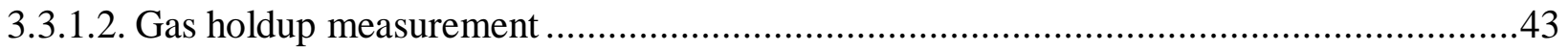

3.3.1.3. Dynamic Gas Disengagement Technique procedure ..........................................46

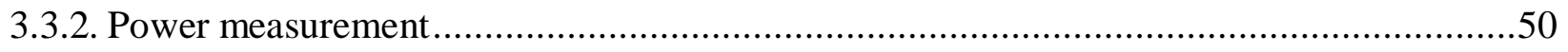

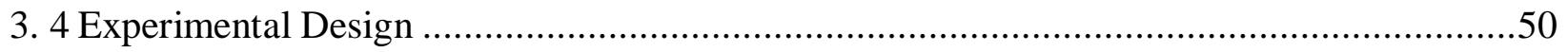

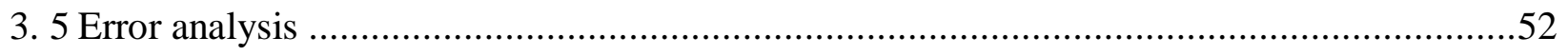

3.5.1. Evaluation of Torque Sensor Precision ........................................................52

3.5.2. Evaluation of ERT Measurements Precision ..................................................52 


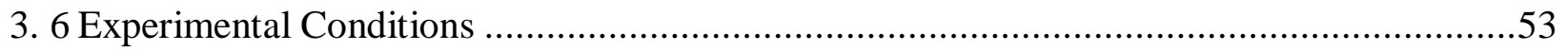

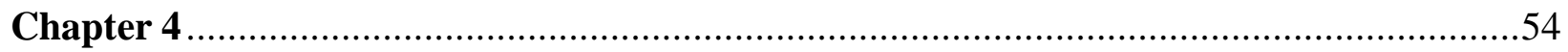

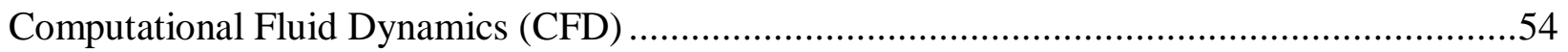

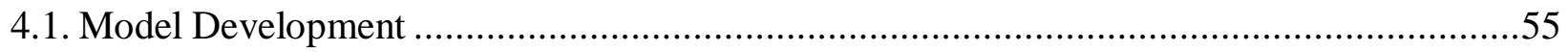

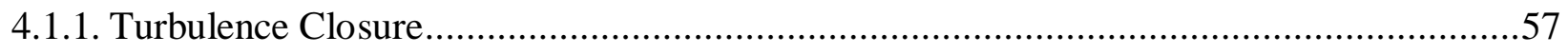

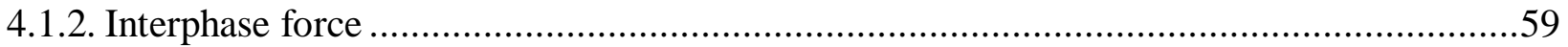

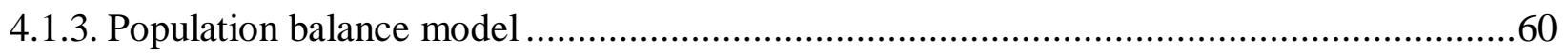

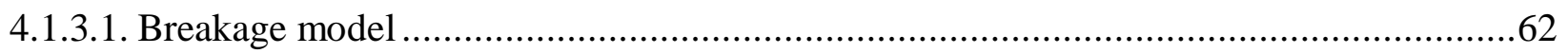

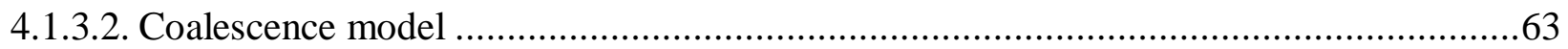

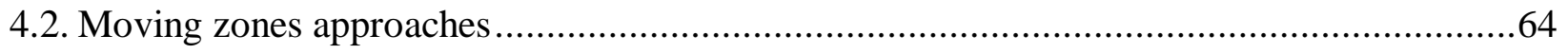

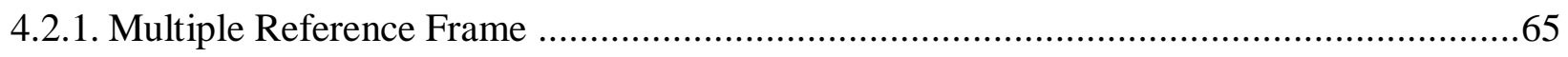

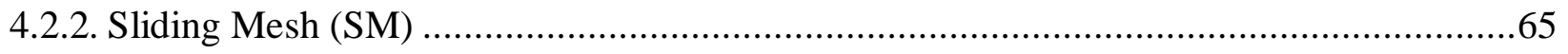

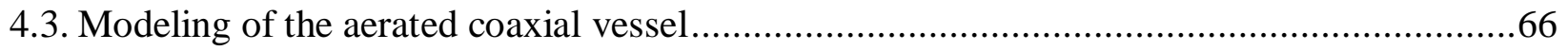

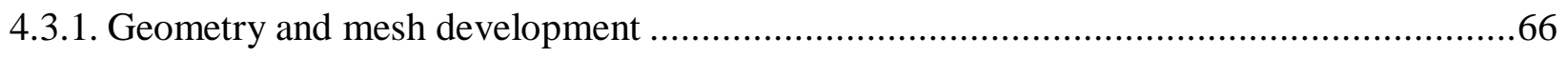

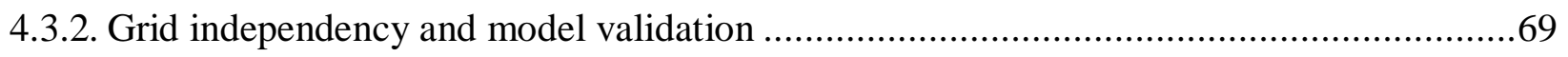

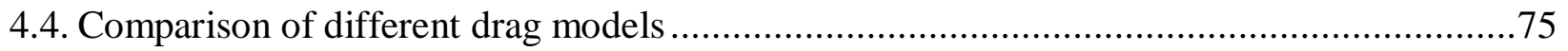

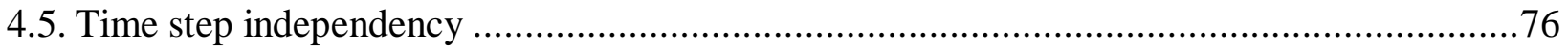

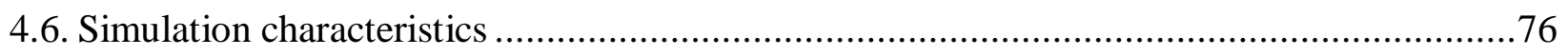

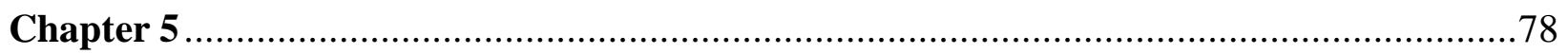

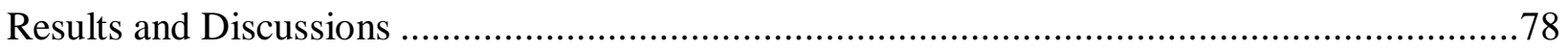

5.1. Analysis of mixing in an aerated reactor equipped with the coaxial mixer through electrical

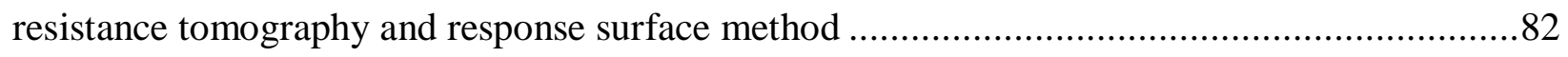

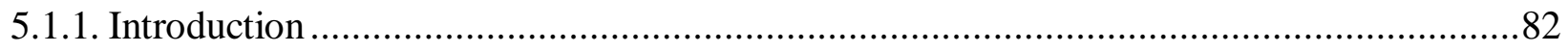

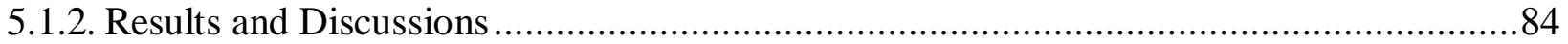




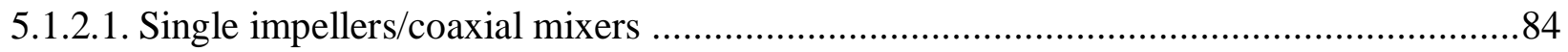

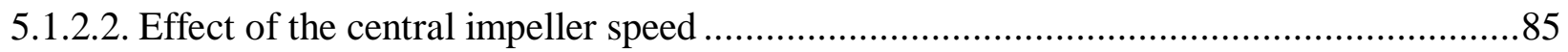

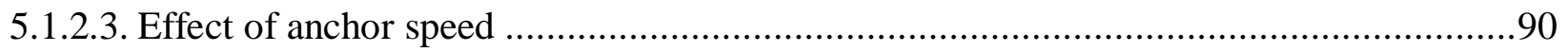

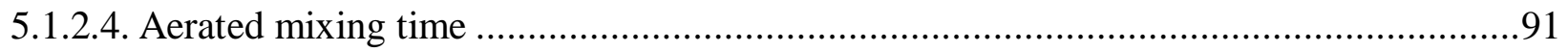

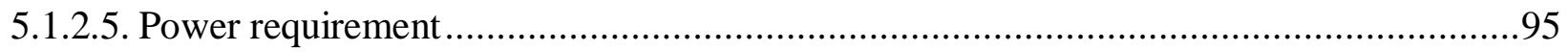

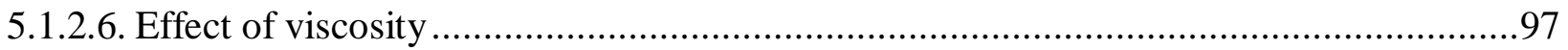

5.1.2.7. Response surface methodology model development.................................................... 100

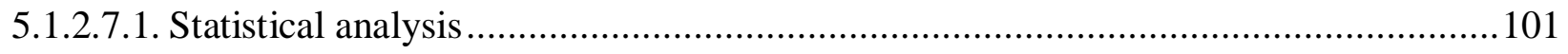

5.1.2.7.2. Analysis of the model coefficient and their interactions.......................................... 102

5.1.2.7.3. Three-dimensional response surfaces ……............................................................ 103

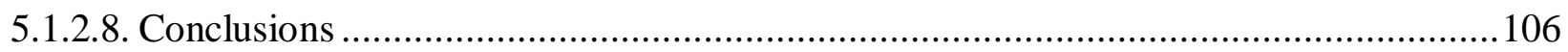

5.2. Analysis of power consumption and gas holdup distribution for an aerated reactor equipped with a coaxial mixer: Novel correlations for the gas flow number and gassed power ................107

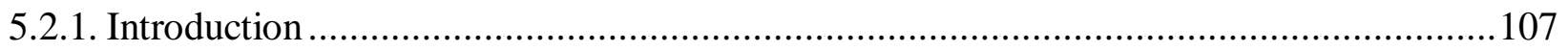

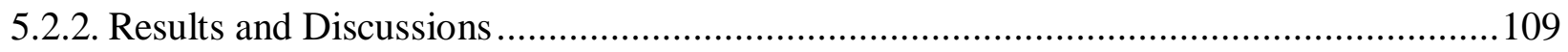

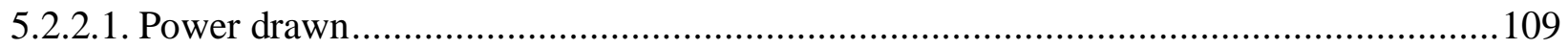

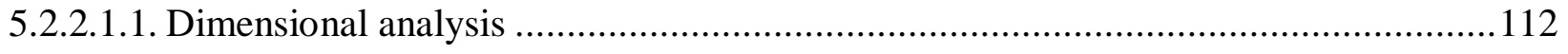

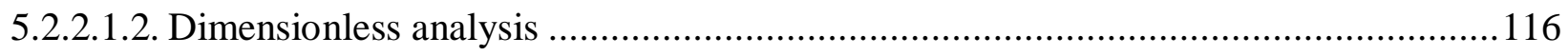

5.2.2.2. Radial gas holdup distribution in the aerated coaxial mixing vessel............................ 124

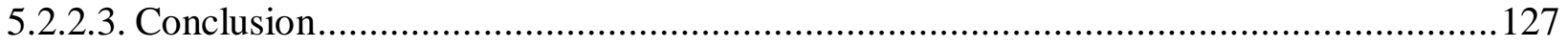

5.3. Experimental investigation of the bubble behavior in an aerated coaxial mixing vessel

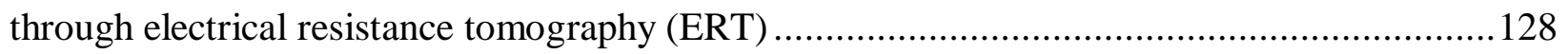

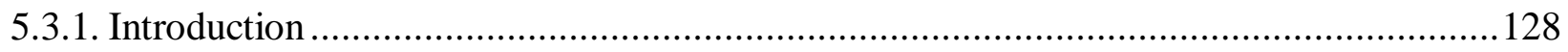

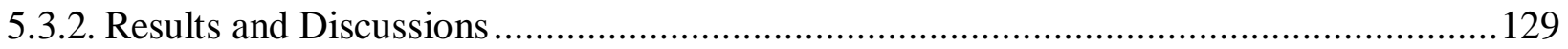


5.3.2.1. Local and overall gas holdup of bubbles with different sizes

5.3.2.2. Gas holdup correlation for the aerated coaxial mixing vessel

5.3.2.3. Effect of the speed ratio on the Sauter mean bubble diameter in the aerated coaxial mixing vessel

5.3.3. Conclusion

5.4. Hydrodynamic characteristics of an aerated coaxial mixing vessel equipped with a pitched blade turbine and an anchor

5.4.1. Introduction 143

5.4.2. Results and Discussions 144

5.4.2.1. Effect of the speed ratio on the gas holdup at different syrup viscosities.

5.4.2.2. Effect of rotation mode on the gas holdup

5.4.2.3. Flow field 155

5.4.2.4. Conclusion.

5.5. Modeling of bubble size distribution in a bioreactor equipped with a coaxial mixer using population balance model coupled with CFD

5.5.1. Introduction 158

5.5.2. Results and discussions 159

5.5.2.1 Bubble size distribution 159

5.5.2.2 Effect of speed ratio on the bubble size distributions 163

5.5.2.3 Conclusion 171

Chapter 6 173

Conclusions 173

6.1 Recommendations for Future Work 178

References. 179 


\section{List of Tables}

Table (2.1 - 1). $K_{p}$ values for different impellers ........................................................................8

Table (2.2- 1). Proposed correlations for predicting the transition between different flow patterns developed in the gas-liquid mixing vessels.

Table (2.3- 1). Several correlations for estimating power consumption in the presence of gas...15

Table (2.3- 2). Different correlations offered by various authors for Reynolds number and power number of coaxial mixers. .......................................................................................................17

Table (2.4- 1). Correlations for gas holdup estimation.............................................................20

Table (2.5- 1). Mixing time correlations for single and multi-impeller systems...........................26

Table (2.5- 2). Advantages and disadvantages of mixing time measurement techniques.............27

Table (3.3- 1). Tomography parameters applied in this study...........................................39

Table (3.4- 1). Independent variables and their actual values using Box-Behnken design..........51

Table (3.4- 2). Model summery statistics for mixing time.

Table (3.6- 1). Employed parameters in the current work........................................................53

Table (4.4- 1). Comparison of different drag models................................................................75

Table (5.1- 1). Four factor Box-Behnken design showing independent variables level and the observed and predicted mixing times......................................................................100

Table (5.1 - 2). Analysis of variance for response surface quadratic model .............................102

Table (5.2- 1). Equivalent rotational speed, $R e$, and $\mathrm{N}_{\mathrm{p}}$ proposed for coaxial mixers...............110

Table (5.2- 2). Regression constants of the gassed power correlation proposed for the coaxial mixing vessel equipped with PBU-anchor and PBD-anchor. .115 
Table (5.3- 1). The experimental conditions. ................................................................129

Table (5.3- 2). Different published correlations for the prediction of gas holdup in agitated vessels ..................................................................................................................................................136

Table (5.5- 1). Different correlations for the bubble size at the sparger.

Table (5.5- 2). Effect of the speed ratio on the volume fractions of the small size bubbles in the upper and bottom compartments of the coaxial mixer....................................................171 


\section{List of Figures}

Figure (2.2- 1). Gas distribution patterns in the aerated vessel equipped with disc turbine as a function of impeller speed and gas flow rate (John, 1998).

Figure (3.1- 1). Experimental set-up (dimensions in $\mathrm{mm}$ ) .36

Figure (3.3- 1). The arrangement of the electrical resistance tomography sensor planes. 38

Figure (3.3- 2). Mean Conductivity data versus time for corn syrup solution agitated by PBUanchor aerated coaxial mixer at $N_{a}=10 \mathrm{rpm}, N_{c}=90 \mathrm{rpm}$ and gas flow rate $0.0094 \mathrm{~m}^{3} / \mathrm{min}$ using ERT.

Figure (3.3- 3). 2D, 3D, and stacked tomography images showing the level of tracer homogeneity for corn syrup solution agitated by PBU-anchor at $\mathrm{Re}=1757$ and gas flow rate $0.0283 \mathrm{~m}^{3} / \mathrm{min}$ (Plane 1: $(\mathrm{z}=0.314 \mathrm{~m})$, Plane 2: $(\mathrm{z}=0.258 \mathrm{~m})$, Plane 3: $(\mathrm{z}=0.202 \mathrm{~m})$, Plane 4: $(\mathrm{z}=0.146 \mathrm{~m})$, and Plane 5: $(\mathrm{z}=0.090 \mathrm{~m}))$..................................................................................................42

Figure (3.3- 4). Experimental setup for measuring gas holdup using ERT...................................44

Figure (3.3- 5). Tomograms showing the conductivity distributions within the aerated PBU-anchor coaxial mixer: $=N_{c}=180 \mathrm{rpm}, \boldsymbol{N}_{\boldsymbol{a}}=10 \mathrm{rpm}, \boldsymbol{Q}_{g}=0.0283 \mathrm{~m}^{3} / \mathrm{min}$, and $\mu=1.4 \mathrm{~Pa} \mathrm{~s}$ .46

Figure (3.3- 6). Gas disengagement profiles of the aerated coaxial mixing vessel at $\boldsymbol{N}_{\boldsymbol{c}}=150 \mathrm{rpm}$ $N_{a}=9.4 \mathrm{rpm}$, and $\boldsymbol{u}_{g}=0.15 \mathrm{~m} / \mathrm{s}:$ (a) plane $2(z=0.258 \mathrm{~m})$ and (b) plane $4(z=0.146 \mathrm{~m}) \ldots . . . . .48$

Figure (3.3- 7). Tomograms showing the level of the gas dispersion for corn syrup solution agitated by PBU-anchor at $N_{c}=150 \mathrm{rpm}, N_{a}=15 \mathrm{rpm}$, and $\boldsymbol{u}_{g}=0.15 \mathrm{~m} / \mathrm{s}$.

Figure (4.3- 1). Developed model for coaxial vessel using Design Modeller. ............................67

Figure (4.3- 2). Mesh structure for central impeller......................................................................68

Figure (4.3- 3). Surface mesh for central impeller and anchor...................................................68

Figure (4.3- 4). Surface mesh developed for the vessel. ...............................................69

Figure (4.3- 5). Specified lines for grid independence study. ......................................................71

Figure (4.3- 6). Effect of the number of grids on the (a) axial velocity and (b) tangential velocity in horizontal position close to the central impeller [Line 1 in Figure (4.3-5)]..............................72

Figure (4.3- 7). Effect of the number of grids on the (a) tangential velocity, and (b) radial velocity

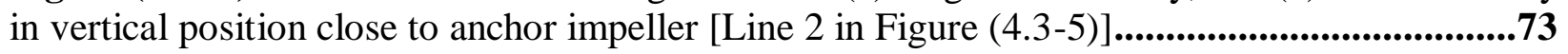

Figure (4.3- 8). Effect on grid on the gas holdup distribution......................................................74 
Figure (5.1- 1). Mixing time versus Reynolds number for the coaxial mixers in the co-rotating mode and single impellers s $(\max \mathrm{STDEV} \approx 1.8 \%)$.

Figure (5.1- 2). Mixing time versus Reynolds number when only the central impeller speed changes at constant gas flow rate of $0.0283 \mathrm{~m}^{3} / \mathrm{min}$ and the anchor speed of (a) $10 \mathrm{rpm}$, (b) $20 \mathrm{rpm}$, and (c) $30 \mathrm{rpm}(\max \mathrm{STDEV} \approx 1.69 \%)$.

Figure (5.1- 3). Mixing time versus Reynolds number as a function of the anchor speed at constant gas flow rate of $0.0283 \mathrm{~m}^{3} / \mathrm{min}$ for the coaxial mixing system equipped with (a) PBU-anchor and (b) PBD-anchor impellers (max STDEV $2.23 \%$ ).

Figure (5.1- 4). Mixing time versus Reynolds number when only the anchor speed changes at the constant central impeller speed of $135 \mathrm{rpm}$ and the fixed gas flow rate of $0.0283 \mathrm{~m}^{3} / \mathrm{min}$ for the PBU-anchor and the PBD-anchor coaxial mixers for both co-rotating and counter-rotating modes $(\max \mathrm{STDEV} \approx 4.76 \%)$. .91

Figure (5.1- 5). Mixing time versus gas flow number at the anchor speed of $10 \mathrm{rpm}$ and the central impeller speed of $135 \mathrm{rpm}(\max \mathrm{STDEV} \approx 1.1 \%)$.

Figure (5.1- 6). Effect of gassing on the mixing time at the anchor speed of $10 \mathrm{rpm}$ and the central impeller speed of (a) PBD (90 rpm) (max STDEV $\approx 4.16 \%)$, (b) PBU (90 rpm) (max $\mathrm{STDEV} \approx 2.08 \%)$, (c) $\mathrm{PBD}(180 \mathrm{rpm})(\max \mathrm{STDEV} \approx 1.16 \%)$, and (d) PBU (180 rpm) (max $\mathrm{STDEV} \approx 4.13 \%)$.

Figure (5.1- 7). Central impeller power contribution into the total gassed power of the aerated coaxial mixing vessel at constant gas flow rate $0.0283 \mathrm{~m}^{3} / \mathrm{min}$ and viscosity 0.7 Pa.s: (a) PBDAnchor, (b) PBU-Anchor.

Figure (5.1- 8). Power number vs. Reynolds number for the aerated BPU-anchor and PBD-anchor: (a) and (c) medium and high viscosities at gas flow rate $0.0189 \mathrm{~m}^{3} / \mathrm{min}$, (b) and (d) medium and high viscosity at gas flow rate $0.0283 \mathrm{~m}^{3} / \mathrm{min}$.

Figure (5.1- 9). Mixing time vs. power consumption of the aerated coaxial mixing vessel containing corn syrup solutions with the viscosities of 0.05 and $1.4 \mathrm{~Pa} . \mathrm{s}$ ( $\max \mathrm{STDEV} \approx 5 \%$ ).....99 
Figure (5.1- 10). Effect of gassing on the mixing time of the PBU-anchor and PBD-anchor coaxial mixers $\left(N_{c}=180 \mathrm{rpm}\right.$ and $\left.N_{a}=10 \mathrm{rpm} \mu=1.4 \mathrm{~Pa} . \mathrm{s}\right)(\max \operatorname{STDEV} \approx 2.17 \%)$.

Figure (5.1- 11). Reference surfaces showing mixing time as a function of two independent variables: (a) Central impeller speed $\left(X_{1}\right)$ and anchor speed $\left(X_{2}\right)$, (b) central impeller speed $\left(X_{1}\right)$ and gas flow rate $\left(X_{3}\right)$, (c) anchor speed $\left(X_{2}\right)$ and gas flow rate $\left(X_{3}\right),(\mathrm{d})$ anchor speed $\left(X_{2}\right)$ and viscosity $\left(X_{4}\right)$, (e) central impeller speed $\left(X_{1}\right)$ and viscosity $\left(X_{4}\right)$, and (f) gas flow rate $\left(X_{3}\right)$ and viscosity $\left(X_{4}\right)$

Figure (5.2- 1). Power curve of the PBU-anchor coaxial mixer under constant aeration, 0.0283 $\mathrm{m}^{3} / \mathrm{min}$, based on different correlations: (a) Foucault et al. (2006), (b) Farhat et al. (2007), (c) Bao et al. (2011), and (d) Liu et al. (2013)..............................................................111

Figure (5.2- 2). Effect of gas on the power consumption of the anchor rotating in the coaxial mixing vessel at $N_{c}=180 \mathrm{rpm}$ and the speed ratios of 8,10 , and 12 .

Figure (5.2- 3). Comparison between the actual total gassed power of coaxial mixers and the predicted ones: (a) PBU-anchor and (b) PBD-anchor. .116

Figure (5.2- 4). Central impeller power contribution to the total gassed power of the aerated coaxial mixer at different speed ratios: (a) PBU-anchor, (b) PBD-anchor. .118

Figure (5.2- 5). Gassed to ungassed power ratio of the PBU impeller used as the central impeller for the coaxial mixer in the agitation of corn syrup solutions with a low viscosity (0.05 Pa.s) and a high viscosity (1.4 Pa.s) .118

Figure (5.2- 6). Effect of the speed ratio on the gassed power uptake of the anchor used in a PBUanchor coaxial mixer rotated in the corn syrup solutions with the viscosities of (a) $\mu=0.05 \mathrm{~Pa}$.s and (b) $\mu=1.40 \mathrm{~Pa} . \mathrm{s}$ .119

Figure (5.2- 7). Gassed power curve for the PBU-anchor coaxial mixer at different speed ratios and $\mu=0.05$ Pa.s: (a) $N_{c}=150 \mathrm{rpm}$ and (b) $N_{C}=180 \mathrm{rpm}$. .121 
Figure (5.2- 8). Gassed power curve for the PBD-anchor coaxial mixer at different speed ratios and $\mu=0.05$ Pa.s: (a) $N_{C}=150 \mathrm{rpm}$ and (b) $N_{C}=180 \mathrm{rpm}$.

Figure (5.2- 9). Gassed power curve for the PBU-anchor at different speed ratios, $N_{c}=180 \mathrm{rpm}$, and $\mu=1.4$ Pa.s

Figure (5.2- 10). Gassed power curve for the A310-anchor coaxial mixer rotated in water at different speed ratios and $N_{c}=150 \mathrm{rpm}$.

Figure (5.2- 11). Radial gas distribution attained by the PBU-anchor coaxial mixer at different speed ratios and $\mu=1.4$ Pa.s: (a) $N_{R}=18$, (b) $N_{R}=14$, (c) $N_{R}=10$, (d) $N_{R}=9$, and (e) $N_{R}=8$.

Figure (5.3- 1). Contribution of each class of bubble to the overall gas holdup measured using the tomography data obtained from (a) plane 2 and (b) plane 4.

Figure (5.3- 2). Local gas holdup inside the aerated coaxial mixing vessel at the central impeller speed of $150 \mathrm{rpm}$ and the gas velocity of $0.15 \mathrm{~m} / \mathrm{s}$, and different speed ratios: (a) $N_{R}=14$, (b) $N_{R}=12$, (c) $N_{R}=10$, (d) $N_{R}=8$, and (e) $N_{R}=5$.

Figure (5.3- 3). Overall gas holdup as a function of gas velocity and speed ratio.

Figure (5.3- 4). Comparison between the experimental gas holdup values and the predicted ones.

Figure (5.3- 5). Effect of the speed ratio on the Sauter bubble diameters captured at (a) plane 2 and (b) plane 4.

Figure (5.3- 6). Relation between the Sauter bubble diameter and the speed ratio at the central impeller height.

Figure (5.4- 1). Gas holdup values in an aerated coaxial mixer as a function of the speed ratio and fluid viscosity. 
Figure (5.4- 2). Contour plots of gas dispersion throughout the coaxial mixing system at different speed ratios for: (a) $\mu=2$ Pa.s, and (b) $\mu=1.4$ Pa.s

Figure (5.4- 3). Local gas holdup values at different speed ratios ( $\mu=2$ Pa.s).

Figure (5.4- 4). Gas holdup distributions at different heights $(z=0.09,0.146,0.202,0.258$, and, $0.314 \mathrm{~m}$ ) of the aerated coaxial mixer and different speed ratios.

Figure (5.4- 5). Gas holdup at the different speed ratios in the co-rotating and counter-rotating modes.

Figure (5.4- 6). Contour plots of turbulent kinetic energy at: (a) counter-rotating $N_{R}=14$, (b) corotating $N_{R}=14$, (c) counter-rotating $N_{R}=12$, (d) co-rotating $N_{R}=12$, (e) counter-rotating $N_{R}=10$, (f) co-rotating $N_{R}=10,(\mathbf{g})$ counter-rotating $N_{R}=8$, (h) co-rotating $N_{R}=8 \ldots \ldots . .$. Error! Bookmark not defined.

Figure (5.4- 7). Local turbulent kinetic energy data at different heights $(Z=0.09,0.146,0.202$, 0.258 , and, $0.314 \mathrm{~m}$ ) for the co- and counter-rotating aerated coaxial mixer.

Figure (5.4- 8). Velocity vectors generated by the aerated coaxial mixer in the co-rotating mode: (a) $N_{R}=14$, (b) $N_{R}=12$, (c) $N_{R}=10$, (d) $N_{R}=8$ .156

Figure (5.5- 1). Sauter mean bubble diameters estimated through the simulation (bold) and those obtained from the experiment in different points inside of the vessel. 163

Figure (5.5- 2). Effect of speed ratio on the volume fractions of the bubbles with different sizes.

Figure (5.5- 3). Effect of the speed ratio on dispersion of bubbles with different diameters within the aerated coaxial mixer ( $\mu=0.05$ Pa.s, $N_{c}=200 \mathrm{rpm}, N_{R}=8,10,12,14, Q_{g}=0.0283 \mathrm{~m}^{3} / \mathrm{min}$ ). 


\section{Nomenclature}

\begin{tabular}{|c|c|c|}
\hline$B_{\text {breakage }}$ & Birth rate due to breakage & $\mathrm{Kgm}^{-3} \mathrm{~s}^{-1}$ \\
\hline$B_{\text {aggregatio n }}$ & Birth rate due to coalescence & $\mathrm{Kgm}^{-3} \mathrm{~s}^{-1}$ \\
\hline$C$ & Impeller clearance & $\mathrm{m}$ \\
\hline$C_{D}$ & Drag coefficient & - \\
\hline$C_{\varepsilon 1}$ & $k-\varepsilon$ model constant & - \\
\hline$C_{\varepsilon 2}$ & $k-\varepsilon$ model constant & - \\
\hline$C_{\mu}$ & $k-\varepsilon$ model constant & - \\
\hline$C_{\mu p}$ & $k-\varepsilon$ model constant & - \\
\hline$D$ & Impeller diameter & $\mathrm{m}$ \\
\hline$D_{\text {breakage }}$ & Death rate due to breakage & $\mathrm{Kgm}^{-3} \mathrm{~s}^{-1}$ \\
\hline$D_{\text {aggregation }}$ & Death rate due to coalescence & $\mathrm{Kgm}^{-3} \mathrm{~s}^{-1}$ \\
\hline$d_{b}$ & Bubble diameter & $\mathrm{m}$ \\
\hline$F$ & Force & $\mathrm{N}$ \\
\hline$F_{D}$ & Drag force & $\mathrm{N}$ \\
\hline$g$ & Gravitational acceleration & $\mathrm{ms}^{-2}$ \\
\hline$g\left(V^{\prime}\right)$ & Breakage rate & $\mathrm{m}^{3} \mathrm{~s}^{-1}$ \\
\hline$g\left(V, V^{\prime}\right)$ & Coalecsence rate & $\mathrm{m}^{3} \mathrm{~s}^{-1}$ \\
\hline$H$ & Liquid height & $\mathrm{m}$ \\
\hline$k$ & Consistency index & $\operatorname{Pa~s}^{\mathrm{n}}$ \\
\hline$k$ & Turbulent kinetic energy & $\mathrm{m}^{2} \mathrm{~s}^{-2}$ \\
\hline
\end{tabular}




\begin{tabular}{|c|c|c|}
\hline$K_{p}$ & Impeller power constant & - \\
\hline$K_{s}$ & Metzner Otto constant & - \\
\hline$l$ & length & $\mathrm{m}$ \\
\hline$M$ & Torque & $\mathrm{Nm}$ \\
\hline$N$ & Impeller rotational speed & $\mathrm{rpm}$ or $\mathrm{s}^{-1}$ \\
\hline$N_{R}$ & Speed ratio & - \\
\hline$n$ & Shear thinning index & - \\
\hline$n(V, t)$ & Number Density of bubbles & $\mathrm{m}^{-3}$ \\
\hline$P_{o}$ & Ungassed power consumption & $\mathrm{W}$ \\
\hline$P_{g}$ & Gassed power consumption & $\mathrm{W}$ \\
\hline$Q$ & Gas flow rate & $\mathrm{m}^{3} \mathrm{~s}$ \\
\hline$T$ & Tank diameter & $\mathrm{m}$ \\
\hline$t$ & Time & $\mathrm{s}$ \\
\hline$t_{m}$ & Mixing time & $\mathrm{s}$ \\
\hline$u$ & velocity & $\mathrm{ms}^{-1}$ \\
\hline$V$ & Volume & $\mathrm{m}^{3}$ \\
\hline$X_{1}$ & Coded value of central impeller speed & $\mathrm{s}^{-1}$ \\
\hline$X_{2}$ & Coded value of anchor impeller speed & $\mathrm{s}^{-1}$ \\
\hline$X_{3}$ & Coded value of gas flow rate & $\mathrm{s}^{-1}$ \\
\hline$X_{4}$ & Coded value of viscosity & $\mathrm{s}^{-1}$ \\
\hline
\end{tabular}


Dimensionless numbers:

$\begin{array}{cc}\mathrm{Re} & \text { Reynolds number } \\ N_{p} & \text { Power number } \\ N_{\theta} & \text { Dimensionless mixing time } \\ \mathrm{Fr} & \text { Froude number } \\ \mathrm{Fl} & \text { Gas flow number } \\ E_{0} & \text { Eotvos number }\end{array}$

\section{Greek letters:}

\begin{tabular}{|c|c|c|}
\hline$\phi_{g}$ & Gas holdup & - \\
\hline$\varepsilon$ & Turbulent kinetic energy & $\mathrm{m}^{2} \mathrm{~s}^{-3}$ \\
\hline$\phi$ & Volume fraction & - \\
\hline$\gamma^{0}$ & Shear rate & $\mathrm{s}^{-1}$ \\
\hline$\gamma_{b}{ }^{0}$ & Gassed shear rate & $\mathrm{s}^{-1}$ \\
\hline$\eta$ & Effective viscosity & $\mathrm{Pa} \mathrm{s}$ \\
\hline$\mu$ & viscosity & Pa s \\
\hline$\rho$ & Density & $\mathrm{Kgm}^{3}$ \\
\hline$\tau$ & Shear stress & $\mathrm{Pa}$ \\
\hline$\tau_{y}$ & Yield stress & $\mathrm{Pa}$ \\
\hline$\sigma_{\varepsilon}$ & $k-\varepsilon$ model constant & - \\
\hline$\sigma_{k}$ & $k-\varepsilon$ model constant & - \\
\hline$\sigma$ & Surface tension & $\mathrm{Nm}^{-1}$ \\
\hline
\end{tabular}


Indexes:

l

$g$

$T$

$i$

$a$

c

$S$

\section{Abbreviations}

$P B U$

$P B D$

$R T$

SC

TXU

ERT

$C F D$

PBM

DGD

$S D$
Liquid phase

Gas phase

Turbulent

Liquid or gas phase

Anchor

Central impeller

Sparger

Pitched blade upward pumping

Pitched blade upward pumping

Rushton Turbine

SCABA impeller

Techmix335 Hydrofoil impeller

Electrical Resistance Tomography

Computational Fluid Dynamics

Population Balance Model

Dynamic Gas Disengagement

Standard deviation 


\section{Chapter 1}

\section{Introduction}

Mechanically stirred vessels are indispensable parts of variety of process industries such as chemical and biochemical processes, pharmaceutical, food industries, waste water treatment, and so on for single phase or multiphase flow agitation (Labik et al., 2014; Abdullah et al., 2011; Khopkar and Tanguy, 2008; Khopkar et al., 2007; Murthy et al. 2007). Two phase gas-liquid systems have attracted considerable attentions in many technologies especially biochemical engineering. The reason is attributed to the utilization of a gas phase in many fermentation processes. In such complex multiphase systems, agitation must provide not only the highest mass transfer rate, which is related to the gas holdup, but also the specified degree of homogeneity in the shortest possible time. Insufficient mixing in multiphase processes causes continuous variations in the surrounding environment of micro-organisms due to the formation of oxygen and nutrient segregation zones leading to the rapid strain degradation and a decreased process output (Espinosa-Solares et al., 2002; Vrabel et al., 2000; Lamberto et al., 1996). The accessibility and distribution of the nutrient throughout the fermenters are essential and can significantly alter the 
Chapter 1 Introduction

metabolic pathway and the biological product distribution. These segregation zones must be eliminated since they act as barriers to agitation processes and give rise to undesired by-products. Generally, an ideal agitation process must be successful in mixing the bulk fluid perfectly and simultaneously enhancing the gas-liquid contact.

Over the years, several approaches have been proposed to eliminate segregation regions (Pakzad et al., 2013a, 2013b, 2013c; Foucault et al., 2004, 2005, 2006; Espinosa-Solares et al., 2002; Lamberto et al., 1996). As an illustration, increasing the impeller rotational speed is employed in order to prevent the development of the aforementioned undesired zones. The basic drawback of this approach is excessive power uptake of the impeller especially in highly viscous fluids that causes mechanical damage. Using high rotational speed is also impractical in many biotechnological applications, where substances namely micro-organisms are shear-sensitive, and fast stirring leads to the reduction in productivity (Lamberto et al., 1996). It should be mentioned that when the shear sensitivity of the micro-organisms is defined as a significant factor in the design of the most efficient bioreactor, the multiple-impeller agitated tanks become more favorable. Multi-impeller aerated agitated tanks can be equipped with a number of different or identical impellers. These agitated tanks can provide higher surface per unit volume, more uniform distribution of the shear rate within the tank, and higher gas utilization rate (Moucha et al., 2009; Marko-poulos and Pantuflas, 2001). However, previous studies demonstrated that the traditional mixing systems with multiple impellers located on the same shaft still show some inefficiencies to disperse the gas evenly throughout the tank containing the viscous Newtonian and non-Newtonian fluids.

The coaxial mixers composed of a central impeller and a close clearance impeller have demonstrated highly efficient performances in the mixing of the single phase viscous fluids 
(Kazemzadeh et. al, 2016a, 2016b and 2017; Pakzad et al., 2013a, 2013b, and 2013c; Bonnot et al., 2007; Foucault et al., 2004, 2005). Since the central impeller and the close clearance impeller in a coaxial mixing system are attached to two different shafts, they can rotate at different speeds and directions separately, resulting in the enhancement of the homogenization throughout the vessel. In such a system, the main responsibility of the low speed anchor is to clean up the vessel wall and bringing back the bulk fluid, which has been accumulated away from the central impeller. The open impellers, which are usually located at the center of the coaxial mixer, operate at a high speed to generate vigorous shear. Although coaxial mixers have been extensively used in many applications including dispersion, emulsification, and viscous mixing; limited information is available in the literature regarding their applications in the mixing of the single-phase viscous Newtonian and non-Newtonian fluids. Most of the authors concentrated on the power consumption and mixing time of the aforementioned systems, particularly in the laminar and transitional regimes (Kazemzadeh et al., 2016a, 2016b, 2017; Pakzad et al., 2013a, 2013b, 2013c; Foucault et al., 2004, 2005, 2006; Thibault and Tanguy, 2002).

The coaxial mixers can be a desirable configuration to omit the oxygen and nutrient-starved regions in the aerated mixers containing highly viscous fluids (Newtonian/non-Newtonian). According to the author's knowledge no previous work has been conducted on the aerated coaxial mixers composed of a pitched blade turbine and an anchor. Therefore, the main objective of the current work is to investigate the performance of a novel aerated coaxial mixing vessel composed of a central impeller and a wall scraping anchor. To achieve this goal, the effects of various parameters including central impeller type, central impeller and anchor speeds, speed ratio, viscosity, and gas flow rate on the mixing time, power uptake by the impellers, gas hold-up, and 
Chapter 1 Introduction

bubble size distribution are assessed through the response surface methodology, advanced flow visualization technique (ERT), and computational fluid dynamics.

Chapter 2 provides a comprehensive literature review of the gas-liquid mixing systems equipped with different or identical impellers, and ungassed coaxial mixers. At the end of this chapter the research objectives are defined.

Chapter 3 describes the detailed experimental setup and procedures for the use of the tomography technique to measure mixing time and gas holdup. The dynamic gas disengagement technique coupled with tomography data for measuring the number of bubble classes, contribution of each class of bubble in the gas dispersion throughout the aerated coaxial mixer, and Sauter mean bubble diameter are explained. The methodology of conducting the experimental design and calculating the gassed power uptake are also included.

Chapter 4 describes the computational fluid dynamic model developed for the aerated coaxial mixing vessel. The governing equations for gas-liquid flow are presented. The CFD model is validated using the gas holdup and power uptake data. The grid independence test is performed as well. .

Chapter 5 discusses the experimental and CFD results. This chapter is divided into five subsections as:

Section 5.1 analyzes the mixing characteristics in an aerated coaxial mixer through electrical resistance tomography and response surface method.

Section 5.2 studies power consumption and gas holdup distribution for an aerated system equipped with a coaxial mixer. Also, novel correlations for the gas flow number and power number are proposed. 
Chapter 1 Introduction

Section 5.3 investigates the bubble behaviour in an aerated coaxial mixer using electrical resistance tomography, ERT.

Section 5.4 describes CFD simulation of hydrodynamic characteristics of the aerated coaxial mixing vessel equipped with the pitched blade turbine and the anchor.

Section 5.5 describes modelling of bubble size distributions within the coaxial mixer through the population balance coupled with CFD.

Finally, Chapter six summarises the main outcomes of this thesis and gives recommendations for the future works. 


\section{Chapter 2}

\section{Literature Review}

This chapter focuses on the review of the literature pertinent to the gas-liquid stirred vessels as well as coaxial mixing vessels in the absence of gas. Firstly, the dimensionless numbers, which can be used for analysing and presenting the data related to the stirred vessels, are described. Secondly, the conducted researches on the performances of the aerated mixing vessels in terms of power consumption, mixing time, gas holdup, and bubble size distribution are discussed. Finally, the modeling work carried out on the gas-liquid mixers are reviewed.

\subsection{Dimensionless numbers}

\subsubsection{Power number}

The power input is the amount of energy which is dissipated by the agitators throughout the fluid being mixed in the vessel. Power uptake in the stirred vessel is proportional to various parameters as (Rudolph, 2007):

$$
P=f(\rho, \eta, N, g, D, T, H, C \ldots)
$$


In addition to the aforementioned parameters: $D$ (impeller diameter), $T$ (tank diameter), $H$ (liquid height), $C$ (impeller bottom clearance) and so on; other variables also can affect the power consumption of the stirred vessel including number of impeller blades, number of baffles, and the distance between impellers in the multi-impeller configurations.

Using dimensionless analysis, number of dimensionless groups can be employed to express the power consumption in terms of power number $\left(N_{p}\right)$ as:

$$
N_{p}=\frac{P_{o}}{\rho N^{3} D^{5}}=C\left(\frac{\rho N D^{2}}{\mu}\right)^{a}\left(\frac{N^{2} D}{g}\right)^{b}\left(\frac{T}{D}\right)^{e}\left(\frac{H}{D}\right)^{d} \ldots
$$

here $P_{o}, N, \rho$, and $\mu$ are ungassed total power, impeller speed, fluid density, and fluid viscosity, respectively. Exponents are constants and are related to the specification of the given system. Power number is an important factor for comparing different configurations in the mixing process.

\subsubsection{Reynolds number}

Newtonian Reynolds number represents the ratio of inertial to viscous forces and can be used to identify the laminar, transitional or turbulent regimes.

$$
\operatorname{Re}=\frac{\rho N D^{2}}{\mu}
$$

The relation between Reynolds number and power number is dependent on the flow regimes. For instance, at low Reynolds number, laminar regime, $N_{p}$ is proportional to $\mathrm{Re}^{-1}$. Besides, at high Reynolds numbers, turbulent regime, $N_{p}$ is constant. The transitional regime occurs between the laminar and turbulent zones, at which no simple mathematical relationship exists between $N_{p}$ and 
Chapter 2: Literature Review

Re (Chhabra and Richardson, 1999). Therefore, by depicting the power curve (power number vs. logarithmic Reynolds number), in the laminar region power number is inversely proportional to Reynolds number $\left(N_{p} \approx \operatorname{Re}^{-1}\right)$, indicating that:

$$
N_{p}=K_{p} \times \operatorname{Re}^{-1}=K_{p} \times\left(\frac{\rho N D^{2}}{\mu}\right)^{-1}
$$

where $K_{p}$ depends on the agitator type and geometrical characteristics of the stirred vessel as summarized in the Table 2.1-1. The variations of the proposed values of the $K_{p}$ for a specific agitator type are related to the different characteristics of the employed mixing vessel.

Table (2.1- 1). $\boldsymbol{K}_{p}$ values for different impellers

\begin{tabular}{lll}
\hline Impeller Type & $K_{p}$ & Reference \\
\hline \multirow{3}{*}{ Anchor } & 194 & Novak and Rieger (1975) \\
& 203 & Takahashi et al., (1980) \\
& 420 & Thibault and Tanguy (2002) \\
\hline \multirow{2}{*}{ Double Helical Ribbon } & 296 & Zlokarnik (1988) \\
& 351 & Takahashi et al., (1980) \\
& 276.6 & Rieger et al., (1986) \\
\hline Rushton & 1000 & Zlokarnik (1988) \\
\hline 70 & Nagata et al., (1972) \\
\hline Pitched blade turbine & 75 & Mentzner and Otto (1957) \\
\hline
\end{tabular}


Therefore, when Newtonian fluids are involved in the process power number can be easily calculated by using $K_{p}$, agitator rotational speed, agitator diameter, fluid density, and fluid viscosity. While in the case of non-Newtonian fluids, the viscosity is not constant and there is a complex function between the shear rate produced by the agitator and the fluid viscosity. Hence, an appropriate viscosity based on the fluid rheology should be calculated and substituted in the Equation (2.1-4).

\subsubsection{Mixing with aeration}

In the presence of gas, there is an expectation of liquid deformation caused by bubbles rising in the liquid. Therefore, shear rate of liquid is defined as a combination of shear rates produced by bubbles and also created by the impeller as:

$$
\gamma^{\circ}=\sqrt{\gamma_{b}^{\circ 2}+\gamma_{e}^{\circ 2}}
$$

where $\gamma_{e}^{\circ}$ is the shear rate generated by impeller rotation in the liquid under gas-free conditions, which can be calculated from Metzner and Otto technique (Metzner and Otto, 1957). $\gamma_{b}^{\circ}$ is determined as an additional shear rate induced on the liquid due to the bubble motion and can be estimated by Hashikawa and coworkers correlation (Cheng and Carreau, 1994).

$$
\gamma_{b}^{\circ}=1500 u_{g}
$$

here $u_{g}$ is superficial gas velocity. Therefore, because of the additional term of shear rate, $\gamma_{b}$, aerated Reynolds number is higher than the unaerated one and it enhances by increasing the gas flow rate.

\subsubsection{Froude number}

Froude number is defined as the ratio of the inertial forces to the gravitational forces as: 
Chapter 2: Literature Review

$$
F r=\frac{N^{2} D}{g}
$$

Froude number is not suitable as a scale-up criterion and only is applicable where vortex exists (Rudolph, 2007). Also, the mentioned dimensionless number can be used to estimate the aerated impeller power uptake (Bakker et al., 1995). In the presence of gas, both Froude number and gas flow number can be employed to characterize the types of developed cavities and also onset of flooding, as described in details in section 2.2 (Middleton and Smith, 2004).

It is worth noting that Froude number can be neglected in a baffled agitated tank or in the unaerated vessel working at low Reynolds number because of the elimination of swirls. Accordingly, in such a situation the power number is only proportional to the Reynolds number.

\subsubsection{Gas flow number}

Gas flow number (aeration number) is denoted as the ratio of the gas volumetric flow rate to the impeller pumping capacity:

$$
F l_{g}=\frac{Q_{g}}{N D^{3}}
$$

where $Q_{g}$ is gassing rate from the sparger. Gas flow number which is important in determining the flow phenomena occurring in the impeller region includes the effects of gassing rate, impeller rotational speed, and impeller diameter. The aforementioned dimensionless number is constant under turbulent regimes (Nagata, 1972). 
Chapter 2: Literature Review

\subsection{Aerated flow pattern}

The gas-liquid flow pattern plays a crucial role in the aerated vessels. When the gas is distributed into the vessel, different flow patterns develop depending on the gas flow rate and impeller rotational speed. Flow patterns are categorized in three groups as: flooding (F), loading (L) and complete dispersion (CD). Transition between the aforementioned regimes can be observed by changing the operating parameters such as gas flow rate and impeller speed. Either at low impeller speed or high gas flow rate; flow pattern is dominated by gas flow which travels up through the middle of the vessel without being affected by the rotating impeller. In this case, impeller is flooded and gas dispersion is inefficient, as shown in Figure 2.2-1a. At higher impeller speeds or lower gas flow rates, the onset of gas dispersion happens and gas bubbles accumulated in the low pressure area behind the blades which is called loading, Figure 2.2-1b. Further increase in impeller speed or decline in gas flow rate lead to compete dispersion, Figure 2.2-1c, (John, 1998). At this condition, the impeller disperses the gas towards the tank walls and bubbles reach even the bottom of the tank. This regime with complete dispersion of bubbles throughout the aerated vessel is the desirable condition. 
Chapter 2: Literature Review

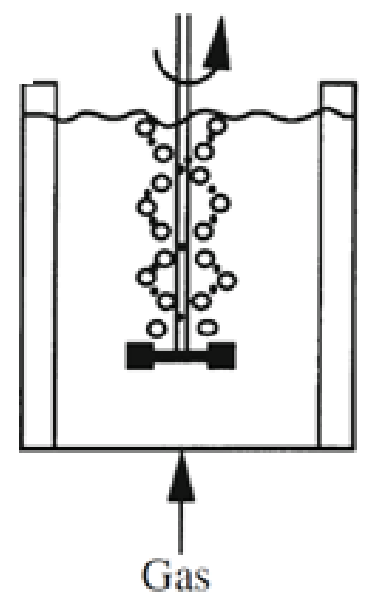

(a)

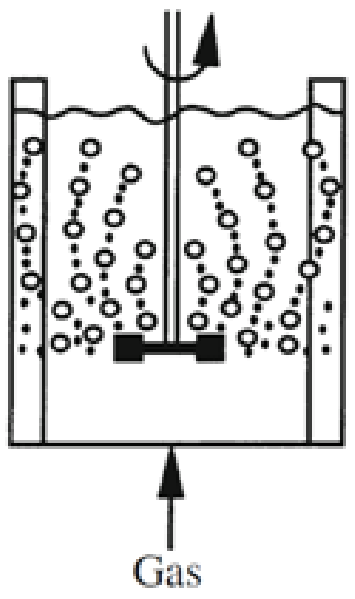

(b)

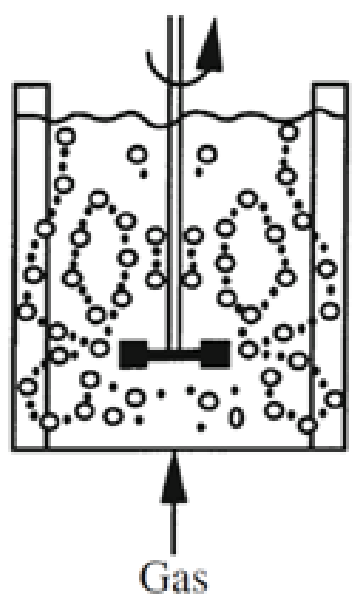

(c)

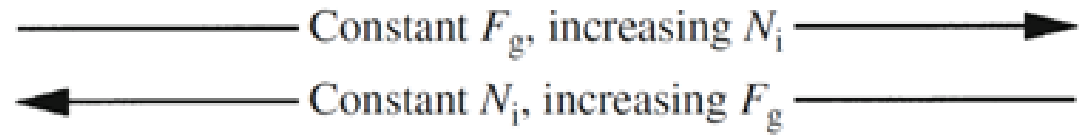

Figure (2.2- 1). Gas distribution patterns in the aerated vessel equipped with disc turbine as a function of impeller speed and gas flow rate (John, 1998).

It is worth noting that transition between the mentioned regimes can be estimated in terms of two dimensionless groups, Flow number and Froude number, as:

$$
\begin{gathered}
F l_{g}=\frac{Q_{g}}{N_{i} D_{i}^{3}} \\
F r=\frac{N_{i}^{2} D_{i}}{g}
\end{gathered}
$$

Several correlations have been proposed for estimating the flooding-loading and loading-complete dispersion transitions, as illustrated in Table 2.2-1. 
Table (2.2- 1). Proposed correlations for predicting the transition between different flow patterns developed in the gas-liquid mixing vessels.

\begin{tabular}{|c|c|c|c|}
\hline Description & Limitations & Correlation & Reference \\
\hline Flooding-loading transition & Disk turbine & $F l_{g}=30 F r(D / T)^{3.5}$ & Junker et al. (1998) \\
\hline Flooding-loading transition & 2 Rushton turbine & $\begin{array}{l}\text { Lower impeller } F l_{g}=0.25(F r)^{0.75} \\
\text { Upper impeller } F l_{g}=1.08(F r)\end{array}$ & Taghavi et al. (2011) \\
\hline $\begin{array}{l}\text { Loading-complete } \\
\text { dispersion }\end{array}$ & Rushton turbine & $F l_{g}=13(F r)^{2}(D / T)^{5}$ & Nienow et al. (1977) \\
\hline $\begin{array}{l}\text { Loading-complete } \\
\text { dispersion }\end{array}$ & Rushton turbine & $F l_{g}=0.2(D / T)^{0.5}(F r)^{0.5}$ & Nienow (1990) \\
\hline $\begin{array}{l}\text { Loading-complete } \\
\text { dispersion }\end{array}$ & Rushton turbine & $F l_{g}=1.7(F r)^{3}$ & Taghavi et al. (2011) \\
\hline
\end{tabular}

\subsection{Power consumption}

Power draw is the most important factor to characterize the mixing process. This variable is expressed as the amount of energy required in order to produce the movement of the fluids in the vessel through mechanical agitation. It should be mentioned that the costs associated with the power consumption contribute remarkably to the overall operation costs of the mixing process. So, in order to achieve a cost-effective operation, it is desirable to minimize the power draw of the agitators. Additionally, power consumption affects heat and mass transfer processes, mixing time, and circulation time. In the presence of gas, aerated power uptake can be characterized using different dimensionless groups including gas flow number, Weber number, and Froude number as discussed in the following section. 
Chapter 2: Literature Review

\subsubsection{Power requirement for gas-liquid stirred vessel}

Power uptake of impellers in the gas-free stirred tanks has been well developed in terms of both Newtonian and non-Newtonian fluids (Pakzad et al., 2008, 2013b 2013c; Foucault et al., 2004, 2005, 2006; Rushton et al., 1950). In the absence of gas, power requirement of the mixing vessel can be estimated from correlations of power number and Reynolds number (Rushton et al., 1950). In the gassed conditions, power dissipated from the impeller into the liquid is affected and dropped by aeration. The explanation lies on the bulk flow patterns as well as structure of the cavities generated behind the impeller blades (Wang et al. 2006). When gas is sparged into the vessel, distribution of the liquid by gas causes formation of the low pressure regions behind the blades; at which bubbles can easily accumulate and generate gas filled cavities. These cavities affect the effectiveness of the gas dispersion in the agitated vessel.

In general, power consumption of the aerated mechanically agitated vessel depends on the impeller type, impeller rotational speed, and gas flow rate. Traditional gas-liquid stirred vessel employed radial impellers such as Rushton turbine for gas dispersion. Several studies have shown that in the aerated condition, the reduction in power consumption of the Rushton impeller is about $50 \%$ (Nienow and Bujalski, 2004). Additionally, occurrence of compartmentalization and axial flow barriers are associated with Rushton turbine (Cronin et al., 1994). Therefore, with the aim of increasing the performance of gas-liquid mixing vessels, an increased attention to axial impellers has been evolved. Axial flow impellers provide considerable advantages over radial impellers in the presence of gas (Machon \& Jahoda, 2000; Vrabel et al., 2000; Nienow, 1998; McFarlane \& Nienow, 1996; Otomo et al., 1995; Cronin et al., 1994; Cooke et al., 1988). Axial flow impellers can operate either in downward or upward modes. The main drawback of downward pumping axial impeller is that they 
Chapter 2: Literature Review

subject to torque and flow fluctuations in the presence of gas (Nienow, 1998). Accordingly, axial upward pumping impellers appeared to be promising in the gassed condition. Employing upward pumping axial impellers in gas-liquid agitated vessels leads to a reduction in torque and flow instabilities. Furthermore, these types of impellers show better gas dispersion characteristics, loose little power in comparison with the radial impellers upon gassing, and handle more quantities of gas without flooding.

In regards to the effect of aeration on the power consumption of multiple impeller agitated vessels; several works have been reported (Taghavi, 2011; Cui et al., 1996; Abrardi et al., 1990; Hicks and Gates, 1976). The obtained results indicated that the reduction in the power drawn by the agitator located close to the sparger in much more considerable than that for the other impellers. Since most of the freshly sparged gas will go through the bottom agitator and only a fraction of that reach to the upper impellers. Therefore, bottom impeller acts as single impeller aerated mixing vessels.

In the open literature different correlations have been proposed for predicting the gassed to ungassed power ratio $\left(p_{g} / p\right)$, and aerated power uptake by the impellers $\left(p_{g}\right)$ as summarized in Table 2.3-1.

Table (2.3- 1). Several correlations for estimating power consumption in the presence of gas.

\begin{tabular}{llll}
\hline Impeller & Limitations & Correlation & Reference \\
\hline
\end{tabular}


Chapter 2: Literature Review

\begin{tabular}{|c|c|c|c|}
\hline $\begin{array}{l}\text { Disk } \\
\text { turbine }\end{array}$ & Water & $p_{g}=0.812\left(\frac{p_{O}^{2} N D^{3}}{Q_{g}^{0.56}}\right)^{0.45}$ & Michel and Miller (1962) \\
\hline $\begin{array}{l}\text { Six blade } \\
\text { turbine }\end{array}$ & $\begin{array}{c}\text { Water } \\
\text { Ethylene glycol }\end{array}$ & $P_{g} / P_{o}=0.497\left(\frac{Q_{g}}{N D^{3}}\right)^{-0.38}\left(\frac{N^{2} D^{3} \rho_{l}}{\sigma}\right)^{-0.18}$ & $\begin{array}{l}\text { Luong and Volesky } \\
\qquad(1979)\end{array}$ \\
\hline $\begin{array}{l}\text { Six blade } \\
\text { turbine }\end{array}$ & $\mathrm{CMC}$ & $P_{g} / P_{o}=0.514\left(\frac{Q_{g}}{N D^{3}}\right)^{-0.38}\left(\frac{N^{2} D^{3} \rho_{l}}{\sigma}\right)^{-0.194}$ & $\begin{array}{l}\text { Luong and Volesky } \\
\qquad(1979)\end{array}$ \\
\hline $\begin{array}{l}\text { Flat blade } \\
\text { turbine }\end{array}$ & Water & $P_{g} / P_{o}=0.1\left(\frac{Q}{N V}\right)^{-1 / 4}\left(\frac{N^{2} D^{4}}{g w V^{2 / 3}}\right)^{-1 / 5}$ & $\begin{array}{l}\text { Hughrnark } \\
\text { (1980) }\end{array}$ \\
\hline $\begin{array}{l}\text { Pitched blade } \\
\text { turbine }\end{array}$ & Water & $p_{g}=1.52\left(\frac{P_{O}^{2} N D^{3}}{Q_{g}^{0.56}}\right)^{0.427}$ & $\begin{array}{l}\text { Shewale and Pandit } \\
\text { (2006) }\end{array}$ \\
\hline
\end{tabular}

\subsubsection{Coaxial Power requirement in the absence of air}

Coaxial mixers are promising approach to eliminate flow compartmentalization and segregation. Although coaxial mixing vessels are employed in the wide range of industrial applications; the data available regarding the coaxial mixing is still poor and inadequate and their design and operation remain often empirical. Several studies have been conducted on the power consumption of coaxial configurations with Newtonian and non-Newtonian fluids (Kazemzadeh 2016a, 2016b, 2017; Pakzad et al., 2013a; Rivera et al., 2009; Rudolph et al., 2007; Bonnet et al., 2007; Foucault et al., 2004, 2005, 2006; Kohler and Hemmerle, 2003; Thibault and Tanguy, 2002). Because of the complexities associated with coaxial agitators (several types of impellers, different agitation speeds, and two rotation modes), it is difficult to characterize the performance of such a system in terms of power uptake. All of the conducted works demonstrated that total power consumption of the unaerated coaxial vessel can be affected by several factors including speed ratio, rotation modes, and system 
Chapter 2: Literature Review

geometry. Table 2.3-2 lists various correlations proposed based on generalized Reynolds number and power number to have a master power curve for coaxial mixers.

Table (2.3- 2). Different correlations offered by various authors for Reynolds number and power number of coaxial mixers.

\begin{tabular}{|c|c|c|}
\hline Recommended Correlations & Fluids & Reference \\
\hline $\begin{array}{l}N_{p}=\frac{P_{t o t}}{\rho N_{a}^{3} D_{a}^{5}} \\
\operatorname{Re}_{u g}=\frac{N_{a}^{2-n} D_{a}^{2} \rho}{K K_{R_{N}} K_{S\left(R_{N}\right)}^{n-1}}\end{array}$ & power-law & $\begin{array}{l}\text { Thibault and Tanguy } \\
\text { (2002) }\end{array}$ \\
\hline $\begin{array}{l}N_{p}=\frac{P_{t o t}}{\rho\left(N_{c} \pm N_{a}\right)^{3} D_{a}^{5}} \\
\operatorname{Re}_{u g}=\frac{\left(N_{c} \pm N_{a}\right)^{2-n} D_{C}^{2} \rho}{K K_{S(C I)}^{n-1}}\end{array}$ & Power-law & $\begin{array}{l}\text { Foucault et al. } \\
(2005,2006)\end{array}$ \\
\hline $\begin{array}{l}N_{p}=\frac{P_{t o t}}{\rho\left(N_{c} D_{c}+N_{a} D_{a}\right)^{2} D_{c}^{5}} \\
\operatorname{Re}_{u g}=\frac{\left(N_{c} D_{c}+N_{a} D_{a}\right) D_{c} \rho}{\mu}\end{array}$ & Power-law & $\begin{array}{l}\text { Farhat et al. } \\
\qquad(2007)\end{array}$ \\
\hline $\begin{array}{l}N_{p}=\frac{P_{t o t}}{\rho N_{a}^{3} D_{a}^{5}} \\
\operatorname{Re}_{u g}=\frac{N_{a}^{2-n} D_{a}^{2} \rho}{K K_{t r} K_{S(t o t)}^{n-1}}\end{array}$ & Power-law & $\begin{array}{l}\text { Rudolph et al. } \\
\qquad \text { (2007) }\end{array}$ \\
\hline $\begin{array}{l}N_{p}=\frac{P_{t o t}}{\rho\left(N_{c}+f_{p(a)} N_{a}\right)^{3} D_{a}^{5}} \\
\operatorname{Re}_{u g}=\frac{K_{S(c)}\left(N_{c}+f_{p(a)} N_{a}\right)^{2} D_{c}^{2} \rho}{\tau_{y}+K\left(K_{S(c)}\right)^{n}\left(N_{c}+f_{p(a)} N_{a}\right)^{n}}\end{array}$ & Herschel-Bulkley & $\begin{array}{l}\text { Pakzad et al. } \\
\text { (2013d) }\end{array}$ \\
\hline
\end{tabular}




\subsection{Gas holdup}

In the many of industrial processes mechanically agitated vessels are utilized in order to carry out reactions between gas and liquid. At which, the quality of the gas dispersion and effectiveness of the gas-liquid mass transfer which are the main functions of the impeller can be characterized by one of the key parameters called gas holdup. Gas holdup is expressed as the ratio of the gas phase volume to the total volume as follows:

$$
\phi_{g}=\frac{V_{G}}{V_{G}+V_{L}}
$$

$V_{G}$ and $V_{L}$ are gas and liquid volumes, respectively.

\subsubsection{Gas holdup in single and multi-impeller configurations}

Mixing vessels equipped with single impeller are employed when height to diameter ratio is not large. Generally, multiple impellers are preferred over single impeller ones. For instance, at the equivalent power input and gas sparging rate, multi-impeller configurations have been found ensuring higher gas holdup values than single impeller ones (Bouaifi and Roustan, 2001; Arjunwadkar et al., 1998).

It is worth noting that increasing the gas holdup values are proportional to the number of impellers. It can be observed that the effect of increasing the number of impellers from 1 to 2 on the fractional gas holdup is more considerable than that from 2 to 3 . The influence of the number of impellers on the fractional gas holdup can be given as follows: 
Chapter 2: Literature Review

$$
\phi_{g} \times n_{i}^{x}
$$

here $n_{i}$ is specified as the number of stirrers and $\phi_{g}$ is the fractional gas holdup. Exponent $x$ is related to the number of impellers. By increasing the number of impellers from 1 to 2 , the exponent $x$ is equal to 1 ; however, the value of $x$ is in the range of 0.3-0.4 when $n_{i}$ is altered from 2 to 3 (Gogate et al., 2000).

\subsubsection{Effect of liquid viscosity on the gas holdup}

The effects of viscosity on the fractional gas holdup have been rarely reported in the literature (De Jesus et al., 2017; Ranade and Deshpande, 1999; Nienow and Ulbrecht 1985). Deformation of the bubbles is related to liquid viscosity. The higher the viscosity, the more the bubbles are deformable. Therefore, coalescence happens easier and faster (Zahradnik et al., 2001) and bubble breakup is ceased ( $\mathrm{Li}$ and Prakash, 2000). The reason is that higher viscosities have a tendency to reduce turbulence and therefore have negative effect on the bubble breakup. At which, because of the enhanced size and buoyancy, large bubbles tend to rise toward the surface level rapidly and hence their residence time is reduced, resulting in lower gas holdup. Arjunwadkar et al. (1998) reported about $30 \%$ reduction in the actual fractional gas holdup values for the viscous liquids in comparison with the air-water system.

\subsubsection{Proposed correlations for measuring gas holdup}

Fractional gas holdup is specific to the input energy and gas sparging rate which can be estimated by the following empirical correlation. 
Chapter 2: Literature Review

$$
\phi_{g}=c\left(\frac{P_{o}}{V}\right)^{a}\left(u_{g}\right)^{b}
$$

where $a, b$ and $c$ are constants. There are some controversies related to the aforementioned constants. Several workers proposed that these constants depend on the impeller type and their combinations, impeller bottom clearance, D/T ratio, and impeller diameter (Moucha et al., 2003). However, the others reported that the values of the mentioned constants were independent of the impeller type and only was a function of the fluid being used (Bujalski et al., 2002). Some of the proposed correlations for estimation the gas holdup are summarized in Table 2.4-1.

Table (2.4- 1). Correlations for gas holdup estimation. 


\begin{tabular}{|c|c|c|c|c|}
\hline Impeller Type & Operating condition & Solution & Equation & Reference \\
\hline RT & $\begin{array}{c}Q_{g}=2.12,4.248 .48 \mathrm{~mm} / \mathrm{s} \\
N=250-850 \mathrm{rpm}\end{array}$ & $0.5 \mathrm{M} \mathrm{Na}_{2} \mathrm{SO}_{4}$ & $0.01686\left(\frac{P}{V_{L}}\right)^{0.6241} u_{g} 0.5669$ & Moucha et al., (2003) \\
\hline 2RT & $\begin{array}{c}Q_{g}=2.12,4.248 .48 \mathrm{~mm} / \mathrm{s} \\
N=250-850 \mathrm{rpm}\end{array}$ & $0.5 \mathrm{M} \mathrm{Na}_{2} \mathrm{SO}_{4}$ & $0.05051\left(\frac{P}{V_{L}}\right)^{0.4903} u_{g} 0.5788$ & Moucha et al., (2003) \\
\hline PBD & $\begin{array}{c}Q_{g}=2.12,4.248 .48 \mathrm{~mm} / \mathrm{s} \\
N=250-850 \mathrm{rpm}\end{array}$ & $0.5 \mathrm{M} \mathrm{Na}_{2} \mathrm{SO}_{4}$ & $0.04656\left(\frac{P}{V_{L}}\right)^{0.4666} u_{g} 0.5816$ & Moucha et al., (2003) \\
\hline 2PBD & $\begin{array}{c}Q_{g}=2.12,4.248 .48 \mathrm{~mm} / \mathrm{s} \\
N=250-850 \mathrm{rpm}\end{array}$ & $0.5 \mathrm{M} \mathrm{Na}_{2} \mathrm{SO}_{4}$ & $0.08193\left(\frac{P}{V_{L}}\right)^{0.5202} u_{g} 0.7064$ & Moucha et al., (2003) \\
\hline RT+ PBD & $\begin{array}{c}Q_{g}=0.29-0.975 v v m \\
N=400-750 \mathrm{rpm}\end{array}$ & $\mathrm{CMC}$ & $0.064\left(\frac{P_{g}}{V}\right)\left(1-\phi_{g}\right)^{0.4} u_{g}^{0.5}$ & $\begin{array}{c}\text { Arjunwadkar et al., } \\
\text { (1998) }\end{array}$ \\
\hline $\mathbf{R T}+\mathbf{T X U}$ & $\begin{array}{c}Q_{g}=2.12,4.248 .48 \mathrm{~mm} / \mathrm{s} \\
N=250-850 \mathrm{rpm}\end{array}$ & $0.5 \mathrm{M} \mathrm{Na}_{2} \mathrm{SO}_{4}$ & $0.03110\left(\frac{P}{V_{L}}\right)^{0.5071} u_{g} 0.5005$ & Moucha et al., (2003) \\
\hline $\mathbf{R T}+\mathbf{P B D}$ & $\begin{array}{c}Q_{g}=2.12,4.248 .48 \mathrm{~mm} / \mathrm{s} \\
N=250-850 \mathrm{rpm}\end{array}$ & $0.5 \mathrm{M} \mathrm{Na}_{2} \mathrm{SO}_{4}$ & $0.04668\left(\frac{P}{V_{L}}\right)^{0.4970} u_{g} 0.5718$ & Moucha et al., (2003) \\
\hline
\end{tabular}

\subsubsection{Gas holdup measuring techniques}

Gas holdup measurement techniques can be broadly classified into two different ways; globally or locally. Global gas holdup measurement has been conducted by observing the difference between liquid height under gassed and ungassed conditions either visually or from pressure differences (Yawalkar et al., 2002; Arjunwadkar et al., 1998; Rushton and Bimbinet, 1968). The measurement of global gas holdup which provides information about the average gas holdup is relatively simple. Nonetheless, local gas holdup measurement is extremely a difficult task and the knowledge of that is 


\section{Chapter 2: Literature Review}

required for validating the numerical results and also for trustworthy design. Calderbank (1958) was the first author who addressed the problems associated with the local gas holdup measurement. Local gas holdup measurement techniques can be categorized into invasive and non-invasive groups. Some of the invasive techniques have been reported in the literature including heat transfer probe (Boyer et al., 2002), needle probe (Wang et al., 2006; Kumar et al., 1995), and suction methods (Nagase and Yasui, 1983; Nienow et al., 1977). The problem associated with the invasive methods is that they yield the disturbance of the flow inside the tank. The mentioned limitations regarding the invasive techniques can be overcome by adopting non-intrusive approaches. With respect to non-intrusive characteristics, several methods have been reported such as ultrasonic (Supardan et al., 2004; Utomo

et al., 2001; Chaouki et al., 1997; Fischer et al., 1992), electrical capacitance tomography (Warsito and Fan 2001; Chaouki et al., 1997; Halow, 1995), electrical resistance tomography (ERT) (Kazemzadeh et al., 2017; Wang et al., 2000; Chaouki et al., 1997) , and $X$ ray and $\gamma$ ray (Ford et al., 2008; Khopkar et al., 2005; Thatte et al., 2004). By comparing the suggested invasive and noninvasive measuring techniques and also by considering their limitations and drawbacks, the noninvasive electrical resistance tomography (ERT) has been found to be a superior technique to perform the flow visualization and to obtain the local gas holdup values due to its low cost and simplicity (Scott and McCann, 2005) and also in contrast to the radiation based tomography methods; it is safe for the experimenter (Mann et al., 1997a, 1997b). Using this technique, the 3D concentration field inside the vessel can be verified and the transition in the flow can be tracked rapidly (Mann et al., 1999). ERT has been used successfully for acquiring radial and axial distribution of gas holdup in bubble columns (Babaei et al., 2015a, 2015b; Fransolet et al., 2001). In this approach the differences 
Chapter 2: Literature Review

in the conductivities of the involved phases are used to map the phase distribution. In the gas-liquid stirred vessels gas is considered as the nonconductive phase, while liquid is the conductive phase.

\subsection{Mixing Time}

Homogeneity which is described in terms of mixing time is the most important parameter in the efficient design of the mixing process. Mixing time is assigned as the required time to obtain a predefined level of homogeneity within the vessel. The aforementioned factor is related to a number of parameters including fluid properties, impeller speeds, impeller types, impeller and vessel geometries, number of impellers, and gas flow rates. The shorter the blending time the more effective the mixing.

\subsubsection{Single impellers}

A number of works have been documented on the mixing times of configurations stirred by single impellers as reviewed by Nere et al. (2003) and Grenville and Nienow (2004), comparatively.

It is usually desirable to compare the level of homogeneity of different mixer configurations. Therefore, the dimensionless mixing time, $N \theta_{95}$, is defined to characterize the blending time. The aforementioned time is the time at which $95 \%$ of homogeneity is achieved. It depends to a large extent on the vessel geometry, operating conditions, and fluid properties as follows:

$$
f\left(N \theta_{95}, N, \rho, \eta, D, T, W, H \ldots\right)=0
$$

By applying the rules of the dimensionless analysis, the majority of works correlated mixing times by using dimensionless groups including Reynolds number, Froude number, and total specific power input into the system or energy dissipation rate. Among them, most of the workers have demonstrated that under turbulent conditions dimensionless mixing time, $N \theta$, is constant and independent of $\operatorname{Re}$ or 
Chapter 2: Literature Review

Fr numbers (Shaw, 1994; Abradi et al., 1990; Cook et al., 1988; Shuie and Wong, 1984). Rielly and Britter (1985) showed the dependency of the mixing time on $R e$ number under laminar regime. Fradette et al. (2007) indicated that mixing time was a function of Reynolds number and varied from one regime to another. Furthermore, Brennan and Lehrer (1976) found $N_{\theta} \propto \mathrm{Fr}^{a}$. Some of the authors proposed that dimensionless mixing time was inversely proportional to the impeller speeds (Gogate et al., 2000). Additionally, influence of the impeller types on the mixing time have been surveyed in several researches (Langheinrich, et al., 1995; Grenville 1995; Shaw, 1994; Hass and Nienow, 1989). They showed that mixing time was related to the total power input of the impeller not the impeller types. However, Verabel et al. (2000) expressed that up-pumping hydrofoil impellers can reduce mixing time by more than $50 \%$ in comparison with the radial ones under the same operating conditions.

\subsubsection{Dual impellers}

Technological advantages cause industry to use multi-impeller configurations instead of single impeller ones for mixing processes; especially gas-liquid systems. Various combinations of impellers including radial-radial, radial -axial, axial-axial, radial-close clearance impeller, and axial-close clearance impeller can be utilized in dual-impeller configurations. The reason is addressed to the better heat transfer, longer gas bubble residence time as well as occupying less space in a plant. Generally, dual-impeller has been utilized in a system where its height-to-diameter ratio exceeds 1.0 (Wang et al., 2000). Cronin et al., (1994) reported that at aspect ratio greater than one, 2:1, dual agitator vessels exhibit better mixing efficiency than single impeller ones at similar energy input.

Considering aforementioned configurations, flow pattern developed by one impeller can be affected by the other impeller in the vessel. The reason is attributed to the flow interactions between 
the agitators. Therefore, mixing time may change in such configurations. Several authors reported that incorporation of axial flow impellers rather than radial-radial ones, with equivalent power uptake, can halve the mixing time (Verabel et al., 2000; Abardi et al., 1990).

It is worth mentioning that most of the researches have been typically focused on the configurations equipped with dual impellers on the same shaft. A less number of studies have been carried out on the independently-driven dual impellers. Espinosa-Solares et al. (2002) proposed that combination of a close clearance and open impellers such as helical ribbon and Rushton turbine could reduce the mixing time remarkably. Other alternative novel combinations with two separately stirred impellers are coaxial mixers. Only few research papers have been published on the mixing time of Newtonian and non-Newtonian fluids in coaxial mixers which are equipped with a central impeller and a wall scraping anchor being stirred in co-rotating or counter-rotating modes (Pakzad et al., 2013a, 2013b; Bao et al., 2011; Rudolph et al., 2007; Foucault et al., 2004, 2005, 2006). Most of the works demonstrated that the performance of the coaxial systems operating in co-rotating mode with Newtonian and non-Newtonian fluids exhibit higher performance in comparison to the ones in counter-rotating mode and single impeller systems (Pakzad et al., 2013a, 2013b; Bao et al., 2011;Rudolph et al., 2007; Foucault et al., 2006). However, no data have been reported on the mixing time of the aerated coaxial systems due to the complications arising from complexity of the hydrodynamics and use of different types of agitators and criteria.

\subsubsection{Aerated mixing time}

Much less research has been carried out to define the effects of aeration on the mixing time of mechanically agitated vessels and it is still an open question. Considering the single impeller vessel, some studies demonstrated that aeration decreases the mixing time (Vasconcelos et al., 1995), while 
others (Hadjiev et al., 2006; Machon \& Jahoda, 2000) indicated that mixing time can be enhanced and reduced by gassing, according to the dominance of gas flow rate or impeller rotational speed. For example, Blakebrough and Sambamurthy (1966) reported that mixing time was enhanced by aeration. Using a configuration equipped with dual axial and mixed impellers, Abardi et al. (1990) observed that blending time was reduced by increasing the gas flow rate at low impeller speed. To be illustrated, at the lower impeller speed gas flow is dominated, flooded impeller, and stirred vessel acts as a bubble column. Therefore, the influence of the agitator on the liquid circulation can be neglected, leading to a reduction in the blending time. Correspondingly, at complete dispersion, mixing is controlled by the impeller action and increasing the gas flow rate decreases the mixing time. Bouaifi and Rouston (2001) expressed that at complete dispersion regime, mixing time remains constant and it is not proportional to the gas flow rate, vessel configuration and impeller diameters.

Besides, several studies were conducted to investigate the effect of impeller speed and superficial gas velocity on the mixing time of gas-liquid contactors agitated by multiple impellers (Patwardhan and Gaikwad, 2003; Machon and Jahoda, 2000; Otomo, 1995; Nienow and Elson, 1988). The impact of various factors including impeller speed, gas flow rate, spacing between impellers, and flow pattern were discussed in details in the literatures (Bouaifi and Roustan, 2001; Zhao et al., 2001). It was concluded that effect of aeration on the mixing time is dependent on the operating regime induced by the impellers, gas, or combination of both.

Some of the correlations that relate the mixing time to the geometrical and operating conditions of the agitated vessel are explained in Table 2.5-1.

Table (2.5- 1). Mixing time correlations for single and multi-impeller systems. 
Chapter 2: Literature Review

\begin{tabular}{llc}
\hline Configuration & correlation & Reference \\
\hline 6RT & $N \theta=20.41\left[\frac{a H+T}{T}\right]\left(\frac{T}{D}\right)^{13 / 6}\left(\frac{W}{D}\right)\left(\frac{Q_{g}}{N V}\right)^{1 / 12}\left(\frac{N^{2} D^{4}}{g W V^{2 / 3}}\right){ }^{1 / 15}$ & Pandit et al., 1983 \\
RT & $N \theta=5.3\left(\frac{1}{N}\right)\left(\frac{1}{P_{O}^{1 / 3}}\right)\left(\frac{D}{T}\right)^{-2}$ & Nienow et al., 1996 \\
SCABA-RT & $N \theta=5.6\left(\frac{P_{S C}}{V}\right)^{-0.25}\left(\frac{P_{R T}}{V}\right)^{-0.08}$ & John et al., 1998 \\
\hline
\end{tabular}

\subsubsection{Mixing time measurement techniques}

Several methods can be applied to measure the mixing time (Kraume and Zehner, 2001). These include conductivity (Bouaifi and Roustan, 2001; Raghav Rao and Joshi, 1988), pH meter (Guillard et al., 2000; Merchuk et al., 1998; Brennan \& Lehrer, 1976), colored dye addition method (Ascanio et al., 2002), radioactive liquid tracer (Pant et al., 2001), thermal response technique (Rewatkar et al., 1991) decolorization (Cabaret et al., 2008; Kuzmanic' and Ljubicǐ c,' 2001; Delaplace et al., 2000), colorimetry (Foucault et al., 2006; Ascanio et al., 2002), planar laser-induced fluorescence (Chung et al., 2007), thermography (Lee \& Yianneskis, 1997), and electrical resistance tomography (ERT) (Kazemzadeh 2016a, 2016b, 2017; Pakzad et al., 2013a, 2013b). Some of the most utilized methods' advantages and disadvantages are summarized in Table 2.5-2.

Table (2.5- 2). Advantages and disadvantages of mixing time measurement techniques. 


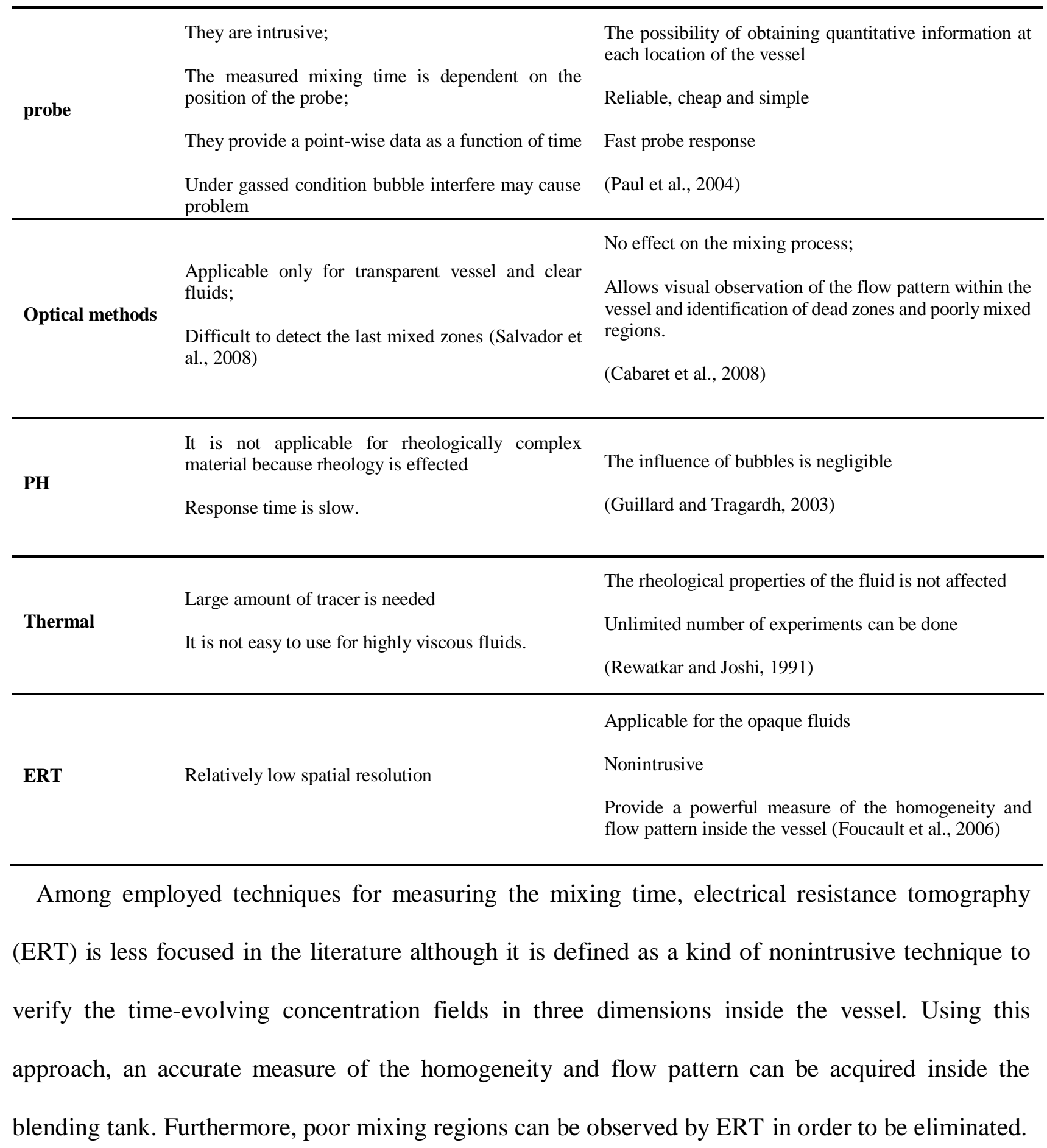


Chapter 2: Literature Review

\subsection{Bubble size distribution}

In the stirred vessel the formation of the bubbles and their subsequent rise due to the buoyancy force are the significant phenomena, which are related to the hydrodynamics of the gas-liquid flow in these devices. The development of the extremely complex hydrodynamics in the aerated mixing vessels depends not only on the liquid flow pattern and its interaction with the gas phase but also on the behavior of the bubbles and their related phenomena including coalescence and breakup. Besides, the gas-liquid mass transfer rate, which is a function of the interfacial area between the gas and liquid phases, is defined as a prevalent rate-limiting step in agitated tanks. The interfacial area between the gas and liquid phases depends on the overall gas holdup and the bubble diameter, which in turn rely on the local values of the bubble size and gas holdup. In addition, the bubble size dictates the rise velocity of the bubbles, which can affect the overall turbulence in the vessel and furthermore the efficiency of the gas-liquid contactors. Therefore, in order to improve the performance of the gasliquid mixing vessels, the detailed knowledge of the local bubble size distribution is crucial. A number of works have been conducted to investigate the effect of hydrodynamics of the agitated tank on the bubble size distribution, bubble rising velocity, and gas holdup (Bouaifi and Roustan, 2001; Hassan and Robinson, 1977).

The measurement of the local bubble size for the turbulent gas-liquid flow inside the aerated vessels is a difficult task and can be tackled by using diverse experimental approaches such as the optical imaging methods (Machon et al., 1997; Hirata et al., 1994), photographing techniques (Vlaev and Martinov, 1998; Andrew 1982), suction probes (Barigou and Greaves, 1991; Greaves and Kobbacy, 1984), and particle image velocimetry (PIV) (Chung et al., 2009; Pacek et al., 1998). The aforementioned measurement techniques show some restrictions including applicability in clear fluids, interfering with the flow inside the vessel, and measuring the global bubble characteristics 


\section{Chapter 2: Literature Review}

rather than the local ones. The visual tools such as photography/video techniques can measure the bubble size only in the vicinity of the tank wall. The conductivity probe is an intrusive technique and can only provide the information about the small area where it has been installed. Therefore, a timeconsuming procedure is required to obtain data from different parts of the system using the probes (Busciglio et al., 2013). Significant differences between the local and global results have been reported in the literature by utilizing the intrusive probes (Wachi et al., 1987). In the recent years, the non-invasive electrical resistance tomography (ERT) has been utilized to investigate the hydrodynamics and flow patterns of the gas-liquid contactors especially the bubble columns (Babaei et al., 2015; Hamood-Ur-Rehman et al., 2012; Jin et al., 2007; Fransolet et al., 2001; Wang et al., 2000). The fast speed of the ERT system in capturing the real-time data of the extremely fluctuating flow in the gas-liquid systems is the most important factor differentiating the ERT system from the other employed experimental procedures. In addition, ERT is capable of measuring the bubble size in any location inside the vessel without interrupting the developed flow. The accuracy of the ERT data has been confirmed in the literature by comparing the data obtained from ERT with those from other types of measuring techniques (Razzak et al., 2010, 2009; Fransolet et al., 2001). The applications of ERT in chemical engineering have been discussed by Sharifi and Young (2013). The ERT data enable us to employ the dynamic gas disengagement technique (DGD), which has been widely adopted in the literature to analyze the bubble rise velocity, bubble size, and gas holdup. While the DGD technique has been utilized extensively to study the hydrodynamics of the bubble columns (Babaei et al., 2015; Jin et al., 2007; Fransolet et al., 2001; Wang et al., 2000), little information is available in the open literature regarding the application of this technique in the aerated mixing vessels (Cooke et al., 2008). 
Chapter 2: Literature Review

\subsection{Numerical modeling of stirred vessel}

To analyze the fluid dynamics behaviour of the mechanically stirred vessel, use of sole experimental studies is quite time-consuming and constrained by a limited range of variables. Hence, computational fluid dynamics (CFD) method was adopted to gain detailed information about developed hydrodynamics within the stirred vessels. Developing CFD modelling for mixing vessel has been a center of attention for decades. Computational fluid dynamics (CFD) has already shown to be successful in simulating single phase mixers with various configurations (Ranade, 2002). Gasliquid flow in the mixing systems shows much more complexities in comparison with the singlephase ones, which makes the CFD simulation a tough task. Despite these difficulties, a number of CFD works on aerated mixing systems have been reported in the literature (Sarkar et al., 2016; Montante et al., 2008; Scargiali et al., 2007; Laakkonen et al., 2007; Khopkar et al., 2007, 2005; Kerdouss et al., 2006; Ranade and Deshpande, 1999; Bakker et al., 1995). Several challenging aspects are still present in the CFD simulation of the aerated mixing vessel including turbulence model, and interphase drag and non-drag force models which deal with momentum transfer between gas and liquid phases. A number of works have been reported on comparing different turbulent models, and a comprehensive study was conducted by Joshi et al. (2011).

A number of researches also have been carried out to investigate the effect of interphase forces include drag force, lift force, virtual mass force, turbulence dispersion force, and wall lubrication force on the CFD results (Ayeni et al., 2016; Scargiali et al., 2007; Kerdouss et al., 2006; Bakker, 1992). Most of them came to the conclusion that the drag force is the main contributor among the other considered forces (Lane et al., 2002). It should be mentioned that the drag coefficient, $C_{D}$, is an important factor involved in calculating the drag force, which has significant influence on the gas 
holdup. Different correlations have been proposed in open literature such as Grace drag model (1976), Ishii-Zuber drag model (1979), and Tomiyama drag model (1998). All of the aforementioned models calculate the drag coefficient based on the bubble shape. In addition, these three model were derived for stagnant fluids. However, in the aerated mixing vessels the interphase drag coefficient is much more complicated than that in the stagnant liquid. Therefore, most of the studies recommended use of a turbulence correlation factor proposed by Brucato et al. (1998), which was employed in this work.

As mentioned before, the bubble behavior in the aerated mixing vessel is extremely important particularly in conveying oxygen from the gas phase to the liquid phase, which is indispensable in bioreactors. Based on the CFD works carried out on the gas-liquid characteristics in the stirred vessel, it was demonstrated that single bubble size assumption may cause deviations between the predicted results and the observed data. Therefore, population balance model in conjunction with computational fluid dynamics have been used to analyze the local bubble size distribution within the gas-liquid agitated vessel (Azargoshasb et al., 2016; Wang et a., 2014; Ranganathan and Sivaraman, 2011; Petitti et al., 2010, 2013; Venneker et al., 2002).

\subsection{Research Objectives}

Our comprehensive literature review demonstrated that although the aerated coaxial mixers can be a promising approach to tackle the problems occurred in the traditional mixing vessels, no work has been reported on the use of the coaxial mixers for the gas-liquid mixing systems in the open literature. Therefore, the main objective of this thesis is to analyze the gas-liquid flow in a vessel equipped with a coaxial mixer composed of a central impeller and a wall scraping anchor through experimental and numerical studies. The main contributions of the current work are: 
Chapter 2: Literature Review

- Evaluating the effects of different design parameters including central impeller type, speed ratio, gas flow rate, rotation mode (co-rotating or counter-rotating), and fluid viscosity on the mixing time, and the local and global gas holdup of the aerated coaxial mixer through tomography technique.

- Finding the individual and interactive effects of the central impeller speed, anchor speed, gas flow rate, and viscosity on the mixing time using the response surface methodology.

- Analyzing the power uptake of the aerated coaxial vessel under various operating conditions including central impeller type, speed ratio, rotation modes, and viscosity.

- Developing a new generalized gas flow number and power number to obtain a master power curve for the aerated coaxial vessel.

- Investigating the main bubble characteristics such as number of bubble size classes, contribution of each class of bubble in the overall gas holdup, and bubble size throughout the aerated coaxial mixing vessel by means of the dynamic gas disengagement (DGD) technique coupled with tomography.

- Developing a CFD model to study the effects of the speed ratio, rotation mode, and viscosity on the fluid flow, turbulent kinetic energy, and gas holdup distribution in the aerated coaxial vessel.

- Developing a combined CFD-PBM (population balance modelling) model to assess the local bubble size distribution, bubble breakup, and coalescence. 


\section{Chapter 3}

\section{Experimental Setup and Procedures}

The current chapter deals with the experimental conditions, experimental set-up, experimental procedure, fluid properties, and error analysis.

\section{1 Experimental Setup}

The details of experimental setup are shown in Figure 3.1-1. The agitation tank designed and employed in the current work was a cylindrical vessel with a flat bottom. The diameter $(T)$ and the height of the vessel were $0.4 \mathrm{~m}$ and $0.61 \mathrm{~m}$, respectively. The liquid height was the same as the vessel diameter, so that the working volume was about $0.05 \mathrm{~m}^{3}$. The vessel was furnished by a central impeller and an anchor impeller stirred independently at different speeds. The central impeller was attached to the top shaft running at the speeds of 0-200 rpm while the anchor impeller was supported with the bottom shaft rotating at the speeds of 0-50 rpm. Two independent driven 
Chapter 3: Experimental Setup and procedures

motors, 1.5-hp and 1-hp, were employed to rotate the central and anchor impellers, respectively. Both motors were equipped with the variable frequency drives (AC Technology Corporation, USA) to control the rotational speed of the impellers. The torque was measured for both impellers independently using two rotary torque sensors (S. Himmelstein and Company, USA). The central impeller was four $45^{0}$ pitched blade turbine with $0.18 \mathrm{~m}$ diameter $\left(D_{c}\right)$ and $0.18 \mathrm{~m}$ off-bottom clearance $\left(C_{c}\right)$ in the upward and downward modes. The proximity impeller was anchor with the bottom and side clearance of $0.02 \mathrm{~m}$. Gas was fed into the mixing vessel by a ring sparger, which had a diameter of $144 \mathrm{~mm}\left(D_{s}=0.8 D_{c}\right)$ and was positioned $0.08 \mathrm{~m}$ under the central impeller. Air was supplied through 20 orifices, each $1 \mathrm{~mm}$ diameter. Air flow rate, which was in the range of 0 $120 \mathrm{SCFH}\left(0-0.056 \mathrm{~m}^{3} / \mathrm{min}\right)$, was measured by a rotameter. 
Chapter 3: Experimental Setup and procedures
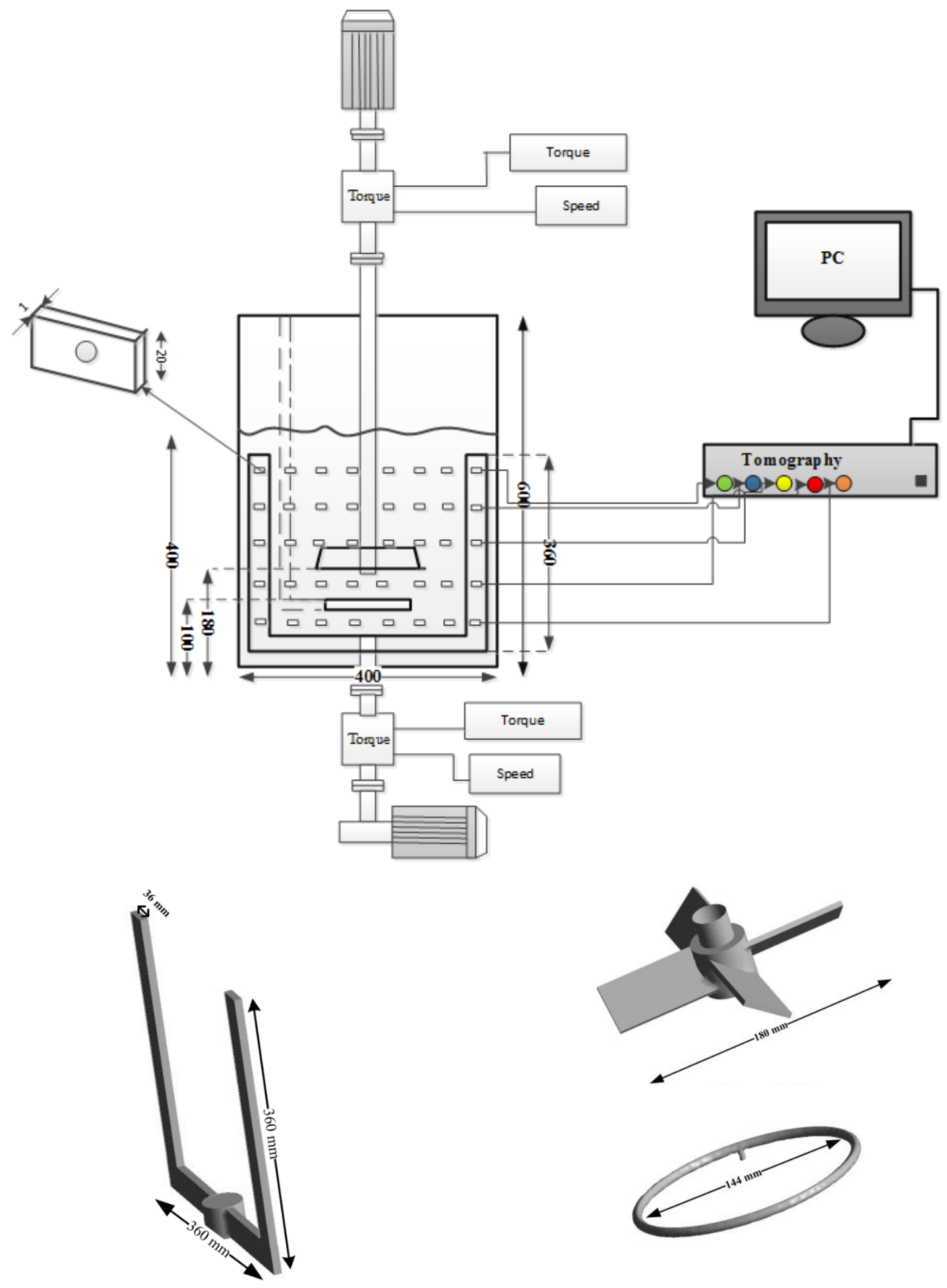

Figure (3.1- 1). Experimental set-up (dimensions in $\mathrm{mm}$ ) 
Chapter 3: Experimental Setup and procedures

\section{2 Fluid preparation}

The aqueous corn syrup solutions $(25,75$, and $90 \% \mathrm{wt})$ were used in the current study as the viscous Newtonian fluids. The viscosity of the corn syrup solution was measured at room temperature by a Bohlin CVOR Rheometer 150 (Malvern instruments, USA) using a concentric cylinders measuring system. The viscosity of the corn syrup solutions were 0.05 (low), 0.7 (medium), and 1.4 (high) Pa.s, and the density was 1280,1360 , and $1433 \mathrm{~kg} / \mathrm{m}^{3}$, respectively. Due to the intense temperature-dependence of the viscosity of corn syrup solution, the temperature of the working fluid was checked before and after each experiment using a thermocouple and it was in the range of 22 to $24.3^{\circ} \mathrm{C}$. The conductivities of the solution and brine were measured around 153 and $752 \mu \mathrm{S} / \mathrm{cm}$ using a conductivity meter and kept constant in all experiments.

\section{3 Measurement techniques}

\subsubsection{Electrical resistance tomography}

A non-invasive electrical resistance tomography (ERT) was applied for measurement of mixing time, gas holdup, and bubble size distribution. The conductivity distribution inside the mixing vessel can be visualized using ERT without the flow interruption. The tomography machine can determine the conductivity distribution within the tank based on the measurement of currents or voltages through a number of equally spaced electrodes on its boundaries. The ERT system employed in the current work has been depicted in Figure 3.1-1. A typical ERT system comprises electrodes, a data acquisition system (DAS), and a computer with image reconstruction software. The electrodes must be equally spaced at the specified intervals.

As shown in Figure 3.1-1, the vessel was fitted with five sensor planes; each comprised of 16 stainless steel electrodes attached at equal intervals around the boundary of the tank. Each 
Chapter 3: Experimental Setup and procedures

rectangular electrode was $20 \mathrm{~mm}$ high, $30 \mathrm{~mm}$ wide, and $1 \mathrm{~mm}$ thick. The ERT planes were numbered from top downward. The positions of the planes were shown in Figure 3.3-1.

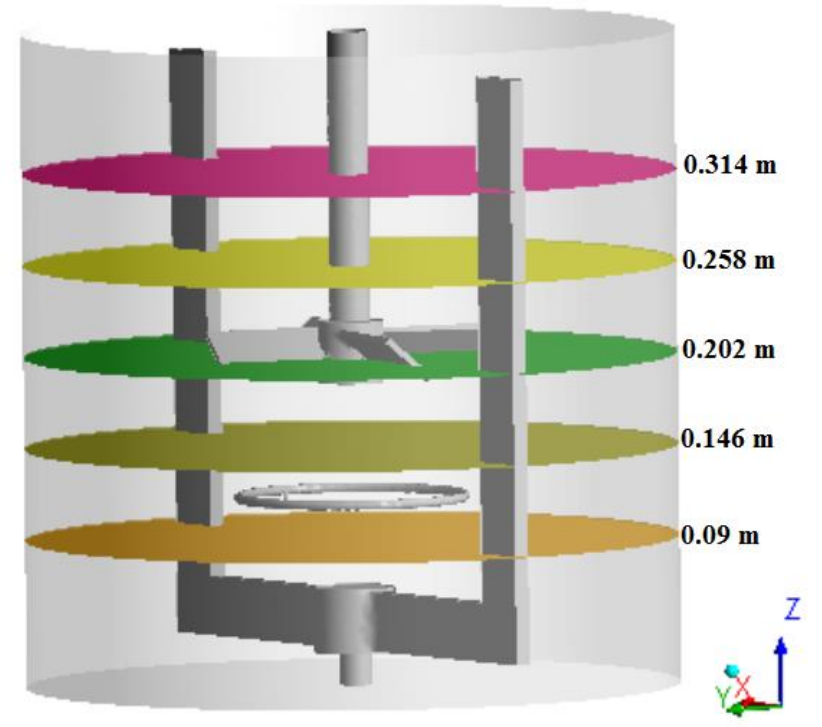

Figure (3.3- 1). The arrangement of the electrical resistance tomography sensor planes, named top downwad.

The electrodes were connected to a data acquisition system (DAS), which was connected to a PC by a USB 2.0 cable. DAS is a part of ERT system which injects current and gathers the quantitative data, which are used to determine the conductivity distribution inside the vessel. The adjacent strategy is the most common data collection strategy and was applied in this study. The reason is attributed to the fast image construction and minimum hardware requirements. In the aforementioned strategy, DAS injects current between two neighboring electrodes and measures the resultant voltage through all other neighboring electrodes except the current injecting ones. The driving pair is switched to the next electrode pair and the process of injecting current to adjacent electrodes is repeated until all independent pairs have been covered (Mann et al., 1997a; Pakzad et 
Chapter 3: Experimental Setup and procedures

al.,2008a). The number of the voltage measurements depends on the number of electrodes per plane, $N_{e}\left(N_{e}-3\right) / 2$, and in the current work is equal to 104 , where $N_{e}$ is the number of electrodes.

An image reconstruction algorithm is required to process the obtained data from electrodes mounted on the periphery of the vessel and determine the conductivity distribution within the vessel. Two different algorithms have been developed to process the acquired data including noniterative (Madupu et al., 2005) and iterative (Wang, 2002). A non-iterative image reconstruction algorithm (linear back projection-LBP) was used to convert the measured voltage data into 2D/3D conductivity image of each plane (see Figure 3.3-3). This algorithm has low computational demands in comparison with the iterative one. It should be mentioned that before measuring, calibrating the ERT system and taking reference data are required.

The tomography parameters applied in this study are given in Table 3.3-1. The parameters in this table were selected based on the preliminary experiments in order to reduce the noise.

Table (3.3- 1). Tomography parameters applied in this study.

\begin{tabular}{lc}
\hline Specification & Value \\
\hline Sampling time interval $[\mathrm{ms}]$ & 20 \\
Injection current $[\mathrm{mA}]$ & $15-75$ \\
Frequency of DAS [Hz] & $19200-38400$ \\
Maximum number of frames & $150-300$ \\
Frame per download & $150-300$ \\
\hline
\end{tabular}

\subsubsection{Mixing Time measurement}

As mentioned before, ERT was used to measure the mixing time for the aerated coaxial mixer. Reference data were collected before conducting measurements in order to omit the effects of all 
Chapter 3: Experimental Setup and procedures

internal parts, impellers, shafts, and sparger. To improve the measurement quality, a multiple reference frame approach was used. To do this, 150 frames were considered and the averaged of them was utilized as the reference frame. The value of mixing time was measured by the injection of $30 \mathrm{~mL}$ of $10 \%$ brine at the top of plane 1 and about $4 \mathrm{~cm}$ under the liquid surface near the shaft. All injections were done with a syringe. Using ERT, the air-corn syrup conductivity per time was obtained at five planes, which were numbered top downward. The conductivity of aerated corn syrup became stable after 19 s of air sparging, at which brine was injected. As depicted in Figure 3.3-2, the mixing time was measured as the time between the injection time and the time at which the mean conductivities of all planes reached to $95 \%$ of the steady-state value. It should be mentioned that three conductivity measurements were conducted for each experiment and the maximum standard deviation of $5 \%$ was achieved. Average obtained mixing time was calculated for each experiment.

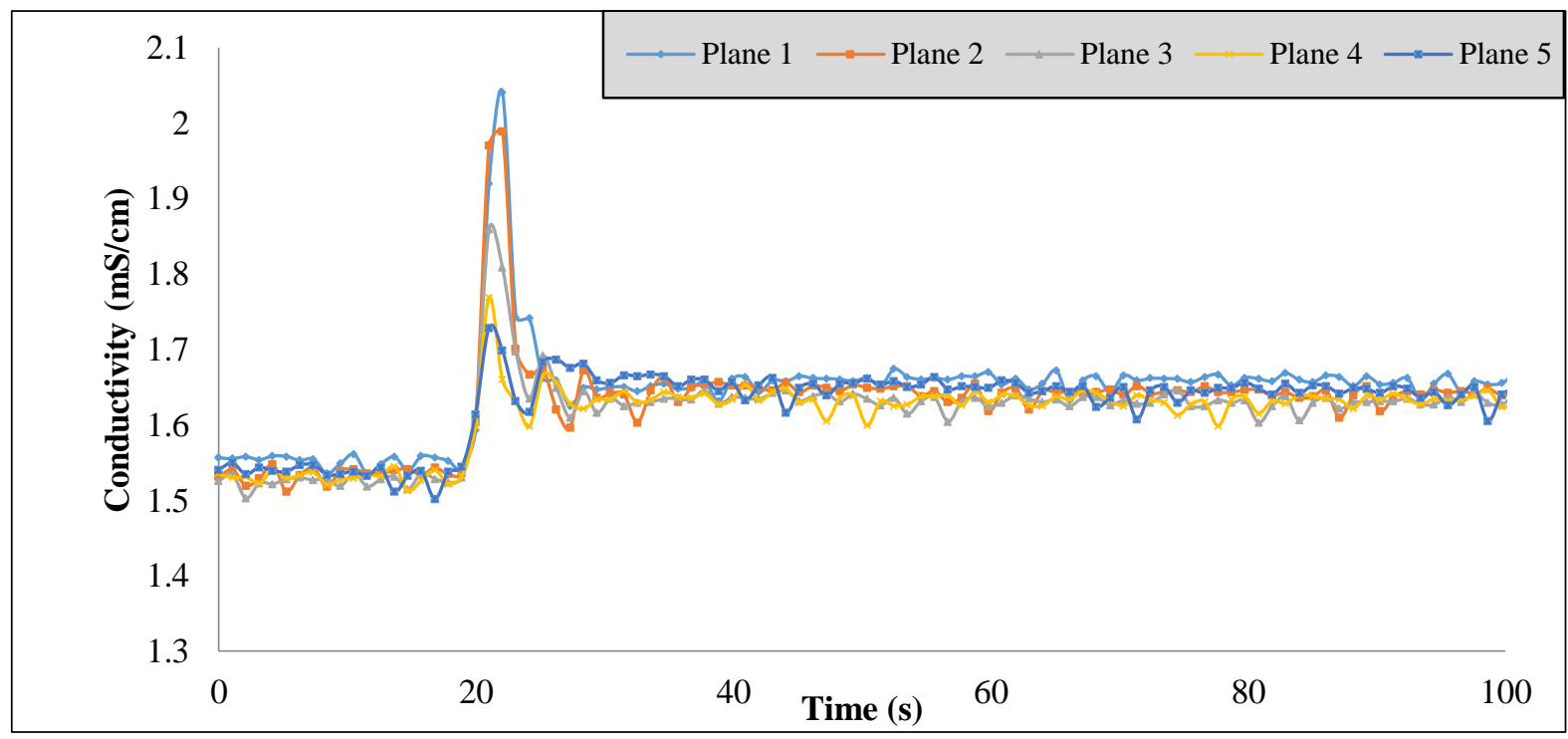


Chapter 3: Experimental Setup and procedures

Figure (3.3- 2). Mean Conductivity data versus time for corn syrup solution agitated by PBUanchor aerated coaxial mixer at $N_{a}=10 \mathrm{rpm}, N_{c}=90 \mathrm{rpm}$ and gas flow rate $0.0094 \mathrm{~m}^{3} / \mathrm{min}$ using ERT.

To visualize the flow pattern inside the aerated coaxial mixer, Figure 3.3-3 provides series of 2D contours and 3D images obtained from ERT after injecting $30 \mathrm{~mL}$ of the tracer into the aerated Newtonian corn syrup. The basic ideas of colors in the tomogram are to interpret the conductivity distribution within the vessel. In this study, corn syrup solution was conductive and air (gas phase) was non-conductive. Therefore, blue color means regions with less conductivity, air, while red color shows higher conductivity regions. Figure 3.3-3 exhibited the results for the experiment conducted at $N_{c}=135 \mathrm{rpm}, N_{a}=15 \mathrm{rpm}$, and gas flow rate $0.0283 \mathrm{~m}^{3} / \mathrm{min}$ for PBU-anchor coaxial mixer. After $2.1 \mathrm{~s}$ of injection, brine traveled downward and increased the conductivities of the other planes especially planes 2 and 3 . The axial flow generated by the central impeller discharged the downward travelling tracer to the bottom of the tank and its walls. At $t=6.3 \mathrm{~s}$, the tomographic images of all planes changed to green, representing the distribution of saline solution in the whole vessel. The $95 \%$ homogeneity was achieved after $6.3 \mathrm{~s}$ of injection. 
Chapter 3: Experimental Setup and procedures
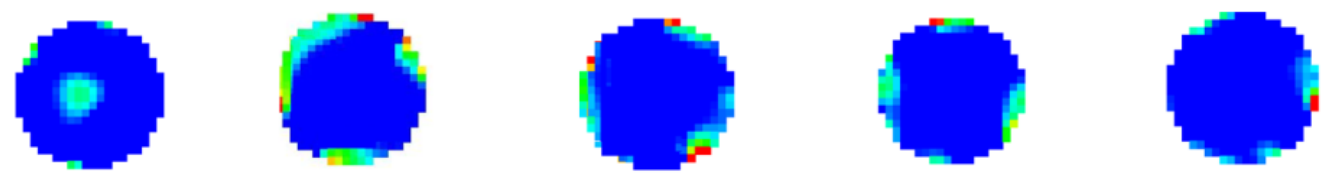

Before injection
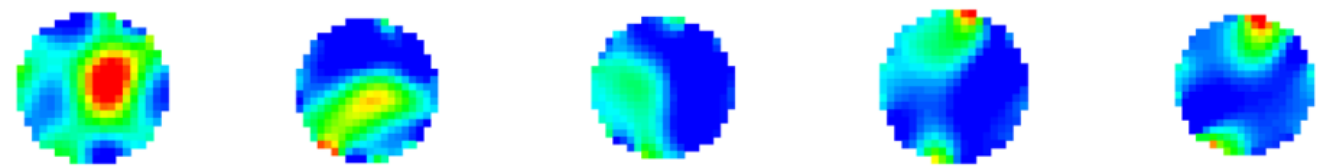

Plane 1

Plane 2

Plane 3

Plane 4

Plane 5

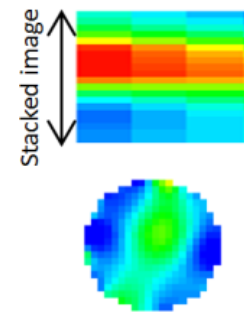

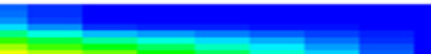
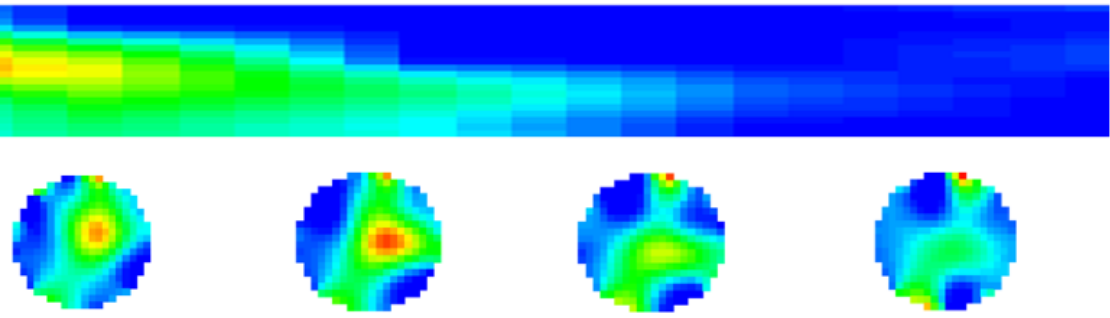

Plane 1

Plane 2

Plane 3

Plane 4

Plane 5

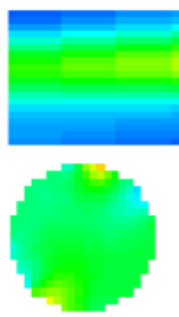

Plane 1

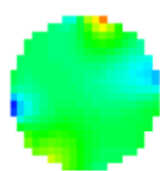

Plane 2

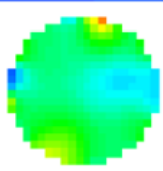

Plane 3

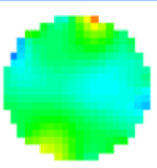

Plane 4

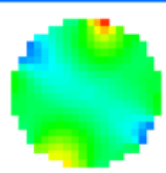

Plane 5
Time: After $2.1 \mathrm{~s}$ of injection (b)

Time: After $6.3 \mathrm{~s}$ of injection

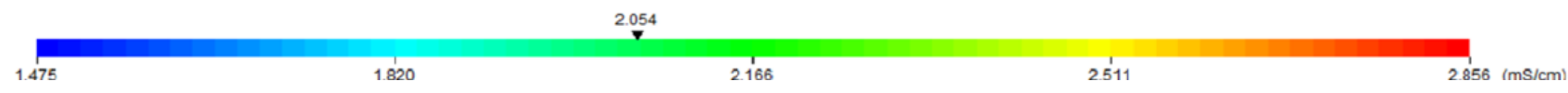

Figure (3.3- 3). 2D, 3D, and stacked tomography images showing the level of tracer homogeneity for corn syrup solution agitated by PBU-anchor at $\mathrm{Re}=1757$ and gas flow rate $0.0283 \mathrm{~m}^{3} / \mathrm{min}$ (Plane $1:(\mathrm{z}=0.314$ $\mathrm{m})$, Plane 2: $(\mathrm{z}=0.258 \mathrm{~m})$, Plane 3: $(\mathrm{z}=0.202 \mathrm{~m})$, Plane 4: $(\mathrm{z}=0.146 \mathrm{~m})$, and Plane 5: $(\mathrm{z}=0.090 \mathrm{~m}))$. 
Chapter 3: Experimental Setup and procedures

\subsubsection{Gas holdup measurement}

Gas holdup, gas volume fraction, is defined as a significant hydrodynamic parameter for evaluating the gas-liquid contact in the aerated stirred tanks. Both interfacial area and mass transfer rate are dependent on the gas holdup. Flow field in the vessel and energy dissipation rates also are governed by the gas volume fraction. Therefore, studying and measuring gas holdup are critical for designing and scaling up of mechanically agitated vessels.

The non-intrusive electrical resistance tomography (ERT) method enables us to investigate the hydrodynamic and flow pattern inside the vessel without affecting the developed flow. Therefore, in the current study the ERT system (Model P2+, Industrial Tomography Systems Plc., Manchester, UK) was used to measure the overall and local gas holdup values within the aerated coaxial mixer. As shown in Figure 3.3-4, the vessel was fitted with four sensor planes to obtain experimental gas holdup values. It should be mentioned that experiments were carried out for the sparged gas flow rates range from 0.0094 to $0.0469 \mathrm{~m}^{3} / \mathrm{min}$, which were chosen based on the literature review. The characteristics of the employed ERT system were explained in Table 3.3-1. 


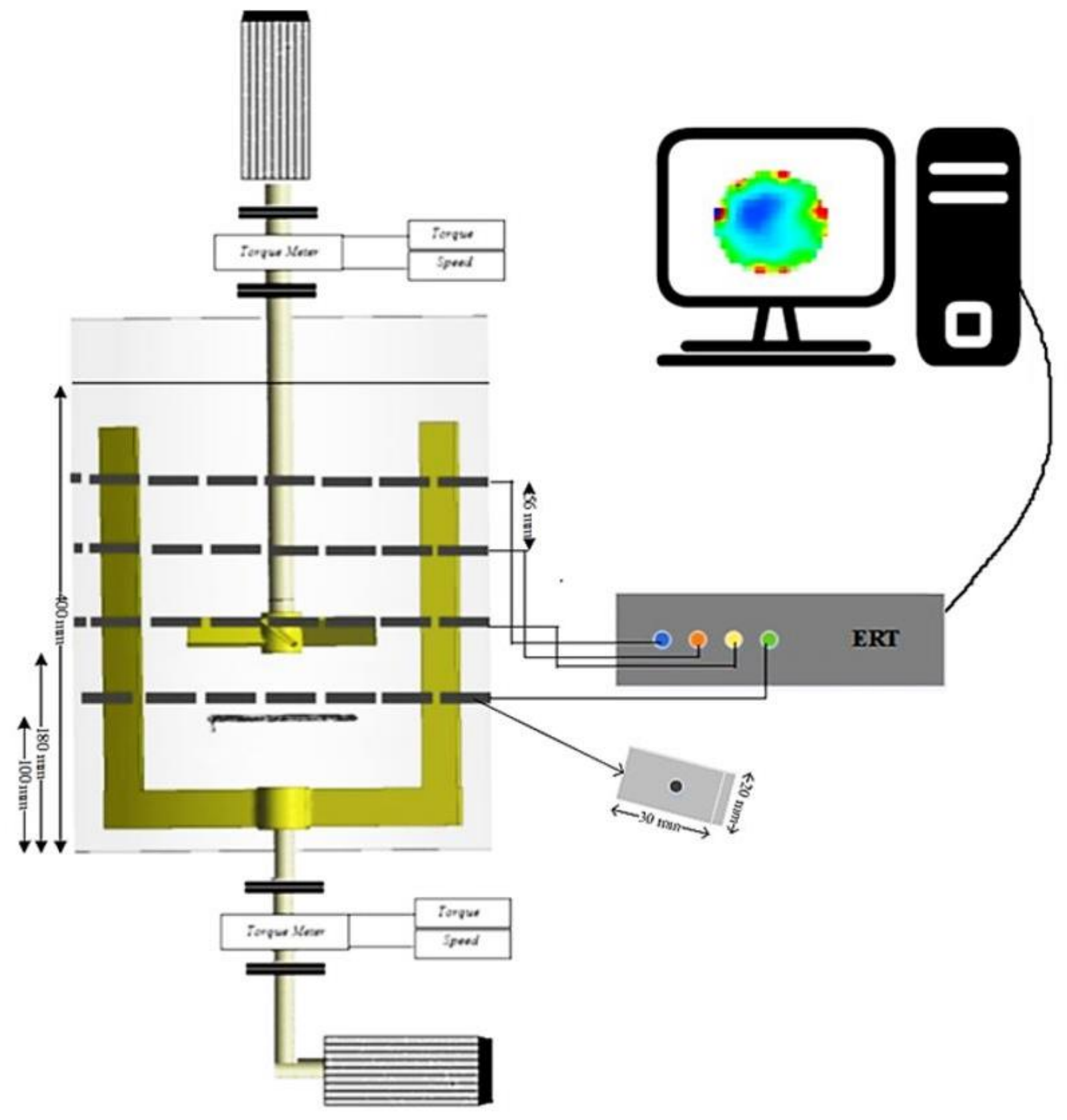

Figure (3.3- 4). Experimental setup for measuring gas holdup using ERT. 
Chapter 3: Experimental Setup and procedures

By employing ERT, the local conductivity distribution of the fluid being stirred by the coaxial mixer can be obtained. The measured local conductivities were converted to the local gas holdup values based on Maxwell's relation (Maxwell, 1881):

$$
\phi_{g}=\frac{2 \sigma_{l}+\sigma_{g}-2 \sigma_{m c}-\frac{\sigma_{m c} \sigma_{g}}{\sigma_{l}}}{\sigma_{m c}-\frac{\sigma_{g}}{\sigma_{l}} \sigma_{m c}+2\left(\sigma_{l}-\sigma_{g}\right)}
$$

where $\sigma_{l}$ and $\sigma_{g}$ are the conductivities of the continuous and dispersed phases, respectively, and $\sigma_{m c}$ is the local conductivity determined by ERT. Since the dispersed phase is non-conductive ( $\left.\sigma_{g}=0\right)$ the Maxwell equation is simplified as follows:

$$
\phi_{g}=\frac{2\left(\sigma_{l}-\sigma_{m c}\right)}{2 \sigma_{l}+\sigma_{m c}}
$$

The conductivity of the liquid phase $\left(\sigma_{l}\right)$ was obtained through a conductivity meter prior to the tomography measurements. Same as the procedure employed for measuring the mixing time, the reference data were acquired ahead of the measurements in order to omit the effects of impellers, shafts, and sparger. A multiple reference frame was employed to enhance the measurement accuracy. To do this, 100 frames were considered and the averaged of them was utilized as the reference frame. Due to the temperature-dependence of the viscosity of corn syrup solution, its temperature was checked before and after each experiment. The tomography images were employed to visualize the radial distribution of the gas throughout the aerated coaxial mixer. Figure 3.3-5 provides series of 2D images obtained from ERT after sparging the gas into the aerated corn syrup. It should be mentioned that each 2D tomogram was constructed using 316 pixels. The spatial 
Chapter 3: Experimental Setup and procedures

resolution of the tomography system utilized in this study was $10 \%$ of the tank diameter (Patel et al., 2014; Pakzad et al., 2013c, 2013d). In current work, corn syrup solution was conductive and air was non-conductive. Therefore, the blue color means regions with less conductivity (i.e. the higher gas percentage) while the red color shows the higher conductivity regions. Figure 3.3-5 exhibits the results for the experiment conducted at $N_{c}=180 \mathrm{rpm}, N_{a}=10 \mathrm{rpm}$, and gas flow rate 0.0283 $\mathrm{m}^{3} / \mathrm{min}$ for PBU-anchor coaxial mixer. As it can be seen the aforementioned operating conditions corresponded to the complete gas dispersion flow pattern, at which gas is distributed throughout the whole vessel.

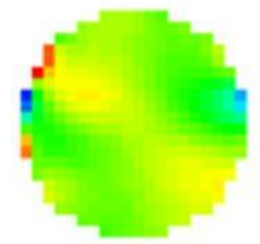

Plane 1

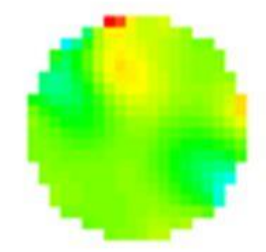

Plane 2

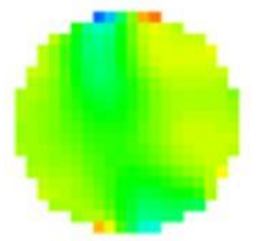

Plane 3

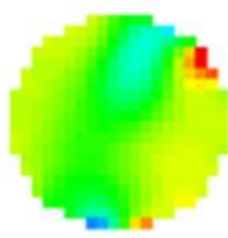

Plane 4

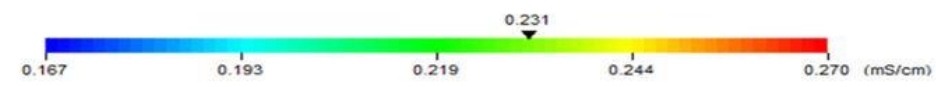

Figure (3.3- 5). Tomograms showing the conductivity distributions within the aerated PBUanchor coaxial mixer: $=\boldsymbol{N}_{c}=180 \mathrm{rpm}, \boldsymbol{N}_{a}=10 \mathrm{rpm}, \boldsymbol{Q}_{g}=0.0283 \mathrm{~m}^{3} / \mathrm{min}$, and $\mu=1.4 \mathrm{~Pa} \mathrm{~s}$.

\subsubsection{Dynamic Gas Disengagement Technique procedure}

Jin et al. (2007) first developed a combination of electrical resistance tomography and dynamic gas disengagement technique to predict the bubble behavior in the bubble column. In the current work the same procedure was applied to estimate the number of bubble classes in the aerated coaxial mixing vessel. It should be mentioned that all the measurements were done at the complete dispersion of the bubble throughout the vessel in order to consider that the distribution of the 
Chapter 3: Experimental Setup and procedures

bubbles is axially homogenous at the moment when the gas flow was cut off. In addition, the cross sectional area occupied by ascending bubbles was fixed. The previous researchers, who employed the DGD technique, assumed that there were no bubble-bubble interactions while in this paper the breakup and coalescence caused by the rotating impellers in the vessel were considered.

Because of the interaction between the rising bubbles and the rotation of both impellers (the central and the anchor impellers) in the aerated coaxial mixing vessel, M-bubble size classes (multimodel distribution) may exist at different heights of the vessel. Therefore, two sensor planes (plane 2 and plane 4) were employed; one beneath and one above the central impeller, see Figure 3.3-4. The duration of the gas disengagement after ceasing the gas flow ( $\mathrm{t}=0 \mathrm{~s})$ can be divided into $\mathrm{M}$ straight lines with different slopes separating various bubble classes as it can be seen in Figure 3.36. Generally, bubbles with different sizes disengage with different velocities. Therefore, the larger the bubbles, the faster they disengage. For instance, $\mathrm{t}=1 \mathrm{~s}$ relates to the largest bubble disengagement. During the disengagement process, smaller bubbles travel upward slower, whereas, $(t=3 \mathrm{~s}$ for plane 2 and $t=4 \mathrm{~s}$ for plane 4$)$ corresponds to the last stage of the gas disengagement of the smallest bubbles. Accordingly, during the DGD process and after shutting off the gas supply, the gas holdup began to descent in the aerated coaxial mixing vessel. In order to measure the reduction of the gas holdup within the DGD process, Maxwell's relation (Eq. (3.3-2)) was employed. Using Maxwell's correlation, the acquired conductivity data from ERT system were converted to the gas holdup values.

In the aerated coaxial mixing vessel with different class of bubbles the overall gas holdup can be calculated as the summation of the occupied holdup by each class of bubble (Jin et al., 2007): 
Chapter 3: Experimental Setup and procedures

$$
\phi_{\text {g-overral }}=\sum_{j=1}^{M} \phi_{j g}
$$

here $\mathrm{M}$ is the number of bubble size classes at the aerated coaxial mixing vessel and $\phi_{j g}$ is specified as the gas holdup of class $\mathrm{j}$.

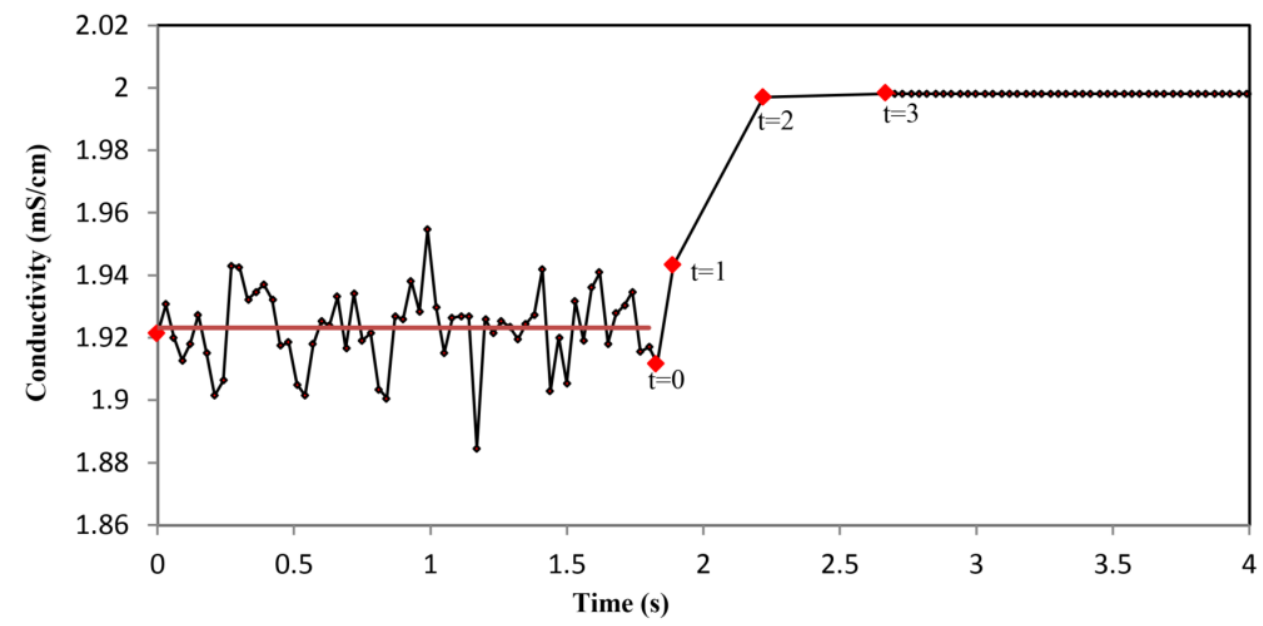

(a)

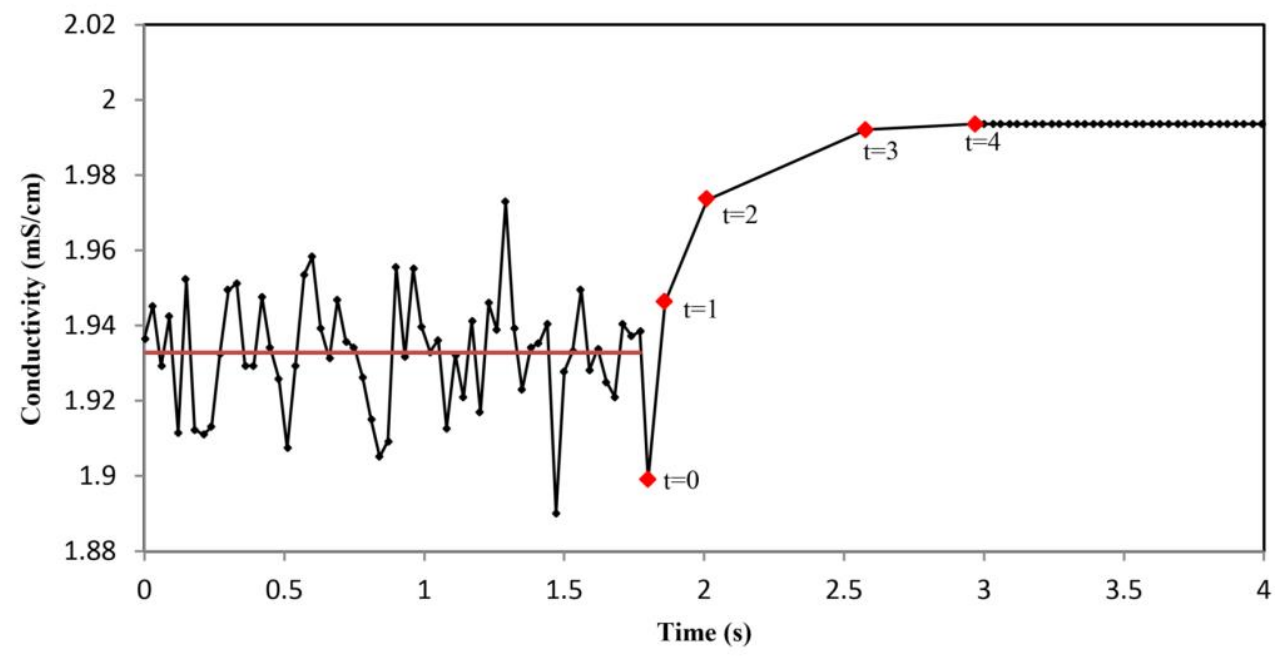

(b)

Figure (3.3- 6). Gas disengagement profiles of the aerated coaxial mixing vessel at $\mathrm{N}_{c}=150 \mathrm{rpm}$ $\mathrm{N}_{\mathrm{a}}=9.4 \mathrm{rpm}$, and $\mathrm{u}_{\mathrm{g}}=0.15 \mathrm{~m} / \mathrm{s}$ : (a) plane $2(\mathrm{z}=0.258 \mathrm{~m})$ and (b) plane $4(\mathrm{z}=0.146 \mathrm{~m})$. 
In Figure 3.3-7, the DGD process is represented by a series of 2D images reconstructed by ERT for air-corn syrup coaxial mixing vessel $\left(u_{g}=0.15 \mathrm{~m} / \mathrm{s}, N_{c}=150 \mathrm{rpm}\right.$, and $\left.N_{a}=15 \mathrm{rpm}\right)$. The initial condition of the gas-liquid coaxial mixing vessel before stopping the aeration is depicted at $t=0 \mathrm{~s}$. As it can be seen, the gas phase was completely distributed inside the tank, resulted in complete dispersion regime. However, after cutting off the gas phase, the disengagement of the bubbles was commenced and the low conductivity region (blue region) was vanished and finally replaced by red color which indicated a vessel with only high conductive corn syrup liquid. It should be mentioned that the formation of high-conductivity regions in the middle of the tank in first three images $(t=0-0.3 \mathrm{~s})$ showed that the gas volume fraction was lower in these regions due to the presence of the shaft.

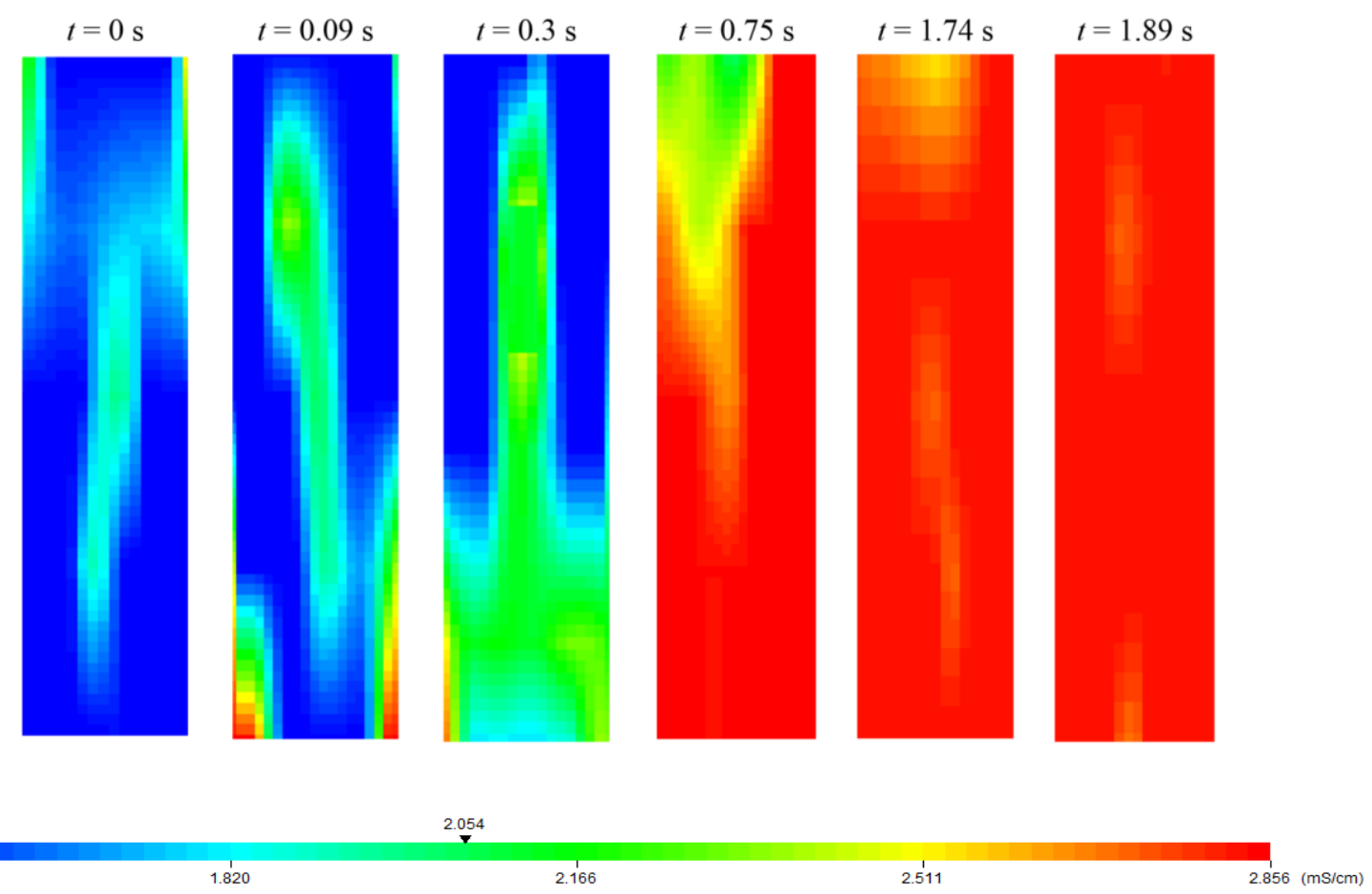

Figure (3.3- 6). Tomograms showing the level of the gas dispersion for corn syrup solution agitated by PBU-anchor at $N_{c}=150 \mathrm{rpm}, N_{a}=15 \mathrm{rpm}$, and $u_{g}=0.15 \mathrm{~m} / \mathrm{s}$. 
Chapter 3: Experimental Setup and procedures

\subsubsection{Power measurement}

To measure the actual gassed power uptake of the coaxial mixer, each shaft was furnished with a rotary torque sensor (S. Himmelstein and Company, USA). These torque meters provided the continuous torque measurement of both central and anchor impellers at different operating conditions. Since the residual torque of the motors was a part of the recorded torque, the displayed torque should be corrected by subtracting the torque of friction $\left(M_{\text {friction }}\right)$ from the displayed torque $\left(M_{\text {display }}\right)$ :

$$
M_{\text {corrected }}=M_{\text {display }}-M_{\text {friction }}
$$

To measure the friction torque, the shafts were rotated at different rotational speeds in the empty tank. Subsequently, torque of the central and anchor impellers were obtained at different speed ratios when the gas phase was sparged into the corn syrup solution. The gassed power uptake of each impeller was obtained by the following relation:

$$
P_{g-i}=2 \pi N_{i} M_{\text {corrected }-i}
$$

where $i$ is referred to the central (c) and anchor impeller (a), and $N$ is the rotational speed. Finally, the total gassed power of the coaxial mixer was calculated as the sum of the gassed power uptake of the central and close clearance impellers:

$$
P_{g t o t}=P_{g-c}+P_{g-a}
$$

\section{4 Experimental Design}

One of the main objectives of this study was the prediction of the mixing time for the coaxial mixers at different operating conditions. The list of variables and the maximum and minimum levels 
Chapter 3: Experimental Setup and procedures

at which they have been varied are summarized in Table 3.4-1. The critical ranges were chosen based on the preliminary mixing time experiments.

Table (3.4- 1). Independent variables and their actual values using Box-Behnken design.

\begin{tabular}{cccccc}
\hline Symbols & Variables & Units & Low level & Central Level & High level \\
\hline $\boldsymbol{X}_{\boldsymbol{I}}$ & Central impeller speed & $1 / \mathrm{s}$ & 1.5 & 2.25 & 3.00 \\
$\boldsymbol{X}_{\mathbf{2}}$ & Anchor speed & $1 / \mathrm{s}$ & 0.17 & 0.33 & 0.50 \\
& & & & & \\
$\boldsymbol{X}_{3}$ & Gas flow rate & $\mathrm{m}^{3} / \mathrm{min}$ & 0.0094 & 0.0283 & 0.0472 \\
$\boldsymbol{X}_{4}$ & Viscosity & Pa.s & 0.05 & 0.7 & 1.4 \\
\hline
\end{tabular}

Preliminary experiments also showed that the central impeller speed $\left(X_{1}\right)$, anchor speed $\left(X_{2}\right)$, gas flow rate $\left(X_{3}\right)$, and viscosity $\left(X_{4}\right)$ had great influence on the mixing time of the aerated coaxial mixers. Therefore, in this work, the impacts of these four factors were investigated. To achieve this goal, 29 experiments were performed using a four-factor three-level Box-Behnken design combined with response surface methodology. Three replicates at the center point were considered to evaluate the reproducibility of the method. The Box-Behnken design offers some advantages in comparison with the other experimental design such as requiring fewer experiment runs and optimizing the main, interaction, and quadratic effects. Table 3.4-2 indicates that a quadratic model, Eq. 3.4-1, can be a proper choice to correlate the dependent and independent variables.

$$
Y=\beta_{0}+\sum_{i=1}^{3} \beta_{i} X_{i}+\sum_{i=1}^{3} \beta_{i i} X_{i}^{2}+\sum_{i=1}^{3} \sum_{j=2}^{3} \beta_{i j} X_{i} X_{j}+\text { error }
$$

where $Y, \beta_{0}, \beta_{i}, \beta_{i i}$, and $\beta_{i j}$ are response (i.e. mixing time), regression coefficient for intercept, linear, quadratic and interaction terms, respectively. The response surface methodology was applied to the experimental data using Design-Expert (Design Expert software version 9). 3D surface plots 
Chapter 3: Experimental Setup and procedures

were plotted by varying two independent variables of quadratic model while the third factor was fixed at its center point.

Table (3.4- 2). Model summery statistics for mixing time.

\begin{tabular}{cccccc}
\hline Source & Std. Dev. & R-Squared & Adjusted R-Squared & Predicted R-Squared & Press \\
\hline Linear & 1.61 & 0.8976 & 0.8805 & 0.8414 & 96.64 \\
2FI & 1.60 & 0.9244 & 0.8825 & 0.7621 & 144.97 \\
Quadratic & 1.18 & 0.9680 & 0.9361 & 0.7960 & 124.19 \\
Cubic & 0.00 & 1.00 & 1.00 & - & + \\
\hline
\end{tabular}

\section{5 Error analysis}

The errors associated with torque sensors and ERT were analyzed as follows:

\subsubsection{Evaluation of Torque Sensor Precision}

The random error of the torque sensors (Models MCRT 48201V (2-2)-N-N and MCRT 48201V (1-2)N-N, S. Himmelstein and Company, USA) generated by electronic fluctuations, mechanical play, and friction can be specified using the standard deviation relation:

$$
S D=\sqrt{\frac{1}{N} \sum_{i=1}^{N}\left(x_{i}-\kappa\right)^{2}}, \text { where } \kappa=\frac{1}{N} \sum_{i=1}^{N} x_{i}
$$

where $N$ is the number of measurements, and $x_{i}$ is variable and $\kappa$ is the mean value of the measurements. It was found that the errors are small enough $(\mathrm{SD}<1 \%)$ to accept that the torque measurements were error independent.

\subsubsection{Evaluation of ERT Measurements Precision}

As mentioned in section 3.3.1.1, the ERT system was calibrated for each measurement. Each of the measurements was repeated three times and then the standard deviation was calculated. The 
Chapter 3: Experimental Setup and procedures

insignificant standard deviations $(\mathrm{SD}<5 \%)$ were observed confirming a good repeatability and reproducibility of the experiments.

\section{6 Experimental Conditions}

The effect of the central impeller type, central impeller speed, anchor speed, impeller speed ratio, viscosity, gas flow rate, and rotational mode on the agitation of the corn syrup solution through the aerated coaxial mixers were characterized in terms of the power consumption, mixing time, gas holdup, and bubble size distribution. The experimental conditions for this research work are summarized in Table (3.6-1).

Table (3.6- 1). Employed parameters in the current work.

\begin{tabular}{ll}
\hline Parameter & value \\
\hline Central impeller & Four bladed pitch turbine, Upward and downward, Lightnin (A310) \\
Close clearance impeller & Anchor \\
Central impeller rotational speeds (RPM) & $0-200$ \\
Close Clearance impeller speeds (RPM) & $0-50$ \\
Speed ratio (-) & $5,8,10,12,14$ \\
Sparger type & Ring \\
Number of holes & 20 \\
Holes diameter $(\mathbf{m m})$ & 1 \\
Rotation mode & Co-rotating, Counter-rotating \\
Gas flow rate (SFCM) & $20-120$ \\
Newtonian viscosity (Pa.s) & $0.05,0.7,1.4$ \\
Surface tension $(\mathbf{N} / \mathbf{m})$ & 0.08 \\
\hline
\end{tabular}




\section{Chapter 4}

\section{Computational Fluid Dynamics (CFD)}

Application of the CFD for simulation of the agitated vessels dates back to 1970 (Harris et al., 1996). The important aspect of CFD is that the time-consuming and expensive experimental techniques can be replaced by this approach. Besides, some of the apparatuses being used in the industry operate at high pressures and temperatures and deal with highly hazardous materials. Such a process is always challenging to reproduce in experiments. Using CFD, governing flow equations can be solved and used to predict the mixing performance of the industrial scale agitated vessel for which no empirical correlations exist.

Understanding of the complicated interactions between rotating impellers and bubbles are the main problems dealing with the gas-liquid stirred vessels, especially aerated coaxial mixing systems. In the last decades the rapid increase in computational capability and development of efficient numerical techniques have necessitated a detailed simulation of fluid flows. Several studies have been conducted to understand the complex hydrodynamics involved in the gas-liquid mixers. In 
Chapter 4: Model Development

the current study, a Computational Fluid Dynamics package called FLUENT (Version 16) was used to model the aerated coaxial mixing vessel.

\subsection{Model Development}

The fluid motion can be described by solving the conservation equations including mass, momentum, and energy. Considering the mixing system as an isothermal process, the governing equations consist of the mass and momentum balances. CFD solves the partial differential conservation equations and the final results can be obtained by the following steps:

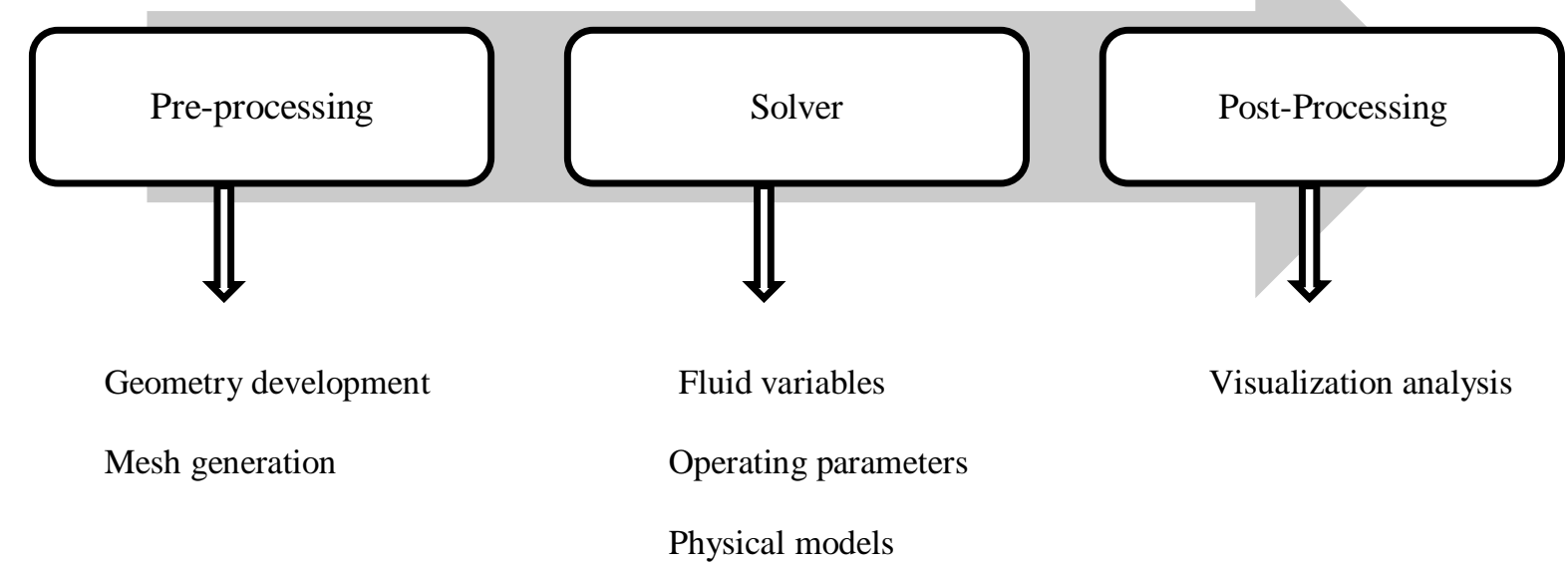

In pre-processing, the geometry (computational domain) is developed by determining the parts and dimensions of the equipment; at which governing equations are being solved. The mesh generation is the discretization of the domain into a number of small and non-overlapping subdomains (grids). In the solver step, using the defined boundary conditions and fluid properties, the governing equations are solved by appropriate numerical algorithm. Flow variables such as velocity, pressure, density, and concentration can be provided through the computed data. In the 


\section{Chapter 4: Model Development}

post processing (the flow visualization step), the obtained results from the solver can be reported and displayed in the various ways such as the vector and contour plots.

Two approaches have been reported in the literature for the numerical calculation of gas-liquid flows: Eulerian-Lagrangian and Eulerian-Eulerian. In the Eulerian-Lagrangian approach, fluid flow is assumed as the continuum and is solved by the Navier-Stokes equations while the gas phase is solved by tracking a large number of particles such as bubbles and droplets through the flow field. Eulerian-Eulerian is the most common approach to understand the flow behavior of gas and liquid phases (dispersed and continuous phases), at which two phases are treated as interpenetrating continua. Using this approach governing equations are solved independently for each phase.

Modelling of the aerated coaxial mixing vessel was conducted using the Eulerian-Eulerian twofluid model. By adopting the Eulerian-Eulerian approach, the summation of the volume fractions of two phases is considered as unity for all domains:

$$
\begin{aligned}
& \phi_{l}+\phi_{g}=1 \\
& \frac{\partial\left(\rho_{i} \phi_{i}\right)}{\partial t}+\nabla \cdot\left(\rho_{i} \phi_{i} \vec{u}_{i}\right)=0 \\
& \frac{\partial\left(\phi_{i} \rho_{i} \vec{u}_{i}\right)}{\partial t}+\nabla \cdot\left(\phi_{i} \rho_{i} \vec{u}_{i} \vec{u}_{i}\right)=-\phi_{i} \nabla p+\nabla \cdot\left(\tau_{\text {eff }}^{=}\right)+\phi_{i} \rho_{i} g+F_{i}
\end{aligned}
$$


where $\phi_{i}, \rho_{i}$, and $u_{i}$ are volume fraction, density, and mean velocity of the phase $i$ (liquid or gas). The pressure gradient, stress, gravity, and interphase momentum exchange terms are presented in the right hand side of the momentum equation (Eq. 4.1-3), respectively.

Most of the time, the flow regime occurs in the gas-liquid mixing systems is turbulent, which can be solved in average forms. Therefore, Reynolds stress tensor as a function of time-averaged velocity (based on Boussinesq's hypothesis) can be written as (Boussinesq, 1877):

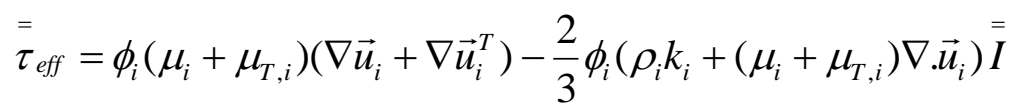

here $\mu_{i}$ and $\mu_{T, i}$ represent the laminar and turbulent viscosity of the phase $i$, respectively.

\subsubsection{Turbulence Closure}

In order to estimate the turbulent viscosity of the continuous phase (liquid phase), different turbulent models such as $k$-epsilon and $k$-omega can be employed. Among them, $k$-epsilon has been proven as an efficient model for describing the main features of turbulence in the agitated vessels (Montante et. al, 2008; Dhanasekharan et. al, 2005). Here RNG $k$-epsilon turbulence model was used for modelling of the aerated coaxial mixing vessel, due to its sufficient convergence (Azargoshasb et al., 2016). The assumption in the $k$-epsilon model is that the flow is totally turbulent and the effects of molecular viscosity are insignificant.

Turbulent viscosity of the liquid phase can be acquired from: 
Chapter 4: Model Development

$$
\mu_{T, m}=C_{\mu} \rho_{m} \frac{k^{2}}{\varepsilon}
$$

here $k$ and $\varepsilon$ are denoted as turbulent kinetic energy and energy dissipation rate which can be obtained by $k$-epsilon models as follows:

$$
\begin{aligned}
& \frac{\partial\left(\rho_{m} k\right)}{\partial t}+\nabla \cdot\left(\rho_{m} \vec{u}_{m} k\right)=\nabla \cdot\left(\frac{\mu_{T, m}}{\sigma_{k}} \nabla k\right)+P_{k, m}-\rho_{m} \varepsilon \\
& \left.\frac{\partial\left(\rho_{m} \varepsilon\right)}{\partial t}+\nabla \cdot\left(\rho_{m} \vec{u}_{m} \varepsilon\right)=\nabla \cdot\left(\frac{\mu_{T, m}}{\sigma_{\varepsilon}} \nabla \varepsilon\right)+C_{\varepsilon 1} P_{k}-C_{\varepsilon 2} \rho_{l} \varepsilon\right)
\end{aligned}
$$

where $P_{k}$ is the shear related production of turbulent kinetic energy which is formed because of mean velocity gradients and can be as (Wang et al., 2014):

$$
P_{k}=\mu_{T, l} \nabla \vec{u}_{l} \cdot\left(\nabla \vec{u}_{l}+\nabla \vec{u}_{l}^{T}\right)-\frac{2}{3} \nabla \cdot \vec{u}_{l}\left(3 \mu_{T, l} \nabla \cdot \vec{u}_{l}+\rho_{l} k\right)
$$

In order to take into account the turbulence, which is induced by the bubble movements in the liquid phase, the model proposed by Sato and Sekoguchi (1975) can be applied:

$$
\mu_{b u b, l}=C_{\mu p} \rho_{l} \phi_{g} d_{b}\left|\vec{u}_{g}-\vec{u}_{l}\right|
$$

The turbulent viscosity of the gas phase is obtained as: 


$$
\mu_{T, g}=\frac{\rho_{g}}{\rho_{l}} \mu_{T, l}
$$

The default constant values of the RNG- $k$-epsilon model can be given as: $C_{\mu}=0.0845, C_{\varepsilon 1}=1.42$, and $C_{\varepsilon 2}=1.68$.

\subsubsection{Interphase force}

The interaction between the liquid phase and the gas phase can be defined by the interphase force term, $F_{i}$, in Eq. (4.1-3). In fact, the motion of a bubble is affected by the different forces such as drag, lift, and virtual mass, which induced by the fluid. A number of studies have been conducted to investigate the effect of interphase forces include drag force, lift force, virtual mass force, turbulence dispersion force, and wall lubrication force on the CFD results (Ayeni et al., 2016; Scargiali et al., 2007; Kerdouss et al., 2006; Bakker, 1992). Most of them came to this conclusion that the drag force is the main contributor among the other considered forces (Lane et al., 2002). Therefore, only drag force was considered in the current study. The drag force has two sources: the surface friction on the surface of the rising bubble and non-uniform pressure distribution, because of slip velocity differences between gas and liquid phases, which is related to the shape of the bubble (Bird et al., 2002). The Drag force between gas phase and liquid phase was calculated by Eq. (4.1-11):

$$
F_{D}=\frac{3}{4} \frac{C_{D}}{d_{b}} \rho_{l} \phi_{g} \phi_{l}\left|\vec{u}_{g}-\vec{u}_{l}\right|\left(\vec{u}_{g}-\vec{u}_{l}\right)
$$


It should be mentioned that drag coefficient, $C_{D}$, is an important factor involved in calculating the drag force which has significant influence on the gas holdup. Different correlations have been proposed in open literature such as Grace drag model (1978), Ishii-Zuber drag model (1979), and Tomiyama drag model (1998). All of the aforementioned models calculate the drag coefficient based on the bubble shape. In addition, these three model were derived for the stagnant fluids. However, in the aerated mixing vessels the interphase drag coefficient is much more complicated than that in the stagnant liquid. Therefore, most of the studies recommended use of a turbulence correlation factor proposed by Brucato et al. (1998), which was employed in this work as follows:

$$
\frac{C_{D}-C_{D 0}}{C_{D 0}}=6.5 \times 10^{-6}\left(\frac{d_{b}}{\eta}\right)^{3}
$$

where $d_{b}$ and $\eta$ stand for the bubble diameter, and Kolmogorov length scale, respectively. $C_{D 0}$ is drag coefficient of a single bubble in stagnant liquid which can be calculated as:

$$
C_{D 0}=\max \left(\frac{24}{\mathrm{Re}_{b}}\left(1+0.15 \operatorname{Re}_{b}^{0.687}\right), \frac{8}{3} \frac{E_{0}}{E_{0}+4}\right)
$$

here $\operatorname{Re}_{b}$ and $E_{0}$ are the bubble Reynolds number, the ratio of inertia to viscous drag force, and Eotvos number (Yang et al. 2015).

\subsubsection{Population balance model}

One of the most important parameters, which can affect the interphase momentum exchange and mass transfer rate between the gas and liquid phases, is the size of the bubbles and their dispersion throughout the mixing vessel. Therefore, the prediction of the bubble size distribution 


\section{Chapter 4: Model Development}

is necessitated. The complex hydrodynamic phenomena occurring in the stirred vessel result in the bubble breakup and coalescence. The bubble breakup happens when the shear stresses developed by the eddy overcome the cohesive forces and the surface tension of the gas bubbles. On the other hand, the bubble coalescence happens when two neighboring bubbles merge because of the rupture of the liquid film which separates bubbles (Khopkar et al. 2005).

Bubble size distribution within the aerated coaxial mixer was predicted by Multiple Size Group (MUSIG) model associated with bubble breakage and coalescence kernels. In this work, fifteen bubble classes ranging from 0.5 to $12 \mathrm{~mm}$ (noticed in the conducted experiments at the same operating conditions) were considered. The discrete approach was employed, which represents the bubble population in terms of the number of bubble size bins or classes. Therefore, based on the aforementioned method, the fifteen bubble bins were: $0.01269,0.0100,0.0080,0.00635,0.00504$, $0.0040,0.00317,0.00252,0.0020,0.00159,0.00126,0.0010,0.00079,0.00063,0.00050 \mathrm{~m}$, respectively.

Population balance equations are summarized as follows:

$$
\begin{aligned}
& \frac{\partial}{\partial t}\left[n(V, t)+\nabla \cdot[U(V, t) n(V, t)]=S_{i}\right. \\
& S_{i}=B_{\text {aggregation }}-D_{\text {aggregation }}+B_{\text {breakage }}-D_{\text {breakage }} \\
& B_{\text {aggregation }}=0.5 \int_{0}^{V} Q\left(V-V^{\prime}, V^{\prime}\right) n\left(V-V^{\prime}, t\right) n\left(V^{\prime}, t\right) d V^{\prime} \\
& D_{\text {aggregation }}=\int_{0}^{\infty} Q\left(V, V^{\prime}\right) n(V, t) n\left(V^{\prime}, t\right) d V^{\prime}
\end{aligned}
$$




$$
\begin{aligned}
& B_{\text {breakage }}=\int v g\left(V^{\prime}\right) \beta\left(V \mid V^{\prime}\right) n\left(V^{\prime}, t\right) d V^{\prime} \\
& D_{\text {breakage }}=g\left(V^{\prime}\right) n(V, t)
\end{aligned}
$$

here $\boldsymbol{n}(\boldsymbol{V}, \boldsymbol{t})$ is the number density of bubbles with volume $\boldsymbol{V}$ at time $t$. $U(V, t)$ means the bubble velocity with volume $V$ at time $t . B_{\text {breakage }}, D_{\text {breakage }}, B_{\text {aggregation }}$, and $D_{\text {aggregation }}$ represent the birth rate due to breakup of the large size bubbles, the death rate because of breakup into smaller size bubbles, the birth rate due to coalescence of small size bubbles, and the death rate due to coalescence with other bubbles, respectively. $g\left(V^{\prime}\right)$ represents the breakage rate, which means the rate that a bubble with volume $V$ breaks into bubbles with volume $V^{\prime} \cdot \beta\left(V \mid V^{\prime}\right)$ denotes as probability density function, PDF, of bubbles with volume $V$ breaking to smaller bubbles with volume $V^{\prime} . Q\left(V, V^{\prime}\right)$ is defined as the coalescence rate, at which the bubbles of volume $V$ merge together to produce bubble with volume $V^{\prime}$.

\subsubsection{Breakage model}

The Laakkonen breakage kernel (2005) was used to simulate the breakage frequency and breakage PDF in the aerated coaxial mixer.

$$
\begin{aligned}
& \text { Breakage rate }(\text { kernel })=g\left(V^{\prime}\right) \times \beta\left(V \mid V^{\prime}\right) \\
& g\left(V^{\prime}\right)=2.52 \varepsilon_{l}^{1 / 3} \operatorname{erfc}\left(\sqrt{0.04 \frac{\sigma}{\rho_{l} \varepsilon_{l}^{2 / 3} d_{b}^{5 / 3}}+0.01 \frac{\mu_{l}}{\sqrt{\rho_{l} \rho_{g}} \varepsilon_{l}^{2 / 3} d_{b}^{5 / 3}}}\right)
\end{aligned}
$$

where $\varepsilon, \sigma$, and $\boldsymbol{d}_{\boldsymbol{b}}$ are eddy dissipation of the liquid phase, surface tension, and bubble diameter, respectively. 
The PDF frequency is given as:

$$
\beta\left(V \mid V^{\prime}\right)=\frac{30}{V^{\prime}}\left(\frac{V}{V^{\prime}}\right)^{2}\left(1-\frac{V}{V^{\prime}}\right)^{2}
$$

This model was selected because of the convergence difficulties.

\subsubsection{Coalescence model}

In the stirred vessel, the mechanical energy is dissipated by the impeller to the fluid being mixed, resulting in the creation of the turbulent flow. In this condition, energy cascades from the largest eddies to the smallest one. The size of the smallest eddies is generally given by Kolmogorov microscale, $\eta$, which is formulated based on the turbulent energy dissipation rate and kinematic viscosity as:

$$
\eta=\left(\frac{v^{3}}{\varepsilon}\right)^{1 / 4}
$$

In the turbulent flow, coalescence happens with two different mechanisms including viscous and inertial subrange mechanisms. When the size of the bubbles is less than the Kolmogorov microscale $(\eta)$, the viscous subrange mechanism causes the coalescence of the bubbles, which can be affected by the shear in the eddy. Therefore, according to the Saffman and Turner model (1956), the collision rate is defined as:

$$
a\left(d_{k}, d_{m}\right)=\zeta_{T} \sqrt{\frac{8 \pi}{15}}\left(\frac{\varepsilon}{v}\right)^{1 / 2} \frac{\left(d_{k}+m_{m}\right)^{3}}{8}
$$

here $d_{k}$ and $d_{m}$ are the diameters of bubbles $k$ and $m$, respectively. $\xi_{T}$ takes into account the capture efficiency coefficient of turbulent collision. 
However, when the bubbles are larger than $\eta$, the inertial subrange is applied. In this case, the velocity fluctuations within the fluid flow drag the bubbles. Hence, the coalescence rate is expressed through Abrahamson's model (1975).

$$
a\left(d_{k}, d_{m}\right)=\xi_{T} 2^{2 / 3} \sqrt{\pi} \frac{\left(d_{k}+d_{m}\right)^{2}}{4} \sqrt{U_{k}^{2}+U_{m}^{2}}
$$

where $U_{k}$ is the mean velocity of bubble $k$.

The capture efficiency coefficient is expressed by Higashitani et al. (1983) by the following relation.

$$
\xi_{T}=0.732\left(\frac{5}{N_{T}}\right)^{0.242}
$$

where $N_{T}$ is the ratio between viscous and Van der Waals forces as:

$$
N_{T}=\frac{6 \pi \mu\left(d_{k}+d_{m}\right)^{3} \lambda}{8 H}
$$

In the Eq. (4.1-28) $\boldsymbol{H}$ is Hamaker constant and $\lambda$ is deformation rate.

\subsection{Moving zones approaches}

The modeling of the flow within the agitated vessels is complicated because of the rotation of the impeller. Generally, two approaches have been used in the literature to model the stream generated by the agitators including the steady state multiple reference frame (MRF) and the transient sliding mesh/moving mesh (Fluent tutorial guide Version 6). The aforementioned 
Chapter 4: Model Development

techniques require real agitator geometry and a mesh that includes the stationary and rotating regions.

\subsubsection{Multiple Reference Frame}

MRF is denoted as the steady-state approach, which provides reasonable predictions of the flow in the agitated tank equipped with both stationary and rotating parts. In this approach, one region is created around the impeller, which is considered as the rotating frame, while the remaining region of the vessel is specified as the stationary frame. This approach also can be employed for the vessels equipped with multiple agitators, at which each agitator is modeled with its own rotating frame, whilst the reaming zone is modeled using stationary frame. The stationary and moving frames are joined with a shared surface, at which the transmission of the information takes place constantly as the solution develops. In the simulation, the equations for the stationary and the rotating regions of the domain are computed separately. Using the rotating coordinate systems for the moving zone, the unsteady interactions between rotating and stationary zones are considered.

\subsubsection{Sliding Mesh (SM)}

The sliding mesh is defined as the time-dependent approach in which two grid zones are used similar to the MRF method: one is attached to the agitator, while the other is fixed to the vessel wall. In this approach, moving grid slides relative to the stationary grid. The rotation of the impeller is modeled by the moving grid. The movement of the grid happens in separate steps. In each step the agitator movement is related to its frequency and the specified time step. Luo et al. (1993) was the first one that applied sliding mesh approach to the flow in a stirred tank. Because of the rotating of the coordinate system and the mesh, this method is called transient which makes it 
Chapter 4: Model Development

computationally more complicated than the steady-state approach. It should be noted that although more accurate results can be achieved by SM in comparison with MRF, the sliding mesh approach requires higher calculation time.

\subsection{Modeling of the aerated coaxial vessel}

The CFD simulation of the aerated coaxial system consisting of a four-blade pitched turbine and an anchor is described in this section.

\subsubsection{Geometry and mesh development}

The geometry of the aerated coaxial vessel simulated in the current work was created by Design Modeler (version 16) for the aerated coaxial mixing vessel depicted in Figure 3.1-1. The domain was divided into three zones: two rotating zones, at which the central impeller and anchor located, and one stationary zone (sparger zone), see Figure 4.3-1. These created zones were separated by a common interface to enable the utilization of the sliding mesh (SM) approach, which was employed in this study. Because of the existence of highly complex flow in the aerated coaxial mixing vessel, no symmetry and periodicity was considered.

The developed model was exported to the other pre-processor for discretizing/meshing. The aerated coaxial vessel was three-dimensionally meshed with unstructured tetrahedron elements. Figure 4.3-2 exhibits the mesh constructed for the central impeller; at which local mesh refinement was utilized in order to better capture the turbulent flow details. In the anchor region, the mesh refinement was not as dense as for the central impeller zone, since finer mesh leads to the excessive computation times. Figure 4.3-3 shows the surface mesh for both employed agitators. Also, the inflation process with 7 layers was used on the central impeller and tank wall surfaces to increase the boundary layer resolution (Figure 4.3-4). The quality of the mesh is related to the aspect ratio 
(length and width of a cell), angle and size interval from one cell to another. The developed mesh had the reasonable aspect ratios (ideal ratio is one) in regions near the central impeller blades. In other regions, the gradients are not expected to be high, so that the cells with the higher aspect ratios should not interfere significantly in the quality of the results.

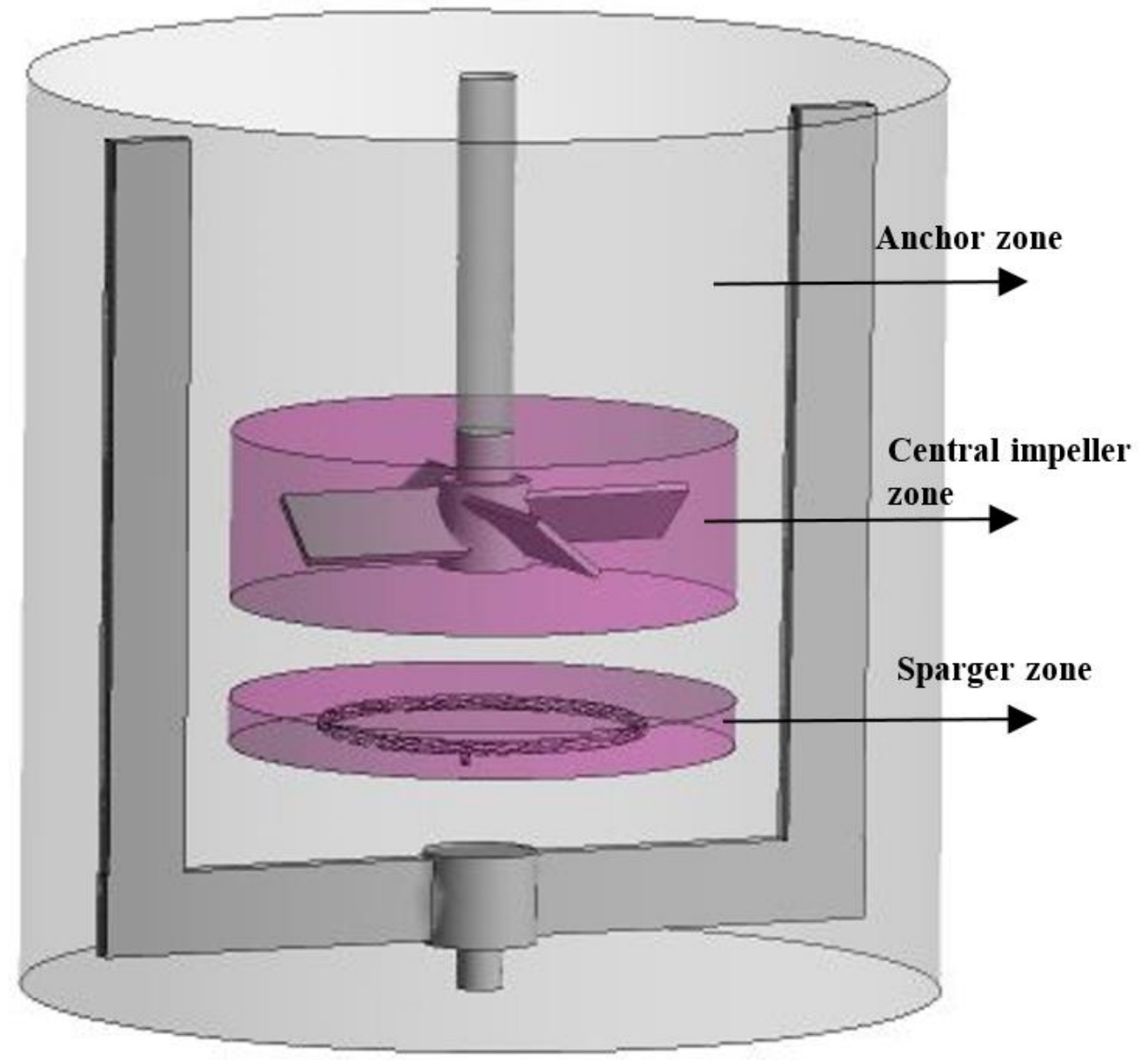

Figure (4.3- 1). Developed model for coaxial vessel using Design Modeller. 


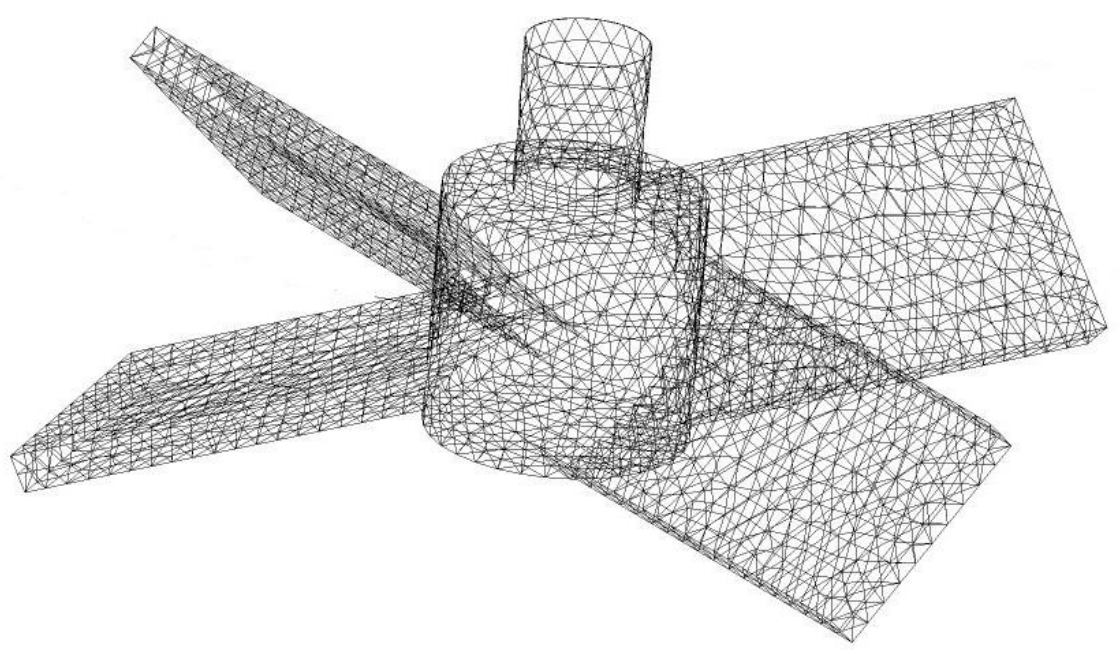

Figure (4.3- 2). Mesh structure for central impeller.

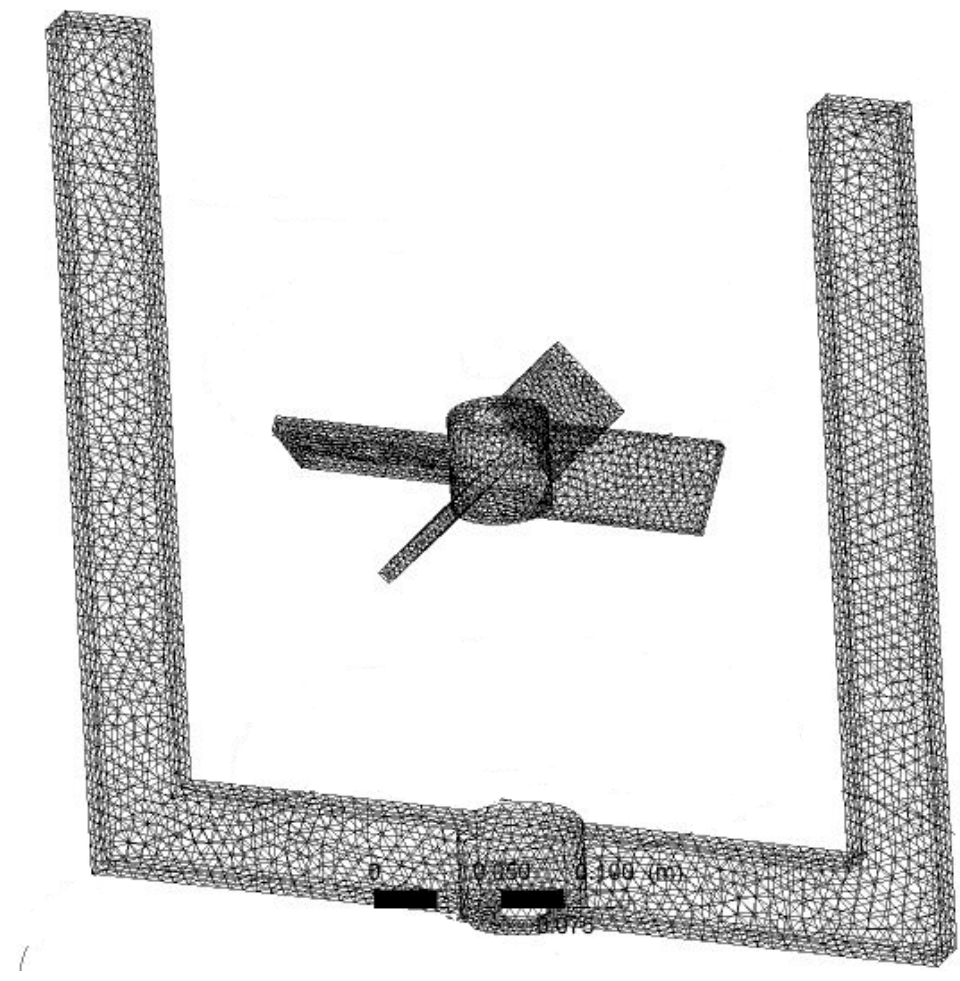

Figure (4.3-3). Surface mesh for central impeller and anchor. 


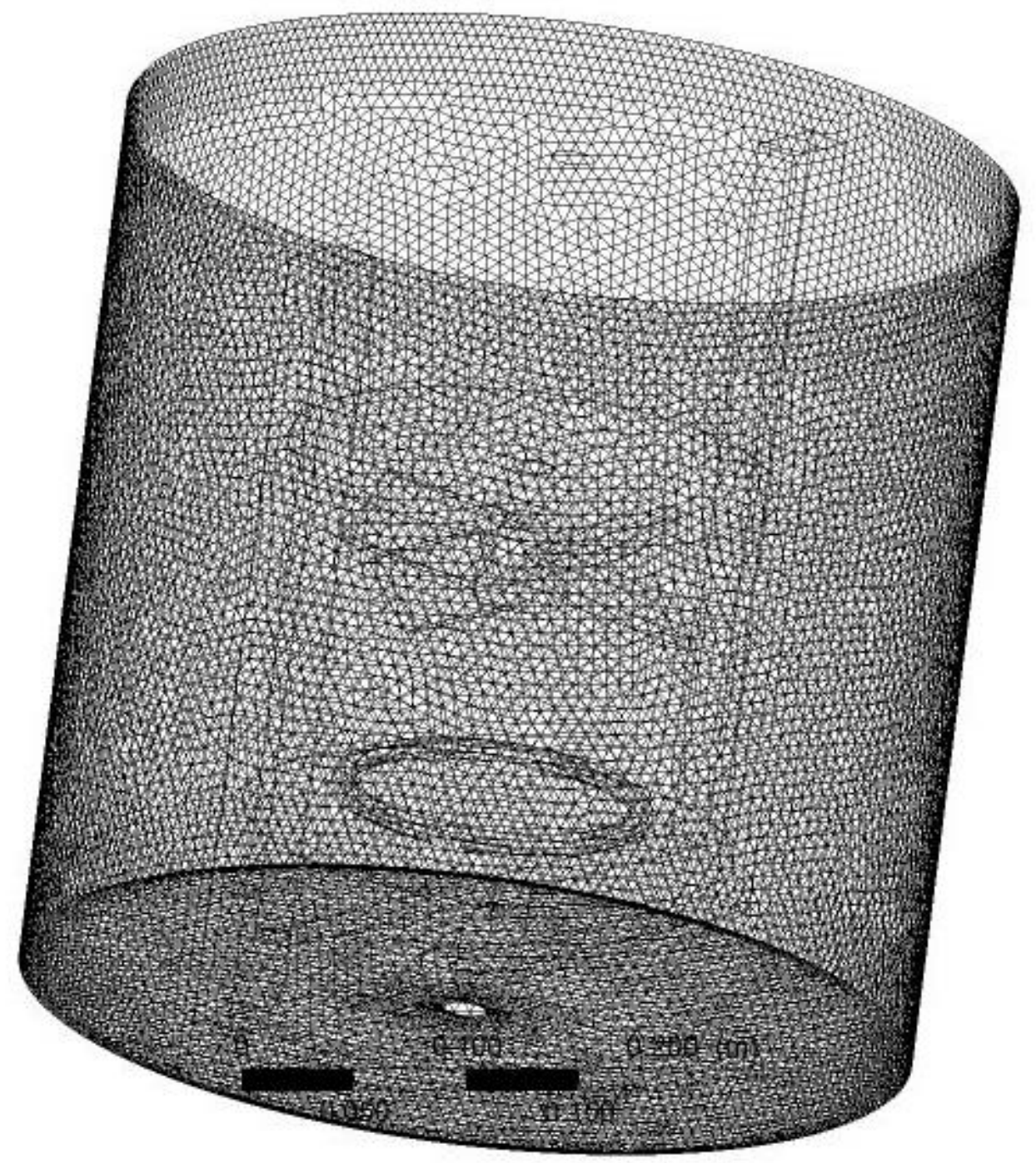

Figure (4.3- 4). Surface mesh developed for the vessel.

\subsubsection{Grid independency and model validation}

The accuracy of the CFD results depends on the quality of the grids. Although the higher number of cells enhances the accuracy of the results, it increases the computational time. Therefore, a grid 


\section{Chapter 4: Model Development}

independence study was conducted to obtain the optimized number of cells, at which the error reduction was insignificant and the acquired results became independent of the mesh size. To achieve this goal, three different grid systems including coarse (920954), medium (1921386), and fine (2969469) were employed. The simulations were performed at the central speed of $1600 \mathrm{rpm}$, the anchor speed of $133 \mathrm{rpm}$, and the aeration rate of $0.0283 \mathrm{~m}^{3} / \mathrm{min}$. Since the parameters of interest in this work were the gas holdup and developed velocity profiles in the aerated coaxial mixing vessel, these parameters were selected for the grid independence test. Two lines in the high-velocity-gradient regions of the aerated coaxial mixer, near the central impeller and anchor, were specified, see Figure 4.3-5. Along the aforementioned lines, the radial, axial, and tangential velocities of syrup were compared for three different meshes in Figure 4.3-6 and Figure 4.3-7. Besides, in Figure 4.3-8 gas holdup contours of three mesh systems were depicted. As it can be seen, the obtained results in case 1 (coarse mesh) was far from the others cases, therefore it was rejected. To be illustrated, by increasing the number of cells from coarse to medium, the velocity magnitude of the corn syrup in the high-velocity-gradient regions varied by more than $6.0 \%$. However, by further increase in the number of cells, from medium to fine, the difference between the velocity magnitudes in the region of high velocity gradients were insignificant (maximum deviation was less than $2 \%$ ). Hence, between the medium and the fine grids, the medium one was selected for all simulations because of being less computational demanding. 


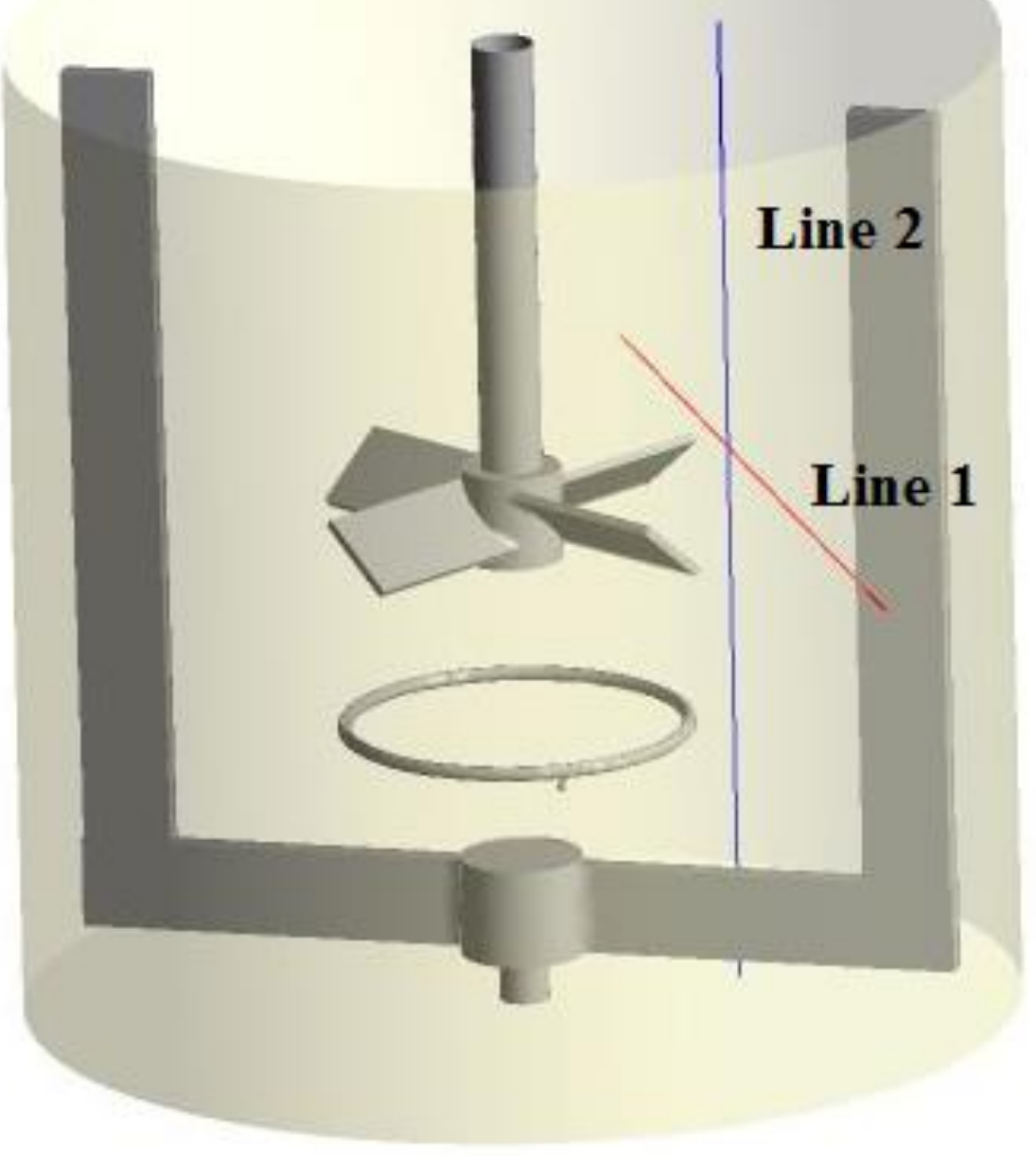

Figure (4.3- 5). Specified lines for grid independence study. 


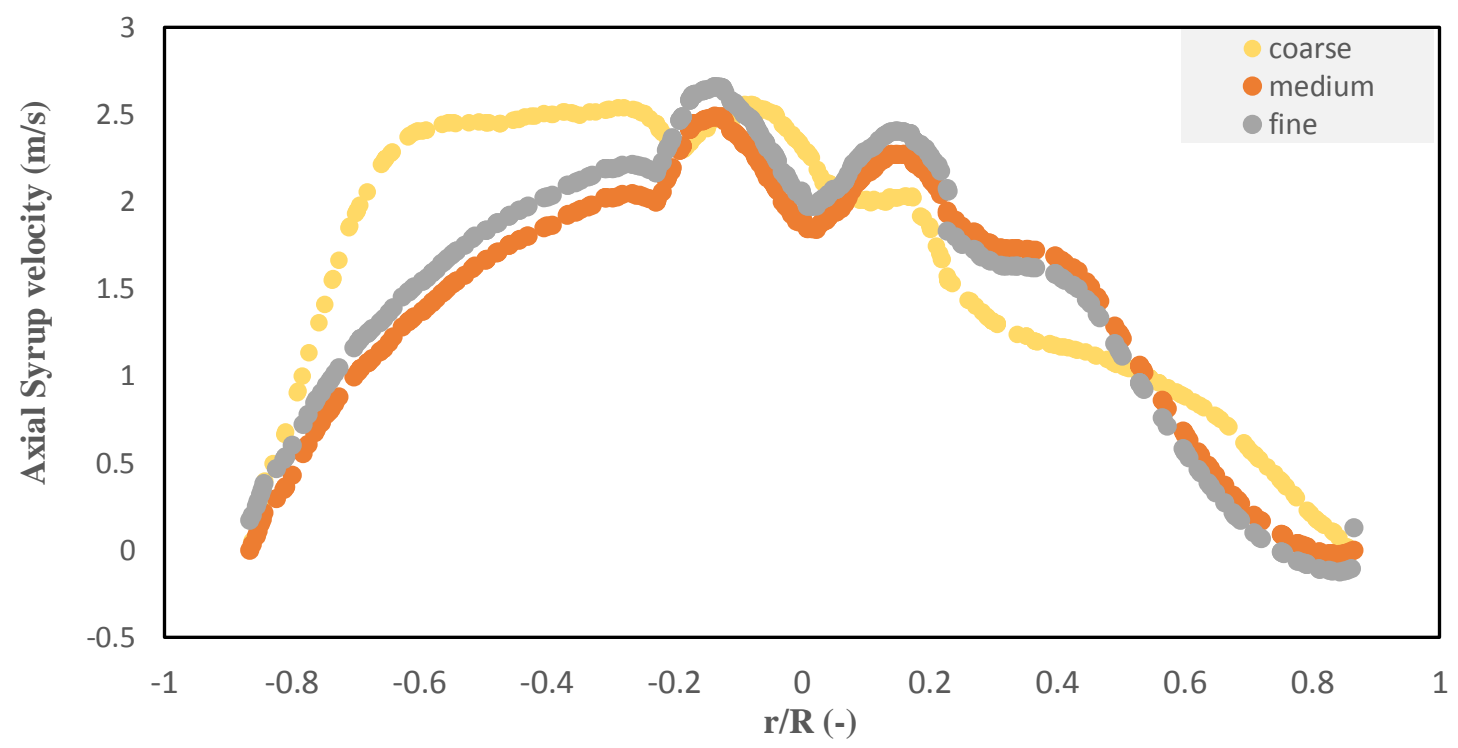

(a)

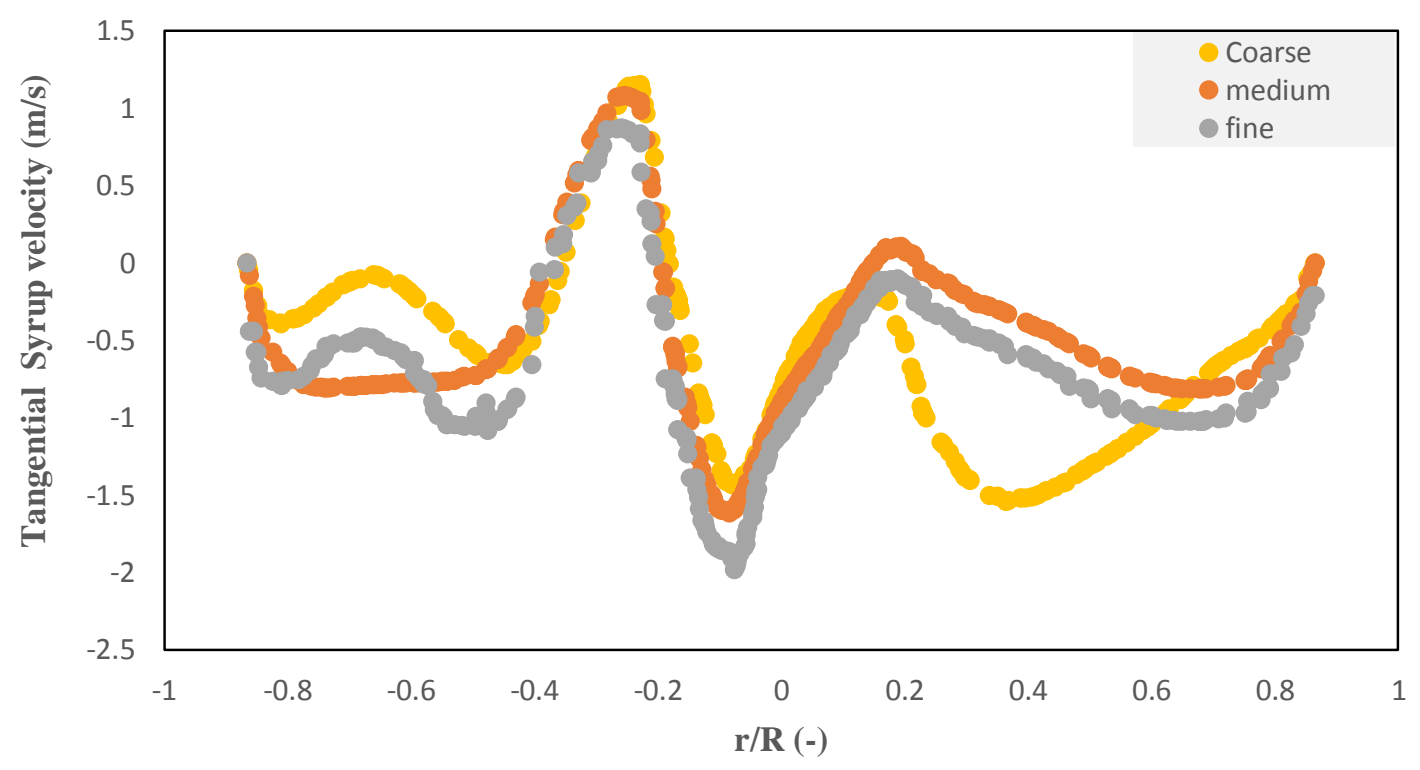

(b)

Figure (4.3- 6). Effect of the number of grids on the (a) axial velocity and (b) tangential velocity in horizontal position close to the central impeller [Line 1 in Figure (4.3-5)]. 


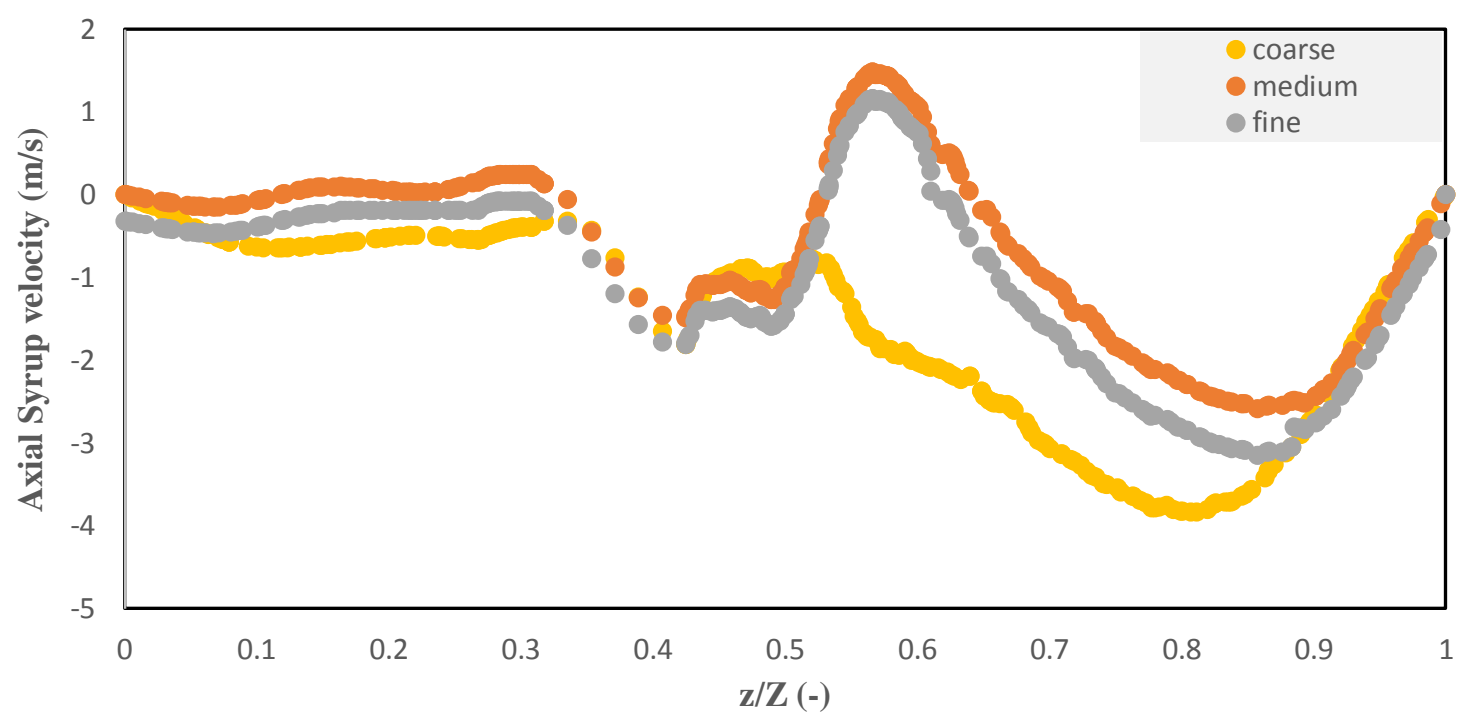

(a)

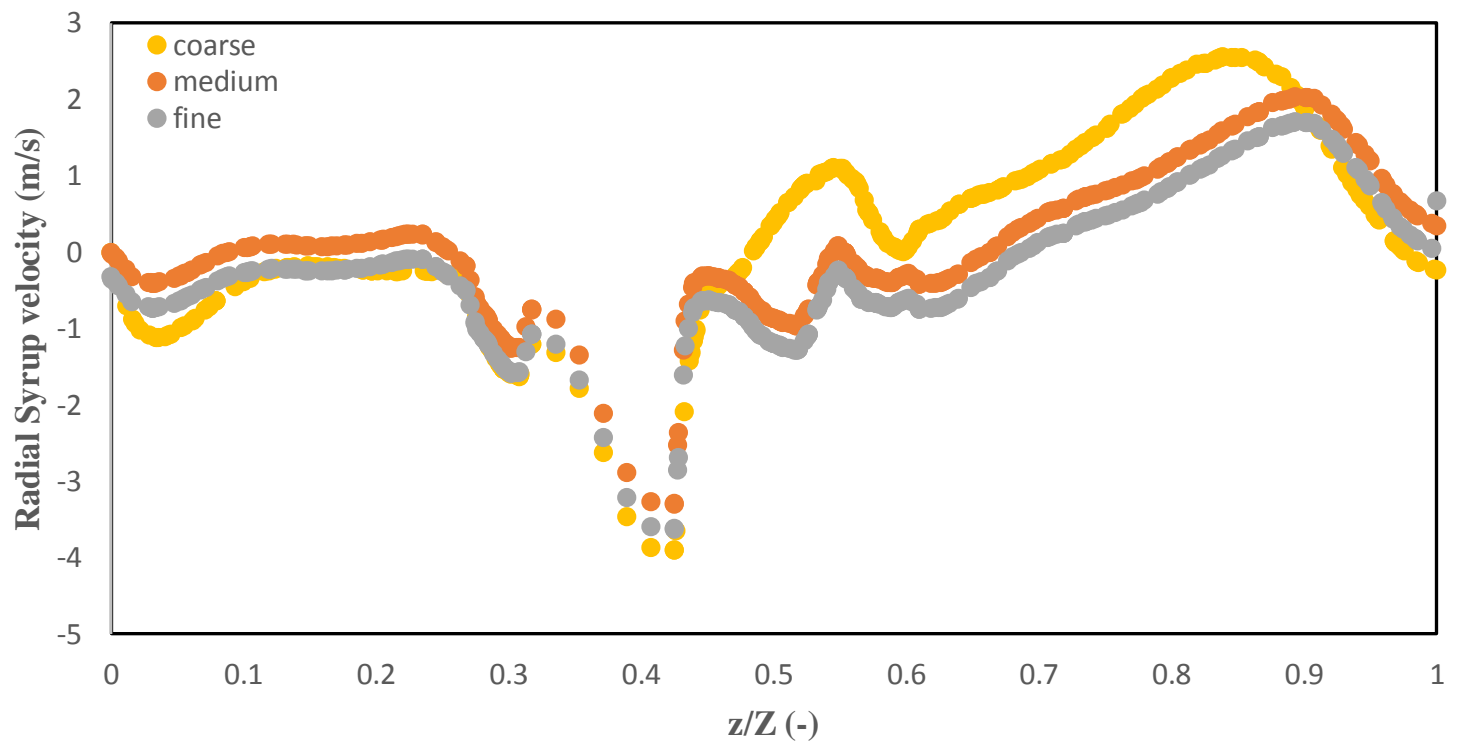

(b)

Figure (4.3- 7). Effect of the number of grids on the (a) tangential velocity, and (b) radial velocity in vertical position close to anchor impeller [Line 2 in Figure (4.3-5)] 

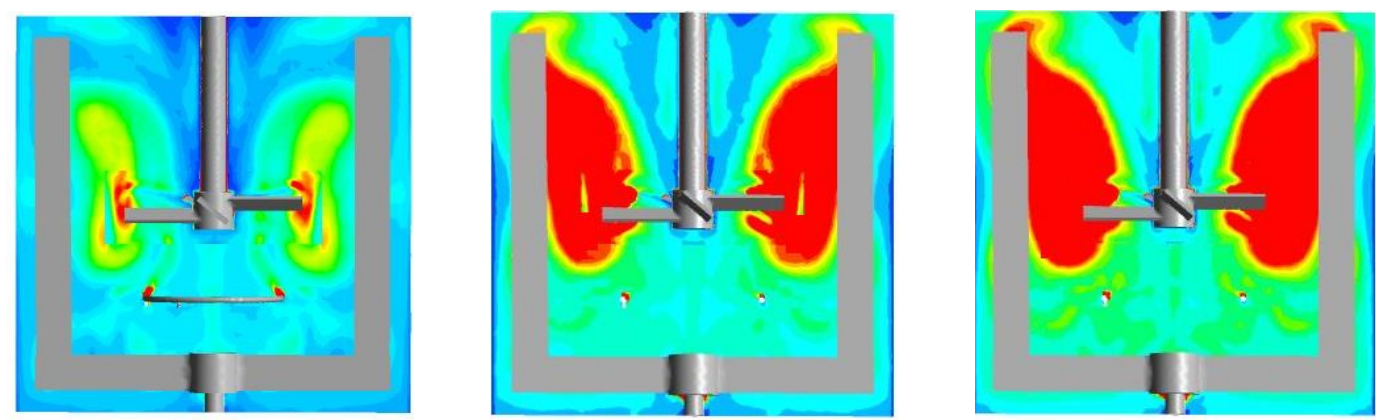

$9.000 \mathrm{e}-002$

8.100e-002

$7.200 \mathrm{e}-002$

$6.300 \mathrm{e}-002$

$5.400 \mathrm{e}-002$

$4.500 \mathrm{e}-002$

$3.600 \mathrm{e}-002$

$2.700 \mathrm{e}-002$

$1.800 \mathrm{e}-002$

$9.000 \mathrm{e}-003$

$0.000 \mathrm{e}+000$

Coarse

Fine

Figure (4.3- 8). Effect of different grid sizes on the gas holdup distribution.

To validate the developed CFD model in the current study, the predicted values of power consumption and gas holdup were compared to our experimental data. The experimental measurement techniques of power uptake and gas holdup have been described in Experimental Setup and Procedure (sections 3.2.1-2, 3.2.3). Table 4.3-1 shows the experimental and simulation results at different operating conditions. It should be noted that these results obtained when single bubble size assumption was adopted. As it can be seen, the values of total gas holdup were overpredicted by the CFD model. The possible reasons can be related to the assumption of a single bubble size within the vessel, and insufficiencies of the bubble drag force. Overall, the CFD model reasonably predicted the gas-liquid flow in the aerated coaxial mixing vessel. In the case of population balance model coupled with CFD, the validation was conducted using Sauter mean bubble diameters at different points inside of the vessel, see section 5.5.2.1. These outcomes demonstrated that the predicted Sauter mean bubble diameters were in fairly good agreement with the experimental ones. 
Table (4.3- 1). Validation of simulation results with experimental data.

\begin{tabular}{ccccccc}
\cline { 4 - 6 } & & & Total Gas holdup (\%) & Total power (W) \\
\hline $\begin{array}{c}\text { Central impeller speed } \\
(\mathbf{r p m})\end{array}$ & $\begin{array}{c}\text { Anchor speed } \\
(\mathbf{r p m})\end{array}$ & $\begin{array}{c}\text { Gas Flow rate } \\
\left(\mathbf{m}^{3} / \mathbf{m i n}\right)\end{array}$ & Experiment & Simulation & Experiment & Simulation \\
\hline 180 & 18 & 0.0283 & 5.10 & 6.07 & 6.50 & 6.44 \\
\hline 180 & 15 & 0.0283 & 5.70 & 6.44 & 6.12 & 5.92 \\
\hline 150 & 15 & 0.0189 & 3.40 & 4.35 & 4.54 & 4.43 \\
\hline 150 & 12.5 & 0.0189 & 3.60 & 3.99 & 4.20 & 4.17 \\
\hline
\end{tabular}

\subsection{Comparison of different drag models}

Three different drag models with respect to gas holdup distribution within the aerated coaxial mixing vessel were compared. The considered models were Grace drag model, Ishii-Zuber drag model and modified Tomiyama model based on Brucato model. Table 4.4-1 shows the comparison of averaged gas holdup values with three drag models. All the simulations were conducted at the same operating conditions: $N_{c}=180 \mathrm{rpm}, N_{a}=15 \mathrm{rpm}$, and $Q_{g}=0.0283 \mathrm{~m}^{3} / \mathrm{min}$.

As it can be seen in Table 4.4-1, the modified Tomiyama model and grace model resulted in the highest and the lowest gas holdups, respectively. Also, the outcome of the modified Tomiyama model was closer to the obtained experimental data. Therefore, in the current study the modified Tomiyama model based on Brucato model was used for all the conducted simulations.

Table (4.4- 1). Comparison of different drag models.

\begin{tabular}{lcccc}
\hline & Grace model & Ishii-Zuber & Modified Tomiyama & Experimental \\
\hline Averaged gas holdup & 0.033 & 0.041 & 0.064 & 0.057 \\
\hline
\end{tabular}




\subsection{Time step independency}

In addition to the grid size, time step is an important parameter which can affect the accuracy of the solution. Therefore, an optimum time step should be determined to achieve the convergence and to obtain the accurate results. To ascertain time step independence, four different time steps corresponding to $0.5,1,5$, and 10 degrees of rotational angle of the central impeller have been used. In order to check the sensitivity of the time step on the accuracy of the simulation results, the effect of time step on the simulated averaged gas holdup throughout the aerated coaxial mixer were examined. The predicted gas holdup values by 0.5 and 1 degrees of the central impeller rotation resulted in a small difference, less than $1.7 \%$ deviation. Therefore, the time step was selected as a time to rotate the central impeller one degree, which led to less computational demand.

\subsection{Simulation characteristics}

As mentioned before, a CFD package (ANSYS-Fluent Version 16) was employed to simulate the developed hydrodynamics within the aerated coaxial mixing vessel. Rotations of the central impeller and the anchor were modeled with sliding mesh approach. The vessel walls, impellers, and sparger were treated as non-slip boundary conditions. The boundary condition for the top surface of the sparger was set as the velocity inlet. Afterwards, the appropriate gas velocity and the gas volume fraction equal to unity were considered at the top surface of the ring sparger to introduce the gas phase into the vessel. The top surface of the liquid was modeled as the degassing boundary condition, which allowed the gas bubbles to escape into atmosphere while the liquid 
phase saw this boundary as a free slip wall and did not leave the domain. The PBM model was solved using MUSIG method with fifteen bubble bins ranging from 0.5 to $12 \mathrm{~mm}$. The SIMPLE algorithm was utilized to couple the pressure and the velocity. The Second Order upwind, QUICK, and power law discretization schemes were utilized for momentum, gas volume fraction, and gas bins, respectively. It should be mentioned that when population balance was added to the developed model, a higher order term relaxation, and a bounded second order implicit transient formulation were employed to enhance the convergence stability. In section 5.4, since the experimental data of bubble size distributions in the aerated coaxial mixer was not available, single bubble size approach was adopted. The basis for the selection of the considered bubble size was based on the correlation proposed by Kumar et al. (1976). The estimated bubble size was almost $7 \mathrm{~mm}$ for all considered speed ratios; therefore the same bubble size $(7 \mathrm{~mm})$ was utilized to simulate the gas-liquid behavior in the aerated coaxial mixer. The solutions were considered to be converged when the normalized residuals of all the variables were below $10^{-6}$. The simulations were run in parallel with 12 dual cores SUN Ultra-Spark IV, 1.8 GHz Sun Micro-Systems CPUs applying computing facilities of HPCVL (High Performance Computing Virtual Laboratory). The computational time for most of the simulations was approximately 111 hours. 


\section{Chapter 5}

\section{Results and Discussions}

In the current work, the mixing performance of a novel aerated coaxial mixing vessel consists of a central impeller and an anchor was investigated. To fulfill this objective, the experimental and numerical studies were conducted. The characterization was performed in regard to mixing time, power uptake, local and global gas holdup values, and bubble size distribution throughout the aerated coaxial mixer. In this chapter all the results and related discussions are presented in the following five subsections as:

\section{$>\quad$ 5.1. Analysis of mixing in an aerated reactor equipped with the coaxial mixer through electrical resistance tomography and response surface method}

Mixing characteristics of an aerated coaxial mixer composed of an anchor and a central impeller was investigated using the non-invasive flow visualization technique called electrical resistance tomography (ERT). Corn syrup solutions with different viscosity were used as the viscous Newtonian fluids. Two coaxial configurations were considered: the anchor - PBD (a pitched blade downward pumping impeller) and the anchor - PBU (a pitched blade upward pumping impeller). In this section (5.1), the effects of central impeller types, speed ratios (central impeller speed/anchor speed), rotation modes, gas flow rates, and viscosity on the mixing time and power

uptake were explored. It was found that in the presence of gas, the PBU-anchor coaxial 
Chapter 5: Results and Discussion

combination in co-rotating mode exhibited shorter mixing times and lower power consumption than the PBD-anchor. Using the response surface method, an effort was made to develop a quadratic model as a function of central impeller speed, anchor speed, gas flow rate, and viscosity for predicting the mixing time. Three-dimensional response surfaces were plotted to understand the main and interaction effects of these factors. The outcomes of this section were published in Chemical Engineering Research and Design Journal (Hashemi et al., 2016a).

$>\quad$ 5.2. Analysis of power consumption and gas holdup distribution for an aerated reactor equipped with a coaxial mixer: Novel correlations for the gas flow number and gassed power

The main objectives of section 5.2 were to study the gassed power consumption and the local gas holdups for an aerated coaxial mixer equipped with a wall scraping anchor and a central impeller. The working fluids were viscous corn syrup solutions at different concentrations. The central impellers were downward pumping pitched blade turbine, upward pumping pitched blade turbine, and hydrofoil A310 impellers. A novel correlation was developed to estimate the gassed power drawn by the coaxial mixer as a function of the gas flow rate, central impeller type, rotational speeds of the anchor and central impeller, and the ungassed power consumption by the anchor and the central impeller. To obtain a master power curve, two new correlations were proposed for the generalized power number and gas flow number of the aerated reactor furnished with the coaxial mixer by incorporating the equivalent rotational speed for the coaxial mixer, speed, and the central impeller power fraction into these two correlations. To measure the distribution of the gas holdup throughout the aerated reactor, four electrical resistance tomography planes were installed around the reactor. The radial gas holdup values were assessed. The obtained results in this section have been published in Chemical Engineering Science (Hashemi et al., 2016b). 
Chapter 5: Results and Discussion

\section{$>\quad$ 5.3. Experimental investigation of the bubble behavior in an aerated coaxial mixing vessel through electrical resistance tomography (ERT)}

To enhance the efficiency of the aerated mixing vessel, it is critical to investigate the influence of the flow hydrodynamics generated by the impeller on the bubble size and gas holdup. In the current section, the global and local gas holdup values and the Sauter mean bubble diameter for an aerated coaxial mixing vessel equipped with a central pitched blade turbine and an anchor impeller were estimated for the viscous corn syrup solution. The main characteristics of the bubbles including the number of bubble size classes and the contribution of each class of bubble in the overall gas holdup were measured by using the dynamic gas disengagement theory coupled with the electrical resistance tomography (ERT) data. The impacts of the speed ratio and the gas flow rate on the bubble behavior (size and dispersion) were also explored. In addition, the effect of the speed ratio on the bubble breakup through anchor blades was demonstrated. Gas holdup was correlated to the speed ratio, aerated power per liquid volume, and gas velocity. The acquired results were published in Chemical Engineering Journal (Hashemi et al., 2016c).

\section{$>\quad$ 5.4. Hydrodynamic characteristics of an aerated coaxial mixing vessel equipped with a pitched blade turbine and an anchor using CFD}

Computational fluid dynamic (CFD) is a significant tool for conducting the realistic simulations of the process equipment. In section 5.4, CFD simulation of the aerated coaxial mixing vessel was conducted to investigate the effects of speed ratio, rotation modes (co-rotating and counterrotating), and fluid viscosity on the local and global gas holdup values, flow pattern within the vessel, and turbulent kinetic energy. All simulations were carried out using the sliding mesh approach to model the rotation of both impellers. Eulerian-Eulerian two-fluid model with the RNG k-epsilon turbulence model were employed. To validate the developed model, simulated gas 
Chapter 5: Results and Discussion

holdup and gassed power uptake were compared with the measured experimental values. To gather experimental gas holdup values, electrical resistance tomography technique (ERT) was utilized. The outcomes of this section have been submitted to the Journal of Chemical Technology and Biotechnology (Hashemi et al., 2017).

\subsection{Modeling of bubble size distribution in a bioreactor equipped with a coaxial mixer using the population balance model coupled with CFD}

In this work, the effect of the speed ratio on the bubble size distributions, BSD, in an aerated coaxial mixer was investigated using computational fluid dynamics (CFD) coupled with population balance method. The population balance for bubbles was determined using the discrete method, in which the influence of bubble breakup and coalescence on the BSD was considered. In this study, bubbles were divided into fifteen bins ranging from $0.5 \mathrm{~mm}$ to $12 \mathrm{~mm}$ (observed in experiments under the same operating conditions). To validate the model, the predicted and measured Sauter mean bubble diameters in the various points inside of the coaxial mixer were compared and good agreement was achieved. The obtained results showed that the gas dispersion was enhanced at the speed ratios higher than 10 due to the reduction in the volume fractions of the large bubbles. The aforementioned phenomenon was more significant at the speed ratio of 12 . 
Chapter 5: Results and Discussion

\subsection{Analysis of mixing in an aerated reactor equipped with the coaxial mixer through electrical resistance tomography and response surface method}

\subsubsection{Introduction}

Several factors such as level of homogeneity and power consumption must be considered in designing an efficient mixing system. Using mixing time, useful information can be obtained about the flow inside the vessel. The shorter the mixing time, the more efficient the blending becomes. In the absence of gas, the data demonstrated that the coaxial systems operating in the co-rotating mode exhibited a better performance in comparison to the ones in the counter-rotating mode (Pakzad et al., 2013a, 2013b, 2013,c; Bao et al., 2011; Rudolph et al., 2007; Foucault et al., 2006). However, no data have been reported on the mixing time of the aerated coaxial systems.

For the single-shaft aerated mixing vessels, mixing time as a function of the power drawn has been studied by many researchers (Van’t Riet, 1976; Nienow \& Wisdom, 1974). Nonetheless, much less research has been carried out to define the effects of aeration on the mixing time and it is still an open question. Some studies demonstrated that the aeration decreases the mixing time (Vasconcelos et al., 1995), while others (Machon \& Jahoda, 2000) indicated that mixing time can be enhanced and reduced by gassing, according to the dominance of gas flow rate or impeller rotational speed. The gas-liquid flow pattern plays a crucial role in the aerated vessels. Therefore, all the gas-liquid flow conditions such as flooding, loading, and complete dispersion should be considered to study the impact of gassing on the mixing time and power consumption of the aerated coaxial mixers.

The goal of the present work is to analyze the mixing time and power consumption of an aerated coaxial mixer used for the agitation of the corn syrup, a Newtonian fluid. Electrical resistance 
Chapter 5: Results and Discussion

tomography (ERT) was used to measure the mixing time in this work. Two combinations of central impeller, namely upward pumping pitched blades-anchor and downward pumping pitched bladesanchor were considered. Besides, the effect of the speed ratios, operating modes (co-rotating and counter-rotating), gas flow rates, and viscosity were investigated. Recognition of the most efficacious factors and possible interaction effects between them on the mixing time in an aerated coaxial system are challenging tasks. The response surface methodology (RSM) has been proven as a valid statistical tool to overcome these difficulties. This method can provide the optimal response by considering the interactions among variables with minimum number of experiments (Silva et al., 2007). Using four-factor three-level Box-Behnken combined with RSM, a quadratic model was established in terms of mixing time as a function of the central impeller speed, anchor speed, gas flow rate, and viscosity.

It should be mentioned that the subsequent dimensionless numbers (Reynolds, power and flow numbers) were used in this study (Foucault et al., 2005):

$$
\begin{aligned}
& \operatorname{Re}=\frac{\left(N_{c} \pm \frac{N_{a}}{R_{N}}\right) D_{c}^{2} \rho}{\mu} \\
& N_{p}=\frac{P_{g t o t}}{\rho\left(N_{c} \pm \frac{N_{a}}{R_{N}}\right)^{3} D_{c}^{5}} \\
& F l_{g}=\frac{Q_{g}}{N_{c} D_{c}^{3}}
\end{aligned}
$$


Chapter 5: Results and Discussion

\subsubsection{Results and Discussions}

\subsubsection{Single impellers/coaxial mixers}

Several studies have been conducted to compare the performance of the coaxial mixers to the single impellers in terms of mixing time for the single phase liquids. It has been concluded that the coaxial mixers in the co-rotating mode gives the shortest mixing times, followed by the counterrotating coaxial mixers and the single impeller mixer with the stationary anchor, respectively (Pakzad et al., 2013a, 2013b; Foucault et al., 2004, 2005, 2006; Espinosa-Solares et al., 2002). The reason is that the central impeller and wall scraping anchor can support each other in the co-rotating mode to strengthen the fluid mixing throughout the tank. As an illustration of the superior performance of coaxial mixers, especially in the co-rotating mode over single impeller systems, Figure 5.1-1 depicts the variation of mixing time versus Reynolds number for the corn syrup solution in the absence of aeration. Same trends were obtained for all considered mixing systems and as it can be seen by increasing the Reynolds number mixing time was decreased. The mixing times for the coaxial systems were shorter than the corresponding times for any of the single impeller configurations at all Reynolds number. These results also show that the coaxial systems equipped with PBD shortened the mixing times in comparison with the ones comprised of PBU in the absence of gas. The acquired outcomes match with those published in the literature (Kumaresan \& Joshi, 2006; Patwardhan \& Joshi, 1999). Their explanation was attributed to the fact that the flow leaving the PBD interacts with the vessel bottom and therefore stronger dissipation energy is formed beneath the agitator. Nonetheless, the flow generated by PBU interacts with the liquid surface and produces lower dissipation energy which results in higher mixing times. It should be mentioned that PBD and PBU show diverse behaviors in the gassing conditions which is explained in the following sections. 


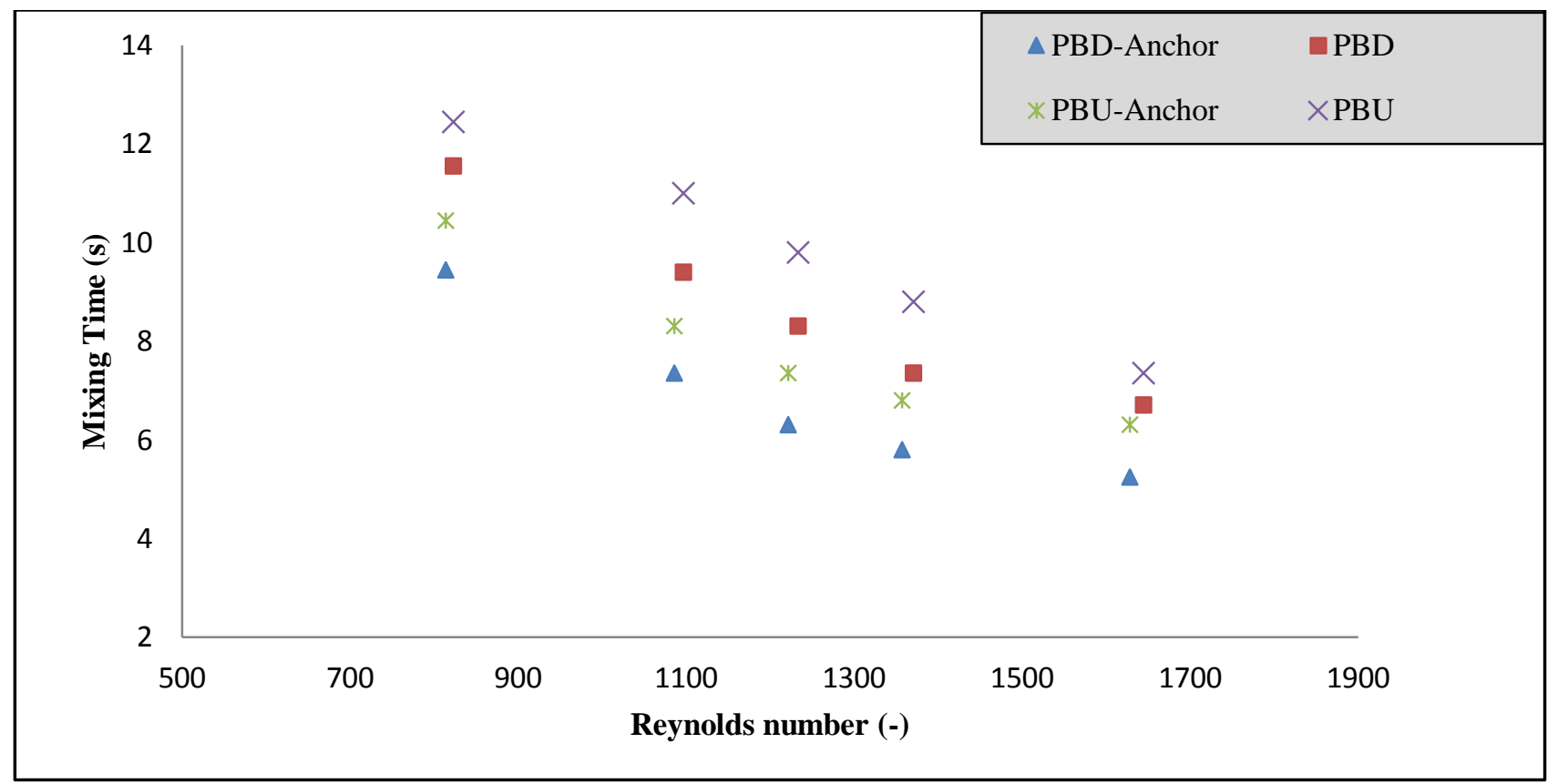

Figure (5.1- 1). Mixing time versus Reynolds number for the coaxial mixers in the co-rotating mode and single impellers s (max $\mathrm{STDEV} \approx 1.8 \%)$.

\subsubsection{Effect of the central impeller speed}

The mixing time as a function of Reynolds number for the PBD-anchor and PBU-anchor coaxial systems under gassed conditions is presented in Figure 5.1-2a-c. It was observed that at low Reynolds numbers $(\operatorname{Re}<1100)$, corresponding to flooding regime, increasing the central impeller speed at constant gas flow rate raised the mixing time. The highest mixing time value of each profile corresponds to the onset of dispersion. Furthermore, it was concluded that the Reynolds number of the onset of dispersion was irrespective of the rotating modes of anchor and central impeller, central impeller pumping modes (upward or downward), and anchor speeds. By further increasing the Reynolds number, loading/dispersion happened and increasing the central impeller speed at constant gas flow rate resulted in the reduction of the mixing time. In addition, comparing the data obtained for the PBD-anchor and the PBU-anchor revealed that the performance of the coaxial system comprising PBU in co-rotating mode was superior in the presence of gas. Other 
Chapter 5: Results and Discussion

studies have indicated that PBU impellers outweigh PBD impellers in the aerated mixing systems (Machon \& Jahoda, 2000). They reported that in an aerated system and at constant gas flow rate, the exchange liquid flows for the system equipped with the pitched blade upward pumping impeller was higher than that achieved for the pitched blade downward pumping impeller. Aubin et al. (2001) also came to this conclusion that by utilizing downward pumping axial impellers in the agitated tanks, only a single flow loop was generated near the impeller and therefore at upper part of the tank poor mixed regions were observed. However, upward pumping impellers provided two flow loops throughout the tank, resulted in the better mixing. Mixing time can be affected by the speed ratio as illustrated in Figure 5.1-3a and b. As it can be seen, the coaxial mixers with anchor rotating at $10 \mathrm{rpm}$ showed the lowest mixing times. The reason is that, at high speed ratio (low anchor speed), the flow pattern is mainly governed by the central impeller which has the main role for gas dispersion and homogenization. However, at low speed ratios, tangential flow pattern generated by anchor is dominated, leading to an increase in the mixing time. 
Chapter 5: Results and Discussion

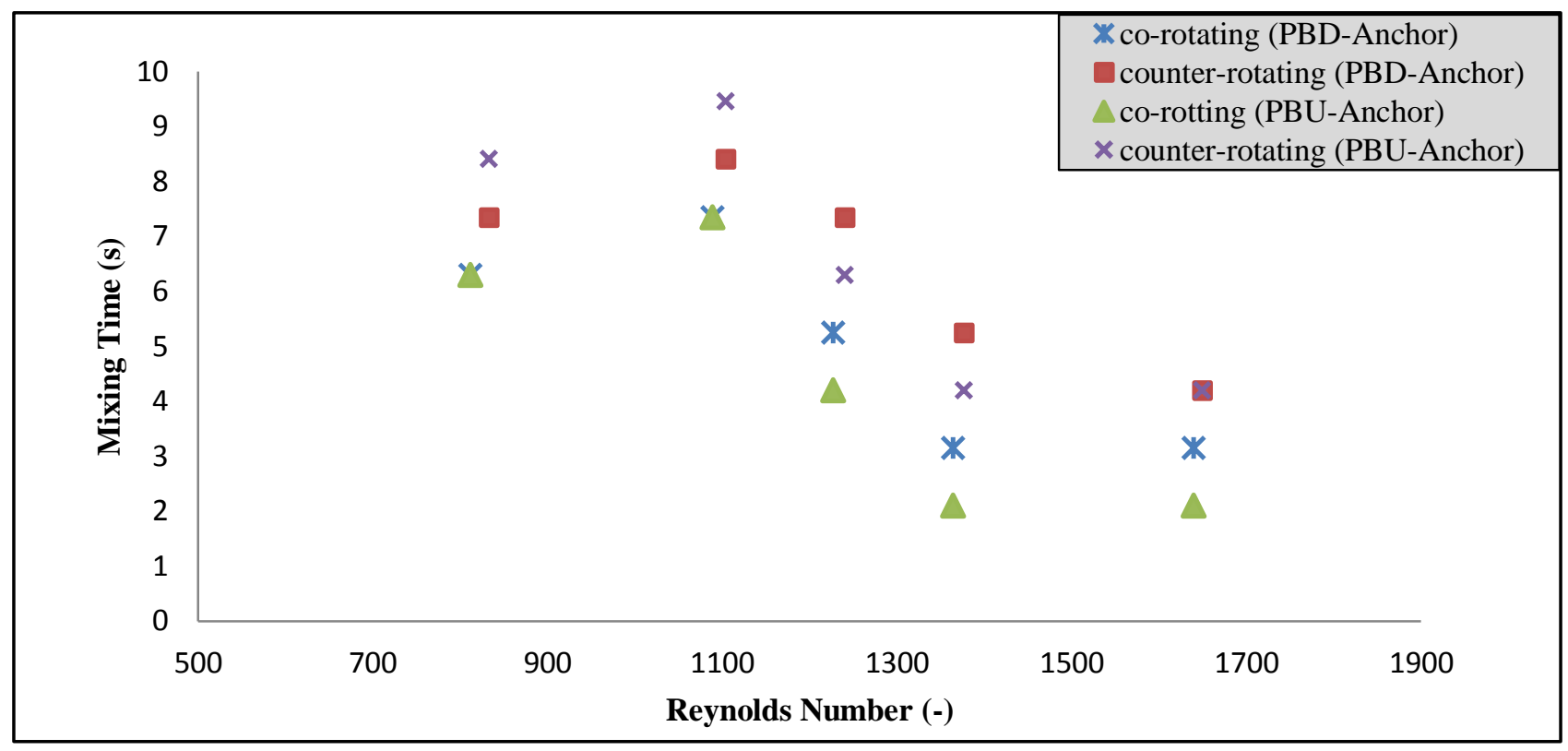

(a)

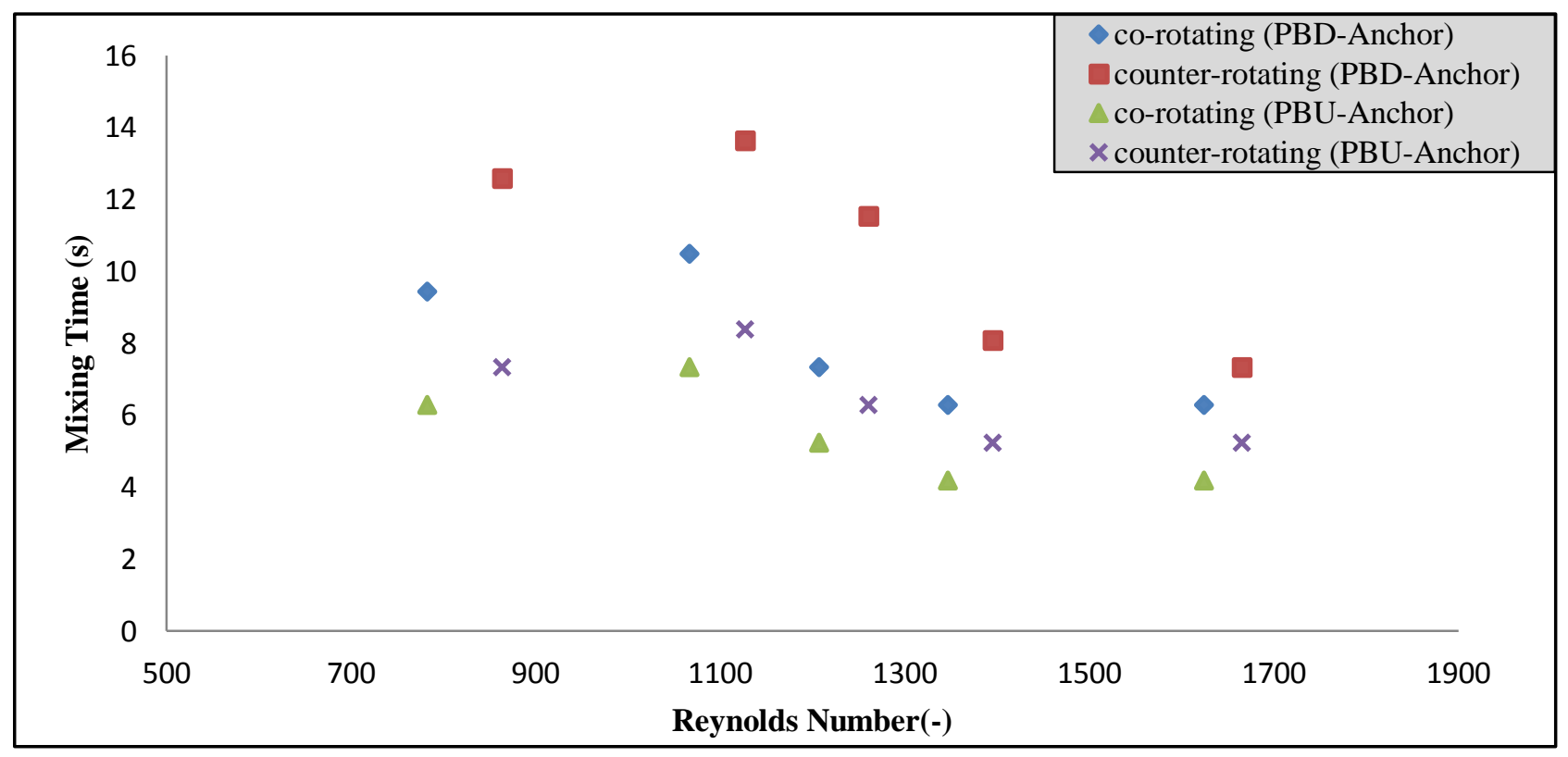

(b) 
Chapter 5: Results and Discussion

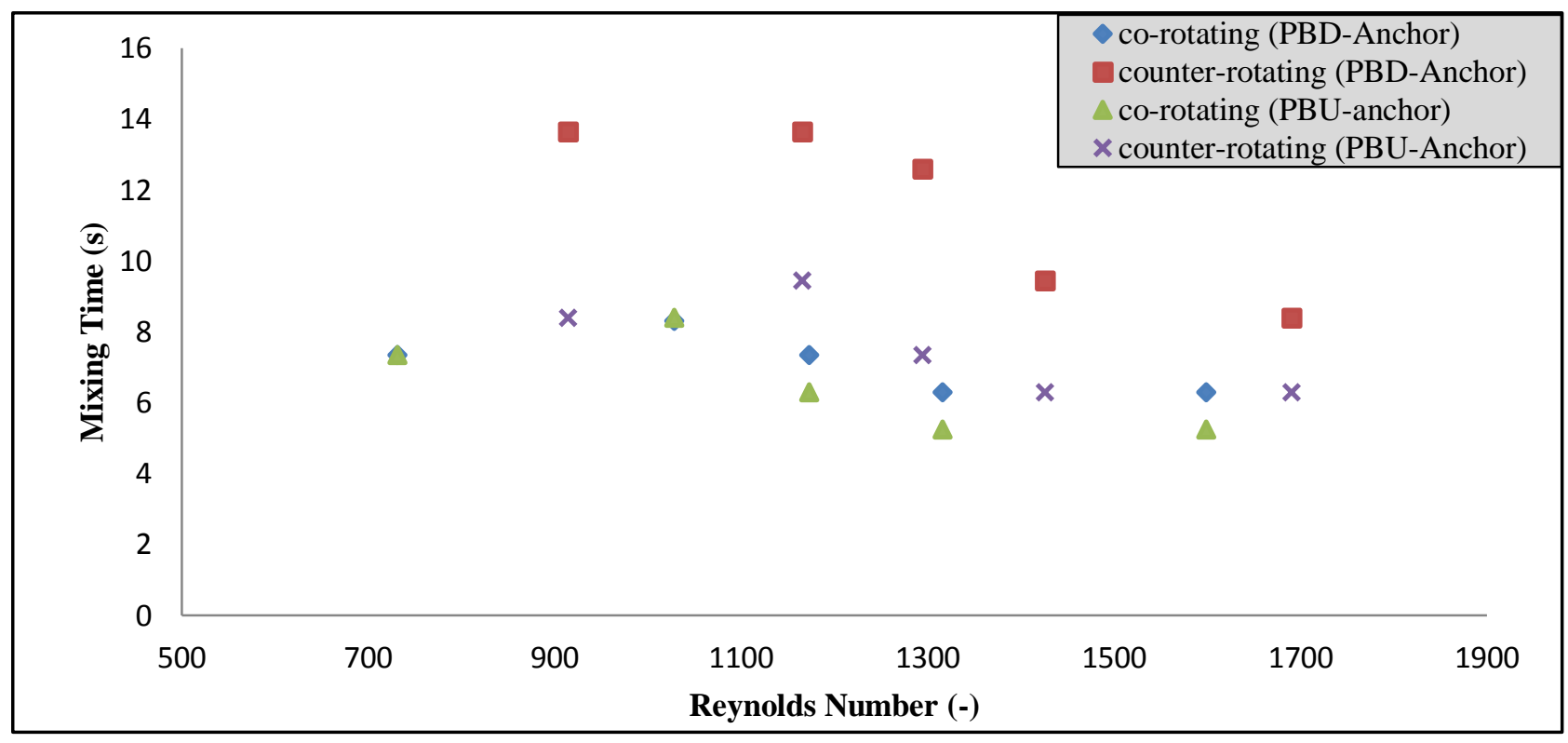

(c)

Figure (5.1 - 2). Mixing time versus Reynolds number when only the central impeller speed changes at constant gas flow rate of $0.0283 \mathrm{~m}^{3} / \mathrm{min}$ and the anchor speed of (a) $10 \mathrm{rpm}$, (b) $20 \mathrm{rpm}$, and (c) $30 \mathrm{rpm}$ (max $\mathrm{STDEV} \approx 1.69 \%$ ). 
Chapter 5: Results and Discussion

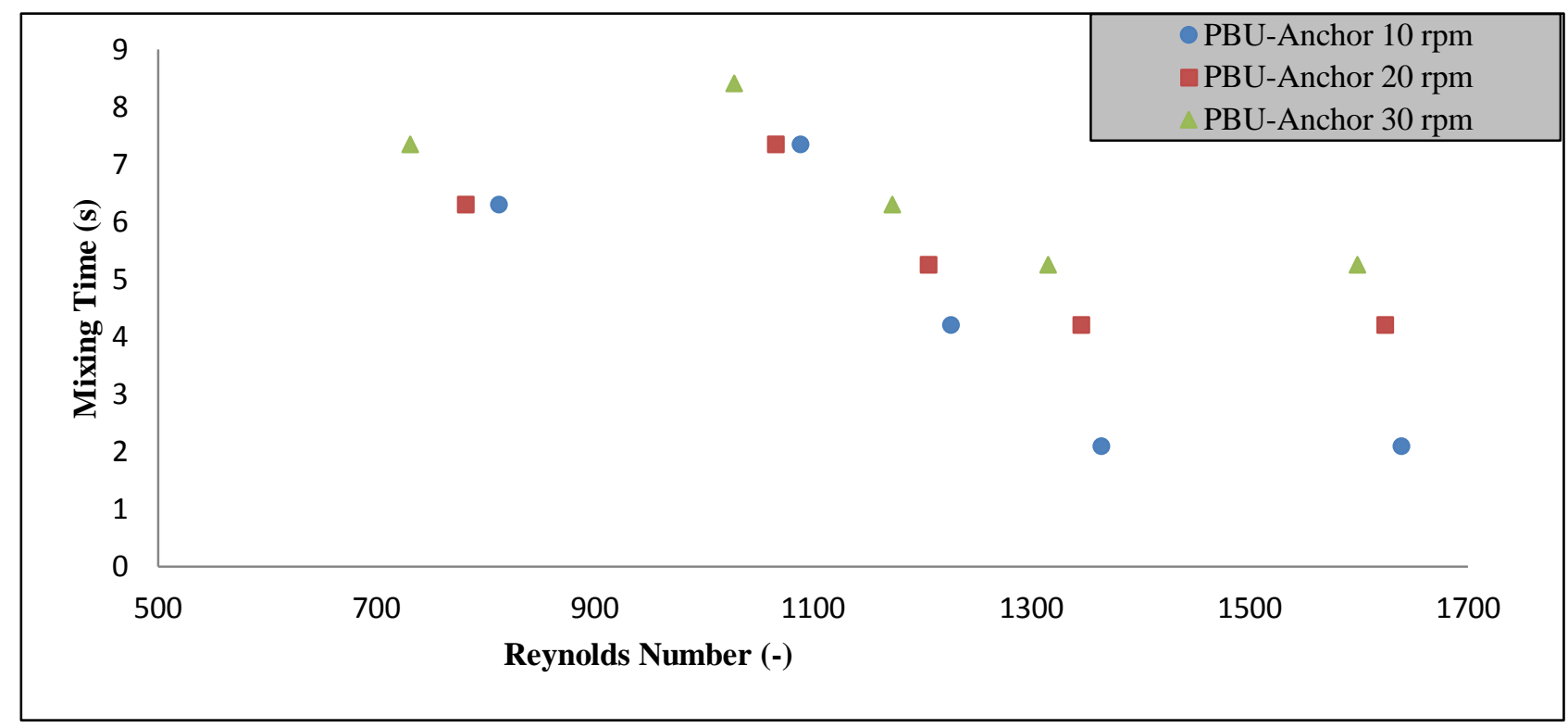

(a)

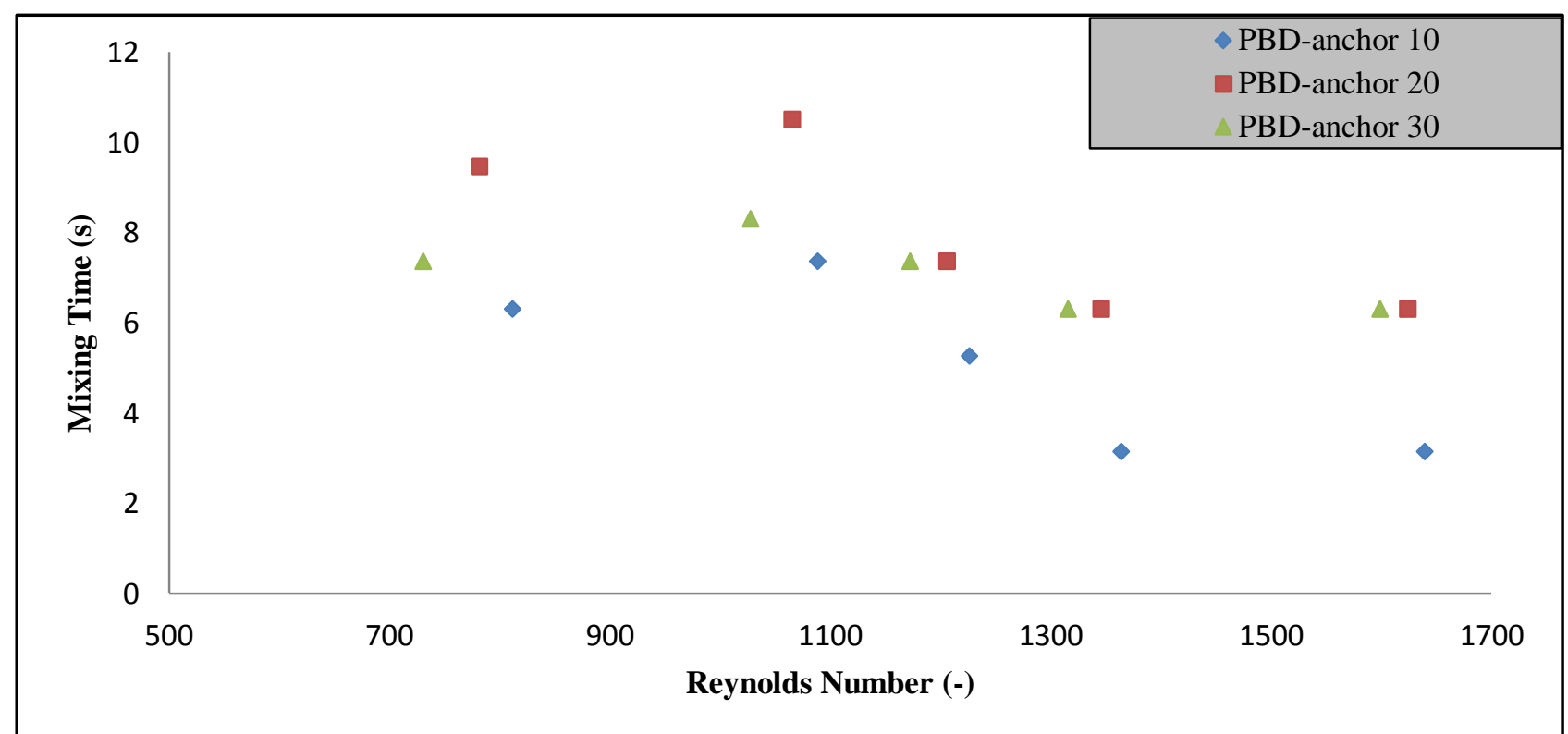

(b)

Figure (5.1- 3). Mixing time versus Reynolds number as a function of the anchor speed at constant gas flow rate of $0.0283 \mathrm{~m}^{3} / \mathrm{min}$ for the coaxial mixing system equipped with (a) PBU-anchor and (b) PBDanchor impellers ( $\max \mathrm{STDEV} \approx 2.23 \%$ ). 
Chapter 5: Results and Discussion

\subsubsection{Effect of anchor speed}

Increasing the Reynolds number in Figure 5.1-4 corresponds to the increasing of the anchor speed at the constant gas flow rate of $0.0283 \mathrm{~m}^{3} / \mathrm{min}$ and the central impeller speed of $135 \mathrm{rpm}$. These results show that increasing the anchor speed up to $20 \mathrm{rpm}(\operatorname{Re} \approx 1248)$ decreased the mixing time for both the PBU-anchor and the PBD-anchor coaxial mixers in the counter-rotating mode. However, mixing time was increased beyond the critical anchor speed of $20 \mathrm{rpm}$. Based on the literature, this can be attributed to the competition between the flows generated by each impeller throughout the vessel, which resulted in the reduction of the pumping capacity of the central impeller (Lee et al., 1957). Nevertheless, in the co-rotating mode, increasing the anchor speed (i.e. decreasing the Reynolds number) reduced the mixing time. The possible explanation can be that in the co-rotating mode stronger axial and tangential velocities are generated throughout the mixing vessel, leading to an increase in the size of the well-mixed regions. In addition, Rivera et al. (2009) demonstrated that in the counter-rotating mode the pressure gradients of the central impeller and anchor are in the opposite direction. Therefore, the discharge flow from the centered impeller encounters a higher pressure zone formed by the anchor resulting in a repulsive effect, which increases the mixing time. Lee et al. (1957) found that for an anchor agitated vessel, plots of mixing time versus Reynolds number reached to a minimum as agitator speed increased and then increased above a certain speed. Foucault et al. (2004) investigated the performance of the coaxial systems equipped with different central impellers including the Deflo, the Sevin, and a new hybrid impeller for both Newtonian and non-Newtonian fluids. In the case of Newtonian fluid, they came to a conclusion that the mixing times measured for the abovementioned coaxial mixers approached to a maximum by increasing the anchor speed up to $40 \mathrm{rpm}$ in counter-rotating mode. Further increase in the anchor speed resulted in a decrease in the mixing time. However, in co- 
rotating mode, the mixing time values measured for these three coaxial mixers showed a decreasing trend with an increase in the rotational speed of the anchor impeller. In the case of nonNewtonian fluids agitated in the counter-rotating mode, the mixing times for the aforementioned three coaxial mixers showed a decreasing trend up to the anchor speed of $20 \mathrm{rpm}$. At the anchor speed higher than this critical speed $(20 \mathrm{rpm})$, the mixing times started increasing with further increase in the anchor impeller speed.

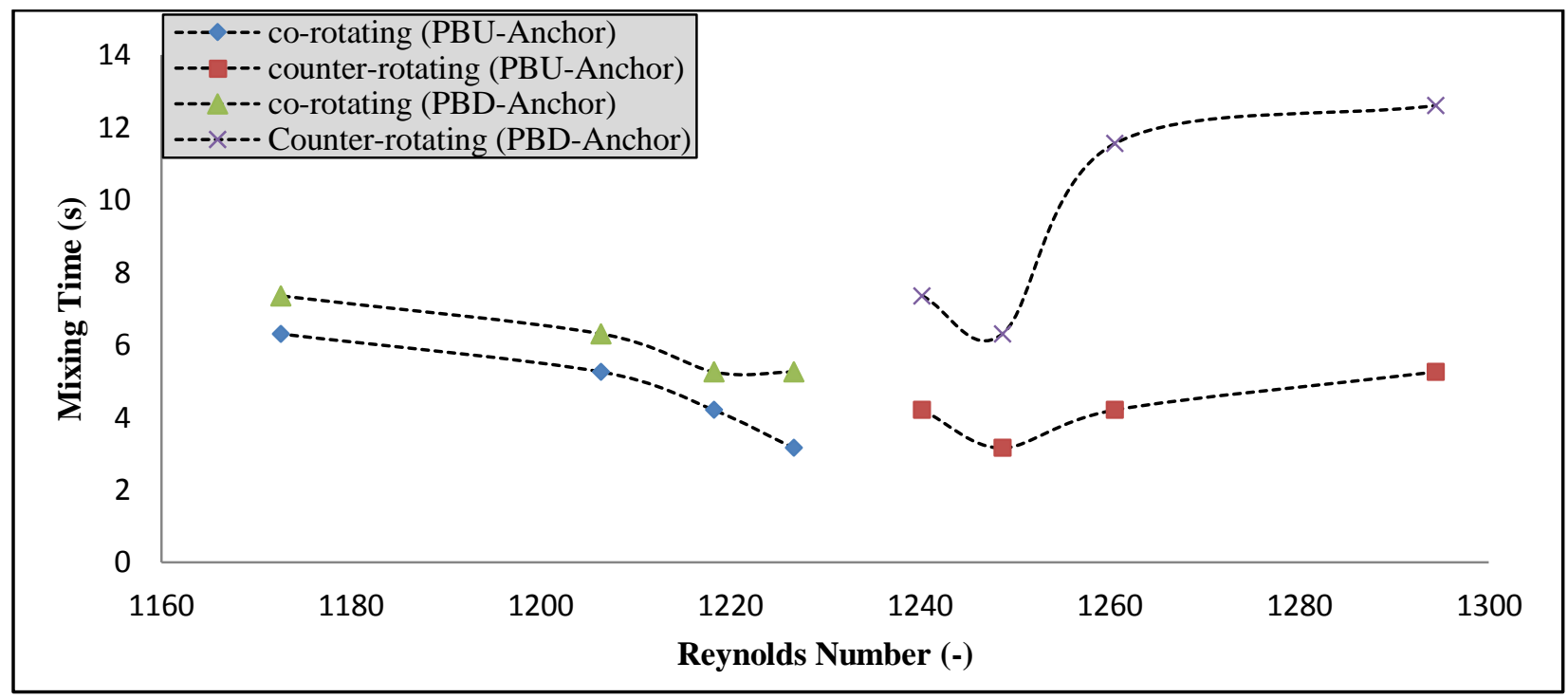

Figure (5.1- 4). Mixing time versus Reynolds number when only the anchor speed changes at the constant central impeller speed of $135 \mathrm{rpm}$ and the fixed gas flow rate of $0.0283 \mathrm{~m}^{3} / \mathrm{min}$ for the PBU-anchor and the PBD-anchor coaxial mixers for both co-rotating and counter-rotating modes (max $\mathrm{STDEV} \approx 4.76 \%$ ).

\subsubsection{Aerated mixing time}

Figure 5.1-5 exhibits how gassing affects the mixing times achieved by the PBD-anchor and PBU-anchor coaxial mixers at the central impeller speed of $135 \mathrm{rpm}$ and the anchor speed of 10 rpm. As it can be seen, increasing the gas flow number led to an increase in the mixing time for both coaxial configurations. By increasing the gas flow rate, the impeller pumping capacity was 


\section{Chapter 5: Results and Discussion}

drastically reduced, causing a rise in the mixing time. However, at the gas flow numbers greater than 2 , the mixing time was decreased by increasing the gas flow rate. In fact, at the higher gassing rates, the mixing hydrodynamics inside the vessel was imposed by the buoyancy force of the gas flow and thus the impeller did not disperse the gas properly and the gas-liquid system was flooded (Machon \& Jahoda, 2000). However, this condition is not desirable since it is related to the transition from dispersion to flooding. In addition, from the data presented in Figure 5.1-5, it can be seen that in the presence of gas, the PBU-anchor mixer showed better performance in comparison with the PBD-anchor mixer. The reason is that the PBD impeller generated the downward axial flow opposing the upward gas flow. Therefore, the competition between two opposite flows resulted in a lower pumping capacity and hence a higher mixing time. There is controversy regarding the effect of gassing on the blending time of mechanical agitated vessels. Some conducted works reported that the aeration enhanced the mixing times (Einsele and Finn, 1980; Blakebrough \& Sambamurthy, 1966). On the other hand, Abrardi et al. (1990) and Cronin et al. (1994) reported that the effect of gassing on the mixing time is complex and depends on the hydrodynamics occurring in the vessel, which was in agreement with the obtained results in the current work. Vasconcelos et al. (1995) reported that aeration can have positive, negative, and neutral effect on the mixing time depends on the flow patterns induced by the impellers, aeration or the combination of both. Micheau et al. (1995) showed that the aeration impact is a function of rheology of the fluids as shear-thinning behavior reduces the effect of aeration, and under these circumstances the influence of stirring on the blending time is dominant. 


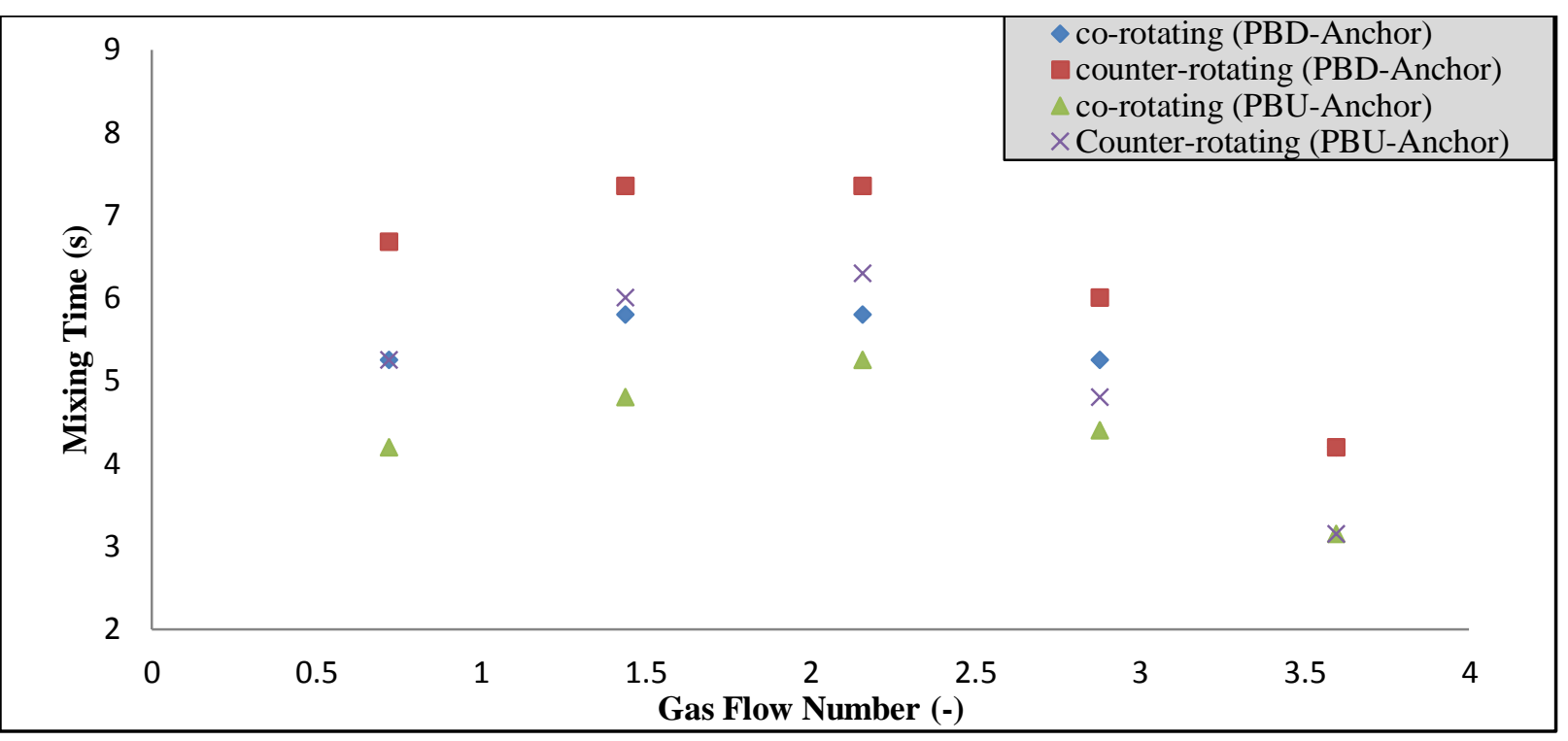

Figure (5.1- 5). Mixing time versus gas flow number at the anchor speed of $10 \mathrm{rpm}$ and the central impeller speed of $135 \mathrm{rpm}(\max \mathrm{STDEV} \approx 1.1 \%)$.

Figure 5.1-6 depicts the effect of the gas flow number on the mixing time of the aerated coaxial mixing system at different hydrodynamics regimes: flooding, loading and complete dispersion. It can be observed from Figures 5.1-6a and 5.1-6b that the mixing time reduced by an increase in the gas flow number for PBU-anchor and PBD-anchor impellers when the central and the anchor impellers were rotated at the low speeds of 90 and $10 \mathrm{rpm}$, respectively. At the lower central and anchor impeller speeds flooding regime was always observed. Flooding regime should be prevented because the impeller is surrounded by gas and therefore there is no proper contact between gas and liquid. Figures 5.1-6c and 5.1-6d show that at the high central impeller speed (180 rpm), loading/dispersion flow pattern was achieved at the lower gas flow numbers and both PBD-anchor and PBU-anchor impellers effectively dispersed the gas throughout the tank. In this hydrodynamic condition, increasing the gassing reduced the pumping capacity of the impeller. As a result, the transition between complete dispersion and loading happened which led to an increase in mixing time. Also, it should be mentioned that both coaxial mixing configurations showed the 
Chapter 5: Results and Discussion

same capability to handle the gassing before flooding happened at $\mathrm{F} 1 \approx 2.88$. As a conclusion, aeration does not always affect the blending time in the same way and no general behavior can be outlined. However, it can be concluded that the PBU-anchor coaxial mixer was more effective compared with the PBD-anchor coaxial mixer under the aeration conditions.

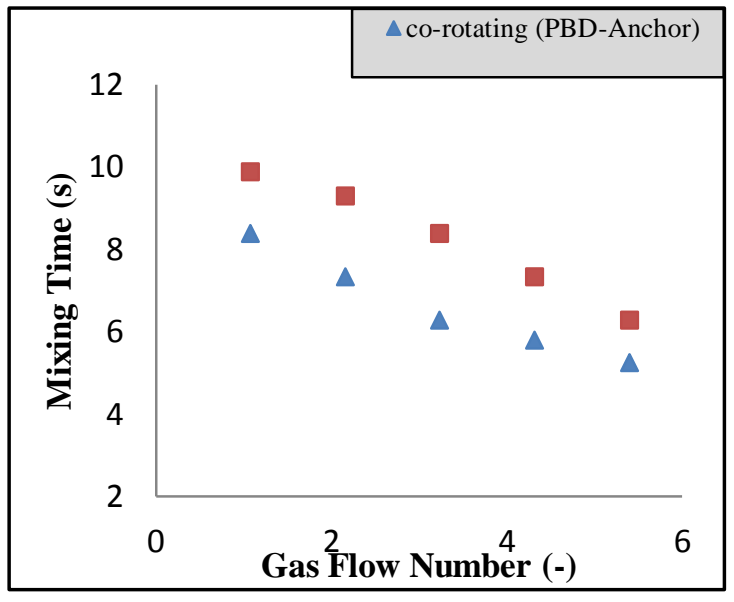

(a)

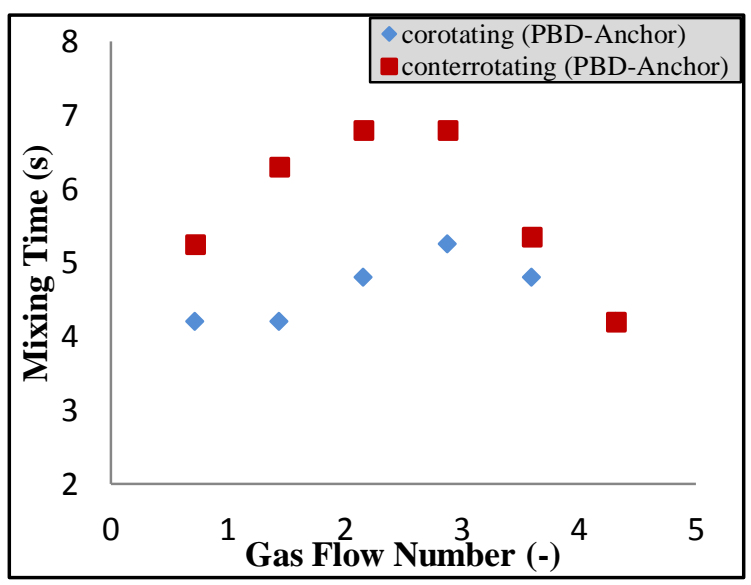

(c)

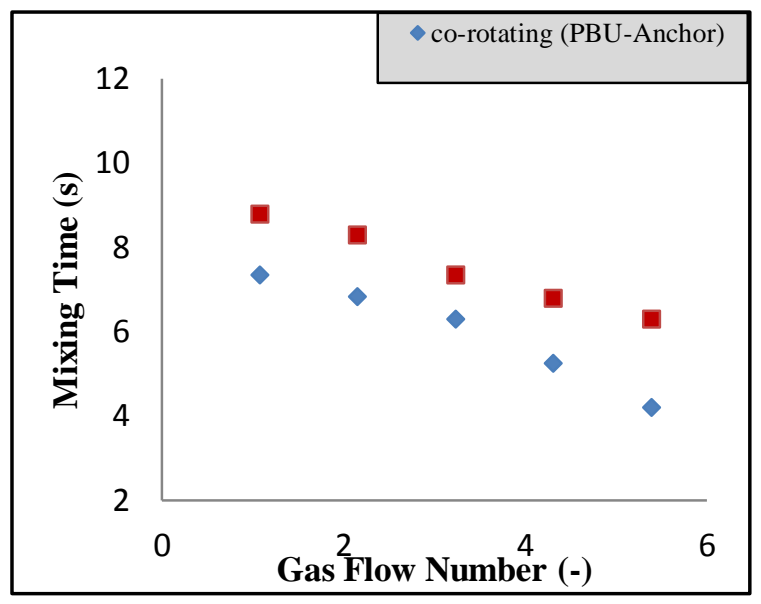

(b)

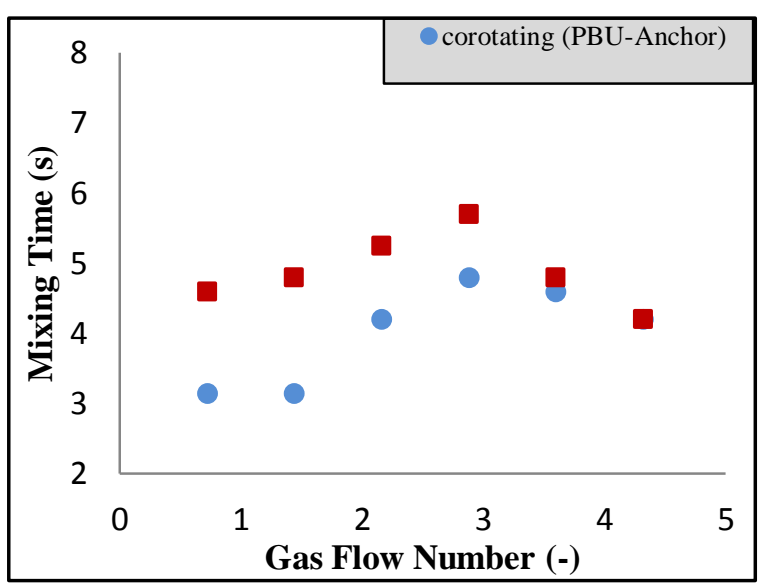

(d)

Figure (5.1- 6). Effect of gassing on the mixing time at the anchor speed of $10 \mathrm{rpm}$ and the central impeller speed of (a) PBD (90 rpm) (max STDEV $24.16 \%$ ), (b) PBU (90 rpm) (max STDEV 2.08\%), (c) PBD (180 rpm) (max STDEV $1.16 \%$ ), and (d) PBU (180 rpm) (max STDEV $24.13 \%)$. 
Chapter 5: Results and Discussion

\subsubsection{Power requirement}

In addition to the mixing time, the power uptake is another significant design parameter which should be considered in the selection of the most efficient aerated coaxial mixers. In this work, the correlation proposed by Bao et al. (2011) was used to obtain the power number of the anchor-PBD and anchor-PBU coaxial mixers agitating the corn syrup fluids with the viscosities in the range of 0.05-1.4 Pa.s (Eq. 5.1-2). It should be mentioned that three anchor speeds of 10, 20, and $30 \mathrm{rpm}$ and two different gas flow rates, 0.0189 and $0.0283 \mathrm{~m}^{3} / \mathrm{min}$, were considered to compare the power uptake of the aerated coaxial mixers. As mentioned before, the coaxial mixers are comprised of two independent shafts; top shaft is fitted with the central impeller rotating at a higher speed whereas the bottom shaft is equipped with the anchor stirring at a lower speed. Therefore, the total gassed power of the coaxial mixer is the sum of the power uptakes of the central impeller and the anchor. The purpose of Figure 5.1-7 was to show that the contribution of the central impeller power consumption to the total gassed power uptake of the coaxial mixer $\left(P_{c t}=P_{c} / P_{g t o t}\right)$ was more significant compared with that of the anchor impeller. For both coaxial mixers used in this study, the power uptake of the anchor (at a constant speed) progressively decreased at the higher central impeller speed; resulting in less contribution of the anchor power to the total gassed power. Therefore, at the higher central impeller speeds the ratio of the central impeller power to the total power is close to one, which represents the dominance of the central impeller in the coaxial mixing vessel at high speed ratios. Consequently, the total gassed power of the coaxial mixer is more affected by the central impeller than by the wall scraping anchor. 


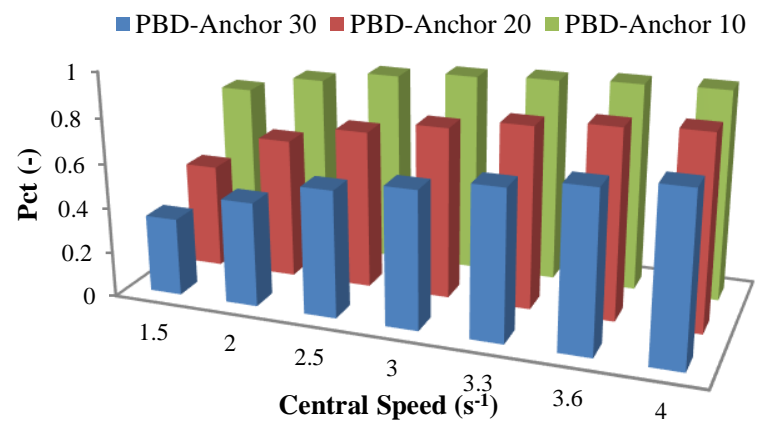

(a)

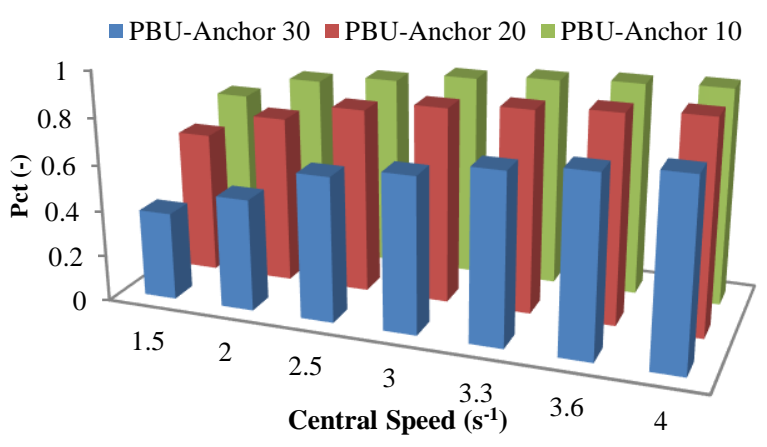

(b)

Figure (5.1- 7). Central impeller power contribution into the total gassed power of the aerated coaxial mixing vessel at constant gas flow rate $0.0283 \mathrm{~m}^{3} / \mathrm{min}$ and viscosity 0.7 Pa.s: (a) PBDAnchor, (b) PBU-Anchor.

Figure 5.1-8 was depicted in order to compare the power consumption of the PBU-anchor and the PBD-anchor operating in the counter-rotating and co-rotating modes. As expected, the power uptake of the aerated coaxial mixer was higher for more viscous fluids. It can also be observed that the total gassed power increased in the counter-rotating mode. This can be attributed to the inverse repulsive force, which was exerted by the central impeller on the anchor. As demonstrated in the previous sections, mixing time of the counter-rotating mode was also higher than that in the corotating mode. Therefore, co-rotating mode is the preferred rotating direction. Furthermore, the comparison between the power consumptions of the PBD-anchor and PBU-anchor revealed that the power drawn by the PBD-anchor was slightly higher than that of the PBU-anchor in both counter-rotating and co-rotating modes. Besides, the aerated coaxial mixing vessel equipped with the PBD-anchor was subject to severe torque and power instabilities especially at the higher gas flow rates. As a result, the coaxial mixer, which was furnished with the PBU as the central impeller, proved to be more efficient due to the lower power consumption and mixing time regardless of the fluid viscosity. 


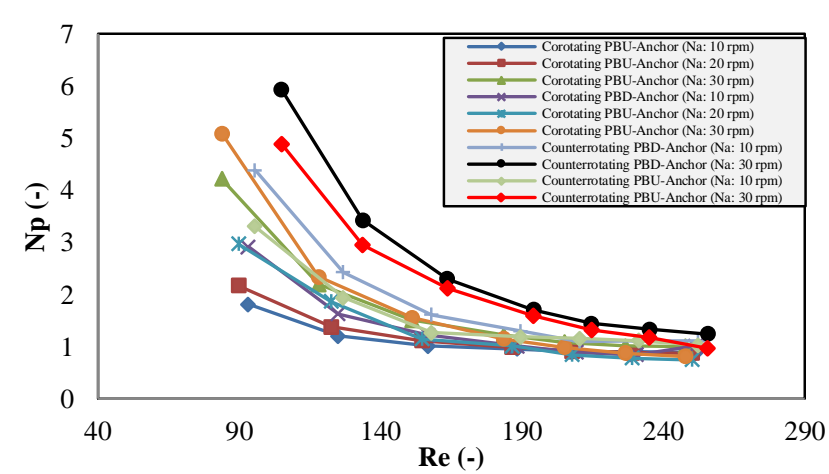

(a)

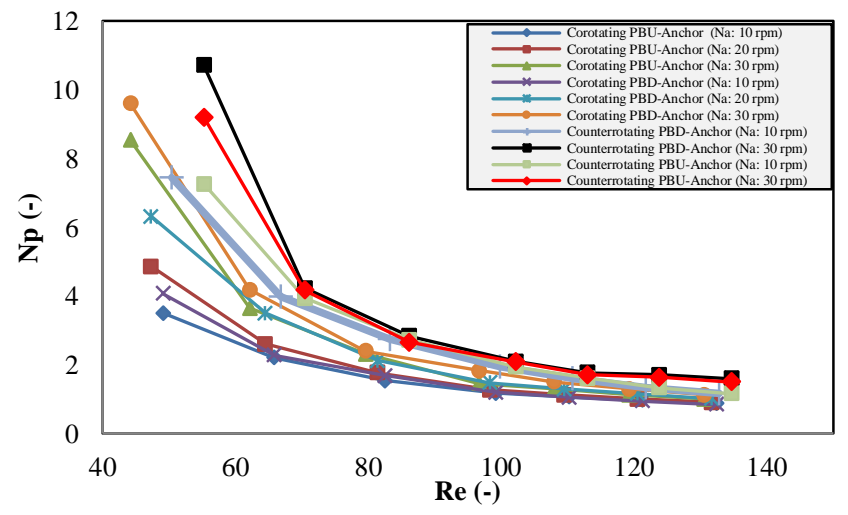

(c)

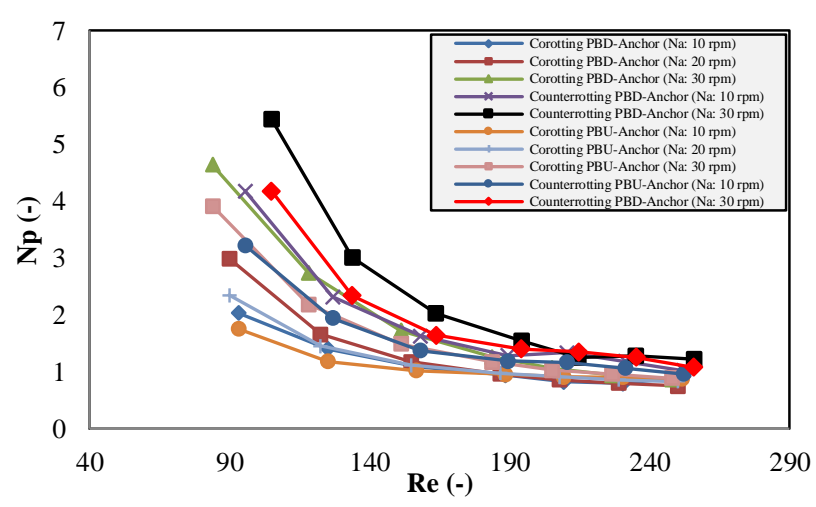

(b)

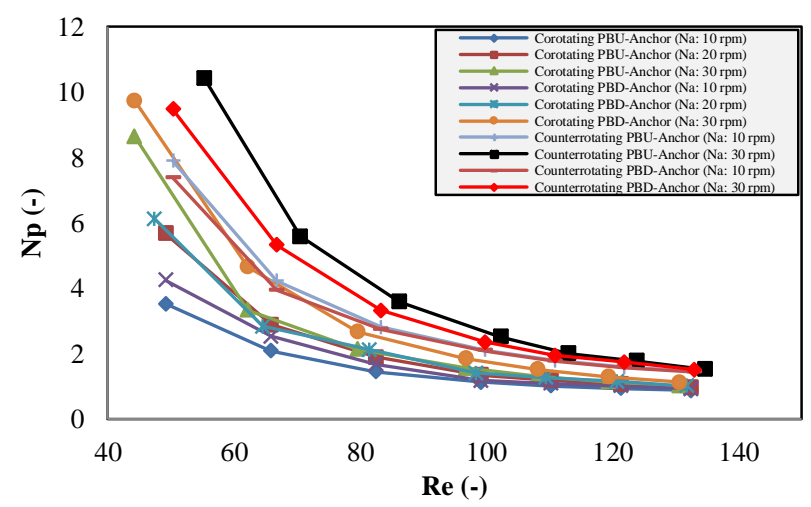

(d)

Figure (5.1- 8). Power number vs. Reynolds number for the aerated BPU-anchor and PBDanchor: (a) and (c) medium and high viscosities at gas flow rate $0.0189 \mathrm{~m}^{3} / \mathrm{min}$, (b) and (d) medium and high viscosity at gas flow rate $0.0283 \mathrm{~m}^{3} / \mathrm{min}$.

\subsubsection{Effect of viscosity}

Figure 5.1-9 exhibits a set of experiments carried out for the aerated coaxial mixer to evaluate the effect of viscosity on mixing time. In this case, the PBU or the PBD was employed along with the wall scraping anchor to stir the viscous corn syrup solutions. As expected, the mixing time increased with an increase in the fluid viscosity. The results contributed to the damping effect of the higher viscosity on the flow field and turbulence developed in the aerated coaxial mixer, leading to an increase in the mixing time. Besides, it was noticed that the viscosity affected the onset of dispersion in the aerated coaxial mixers. Figure 5.1-9 demonstrates that at the higher viscosity, 1.4 Pa.s, the transition from flooding to loading happened at the central impeller speed 
Chapter 5: Results and Discussion

equal to $180 \mathrm{rpm}$, the turning point of the curve $\left(\mathrm{N}_{\mathrm{D}}\right)$, which was higher than that captured in the case of lower viscosity, 0.05 Pa.s (see Section 5.1.2.2). Therefore, the transition between a less to a more dispersed regime was related to the type of the central impeller and the viscosity of the fluid being stirred. As demonstrated before, the effect of the anchor on the onset of dispersion was negligible; therefore only anchor speed of $10 \mathrm{rpm}$ was employed to evaluate the effect of viscosity. Also, it was shown that at the same power input, the mixing time obtained using the PBU-anchor was less than that of the PBD-anchor for both low and high viscosity corn syrup solutions. Therefore, in the aerated coaxial mixing vessel, the performance of the PBU-anchor was superior. In Figure 5.1-10, two different types of the coaxial mixing systems were compared in terms of their ability to handle the aeration before flooding. As it can be seen, flooding happened at flow number 2.16, irrespective of the pumping direction of the central impeller. From Figures 5.1-9 and 5.1-10, it was revealed that the fluid viscosity affected the capability of the impeller to fully disperse the gas phase in the liquid inside the mixing vessel. In other words, at the same operating conditions (e.g. central impeller speed, anchor speed, and gas flow rate), the transition from complete/loading gas flow pattern to flooding took place at aeration numbers equal to 2.16 and 2.88 for the high and low viscosity corn syrup solutions, respectively. Therefore, the pumping capacity of the pitched blade turbine was directly proportional to its speed, and inversely proportional to the gas flow rate and the viscosity of the stirred fluid. 
Chapter 5: Results and Discussion

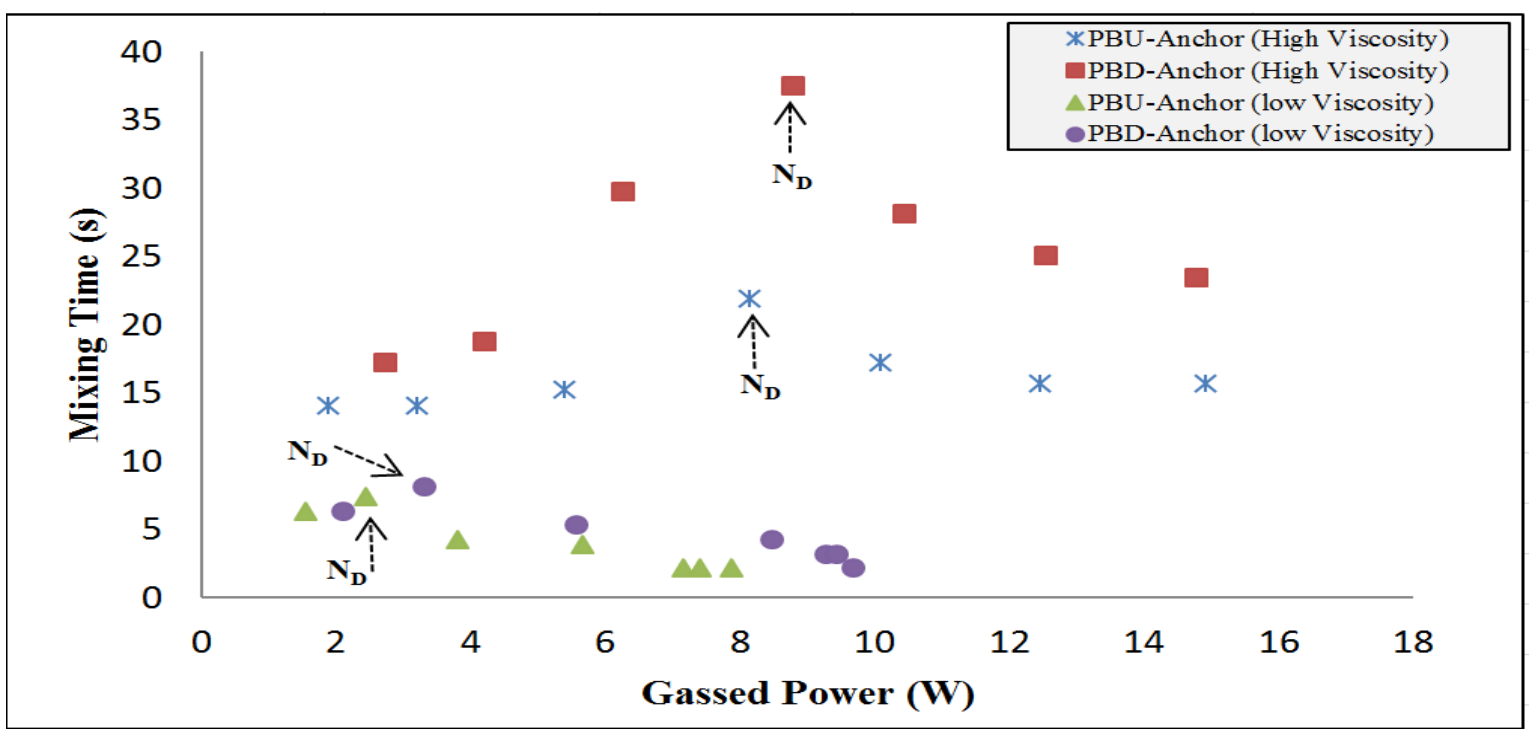

Figure (5.1- 9). Mixing time vs. power consumption of the aerated coaxial mixing vessel containing corn syrup solutions with the viscosities of 0.05 and 1.4 Pa.s (max STDEV $\approx 5 \%$ ).

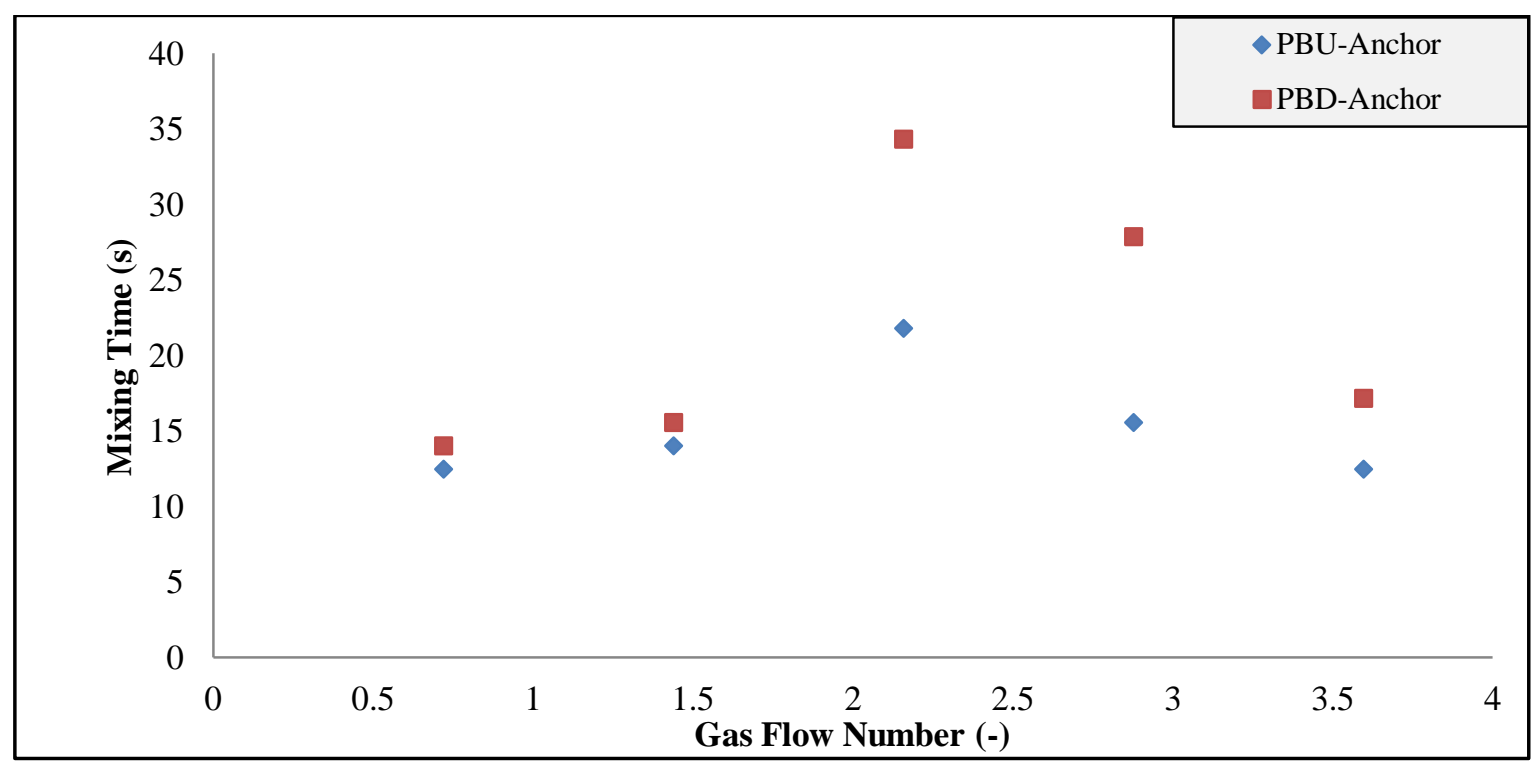

Figure (5.1- 10). Effect of gassing on the mixing time of the PBU-anchor and PBD-anchor coaxial mixers ( $N_{c}=180 \mathrm{rpm}$ and $N_{a}=10 \mathrm{rpm} \mu=1.4$ Pa.s) (max STDEV $\approx 2.17 \%$ ). 


\subsubsection{Response surface methodology model development}

In the previous section, it was shown that the PBU-anchor coaxial mixer in co-rotating mode is more effective than the PBD-anchor coaxial mixer in terms of mixing time and power uptake. Thus, only PBU-anchor mixer is studied in this section. Table 5.1-1 summarizes the actual and predicted results for the mixing time of the aerated co-axial mixer through Box-Behnken model. It can be observed that the deviations of predicted values from actual ones are reasonable. Therefore, the selected model could predict the response function reliably.

Table (5.1- 1). Four factor Box-Behnken design showing independent variables level and the observed and predicted mixing times.

\begin{tabular}{cccccccc}
\hline & \multicolumn{3}{c}{ Independent Variables } & \multicolumn{3}{c}{ Mixing Time } \\
\hline Run & $\begin{array}{c}\text { Central impeller } \\
\text { speed (rps) }\end{array}$ & $\begin{array}{c}\text { Anchor speed } \\
(\mathrm{rps})\end{array}$ & $\begin{array}{c}\text { Gas flow rate } \\
\left(\mathrm{m}^{3} / \mathrm{min}\right)\end{array}$ & $\begin{array}{c}\text { Viscosity } \\
(\text { Pa.s })\end{array}$ & $\begin{array}{c}\text { Experimental } \\
(\mathrm{s})\end{array}$ & $\begin{array}{c}\text { Predicted } \\
(\mathrm{s})\end{array}$ & $\begin{array}{c}\text { Absolute } \\
\text { Error } \\
(\%)\end{array}$ \\
& & & & & & & \\
$\mathbf{1}$ & 2.25 & 0.33 & 0.0094 & 0.05 & 5.25 & 4.33 & 17.52 \\
$\mathbf{2}$ & 2.25 & 0.33 & 0.0283 & 0.70 & 9.35 & 9.66 & 3.32 \\
$\mathbf{3}$ & 1.50 & 0.33 & 0.0094 & 0.70 & 12.60 & 13.63 & 8.17 \\
$\mathbf{4}$ & 1.50 & 0.33 & 0.0472 & 0.70 & 9.35 & 8.73 & 6.63 \\
$\mathbf{5}$ & 2.25 & 0.17 & 0.0472 & 0.70 & 7.35 & 7.37 & 0.27 \\
$\mathbf{6}$ & 3.00 & 0.33 & 0.0283 & 0.05 & 3.15 & 3.77 & 19.68 \\
$\mathbf{7}$ & 3.00 & 0.17 & 0.0094 & 1.4 & 12.48 & 13.57 & 8.73 \\
$\mathbf{8}$ & 2.25 & 0.50 & 0.0094 & 0.70 & 10.38 & 11.83 & 13.97 \\
$\mathbf{9}$ & 1.50 & 0.17 & 0.0283 & 0.70 & 11.55 & 10.22 & 11.52 \\
$\mathbf{1 0}$ & 2.25 & 0.33 & 0.0283 & 0.70 & 8.40 & 9.66 & 15.00 \\
$\mathbf{1 1}$ & 3.00 & 0.33 & 0.0283 & 1.40 & 19.74 & 19.38 & 1.82 \\
$\mathbf{1 2}$ & 2.25 & 0.17 & 0.0283 & 0.05 & 3.15 & 3.37 & 6.98 \\
$\mathbf{1 3}$ & 2.25 & 0.33 & 0.0283 & 0.70 & 8.40 & 9.66 & 15.00 \\
$\mathbf{1 4}$ & 3.00 & 0.33 & 0.0094 & 0.70 & 7.35 & 7.85 & 6.80 \\
$\mathbf{1 5}$ & 2.25 & 0.17 & 0.0094 & 0.70 & 9.35 & 7.53 & 19.47 \\
$\mathbf{1 6}$ & 2.25 & 0.33 & 0.0283 & 0.70 & 8.40 & 9.66 & 15.00 \\
$\mathbf{1 7}$ & 2.25 & 0.33 & 0.0283 & 1.40 & 17.85 & 18.01 & 0.90 \\
$\mathbf{1 8}$ & 3.00 & 0.33 & 0.0472 & 0.70 & 9.35 & 9.27 & 0.86 \\
$\mathbf{1 9}$ & 2.25 & 0.50 & 0.0472 & 0.70 & 7.35 & 8.51 & 15.78 \\
$\mathbf{2 0}$ & 1.50 & 0.33 & 0.0283 & 1.40 & 17.85 & 19.77 & 10.76 \\
$\mathbf{2 1}$ & 2.25 & 0.33 & 0.0283 & 0.70 & 8.40 & 9.66 & 15.00 \\
$\mathbf{2 2}$ & 1.50 & 0.33 & 0.0283 & 1.40 & 19.95 & 19.77 & 0.90 \\
$\mathbf{2 3}$ & 2.25 & 0.33 & 0.0472 & 0.05 & 4.20 & 4.11 & 2.14 \\
$\mathbf{2 4}$ & 2.25 & 0.50 & 0.0283 & 0.05 & 5.25 & 5.01 & 4.57 \\
$\mathbf{2 5}$ & 2.25 & 0.50 & 0.0283 & 1.40 & 18.90 & 19.47 & 3.02 \\
$\mathbf{2 6}$ & 3.00 & 0.17 & 0.0283 & 0.70 & 7.35 & 6.74 & 8.30 \\
$\mathbf{2 7}$ & 3.00 & 0.50 & 0.0283 & 0.70 & 9.35 & 10.32 & 10.37 \\
$\mathbf{2 8}$ & 1.50 & 0.50 & 0.0283 & 0.70 & 11.55 & 12.08 & 4.59 \\
$\mathbf{2 9}$ & 2.25 & 0.33 & 0.0472 & 1.4 & 14.04 & 15.97 & 13.75 \\
\hline & & & & & & \\
\end{tabular}


Chapter 5: Results and Discussion

\subsection{Statistical analysis}

As mentioned before, a second-order polynomial model with quadratic and interaction terms, Eq. (5.1-5), can be used to fit the actual and coded values of experimental data. Regarding the significance of the applied model, the analysis of variance (ANOVA) was used. The estimated regression coefficients along with different descriptive statistics such as $\mathrm{p}$ value, the degree of freedom $(d f)$, coefficient of determination $\left(\mathrm{R}^{2}\right)$, the adjusted coefficient of determination (Adj. $\mathrm{R}^{2}$ ), the coefficient of variance $(C V)$, and mean square are given in Table 5.1-2. As it can be seen, the probability value is less than 0.0001 , which demonstrates that the model is remarkably accurate and could be utilized to predict the mixing time. Also, the adequacy of the fitting model to actual data can be analysed by the coefficient of determination $\left(\mathrm{R}^{2}\right)$. Since both $\mathrm{R}^{2}(0.9680)$ and adjusted $\mathrm{R}^{2}(0.9361)$ approached to unity, the compatibility of the model to the experimental data was justifiable. Therefore, mixing time could be predicted quite well by the suggested model. The higher values of the adequate precision which measures the signal to noise ratio (in the current work 19.251) show that the model is desirable. The other factor that should be considered to verify the adequacy of the model is the lack-of-fit. The lack-of-fit of the model was 1.95 which meant this term was not significant compared to the pure error. Therefore, the developed quadratic model can be expressed as:

$$
\begin{aligned}
& Y=9.66-1.31 X_{1}+1.36 X_{2}-0.87 X_{3}+6.69 X_{4}+0.43 X_{1} X_{2}+1.58 X_{1} X_{3}+0.17 X_{1} X_{4}-0.79 X_{2} X_{3} \\
& +0.54 X_{2} X_{4}-0.76 X_{3} X_{4}+0.62 X_{1}^{2}-0.44 X_{2}^{2}-0.41 X_{3}^{2}+1.66 X_{4}^{2}
\end{aligned}
$$

where $X_{1}, X_{2}, X_{3}$, and $X_{4}$ are coded terms of four independent variables indicating central impeller speed, anchor speed, gas flow rate, and viscosity, respectively. $Y$ is the reference function (mixing time). 
Table (5.1- 2). Analysis of variance for response surface quadratic model

\begin{tabular}{ccccc}
\hline Factor & df & Mean Square & F value & P value \\
\hline Model & 14 & 42.14 & 30.28 & $<0.0001$ significant \\
$\mathbf{X}_{\mathbf{1}}$-Central Speed & 1 & 20.88 & 15.00 & 0.0017 \\
$\mathbf{X}_{\mathbf{2}}$ - Anchor speed & 1 & 20.27 & 14.57 & 0.0019 \\
$\mathbf{X}_{\mathbf{3}}$ - Gas flow rate & 1 & 8.26 & 5.94 & 0.0288 \\
$\mathbf{X}_{\mathbf{4}}$ - Viscosity & 1 & 498.40 & 358.12 & $<0.0001$ \\
$\mathbf{X}_{\mathbf{1}} \mathbf{X}_{\mathbf{2}}$ & 1 & 0.78 & 0.56 & 0.4663 \\
$\mathbf{X}_{\mathbf{1}} \mathbf{X}_{\mathbf{3}}$ & 1 & 10.57 & 7.60 & 0.0154 \\
$\mathbf{X}_{\mathbf{1}} \mathbf{X}_{\mathbf{4}}$ & 1 & 0.12 & 0.085 & 0.7748 \\
$\mathbf{X}_{\mathbf{2}} \mathbf{X}_{\mathbf{3}}$ & 1 & 2.67 & 1.92 & 0.1880 \\
$\mathbf{X}_{\mathbf{2}} \mathbf{X}_{\mathbf{4}}$ & 1 & 0.93 & 0.67 & 0.4270 \\
$\mathbf{X}_{\mathbf{3}} \mathbf{X}_{\mathbf{4}}$ & 1 & 1.83 & 1.31 & 0.2711 \\
$\mathbf{X}_{\mathbf{1}}{ }^{2}$ & 1 & 2.44 & 1.75 & 0.2066 \\
$\mathbf{X}_{\mathbf{2}}{ }^{2}$ & 1 & 1.20 & 0.86 & 0.3693 \\
$\mathbf{X}_{\mathbf{3}}{ }^{2}$ & 1 & 1.07 & 0.77 & 0.3949 \\
$\mathbf{X}_{\mathbf{4}}{ }^{2}$ & 1 & 17.47 & 12.55 & 0.0032 \\
Residual $_{\text {Lack of fit }}$ & 14 & 1.39 & & \\
Pure error & 10 & 1.95 & & \\
$\mathbf{R}^{\mathbf{2}}$ & 4 & 0.000 & & \\
Adjusted $\mathbf{R}^{\mathbf{2}}$ & 0.9680 & & & \\
Adequate Precision & 0.9361 & & & \\
\hline
\end{tabular}

\subsection{Analysis of the model coefficient and their interactions}

Whenever the significance of the suggested model is ensured (Table 5.1-2) and good fit is achieved between actual and predicted data (Table 5.1-1), it is time to evaluate the effect of each parameter and their interactions on the response variable. The most dominant parameters on the aerated mixing time could be specified based on their F-values and P-values listed in Table 5.1-2. The greater the F-value and the lower the P-value, the more significant is the coefficient. As illustrated in Table 5.1-2, the model parameters with the P-values less than 0.05 are significant. Therefore, all considered independent factors had significant linear effects on the mixing time. The greatest F-value and the lowest P-value of the viscosity term suggested that the mixing time of the aerated coaxial mixing vessel was strongly affected by this factor. Although the gas flow rate had linear influence on the response function, its effect was less than the effects of the central impeller 
Chapter 5: Results and Discussion

speed, anchor speed, and viscosity. Positive sign in front of the terms shows a synergistic effect, while negative sign shows an opposite effect. Therefore, an increase in the anchor speed results in an increase in the mixing time. As mentioned before, when the speed ratio was decreased by increasing the anchor rotational speed the tangential flow created by the anchor impeller governed the flow pattern inside the tank, which led to an increase in the mixing time.

The interaction model parameters including $X_{1} X_{2}, X_{1} X_{4}, X_{2} X_{3}, X_{2} X_{4}$, and $X_{3} X_{4}$ did not show any significant effect on the response function and can be neglected. While, the interaction effect of the central impeller speed and the gas flow rate $\left(X_{1} X_{3}\right)$ exhibited a significant effect on the mixing time. In addition, the only quadratic parameter which affected the response function was $X_{4}^{2}$ and the other quadratic interactions were negligible.

\subsection{Three-dimensional response surfaces}

In order to facilitate the visualization of the effects of the independent variables on the mixing time function, the three dimensional response surfaces are depicted in Figure 5.1-11. These plots were created by changing two factors within the experimental ranges while the other was maintained at the central point of its range.

In the present work, both central and anchor speeds displayed considerable linear effect on the mixing time (Figure 5.1-11a). It should be mentioned that because of the negligible quadratic effects of aforementioned factors, there is no maximum and minimum in the mixing time at a certain point of the considered independent variables. As illustrated, the mixing time reduced at the higher central impeller speed levels. Nonetheless, the higher anchor speeds had a negative effect on the blending time. The signs of the obtained coefficients in Table 5.1-2 also confirm the synergetic and antagonistic effects of the central impeller and anchor speeds, respectively. 


\section{Chapter 5: Results and Discussion}

Figure 5.1-11b depicts the response surface of the effects of the gas flow rate and the central impeller speed on the mixing time. It was observed that at the lower impeller speeds, the gas flow governed the flow pattern inside the vessel and therefore the higher gas flow rates resulted in the lower mixing times. However, at the higher central impeller speeds, the flow pattern induced by the impeller was dominant. Under these circumstances, the mixing time decreased with an increase in central impeller speed.

As represented in Figure 5.1-11c, at the lower anchor speed (less than $0.15 \mathrm{~s}^{-1}$ ), the agitate vessel acted like a bubble column and increasing the gas flow rate caused a decrease in the mixing time. However, further increase in the anchor speed seemed to have the opposite effect on the mixing time because of the tangential flow pattern of the anchor, which governed the overall flow pattern inside the tank.

As illustrated in Figures 5.1-11d-f, the mixing time was significantly influenced by the viscosity. The mixing time was increased with an increase in the fluid viscosity. Also, it was observed that the interactions of the central impeller speed and anchor speed with the viscosity were more pronounced. Generally, for a high viscosity fluid, the improvement of the mixing at the higher central impeller speeds was related to the reduction of the stagnant zones due to the enhanced turbulent exchange. As mentioned before, the proportionality between the response function and the anchor speed was not strong enough to increase the bulk flow since the tangential flow of the anchor was dominant. 


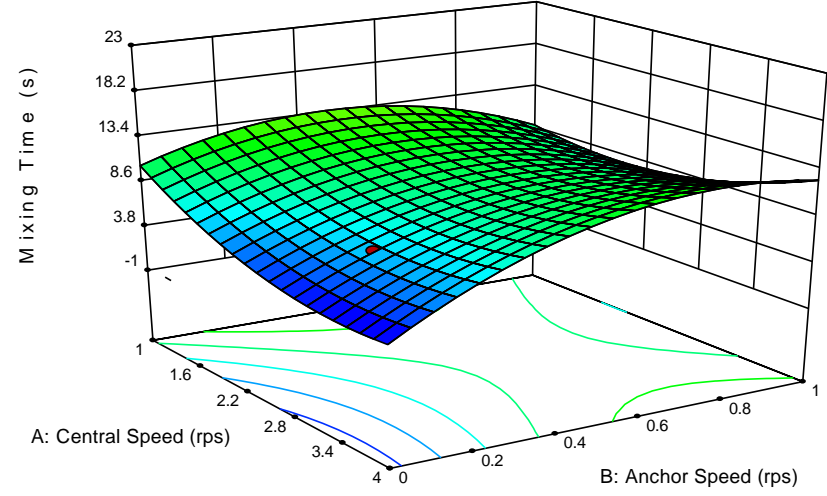

(a)

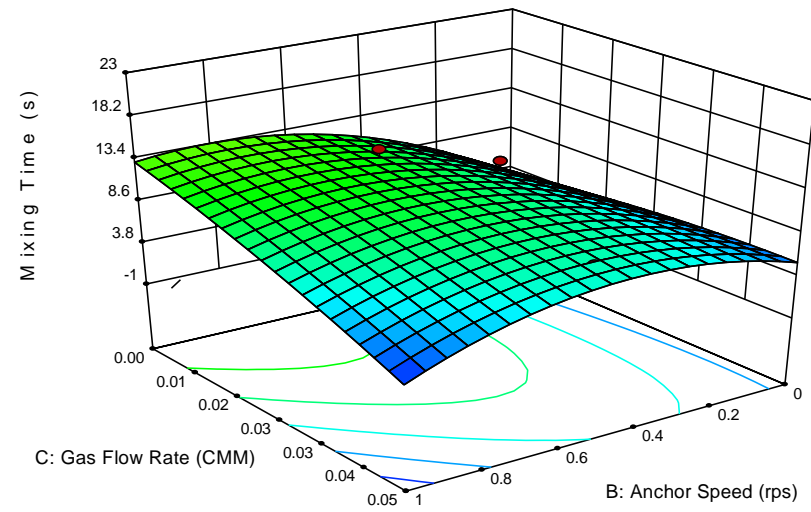

(c)

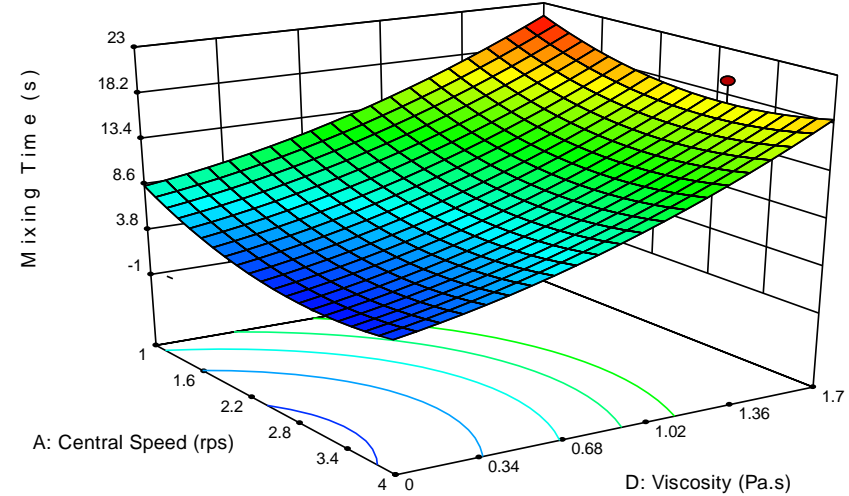

(e)

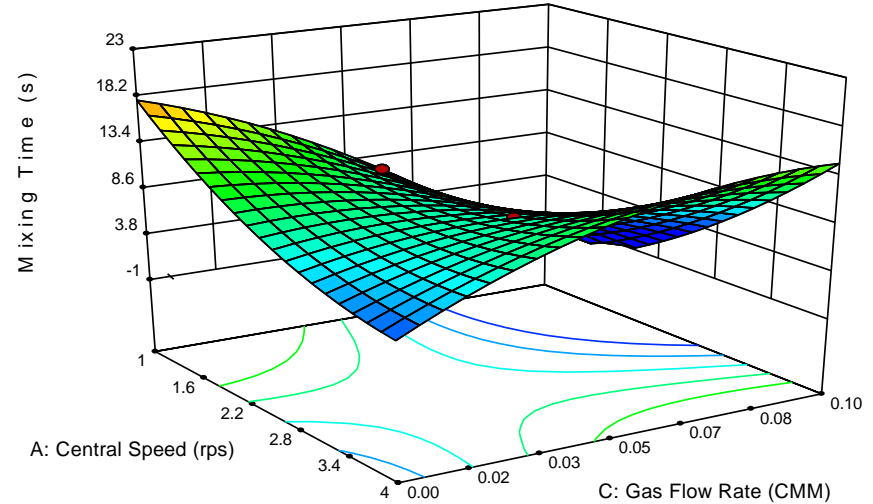

(b)

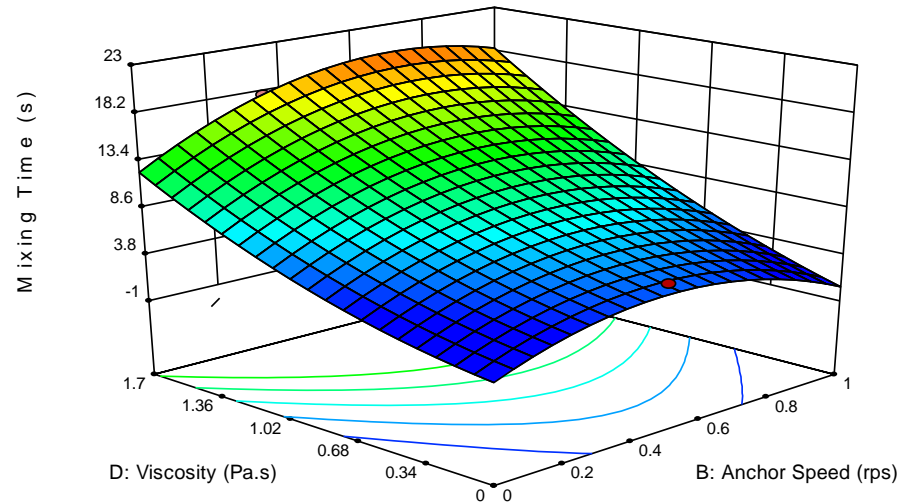

(d)

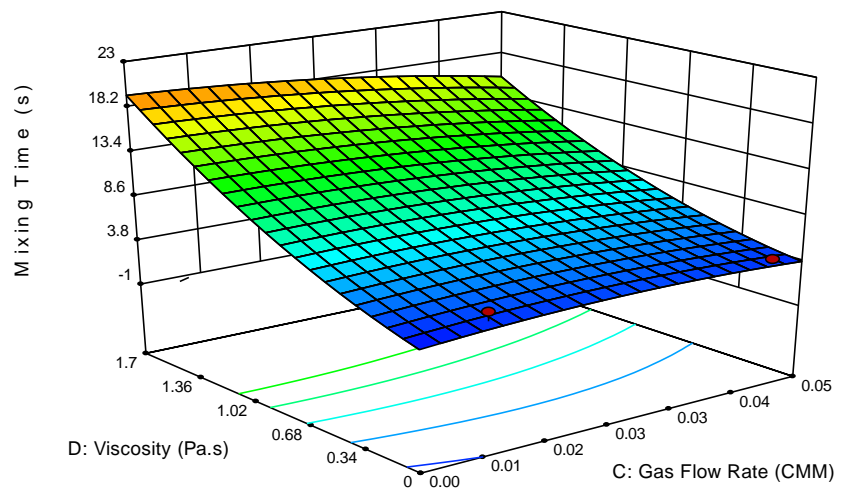

(f)

Figure (5.1- 11). Reference surfaces showing mixing time as a function of two independent variables: (a) Central impeller speed $\left(X_{1}\right)$ and anchor speed $\left(X_{2}\right)$, (b) central impeller speed $\left(X_{l}\right)$ and gas flow rate $\left(X_{3}\right),(\mathbf{c})$ anchor speed $\left(X_{2}\right)$ and gas flow rate $\left(X_{3}\right),(\mathbf{d})$ anchor speed $\left(X_{2}\right)$ and viscosity $\left(X_{4}\right)$, (e) central impeller speed $\left(X_{1}\right)$ and viscosity $\left(X_{4}\right)$, and (f) gas flow rate $\left(X_{3}\right)$ and viscosity $\left(X_{4}\right)$. 
Chapter 5: Results and Discussion

\subsubsection{Conclusions}

The objective of this study was to evaluate the liquid-gas mixing in a reactor equipped with a coaxial mixer through tomography technique. The performances of two types of the coaxial mixers, called anchor-PBU and anchor-PBD, were assessed in term of the mixing time and power consumption. The mixing times obtained by the coaxial mixers were shorter than those acquired by a single impeller system. Also, the PBU-anchor mixer outweighed the PBD-anchor mixer in the aerated vessel. In the presence of gas, the flow pattern induced by the impeller, gas or combination of both played a significant role in affecting the mixing time. In flooding regime, gassing showed positive effect on the blending time while in loading and complete dispersion conditions, the pumping capacity of the central impeller affected by gassing and led to a rise in mixing time. The onset of dispersion can be affected by the central impeller type, central impeller speed, and the viscosity of the fluid being agitated. It was also found that the fluid viscosity significantly affected the capability of the impeller to fully disperse the gas in the mixing vessel. At the higher fluid viscosities, the impeller can be flooded at the lower gas flow rates. Response surface methodology (RSM) was used to study the individual and interactive effects of the central impeller speed, the gas flow rate, the anchor speed, and viscosity on the mixing time. The developed quadratic model indicated a high coefficient of determination. It was found that the viscosity was the most significant factor while the gas flow rate was the least effective one. Considering the interaction effects, the central impeller speed and the gas flow rate showed the highest effect on the mixing time. 
Chapter 5: Results and Discussion

\subsection{Analysis of power consumption and gas holdup distribution for an aerated reactor equipped with a coaxial mixer: Novel correlations for the gas flow number and gassed power}

\subsubsection{Introduction}

With regards to the complexities arise from the presence of the gas and two independently rotating impellers; it is a difficult task to characterize the aerated coaxial mixing vessels in terms of power uptake. Several correlations have been reported for the dimensionless Reynolds and power numbers of the ungassed coaxial mixing vessel (Liu et al., 2013; Bao et al., 2011; Farhat et al., 2007; Foucault et al., 2006). All the published works demonstrated that the total power consumption was related to the interaction between the centered impeller and anchor, which can be varied by the system geometry and the speed ratio (central impeller speed over the anchor speed). It must be mentioned that it is not possible to apply the aforementioned correlations to the aerated coaxial mixing vessel due to the presence of the gas and its influence on the power dissipated by each impeller. Regarding the gassed power drawn of the agitated tanks, two types of correlations have been found in the literature, namely dimensional and dimensionless correlations (Linek et al., 1996; Cui et al., 1996; Abrardi et al., 1990; Michel and Miller, 1962). These correlations have been proposed for various single or multiple agitators placed on the same shaft and therefore they are not applicable to the aerated coaxial mixing vessels. As expressed before, the main problem is related to the presence of two independent shafts, the top shaft and the bottom shaft, for driving the centered impeller and the anchor at different speeds and directions. Therefore, the rotational speeds of the central impeller and the anchor should be converted into one equivalent rotational speed in order to predict the power uptake of the coaxial mixing vessel. In addition, the speed ratio between two impellers should take into account in both dimensional and dimensionless

analysis of the total gassed power drawn of the aerated coaxial mixing vessel. Interestingly, based 
Chapter 5: Results and Discussion

on the experimental observations, sparged gas affects the power consumption of the central impeller and the anchor in different manner. The abovementioned discussion directed us to develop the novel dimensional gassed power, and dimensionless gas flow number and power number correlations to find out how different parameters such as the central impeller speed, anchor speed, speed ratio, fluid viscosity, and gas flow rate can alter the power uptake of the aerated coaxial mixing vessel.

In addition to the power uptake, the gas holdup is defined as one of the most significant hydrodynamic parameters required for the reliable design of the aerated mixing vessels. Hence, a detailed knowledge of the local gas holdup and its variation with different operating conditions are necessary. In the current work, ERT was used for acquiring radial and axial distributions of gas holdup in the aerated coaxial mixing vessel.

Therefore, in order to overcome the problems associated with the prediction of the power uptake of the aerated coaxial mixer, we developed a new generalized gas flow number and power number to study the real behavior of the gas phase in the aerated coaxial mixing reactor. In addition, an empirical correlation for the estimation of the gassed power uptake of the aerated coaxial agitated tank as a function of the central impeller speed, anchor speed, speed ratio, fluid viscosity, and gas flow rate was proposed. Furthermore, in this research for the first time ERT was employed to characterize the effects of the central impeller speed, anchor speed, and speed ratio on the radial distribution of the sparged gas within the aerated coaxial mixing system. 
Chapter 5: Results and Discussion

\subsubsection{Results and Discussions}

\subsubsection{Power drawn}

In the literature, several works have been published on the power uptake of the single-shaft aerated mixing vessels, and there are a number of correlations for predicting their power drawn (Linek et al., 1996; Cui et al., 1996; Abrardi et al., 1990; Michel and Miller, 1962). To the best of our knowledge, no data has been presented concerning the power consumption of the aerated coaxial mixers. However, a few studies have focused only on the power consumption of the ungassed coaxial mixing vessels working with highly viscous Newtonian and non-Newtonian fluids (Liu et al., 2013; Bao et al., 2011; Farhat et al., 2007; Foucault et al., 2006).

In contrast to the gassed power of the traditional mixer, the prediction of the power drawn of the aerated coaxial mixer is a challenging task since the coaxial mixing vessel is facilitated with two independent impellers, which can rotate at different speeds and directions. Therefore, two rotational speeds of the centered impeller and the anchor should be converted into one equivalent rotational speed in order to predict the power uptake of the coaxial mixing vessel. Some researchers have tried to propose different correlations for the equivalent rotational speed, which are summarized in Table 5.2-1. In this table, the plus and minus signs are adopted for counter and corotating modes, respectively. $D_{c}$ is the central impeller diameter and $D_{a}$ is the anchor diameter. $N_{R}$ and $D_{N}$ are denoted as the speed and diameter ratios, respectively. 
Chapter 5: Results and Discussion

Table (5.2- 1). Equivalent rotational speed, $\mathrm{Re}$, and $\mathrm{N}_{\mathrm{p}}$ proposed for coaxial mixers.

\begin{tabular}{|c|c|c|c|}
\hline References & $\begin{array}{c}\text { Equivalent } \\
\text { rotational speed } \\
\end{array}$ & Reynolds number (-) & Power number (-) \\
\hline $\begin{array}{l}\text { Foucault et } \\
\text { al. (2006) }\end{array}$ & $\mathrm{N}_{\mathrm{c}} \pm \mathrm{N}_{\mathrm{a}}$ & $\operatorname{Re}=\frac{\rho\left(N_{c} \pm N_{a}\right) D_{c}^{2}}{\mu}$ & $N_{p}=\frac{P_{u g}}{\rho\left(N_{c} \pm N_{a}\right)^{3} D_{c}^{5}}$ \\
\hline $\begin{array}{l}\text { Farhat et al. } \\
(2007)\end{array}$ & $\frac{N_{c} D_{c}+N_{a} D_{a}}{D_{c}}$ & $\operatorname{Re}=\frac{\rho\left(N_{c} D_{c}+N_{a} D_{a}\right) D_{c}}{\mu}$ & $N_{p}=\frac{P_{u g}}{\rho\left(N_{c} D_{c}+N_{a} D_{a}\right)^{3} D_{c}^{2}}$ \\
\hline $\begin{array}{l}\text { Bao et al. } \\
(2011)\end{array}$ & $N_{c} \pm \frac{N_{a}}{N_{R}}$ & $\operatorname{Re}=\frac{\rho\left(N_{c} \pm N_{a} / N_{R}\right) D^{2}}{\mu}$ & $N_{p}=\frac{P_{u g}}{\rho\left(N_{c} \pm N_{a} / N_{R}\right)^{3} D_{c}^{5}}$ \\
\hline $\begin{array}{l}\text { Liu et al. } \\
\text { (2013) }\end{array}$ & $N_{c}+\left(\frac{1}{D_{N}} \pm \frac{1}{N_{R}}\right) N_{a}$ & $\operatorname{Re}=\frac{\rho\left(N_{c}+\left(1 / D_{N} \pm 1 / N_{R}\right) N_{a}\right)\left[1+\left(D_{N}-1 / N_{R}\right)\right]_{c}^{2} D_{c}^{2}}{\mu}$ & $N_{p}=\frac{P_{u g}}{\rho\left(\left(N_{c}+\left(1 / D_{N} \pm 1 / N_{R}\right) N_{a}\right)^{3}\left[1+\left(D_{N}-1 / N_{R}\right)\right]^{5}{ }_{c}^{5}\right.}$ \\
\hline
\end{tabular}

In order to evaluate the applicability of the proposed equivalent rotational speeds in measuring the power consumption of the aerated coaxial mixing vessel, the power curve $\left(\operatorname{Re}\right.$ vs. $\left.N_{p}\right)$ based on the correlations listed in Table 5.2-1 were plotted for the pitched blade turbine (upward pumping) - anchor coaxial mixer (Figure 5.2-1). All the measurements were conducted at the different speed ratios and the constant gas flow rate of $0.0283 \mathrm{~m}^{3} / \mathrm{min}$. It can be observed that by adopting the correlation suggested by Bao et al. (2011) all power curves obtained at different speed ratios collapsed to one curve; therefore Bao's equivalent rotational speed was used in this study to develop the dimensional and dimensionless gassed power uptake correlations for the coaxial mixer. 
Chapter 5: Results and Discussion

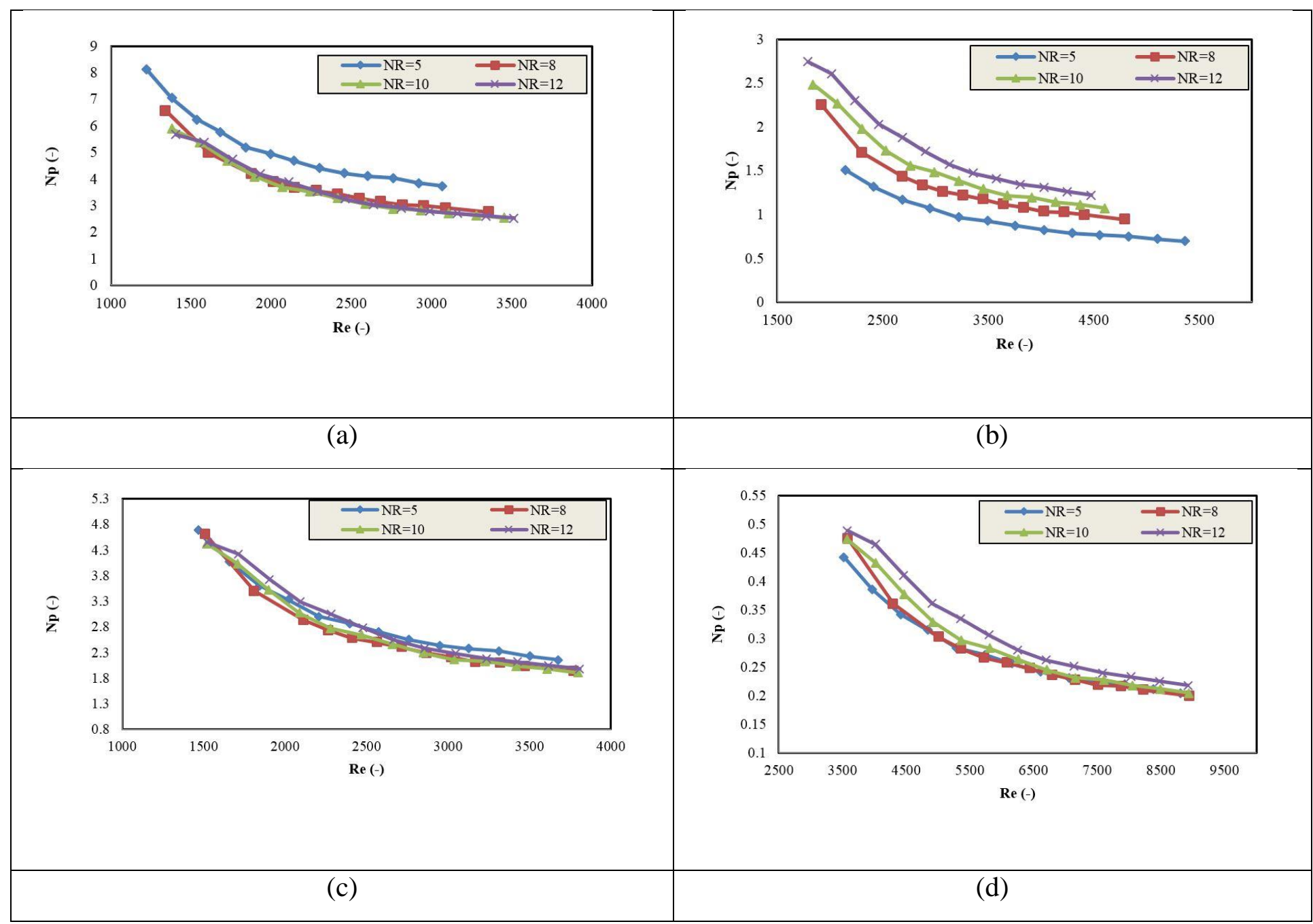

Figure (5.2- 1). Power curve of the PBU-anchor coaxial mixer under constant aeration, $0.0283 \mathrm{~m}^{3} / \mathrm{min}$ , based on different correlations: (a) Foucault et al. (2006), (b) Farhat et al. (2007), (c) Bao et al. (2011), and (d) Liu et al. (2013).

It must be stressed that in the design and scale-up of the aerated agitated tanks, the effect of gas on the impeller power uptake is a critical factor, which should be taken into account by using the dimensionless gas flow number instead of Reynolds number. Therefore, because of the presence of two independently rotating impellers in the aerated coaxial mixing vessel, the development of a novel generalized gas flow number and power number are needed to evaluate the effect of various gas flow rates on the central impeller and anchor power uptakes with the diverse speed ratios. 
Chapter 5: Results and Discussion

Besides, the gassed power $\left(P_{g}\right)$ is defined as an important factor, which reflects the variation in developed flow patterns induced by both impellers and sparged gas. Accordingly, this study was carried out in an attempt to correlate the effect of the central impeller speed, anchor speed, speed ratio, gas flow rate, and viscosity on the power consumption of the coaxial mixers through both dimensional and dimensionless approaches. Based on the data reported on the higher power consumption of the coaxial mixers in the counter-rotating mode of the central impeller and anchor in comparison with that for the co-rotating mode; the performance of the coaxial mixer only in the co-rotating mode was investigated in the current study (Liu et al., 2013; Bao et al., 2011; Farhat et al., 2007; Foucault et al., 2006).

\subsection{Dimensional analysis}

In the open literature, a number of dimensional correlations have been proposed to estimate the aerated power uptake of the single or multi-impeller agitated vessels (Linek et al., 1996; Cui et al., 1996; Abrardi et al., 1990; Michel and Miller, 1962). All the published correlations deal with the aerated systems with a single shaft. As mentioned before, in the current work the employed agitated tank was equipped with two independent impellers which can rotate at various speeds in co/counter-rotating modes. In addition, gas was directly introduced into the tank by means of a ring sparger placed beneath the central impeller. The sparged gas can affect the power uptake of the central impeller and the wall scraping anchor in different ways. In general, the gassed power uptake of the central impeller is less than that of the ungassed systems owing to the existence of the fluid with lower average apparent density in the vicinity of the impeller and enhances streamlined fluid flow past the blades (Dickey, 1979). This is due to the fact that most of freshly sparged gas goes through the centered impeller. Therefore, the central impeller played the main 
Chapter 5: Results and Discussion

role in dispersing the gas throughout the vessel. The aforementioned behavior is in accordance with those reported for the agitators rotating on a common shaft in the aerated tanks (Zhu et al., 2009; Gill et al., 2008). Since the reduction in power consumption under the gassed condition is also related to the impeller type, the pitched blade turbines in upward and downward pumping modes and the up-pumping A310 hydrofoil impeller were considered in this study. It should be mentioned that the A310 impeller was only employed to check the observed results. As it can be seen from Figure 5.2-2, at constant central impeller and anchor speeds, the power consumption of the anchor was increased by increasing the gas flow rates. The possible explanation of this finding can be that in the presence of gas, the power requirement of the central impeller was reduced; leading to a reduction in the dispersion capability of the centered impeller. Therefore, the formation of the stagnant zones in the vicinity of the vessel wall resulted in an increase in the power drawn of the anchor. Hence, the anchor power was affected by the flow pattern induced by the gas and the central agitator. It must be mentioned that, the contribution of the anchor power in the total power uptake of the aerated coaxial mixer was much less than the central impeller power. Accordingly, for the gas dispersing coaxial mixer, the total power was reduced compared to that required for the ungassed system at the same operating conditions. The reason that the PBD data were not presented in Figure 5.2-2 was due to the power fluctuation noticed for this impeller in the presence of gas, which is discussed in details in Section 5.2.2.1.2. 


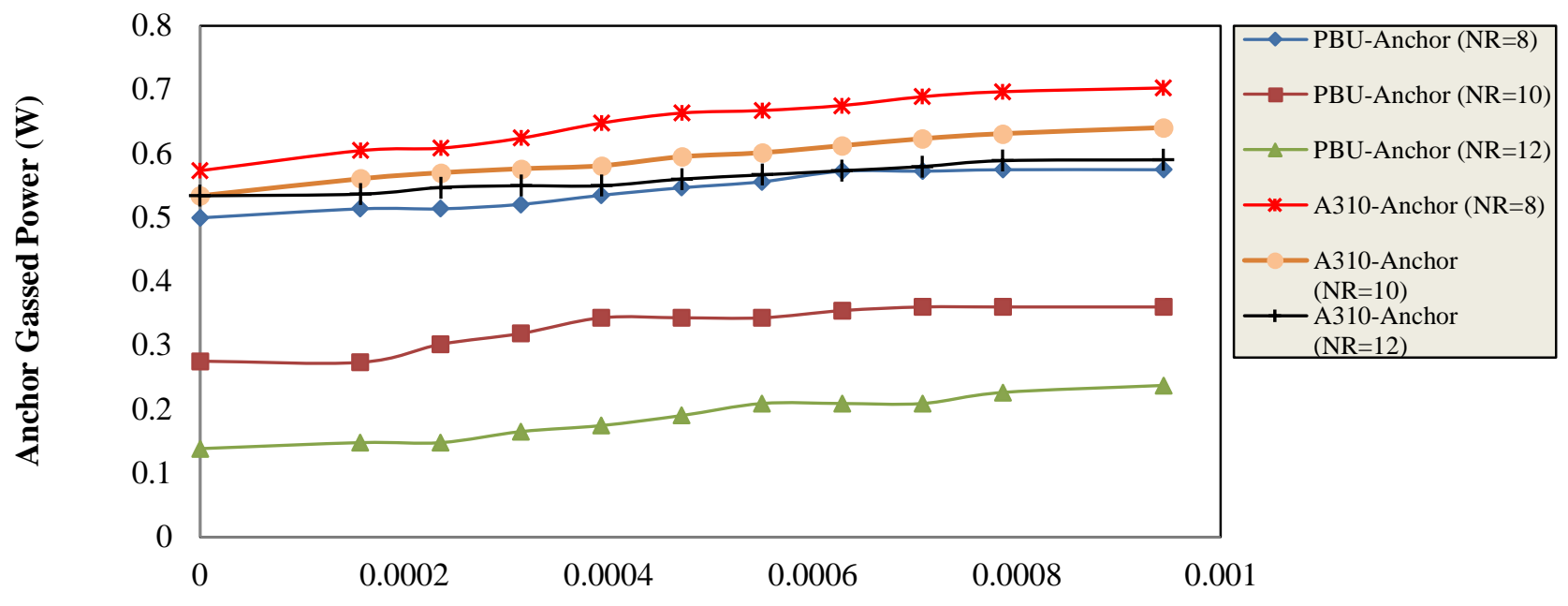

Gas Flow Rate $\left(\mathrm{m}^{3} / \mathrm{s}\right)$

Figure (5.2- 2). Effect of gas on the power consumption of the anchor rotating in the coaxial mixing vessel at $N_{c}=180 \mathrm{rpm}$ and the speed ratios of 8,10 , and 12 .

Michel and Miller (1962) demonstrated that $P_{g} \propto a\left[P_{u g} N D^{b} / Q_{g}^{c}\right]^{d}$. Hence, in the current study, the concept by Michel and Miller was adopted to correlate a novel dimensional equation for the gassed power of the aerated mixing vessel. The obtained experimental results showed that the gassed power $\left(P_{g}\right)$ of the aerated coaxial mixing vessel was dependent on the central impeller type, central impeller speed, anchor speed, speed ratio, gas flow rate and ungassed power uptake of the agitators based on the following relationship:

$$
p_{g}=a\left(\left(N_{c}-\frac{N_{a}}{N_{R}}\right)^{b} \times p_{u g-c}^{c} \times p_{u g-a}^{d} / Q_{g}^{e}\right)^{f}
$$

where $p_{u g-c}, p_{u g-a}$, and $Q_{g}$ are ungassed power of the central impeller, ungassed power of the anchor, and gas flow rate, respectively. 
Chapter 5: Results and Discussion

The regression analysis of the employed coaxial mixer comprised of a central impeller (upward or downward pitched blade turbine) and anchor rotated in the Newtonian corn syrup solutions with the viscosities of 0.05-1.40 Pa.s resulted in the regression constants reported in Table 5.2-2. It should be mentioned that the exponents $c$ and $d$ and the constant $a$ reflect not only the effect of geometrical characteristics of the agitated tank but also the effect of fluid viscosity on the gassed power uptake. The average relative deviation of the results for total gassed power of the coaxial mixing vessel was just slightly above $8 \%$. The excellent fit of the measured and predicted data is shown in Figure 5.2-3. The proposed model can be employed only if the aerated coaxial mixer is operated under the complete dispersion regime.

Table (5.2- 2). Regression constants of the gassed power correlation proposed for the coaxial mixing vessel equipped with PBU-anchor and PBD-anchor.

\begin{tabular}{ccc}
\hline Constant & PBU-Anchor & PBD-Anchor \\
\hline a & 0.9771 & 3.4297 \\
b & 0.5065 & -7.4789 \\
c & 0.3559 & 5.4368 \\
d & 0.1071 & 0.5189 \\
e & 0.0404 & 0.1882 \\
f & 1.4373 & 0.2721 \\
Mean Deviation\% & 14 & 15 \\
\hline
\end{tabular}




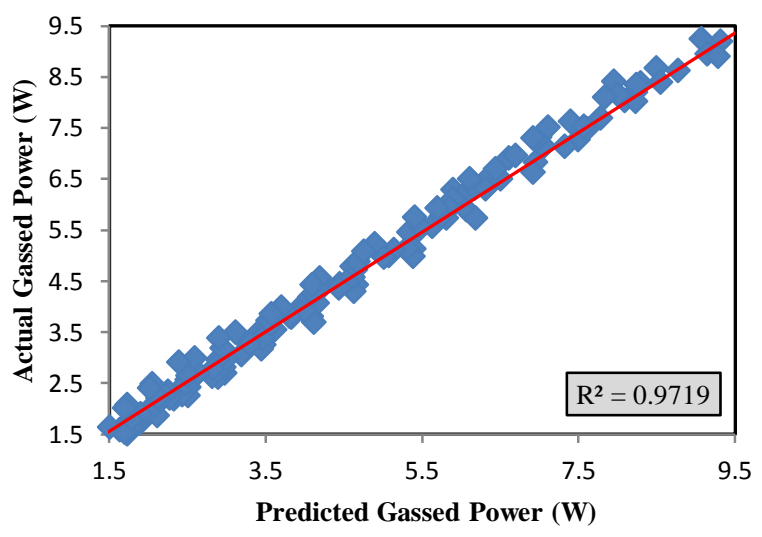

(a)

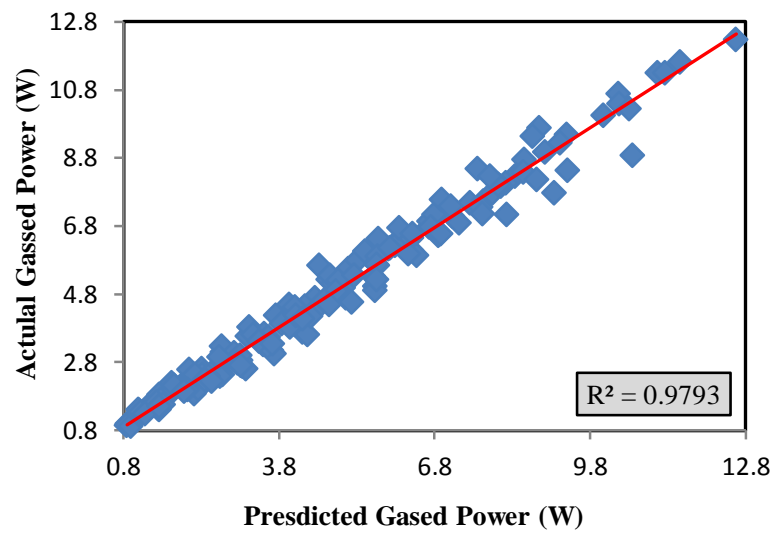

(b)

Figure (5.2- 3). Comparison between the actual total gassed power of coaxial mixers and the predicted ones: (a) PBU-anchor and (b) PBD-anchor.

\subsection{Dimensionless analysis}

The normal practice to evaluate the power consumption of the aerated mixing vessel is to plot the power curve, which is a relation between the gassed power number $\left(N_{P g}\right)$ and the gas flow number $(F l)$. As mentioned before, the coaxial mixers are fitted with two independent motordriven shafts; one is equipped with the central impeller rotating at a high speed, whereas the other shaft is attached to the wall scraping anchor rotating at a low speed. The speed ratio can be defined as the ratio of the centered agitator speed over the anchor speed. Accordingly, four speed ratios of $8,10,12$, and 14 were considered. In addition, the selection of the characteristic speed due to the presence of two independent speeds was done based on the explanations provided in Section 5.2.2.1. The power number was defined using total gassed power uptake of the coaxial mixer, which was the sum of the gassed powers of the centered impeller and the anchor. As can be seen in Figure 5.2-4, the contribution of the central impeller power drawn $\left(P_{g c}\right)$ to the total gassed power 
of the coaxial mixers $\left(P_{g t}\right)$ was much higher than that of the anchor impeller. The reason can be attributed to the higher rotational speed of the central impeller relative to the anchor speed. Besides, from Figure 5.2-4, the gassed power of the PBU dropped as the gas flow rate increased while the PBD showed high torque instabilities in the presence of the gas. This behavior can be attributed to the shape and size of the cavities formed beneath the impeller blades. Figure 5.2-5 shows that the gassed to ungassed power ratio of the central impeller was dependent on the viscosity of the corn syrup solution. The rate of the reduction in power drawn of the central impeller upon aeration increased as viscosity increased. The reason can be that at the higher viscosity the developed cavities behind the impeller blades become more stable. As mentioned before, the decline in the power uptake of the centered impeller affected the anchor power. At a higher viscosity, the anchor power consumption increased due to a greater reduction in the power drawn by the central impeller. In the aerated coaxial mixer, the effect of the speed ratio on the power uptake of the anchor was varied at different fluid viscosity. As can be seen in Figure 5.2$6 \mathrm{a}$, at a relatively low fluid viscosity, the power consumption of the anchor decreased with an increase in the speed ratio. However, at a higher fluid viscosity and beyond the critical speed ratio equal to 10 , the anchor power increased by increasing the speed ratio corresponded to a lower anchor speed (see Figure 5.2-6b). These observations can be related to a decrease in the pumping capacity of the central impeller with respect to the gas flow rate, speed ratio and fluid viscosity. It can be concluded that the influence of speed ratio on the anchor power depends on the combined flow patterns generated by the anchor, central impeller, and gas flow rate. 


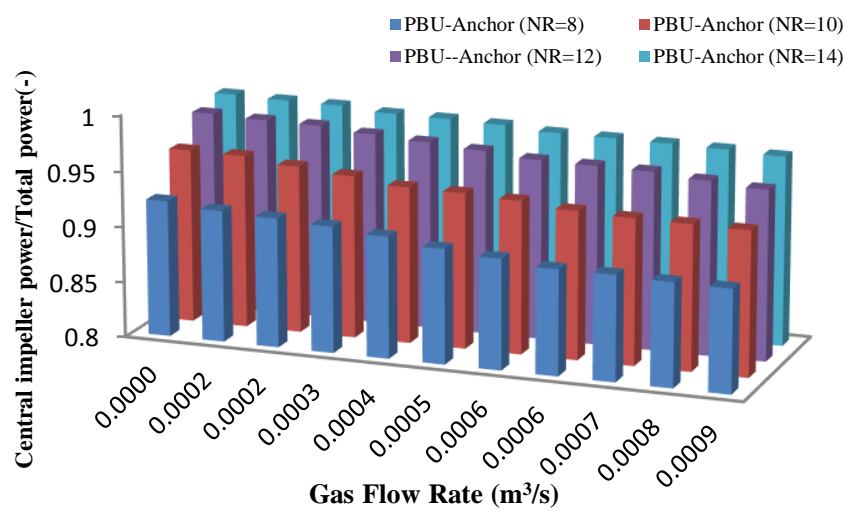

(a)

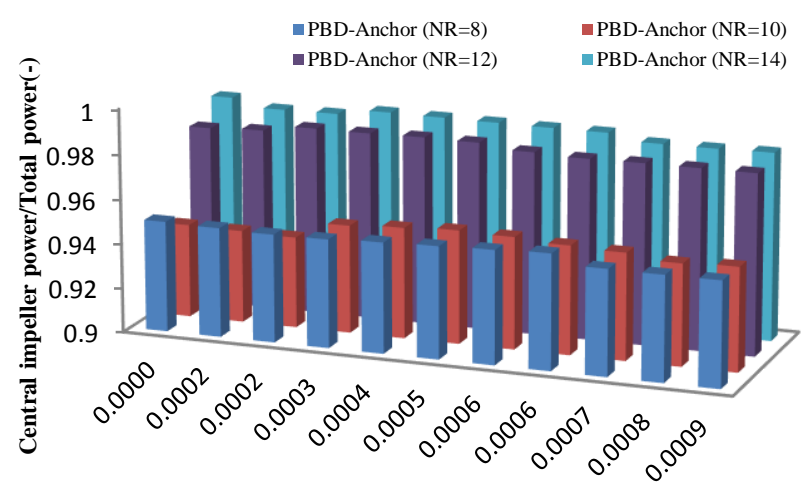

Gas Flow Rate $\left(\mathrm{m}^{3} / \mathrm{s}\right)$

Figure (5.2- 4). Central impeller power contribution to the total gassed power of the aerated coaxial mixer at different speed ratios: (a) PBU-anchor, (b) PBD-anchor.

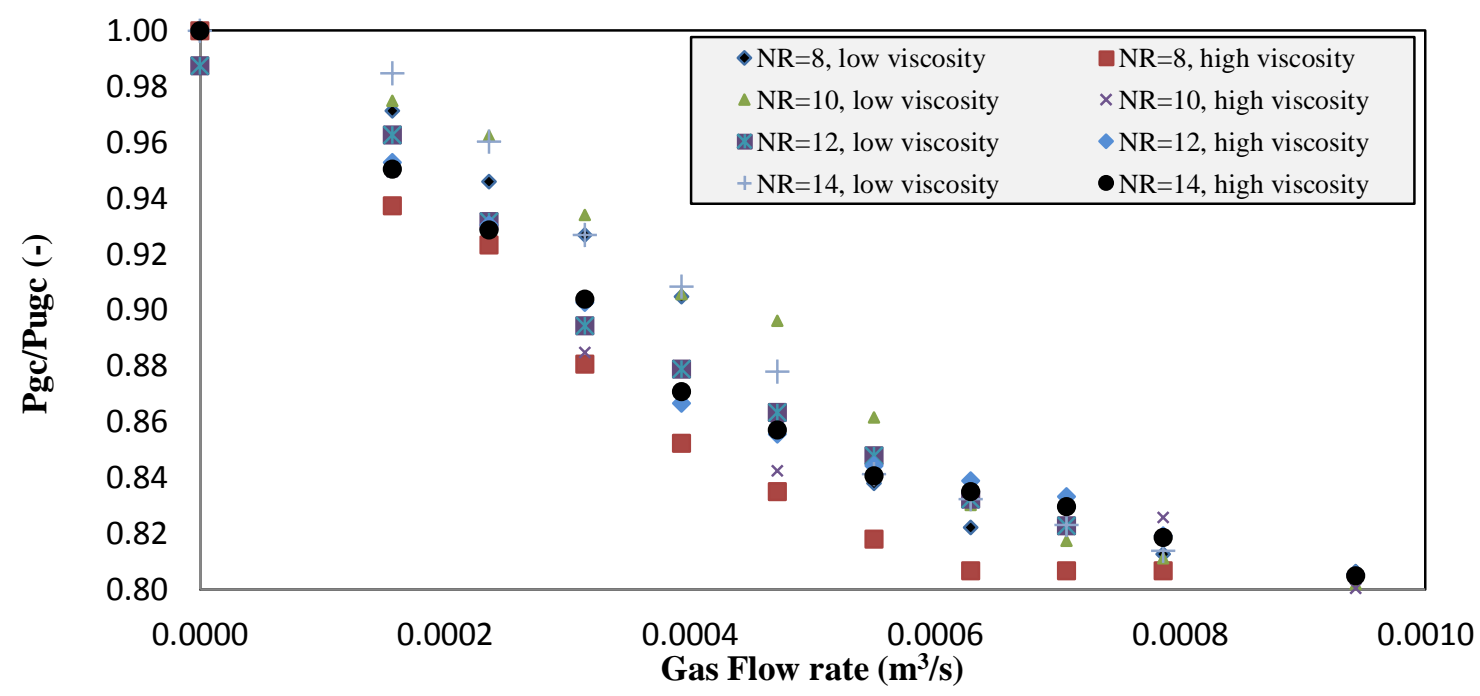

Figure (5.2- 5). Gassed to ungassed power ratio of the PBU impeller used as the central impeller for the coaxial mixer in the agitation of corn syrup solutions with a low viscosity (0.05 Pa.s) and a high viscosity (1.4 Pa.s). 
Chapter 5: Results and Discussion

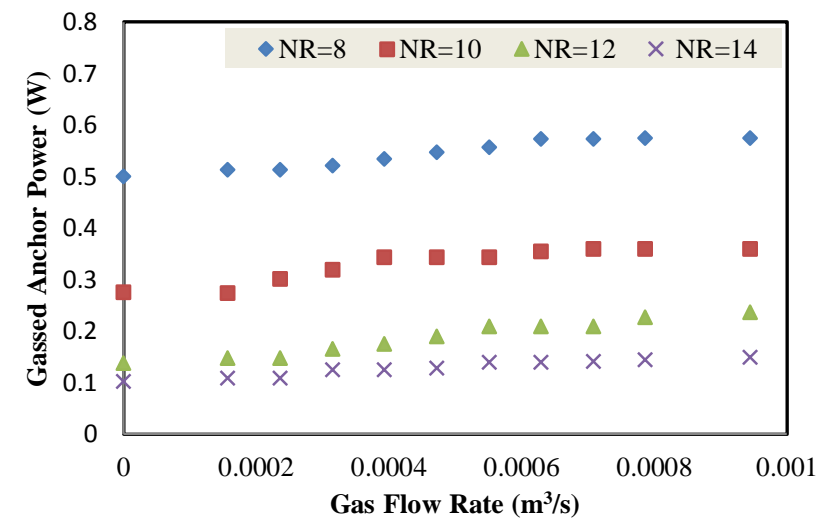

(a)

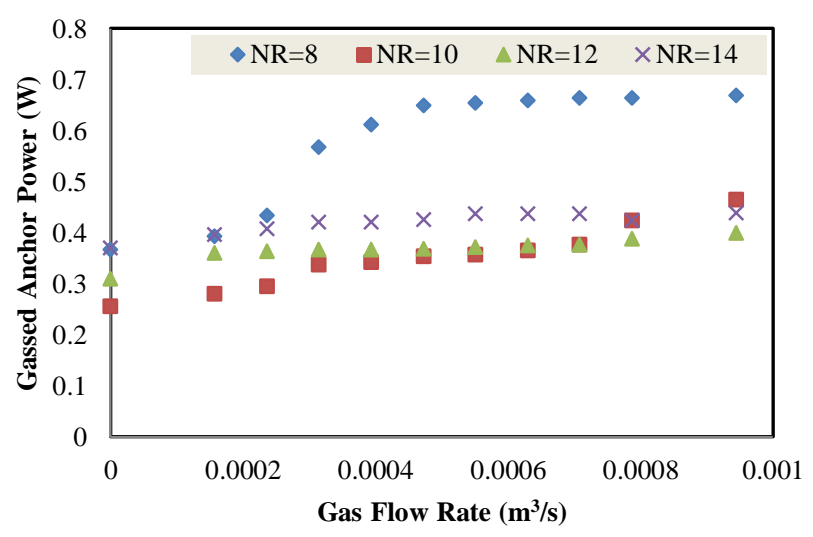

(b)

Figure (5.2- 6). Effect of the speed ratio on the gassed power uptake of the anchor used in a PBUanchor coaxial mixer rotated in the corn syrup solutions with the viscosities of (a) $\mu=0.05 \mathrm{~Pa} . \mathrm{S}$ and (b) $\mu=1.40$ Pa.s.

All observed results guided us to propose a new generalized gas flow number $\left(F l_{g e n}\right)$ and gassed power number $\left(N_{P g}\right)$ to evaluate the hydrodynamics occur in the aerated coaxial mixing vessel. In order to do this, the characteristic speed definition proposed by Bao et al. (2011) was utilized. According to the aforementioned explanations, the contribution of the central impeller power to the total power consumption of the coaxial mixer was much higher than that by the anchor. Therefore, the central impeller power fraction as a function of the speed ratio was incorporated into the new generalized gas flow number and gassed power number as follows:

$$
\begin{gathered}
N_{P g}=\frac{p_{g}}{\rho\left(N_{c}-\frac{N_{a}}{N_{R}}\right)^{3}\left(\frac{1}{p_{c t}{ }^{1 / N_{R}}}\right)^{5} D_{c}^{5}} \\
F l_{g e n}=\frac{Q_{g}}{\left(N_{c}-\frac{N_{a}}{N_{R}}\right)\left(\frac{1}{p_{c t}{ }^{1 / N_{R}}}\right)^{3} D_{c}^{3}}
\end{gathered}
$$


Figure 5.2-7 to 5.2-10 exhibit the gassed power curve ( $N_{P g}$ versus $F l_{g e n}$ ) for the aerated coaxial mixing vessel using Eqs. (5.2-2) and (5.2-3) at various gas flow rates, speed ratios, and fluid viscosities. These proposed correlations were tested by obtained power data for A310-anchor aerated coaxial mixer working with water, Figure 5.2-10. It can be seen that all the depicted power curves at various speed ratios perfectly fell into one curve by employing the novel generalized dimensionless correlations. The only discrepancy was related to the PBD-anchor coaxial mixing vessel at the higher gas flow numbers. This can be attributed to the stable behavior of the upward pumping axial impeller (PBU and A310) upon aeration while the downward pumping one (PBD) was subject to severe undesirable torque and power instabilities especially at higher gas flow numbers. These results showed that the developed dimensionless correlations were able to predict the influence of the operating parameters on the gassed power uptake of the aerated coaxial mixers equipped with an anchor and an axial upward pumping impellers or the downward pumping ones before fluctuations happen. Also, it should be mentioned that these correlations are applicable for loading and complete dispersion gas flow patterns. By comparing Figures 5.2-7 and 5.2-8, it was revealed that the PBD-anchor coaxial mixer consumed higher power relative to the PBU-anchor at the same operating conditions. Based on data reported in the literature regarding the relation between the impeller type and the mixing intensity (Moucha et al., 2003); impeller combinations with lower power numbers show a higher mixing performance. Therefore, PBU-anchor with the lower power number provides better mixing conditions in comparison with the PBD-anchor. As it can be seen from Figures 5.2-7b and 5.2-9, the influence of the gas flow number on the power number is decreased at higher viscosity value, $\mu=1.4 \mathrm{~Pa} . \mathrm{s}$, which is in agreement with the published results on the power uptakes of the single impeller and multiple impeller agitated systems (Markopoulos and Pantuflas, 2001). In Figure 5.2-7, the value of gassed power number, 
Chapter 5: Results and Discussion

$N_{p g}$, approached to a constant value between 0.75 to 0.8 by increasing the gas flow number in the PBU-anchor coaxial mixer working with the low viscous, 0.05 Pa.s, corn syrup solution, however at the higher viscosity, 1.40 Pa.s, and at equivalent operating conditions, $N_{p g}$ did not approach to a constant value, see Figure 5.2-9, corresponding to the transient regime.

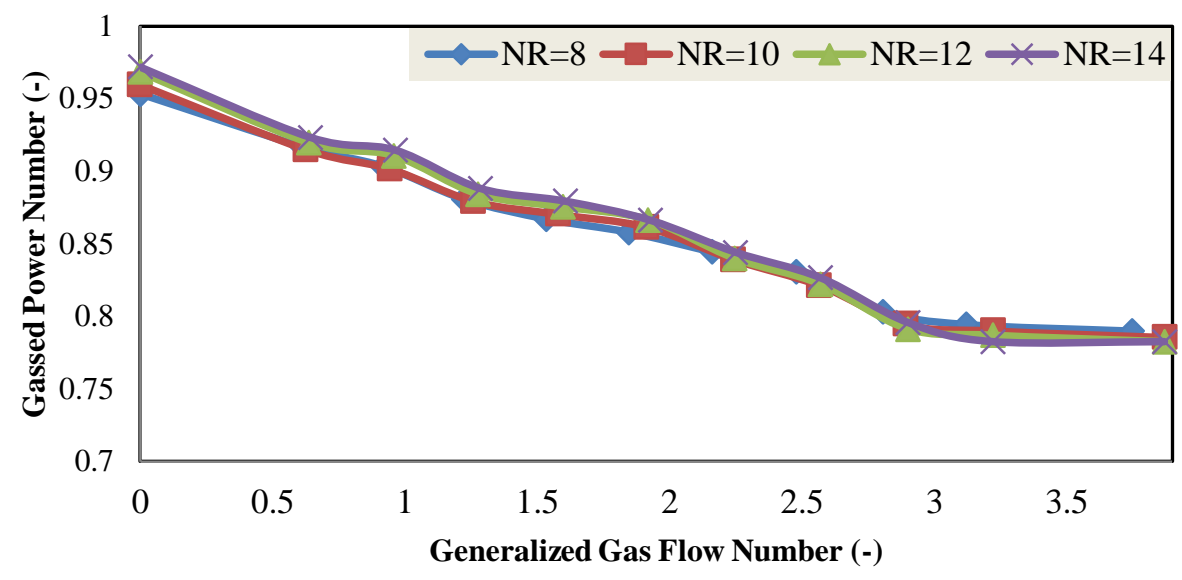

(a)

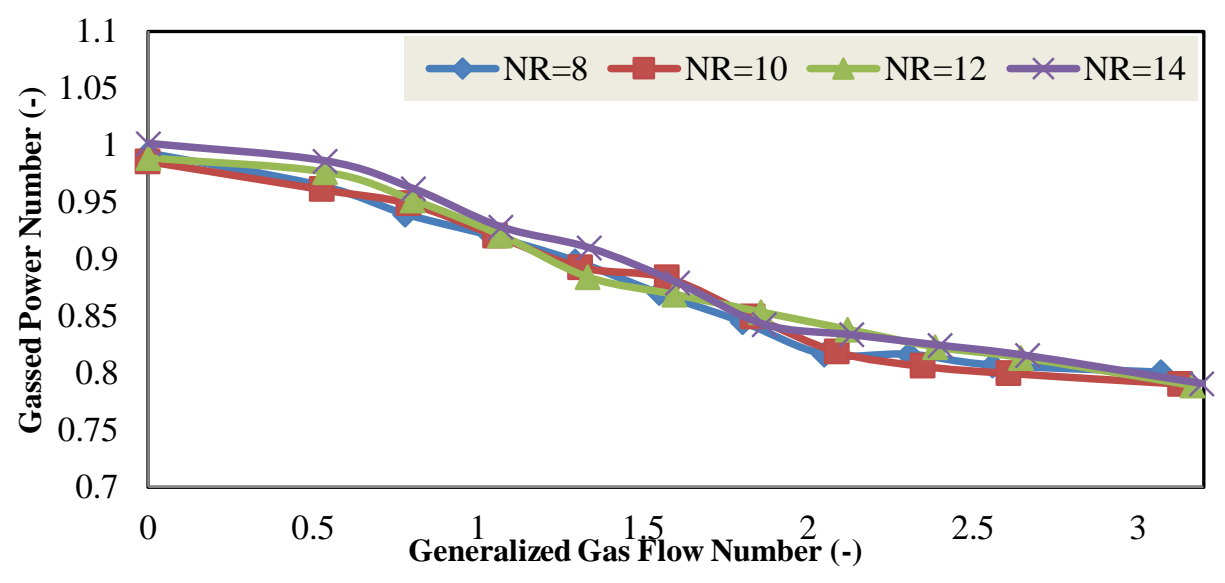

(b)

Figure (5.2- 7). Gassed power curve for the PBU-anchor coaxial mixer at different speed ratios and $\mu=0.05$ Pa.s: (a) $N_{c}=150 \mathrm{rpm}$ and (b) $N_{c}=180 \mathrm{rpm}$. 
Chapter 5: Results and Discussion

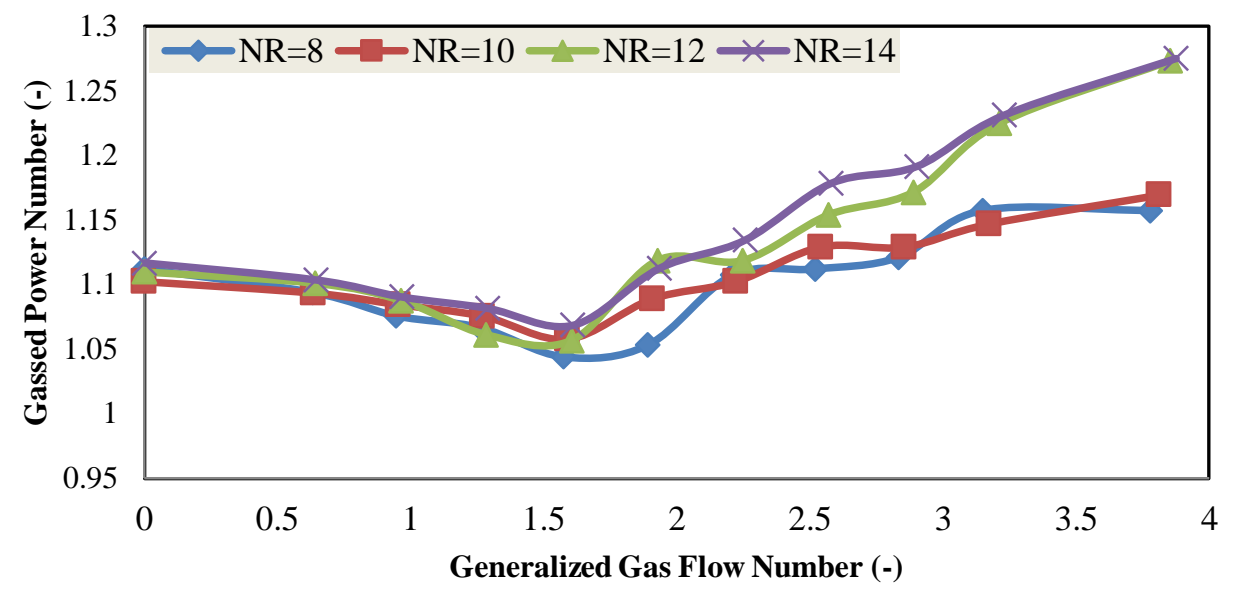

(a)

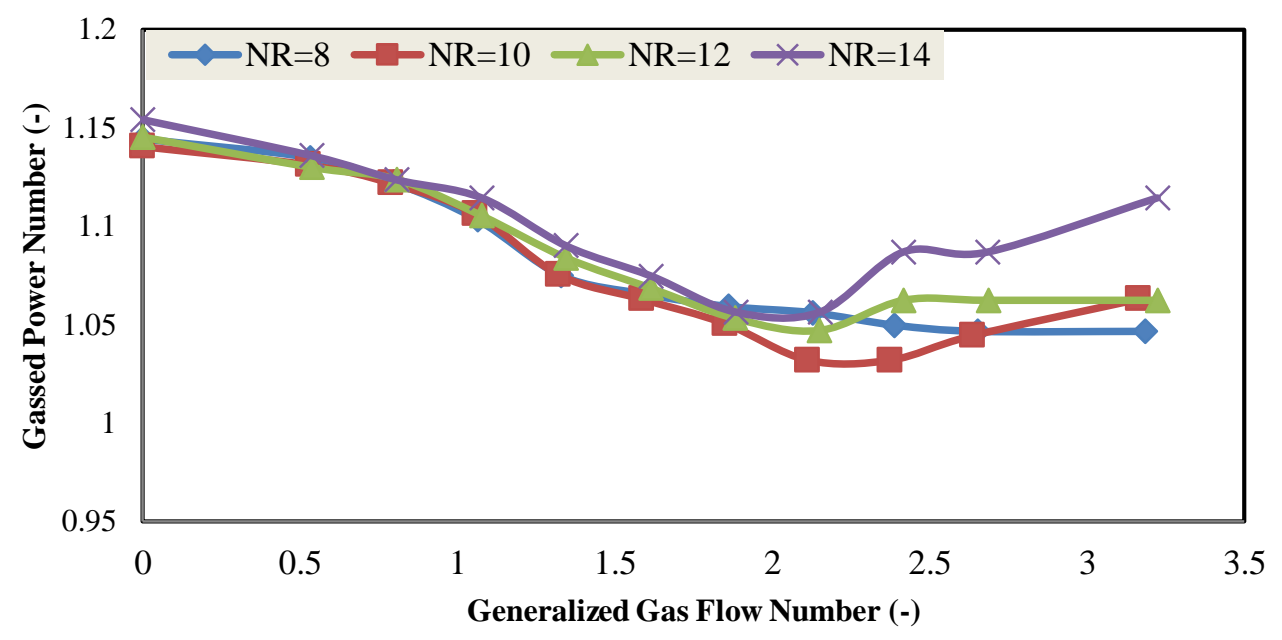

(b)

Figure (5.2- 8). Gassed power curve for the PBD-anchor coaxial mixer at different speed ratios and $\mu=0.05$ Pa.s: (a) $N_{c}=150 \mathrm{rpm}$ and (b) $N_{c}=180 \mathrm{rpm}$. 
Chapter 5: Results and Discussion

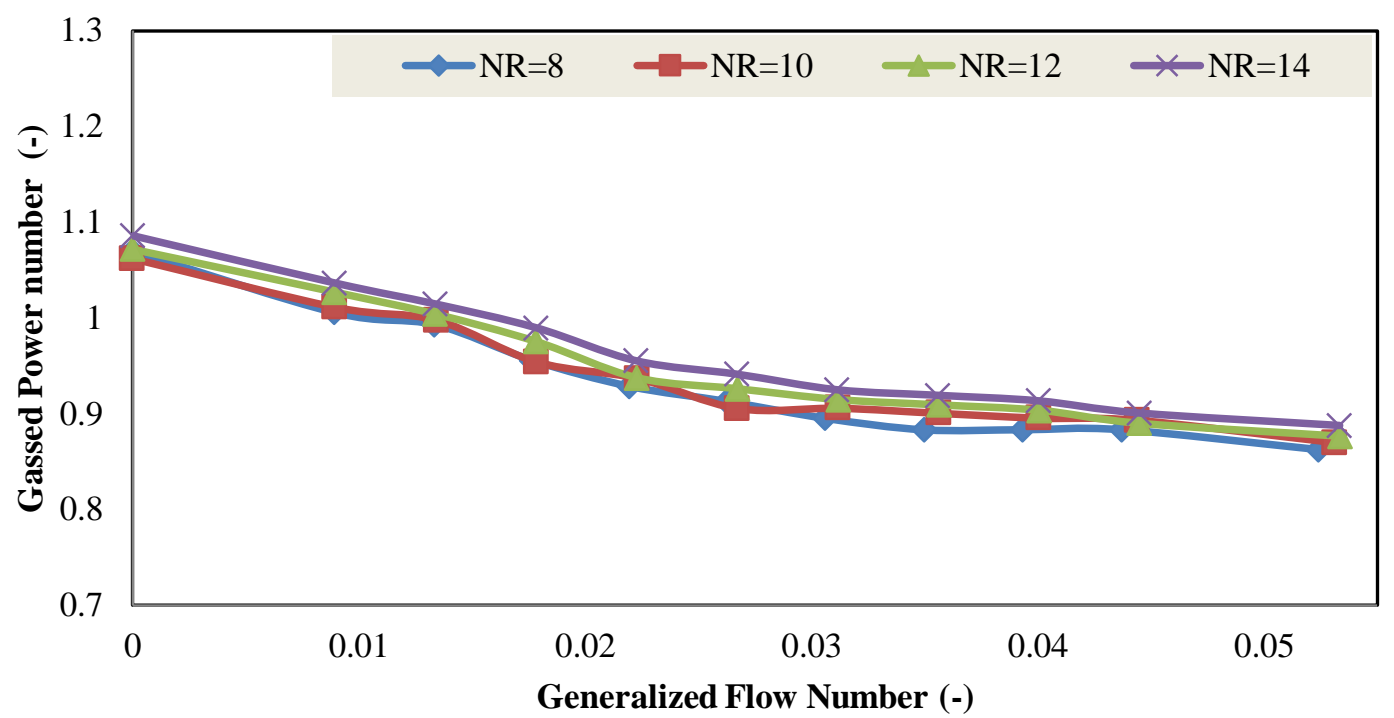

Figure (5.2- 9). Gassed power curve for the PBU-anchor at different speed ratios, $N_{c}=180 \mathrm{rpm}$, and $\mu=1.4$ Pa.s.

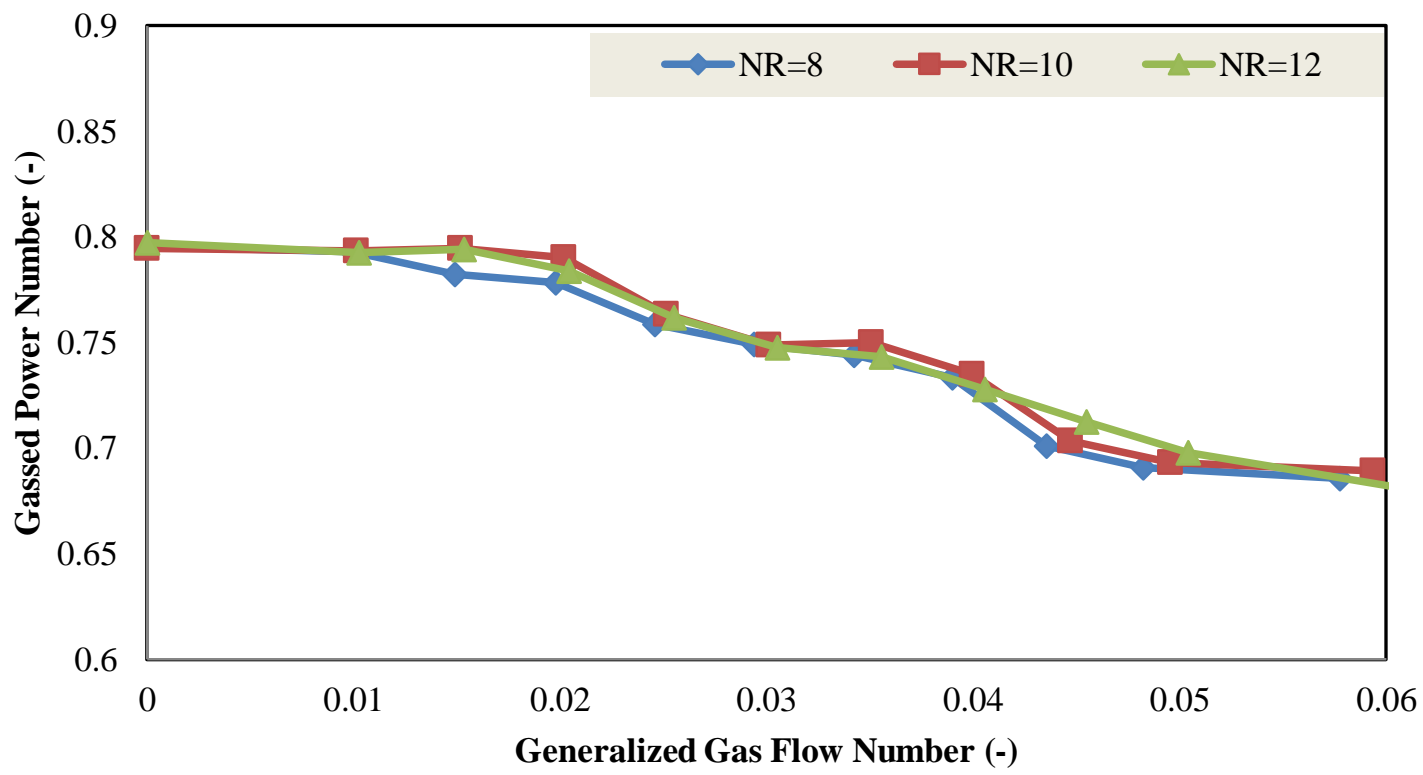

Figure (5.2- 10). Gassed power curve for the A310-anchor coaxial mixer rotated in water at different speed ratios and $N_{c}=150 \mathrm{rpm}$. 
Chapter 5: Results and Discussion

\subsubsection{Radial gas holdup distribution in the aerated coaxial mixing vessel}

As mentioned earlier when the results depicted in Figure 5.2-8 were discussed, the PBD-anchor coaxial mixer was subjected to the torque and power instabilities upon aeration, and the torque oscillations were more significant at the higher gas flow numbers. However, these instabilities in power consumption were not observed for the PBU-anchor coaxial mixer as can be seen in Figure 5.2-7. These results show that the PBU-anchor coaxial mixer is recommended for the gas dispersion. Thus, the radial gas holdup distribution inside the mixing vessel was analyzed only for the PBU-anchor coaxial mixer in this section.

The capability of the ERT system in obtaining the local gas holdup enables us to measure the radial gas holdup distribution at the different fluid heights inside the mixing vessel. To achieve this goal, four tomography sensor planes were considered in this study, see Figure 3.3-4. It must be mentioned that in order to achieve the complete gas dispersion in the mixing vessel, the central impeller speed and the gas flow rate were fixed at $180 \mathrm{rpm}$ and $0.0283 \mathrm{~m}^{3} / \mathrm{min}$, respectively. The anchor speed varied to evaluate the effect of different speed ratios $(18,14,10,9$, and 8$)$ on the radial gas holdup distribution as depicted in Figure 5.2-11. The data presented in Figure 5.2-11 revealed that the distributions of the gas holdup at various speed ratios were uneven in the radial direction. It should be mentioned that uneven average gas holdup values at different planes were related to the fact that only the data for the half of the tank were presented in Figure 5.2-11. In fact, the gas holdup was not the same for both sides of the tank even at the same height. These results also show that the largest gas holdups were captured at Planes 3 and 4 (see Figure 3.3-4 for the location of the tomography planes) near the center of the vessel which was related to the presence of the sparger and developed cavities behind the impeller blades. However, the gas holdup was 
Chapter 5: Results and Discussion

gradually reduced by increasing the height of the vessel and in the vicinity of the top shaft (Planes 1 and 2).

The presence of the gas close to the vessel wall can be attributed to the rotation of the anchor and the zig-zag bubble rise path for the pitched blade turbines, which was reported by Martin et al. (2001). Although the rotation of the anchor played a significant role in dragging the bubbles toward the vessel wall, increasing the anchor speed (i.e. decreasing the speed ratio) did not always result in enhancing the local gas holdup near the wall. For instance, the average gas holdup values between the dimensionless radius (r/R) of 0.7 and 1 were $0.023,0.026,0.030,0.021$, and 0.019 at the speed ratios equal to $18,14,10,9$, and 8 , respectively. Therefore, at speed ratio equal to 10 , the local gas holdup near the vessel wall was maximized (see Figure 5.2-11c). This might be happened due to the combined effects of the developed flow patterns by the central impeller, anchor and the sparged gas. As explained in details in section 5.2.2-3, at the higher fluid viscosity and beyond the critical speed ratio of 10 , the anchor power increased by increasing the speed ratio (i.e. decreasing the anchor speed), which may lead to a lower gas holdup close to the vessel wall. The explanation can be that an anchor with a higher power uptake was capable to drag more bubbles toward the vessel wall, therefore coalescence happened and bubbles with a larger size moved toward the liquid surface rapidly and disappeared. The dependency of the gas holdup on the total gassed power of the coaxial mixer will be discussed in section 5.3. 
Chapter 5: Results and Discussion

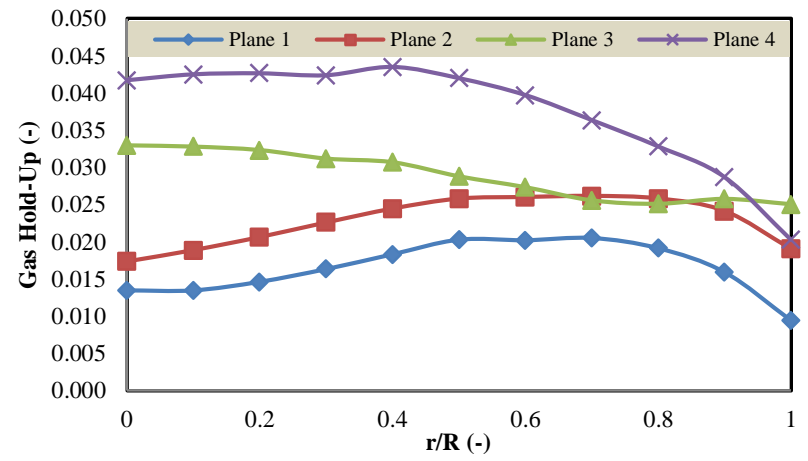

(a)

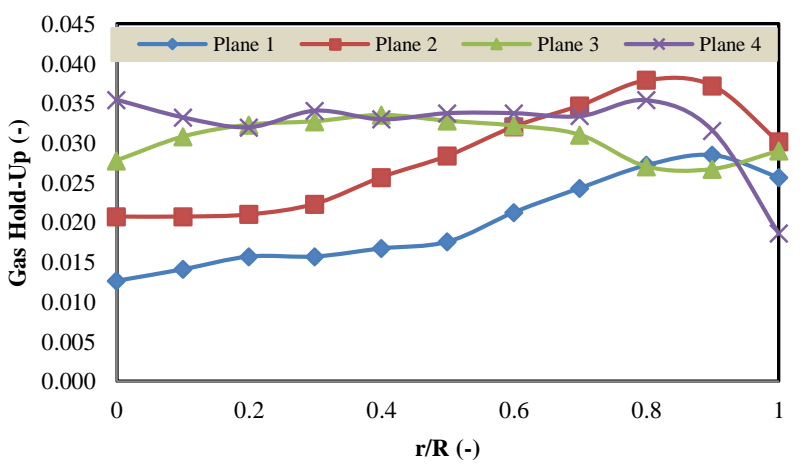

(c)

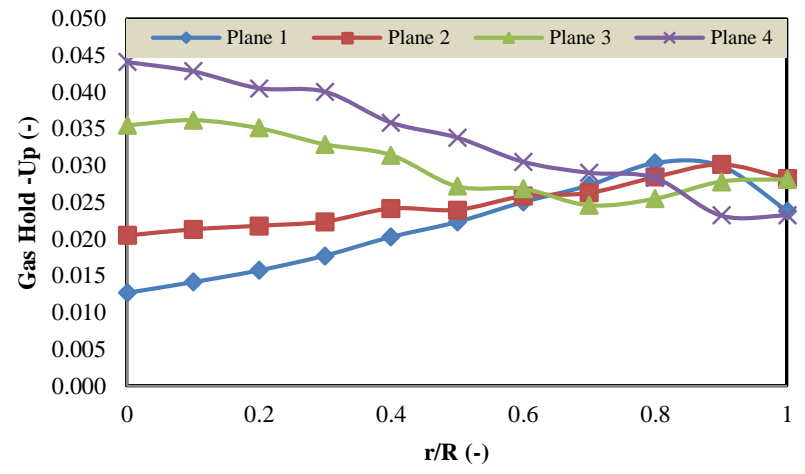

(b)

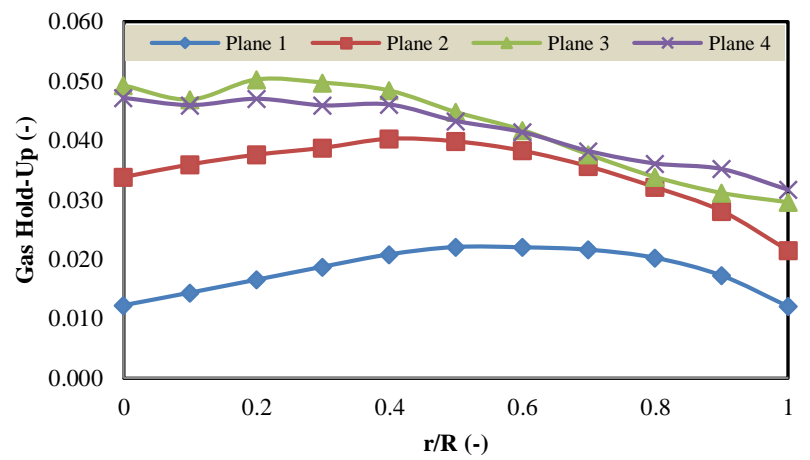

(d)

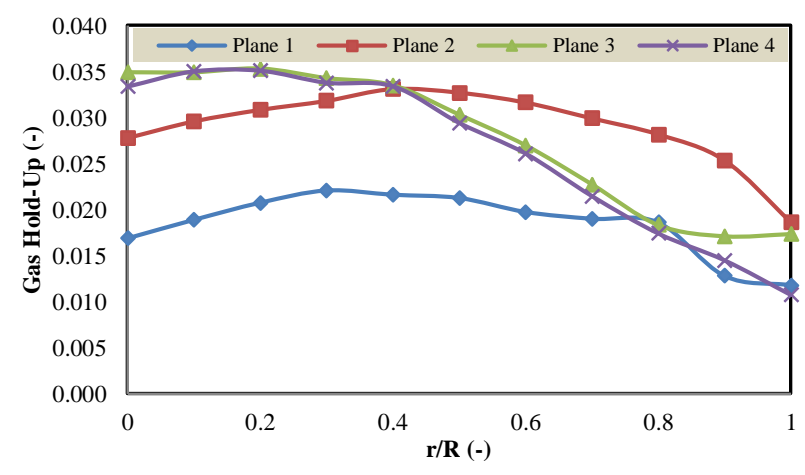

(e)

Figure (5.2- 11). Radial gas distribution attained by the PBU-anchor coaxial mixer at different speed ratios and $\mu=1.4$ Pa.s: (a) $N_{R}=18$, (b) $N_{R}=14$, (c) $N_{R}=10$, (d) $N_{R}=9$, and (e) $N_{R}=8$. 
Chapter 5: Results and Discussion

\subsubsection{Conclusion}

In order to characterize the power consumption of an aerated coaxial mixing vessel consisting of a close clearance anchor and a central impeller, novel correlations for the gas flow number and power number were developed to obtain a master power curve for the aerated coaxial mixing vessel. The experimental results demonstrated that the gassed power of the aerated coaxial mixing $\operatorname{vessel}\left(P_{g}\right)$ was dependent on the central impeller type, central impeller speed, anchor speed, speed ratio, gas flow rate, and the ungassed power uptake of the agitators. Accordingly, a dimensional correlation was proposed for the estimation of the aerated power uptake of the coaxial mixers as a function of the abovementioned design parameters. In addition, it was found that the effect of the sparged gas on the power uptake of the central impeller and the wall scraping anchor was completely different. In general, by increasing the gas flow rate, the gassed power uptake of the central impeller was decreased while the aerated anchor power was increased. Besides, it was demonstrated that the contribution of the central impeller power drawn to the total gassed power of the coaxial mixer was much higher than that for the anchor impeller. The rate of reduction in the central impeller power drawn upon aeration increased as viscosity increased. This resulted in a higher power consumption by the anchor impeller. At a relatively low fluid viscosity, the power consumption of the anchor decreased with an increase in the speed ratio. However, at a higher fluid viscosity and beyond the critical speed ratio equal to 10 , the anchor power increased by increasing the speed ratio (i.e. decreasing the anchor speed). Furthermore, the tomography data revealed that the distributions of the gas holdup at various speed ratios were uneven in the radial direction. Increasing the anchor speed (i.e. decreasing the speed ratio) did not result in enhancing the local gas holdup near the vessel wall. 
Chapter 5: Results and Discussion

\subsection{Experimental investigation of the bubble behavior in an aerated coaxial mixing vessel through electrical resistance tomography (ERT)}

\subsubsection{Introduction}

The main objectives of the current work were to assess the main bubble characteristics including number of bubble size classes, contribution of each class of bubble in the overall gas holdup, bubble size, and gas holdup within the aerated coaxial mixing vessel by means of the dynamic gas disengagement technique (DGD) coupled with ERT. Also a correlation relating the gas holdup to the input power per unit volume, linear gas velocity, and speed ratio (speed of the central impeller over the anchor speed) was developed. It must be stressed out that in this section only the complete dispersion flow pattern was evaluated.

The proposed models for the estimation of the bubble size are usually semi-empirical relations, which have been developed based on the Kolmogoroff's theory that the surface tension and turbulent stresses are balanced. Regarding this theory, Calderbank (1958) employed a critical Weber number to calculate the maximum drop (bubble) size in the gas-liquid agitated vessels, which was governed by the bubble breakup. It was shown that the maximum bubble size was proportional to the Sauter mean bubble diameter. This concept was based on the assumption that at a specified bubble diameter, the disturbing forces are in equilibrium with the stabilizing forces. The disturbing forces were in proportion with the local specific dissipation rate. The stabilizing forces can be expressed as a function of the surface tension and also inversely a function of the bubble size. Therefore, the Sauter mean diameter can be written as:

$$
d_{s}=4.15\left(\frac{\sigma^{0.6}}{\left(P / V_{l}\right)^{0.4} \rho_{l}^{0.2}}\right) \phi_{g}^{0.2}\left(\frac{\mu_{g}}{\mu_{l}}\right)^{0.25}+0.0009
$$


where $\sigma, \mu, \rho, P / V$, and $\phi_{g}$ are defined as surface tension, viscosity of the liquid (1) and gas phase (g), the liquid's density, total aerated power per liquid volume, and gas holdup, respectively. The mean bubble size and the gas holdup are related to the interfacial surface area by following relation:

$$
a=6 \phi_{g} / d_{s}
$$

In order to measure the Sauter bubble size, the gas holdup of the various classes of the bubbles in the DGD process can be calculated using data obtained from the ERT system through Eq. (3.32). The mean size of the bubbles with different classes can be estimated from Eq. (5.3-1) by using the local gas holdup obtained from the tomography results and the power measured from the torque values recorded for the central and anchor impellers. The experimental conditions of this section were summarized in Table 5.3-1.

Table (5.3- 1). The experimental conditions.

\begin{tabular}{cccc}
\hline Central impeller speed $(\mathbf{r p m})$ & Anchor speed (rpm) & Gas flow rate (m³/min) & Corn syrup viscosity (Pa.s) \\
\hline $\mathbf{1 5 0}$ & $0-30$ & $0.0094-0.056$ & 0.050
\end{tabular}

\subsubsection{Results and Discussions}

\subsubsection{Local and overall gas holdup of bubbles with different sizes}

The dynamic gas disengagement technique and the tomography data were used to classify different bubble size distributions within the aerated coaxial mixing vessel at various speed ratios. In all considered cases, both the central impeller speed $(150 \mathrm{rpm})$ and the gas flow rate $(0.0378$ $\mathrm{m}^{3} / \mathrm{min}$ ) were constant while the anchor speed was varied. Under these circumstances, a complete dispersion regime was achieved. As mentioned before, two measuring planes, plane 2 and plane 


\section{Chapter 5: Results and Discussion}

4, which were installed at the heights of $0.258 \mathrm{~m}$ and $0.146 \mathrm{~m}$ of the bottom of the tank, respectively, were used to carry out the DGD technique. For both tomography planes, the samples of the disengagement profiles were presented in Figure 3.3-6. Based on the DGD plots obtained at the height of $0.258 \mathrm{~m}$ of the aerated coaxial mixing vessel (plane 2), three straight lines with various slopes $(M=3)$ were found for all studied speed ratios. This denotes the existence of three different bubble classes including large (LB), small (SB), and very small (VSB) bubbles. However, at the height of $0.146 \mathrm{~m}$ of the vessel, plane 4 , four slope breaks were noticed; corresponding to the four types of the bubble classes; large (LB), medium (MB), small (SB), and very small bubbles (VSB). Figure 5.3-1 shows the percentage of the overall gas holdup occupied by each class of bubble as a function of the speed ratio. As it can be seen, the speed ratio did not have an effect on the number of the bubble classes existed in the predefined liquid levels of the aerated coaxial mixing vessel. However, the gas holdup related to each class of bubble was a function of the speed ratio. For both given heights, the larger bubbles occupied a larger volume of the tank. Therefore, it can be concluded that the smaller the size of the bubble, the smaller is the gas holdup at a fixed speed ratio in the aerated coaxial mixing vessel. The impact of the speed ratio on the gas holdup of the existing bubble classes depends on the flow pattern generated by the aerated coaxial mixing tank. The results showed that above the impeller (plane 2) and at all considered speed ratios; the gas holdup variations of the large and small bubble classes were more significant. Figure 5.3-1a demonstrates that the gas holdup of the large bubbles decreased by decreasing the speed ratio (i.e. increasing the anchor speed). The possible explanation could be related to the bubble breakage, which took place either by flow or direct impact of the anchor blades. In fact, at a fixed central impeller speed, the breakup of the bubbles increased with an increase in the anchor speed due to the higher power input of the anchor. This resulted in the reduction of the gas holdup of the largest 
Chapter 5: Results and Discussion

bubble classes. The similar results were observed below the impeller (plane 4); at which the speed ratio was inversely proportional to volume fraction of the bubbles with the largest size due to the breakup process. The largest bubbles were broken into the medium bubble sizes and resulted in a decrease in the gas holdup of the largest bubble sizes. The lower number of the bubble classes in plane 2 (above the impeller) in comparison with plane 4 (below the impeller) was related to the coalescence of the bubbles when traveled upward.

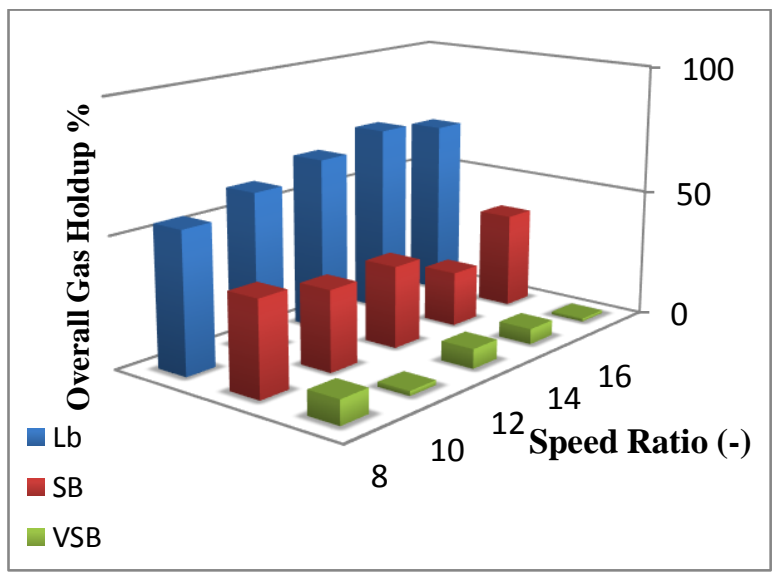

(a) Above the impeller (plane 2)

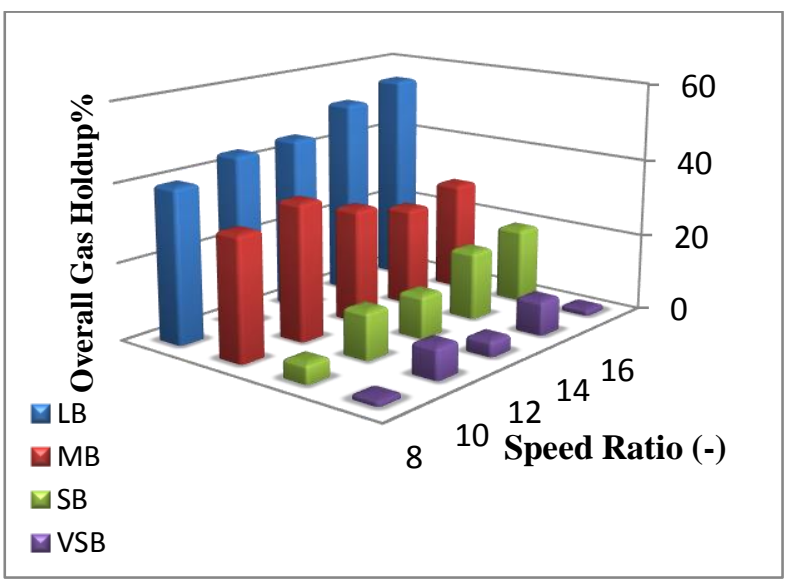

(b) Beneath the impeller (plane4)

Figure (5.3- 1). Contribution of each class of bubble to the overall gas holdup measured using the tomography data obtained from (a) plane 2 and (b) plane 4.

Figure 5.3-2 depicts the local gas holdup of all classes of bubbles, which is a critical factor in the design and scale-up of the aerated coaxial agitated vessel, as a function of the speed ratio. The local gas holdup notably depends on the measurement position and this is in agreement with the observations of Takahashi and Nienow (1993). Bubbles developed under stirring can be affected by the flow induced through the impellers and turbulent eddies. Therefore, the largest local gas holdups (values in red color in Figure 5.3-2) at all speed ratios were obtained in the radial discharge 


\section{Chapter 5: Results and Discussion}

of the central impeller and in the low pressure zone beneath the impeller blades, at which the gas cavities are formed (Riet and Smith, 1975). In addition, the gas holdup in the vicinity of the vessel wall can be related to the zig-zag bubble rise path for the pitched blade turbines, which was suggested by Martin et al. (2008), and the rotation of the anchor impeller. The data presented in Figure 5.3-2 revealed that the local gas holdups near the vessel wall were mostly increased as the speed ratio increased up to the critical value of 10 . However, beyond this critical value, increasing the speed ratio affected the local gas holdup negatively. Therefore, the speed ratio of 10 , corresponding to the rotational speeds of $15 \mathrm{rpm}$ and $150 \mathrm{rpm}$ for the anchor and the central impeller, led to a slight increase in holdups captured in the vicinity of the vessel wall. It can be concluded that the influence of speed ratio on the local gas holdup depends on the combined flow patterns generated by both the anchor and the central impellers. According to the data presented in Figure 5.3-2, it was found that near the top shaft the gas holdup enhanced with increasing the fluid height. As the bubbles spread out, coalescence happened and bubble with the larger sizes caused the bubble rise velocity to increased leading to lower gas holdup. 
Chapter 5: Results and Discussion

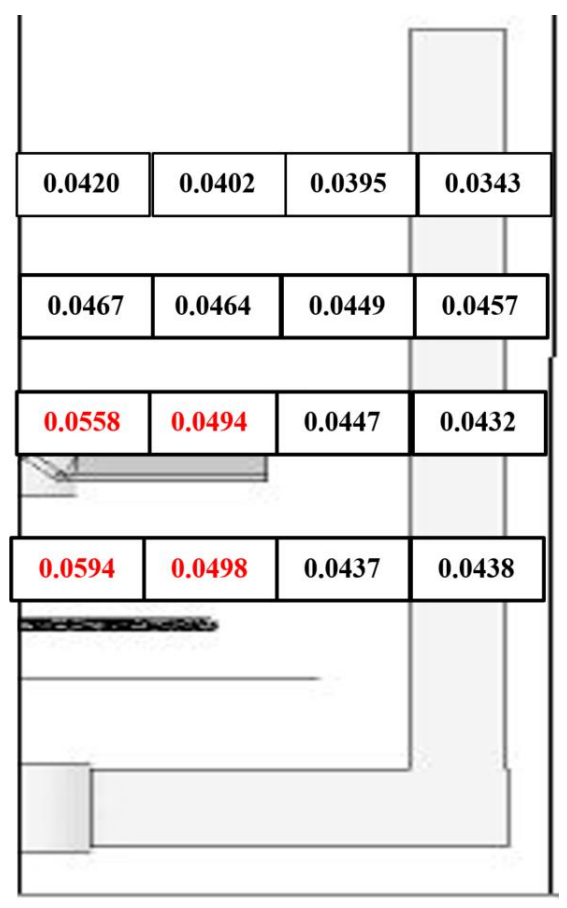

(a)

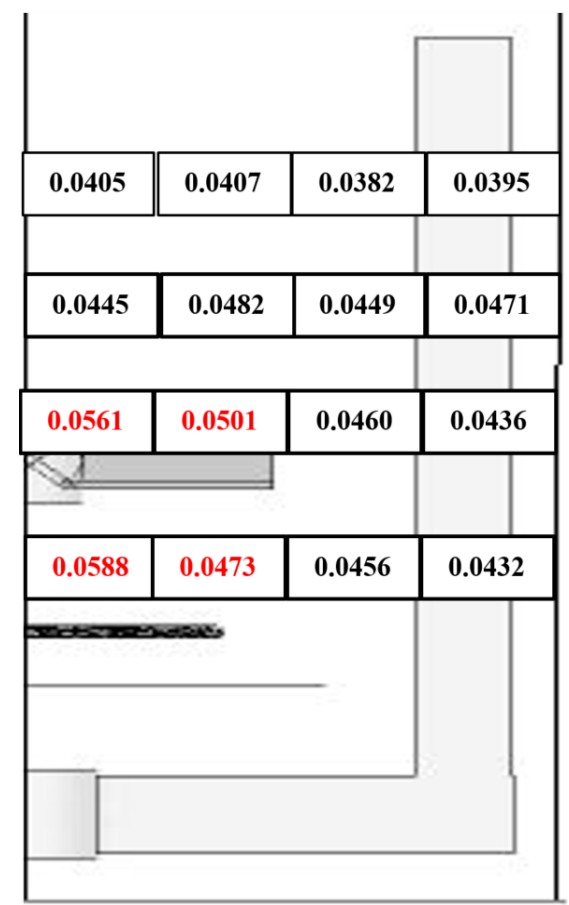

(b)

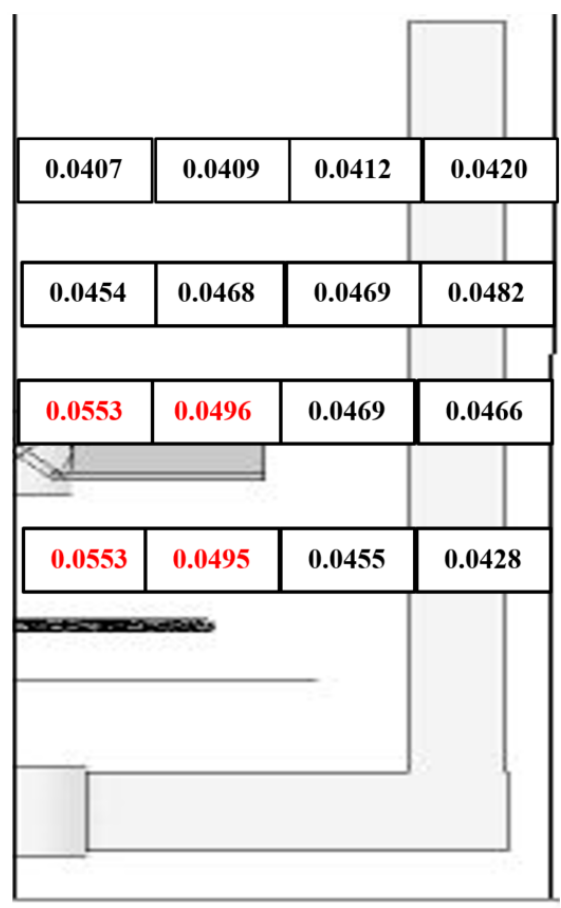

(c)

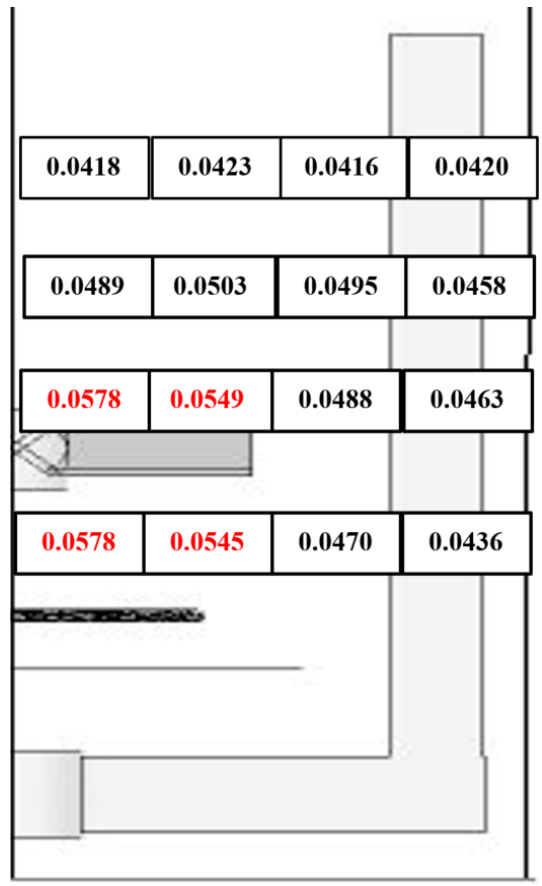

(d)

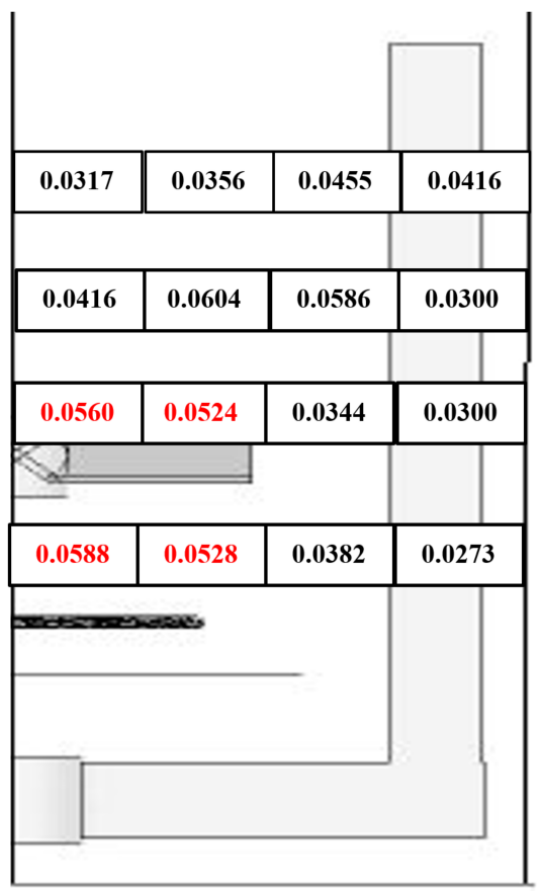

(e)

Figure (5.3- 2). Local gas holdup inside the aerated coaxial mixing vessel at the central impeller speed of $150 \mathrm{rpm}$ and the gas velocity of $0.15 \mathrm{~m} / \mathrm{s}$, and different speed ratios: (a) $N_{R}=14$, (b) $N_{R}=12$, (c)

$$
N_{R}=10, \text { (d) } N_{R}=8 \text {, and (e) } N_{R}=5 \text {. }
$$




\section{Chapter 5: Results and Discussion}

In Figure 5.3-3, the volume averaged gas holdups inside the aerated coaxial mixing vessel as a function of the superficial gas velocity and the speed ratio are exhibited. The obtained data followed the expected trend in terms of gas velocity. The higher the superficial gas velocity, the more bubbles existed in the agitated vessel. However, the effect of the speed ratio was different in comparison with that obtained for the local gas holdup near the vessel wall. As it can be observed from Figure 5.3-3, the lowest overall gas holdup values corresponded to the speed ratio equal to 10 regardless of the gas velocity. The possible explanation may be that at the speed ratio of 10 , because of the combined flow patterns generated by two coaxial impellers, bubbles dragged more from the sparger toward the anchor blades, resulting in higher local gas holdup near the vessel wall. Therefore, at the critical speed ratio $\left(\mathrm{N}_{\mathrm{R}}=10\right)$ bubble coalescence was more dominant compared with the bubble breakage and forced the bubbles to coalesce and vertically dissipated into the liquid surface; leading to a reduction in overall gas holdup. Additionally, it can be concluded that there was an inverse relation between the local gas holdup close to the vessel wall and the overall gas holdup in the aerated coaxial vessels. 


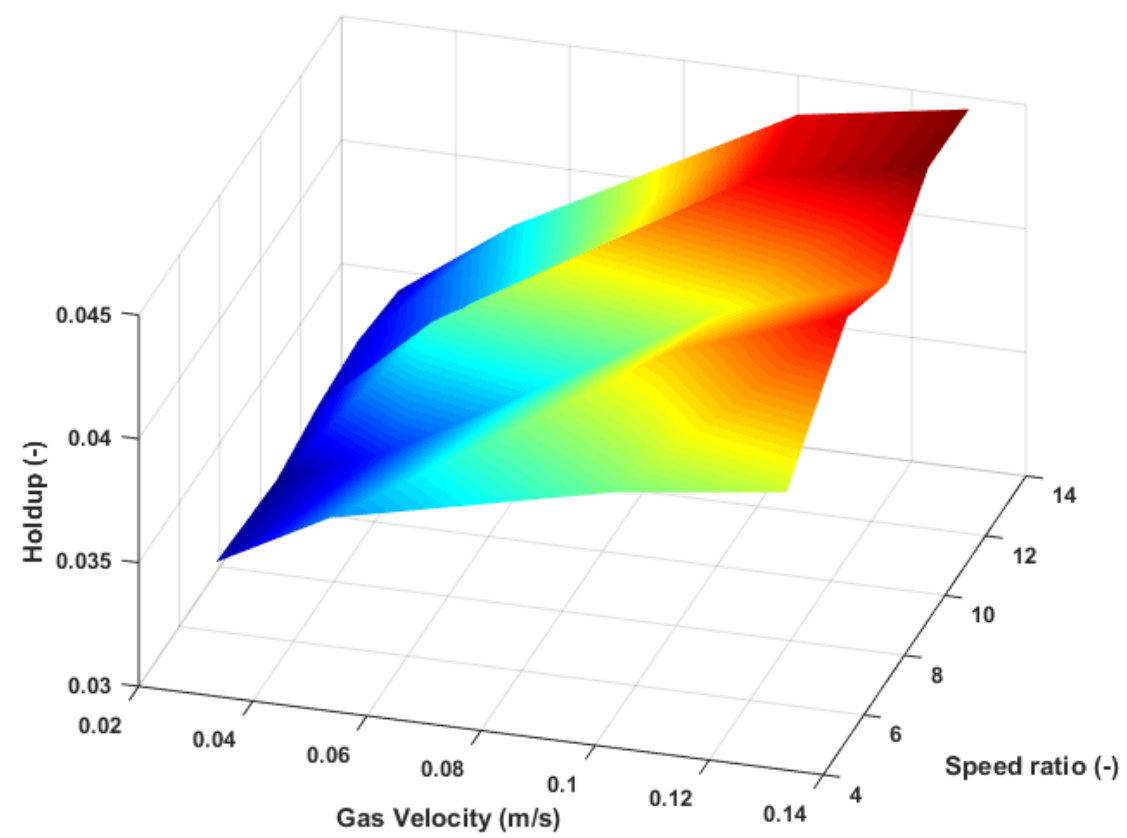

Figure (5.3- 3). Overall gas holdup as a function of gas velocity and speed ratio.

\subsubsection{Gas holdup correlation for the aerated coaxial mixing vessel}

A number of correlations have been suggested in the literature to estimate the gas holdup in the vessel equipped with the single and multiple impellers, and for both Newtonian and nonNewtonian fluids (Vasconcelos, et al., 2000; Pinelli et al., 1994; Nocentini et al., 1993; Meister et al., 1979; Loiseau et al., 1977). Usually gas holdup values are estimated by the following relation:

$$
\varepsilon_{g}=a\left(P / V_{l}\right)^{b} u_{g}^{c} \mu^{d}
$$

Other reported correlations substituted the power input per liquid volume, $P / V_{l}$, by the agitator speed, $N$. As it can be seen in Table 5.3-2, the exponent values are considerably different in published works. This can be related to the vessel geometry, impeller type, number of the impellers, gas sparger type, and furthermore to the fluid properties (density, viscosity, and surface 
Chapter 5: Results and Discussion

tension). Thus, it can be expressed that there is no generalized gas holdup correlation, which can be employed for various geometry and operating parameters.

Table (5.3- 2). Different published correlations for the prediction of gas holdup in agitated vessels

\begin{tabular}{ll}
\hline Authors & Correlation \\
\hline Louiseau et al. (1977) & $\phi_{g}=\left(P / V_{l}\right)^{0.3} u_{g}^{0.4} \mu^{-0.1}$ \\
Meister et al. (1979) & $\phi_{g}=N^{0.6} u_{g}^{0.4}$ \\
Nocentini et al. (1993) & $\phi_{g}=\left(P / V_{l}\right)^{0.375} u_{g}^{0.62}$ \\
Pinelli et al. (1994) & $\phi_{g}=\left(P / V_{l}\right)^{0.2} u_{g}^{0.6}$ \\
Vasconcelos et al. (2000) & $\phi_{g}=\left(P / V_{l}\right)^{0.37} u_{g}^{0.65}$ \\
\hline
\end{tabular}

After considering previous published papers dealing with the estimation of the overall gas holdup in the agitated tanks, it was noticed that no correlation has been proposed for the assessment of the gas holdup in the aerated coaxial mixing vessels. Roustan (1985) suggested that the gas holdup can be affected by the number of impellers. As mentioned before, coaxial agitated tanks are fitted with two independent impellers, which can rotate at different speeds at the same or opposite directions. Therefore, the speed ratio of the central impeller and the wall scraping anchor can affect the overall gas holdup along with the other affecting parameters including power per unit volume and superficial gas velocity. Figure 5.3-3 shows the influence of the speed ratio and gas velocity on the gas holdup in the aerated coaxial vessel. In this case, increasing the speed ratio was related to decreasing the anchor speed at constant central impeller speed. Therefore, the experimental measurements in terms of speed ratio, gas velocity, and total gassed power per unit volume were correlated according to the following equation:

$$
\phi_{g}=0.0056\left(N_{R}\right)^{0.328}\left(P / V_{l}\right)^{0.420} u_{g}^{0.220}
$$


where $N_{R}, P / V_{l}$, and $u_{g}$ are the speed ratio, power per unit volume, and superficial gas velocity, respectively. It should be mentioned that the excel solver was used to determine the coefficients of the developed correlation by minimizing the sum of the squares of the differences between the gas holdup values obtained from ERT and the predicted ones. The average relative deviation of the results for gas holdup was just slightly above 3 percent. The excellent fit of the measured and predicted data is shown in Figure 5.3-4. The current model can be employed only if the aerated coaxial mixing vessel works in the complete dispersion regime.

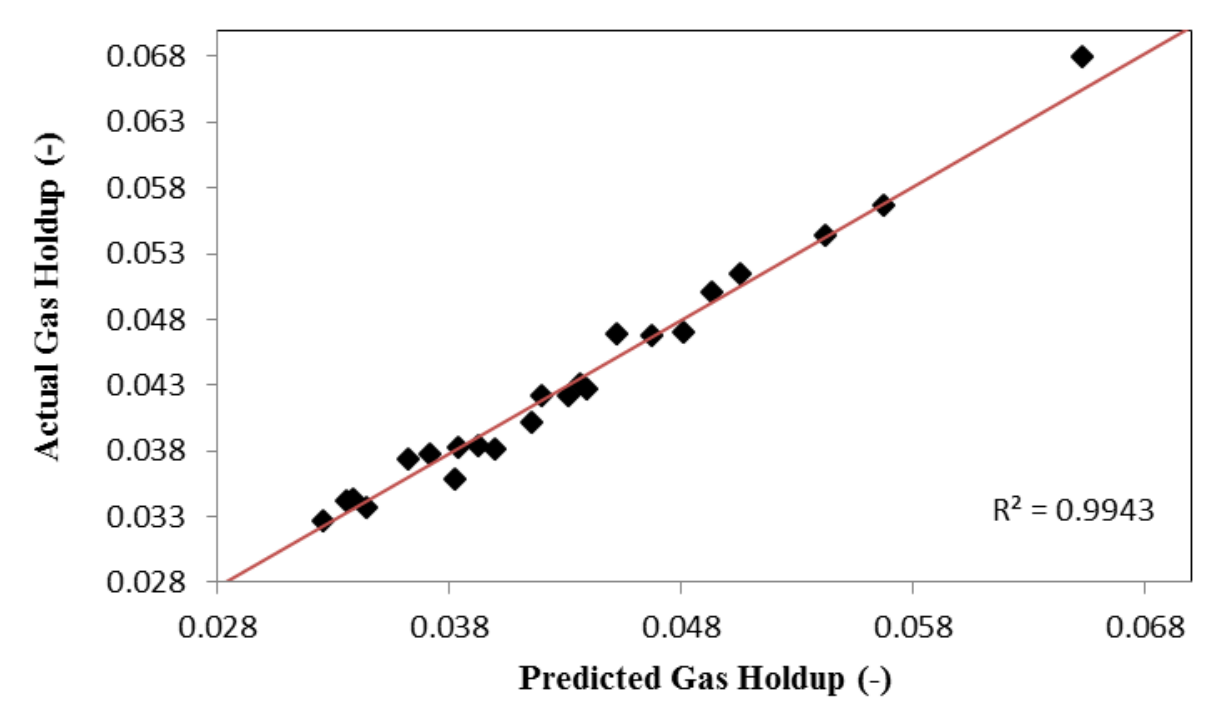

Figure (5.3- 4). Comparison between the experimental gas holdup values and the predicted ones.

\subsubsection{Effect of the speed ratio on the Sauter mean bubble diameter in the aerated coaxial mixing vessel}

The proposed methods in the literature for estimating the bubble sizes are often the semiempirical relations; at which the mean bubble sizes defined based on the equilibrium between surface tension and turbulent stresses using the Kolmogoroff's theory (Calderbank, 1958). In the 
Chapter 5: Results and Discussion

current work, the reputable correlation suggested by Calderbank (1959), Eq. (5.3-1), was used to calculate the mean bubble size in the aerated coaxial mixing vessel. This correlation was given based on the experiments conducted in the stirred vessel equipped with 5 baffles and Rushton turbine working with 10 different liquids. The gas holdup was measured by means of the sampling dispersion to an evacuated glass bulb. Alves et al. (2002) reported that the variation in bubble sizes was not related to the type and number of agitators, size of the vessel, and measuring method. Therefore, Eq. (5.3-1) can be applicable for the aerated coaxial mixing vessel composed of the pitched blade turbine and anchor.

The Sauter mean bubble size versus the speed ratio of different classes of bubbles, which were noticed at two different heights (planes 2 and 4) from the bottom of the aerated coaxial mixing vessel, are shown in Figure 5.3-5. The substituted gas holdup values in Eq. (5.3-1) for different class of bubbles obtained by Eq. (3.3-2) using the acquired tomography data.

As can be concluded from Figure 5.3-5, the bubble sizes were varied by changing the speed ratio and the largest bubbles were observed at the higher speed ratios. As mentioned before, in all conducted experiments the central impeller speed was constant and therefore the higher speed ratio corresponded to the lower anchor speed. Assuming that at the two sensor planes (2 and 4), the mean bubble size of all classes of bubbles varied between 2 to $3.5 \mathrm{~mm}$. Besides, as it can be seen from Figure 5.3-6, the mean bubble sizes of different classes of bubbles at the height equal to the central impeller clearance $(180 \mathrm{~mm})$ changed from 0.8 to $1.2 \mathrm{~mm}$. By comparing the data presented in Figures 5.3-5 and 5.3-6, it was revealed that the smallest bubble sizes appeared at the central impeller level. The explanation can be that the large bubbles, which were sparged in the measurement position (plane 4), traveled upward and when the bubbles reached to the impeller 
Chapter 5: Results and Discussion

blades (plane 3), breakage occurred, leading to the smallest size of the bubbles. The bubble breakup was also occurred due to the rotation of the anchor impeller. The bubble breakup was proportional to the anchor rotational speed as presented in Figure 5.3-6. The bubble coalescence happened as the bubbles moved far from the central agitator and resulted in the larger bubble sizes.

Schafer et al. (2000) observed the larger bubbles in the agitator discharge, which was in disagreement with the results obtained in the current study (see Figure 5.3-6). The aforementioned variation can be attributed to the presence of the anchor close to the vessel wall and interaction between the bubbles and the anchor blades. As a consequence, most of the discharged bubbles from the central impeller traveled to the anchor zone and exposed to the breakup process. 

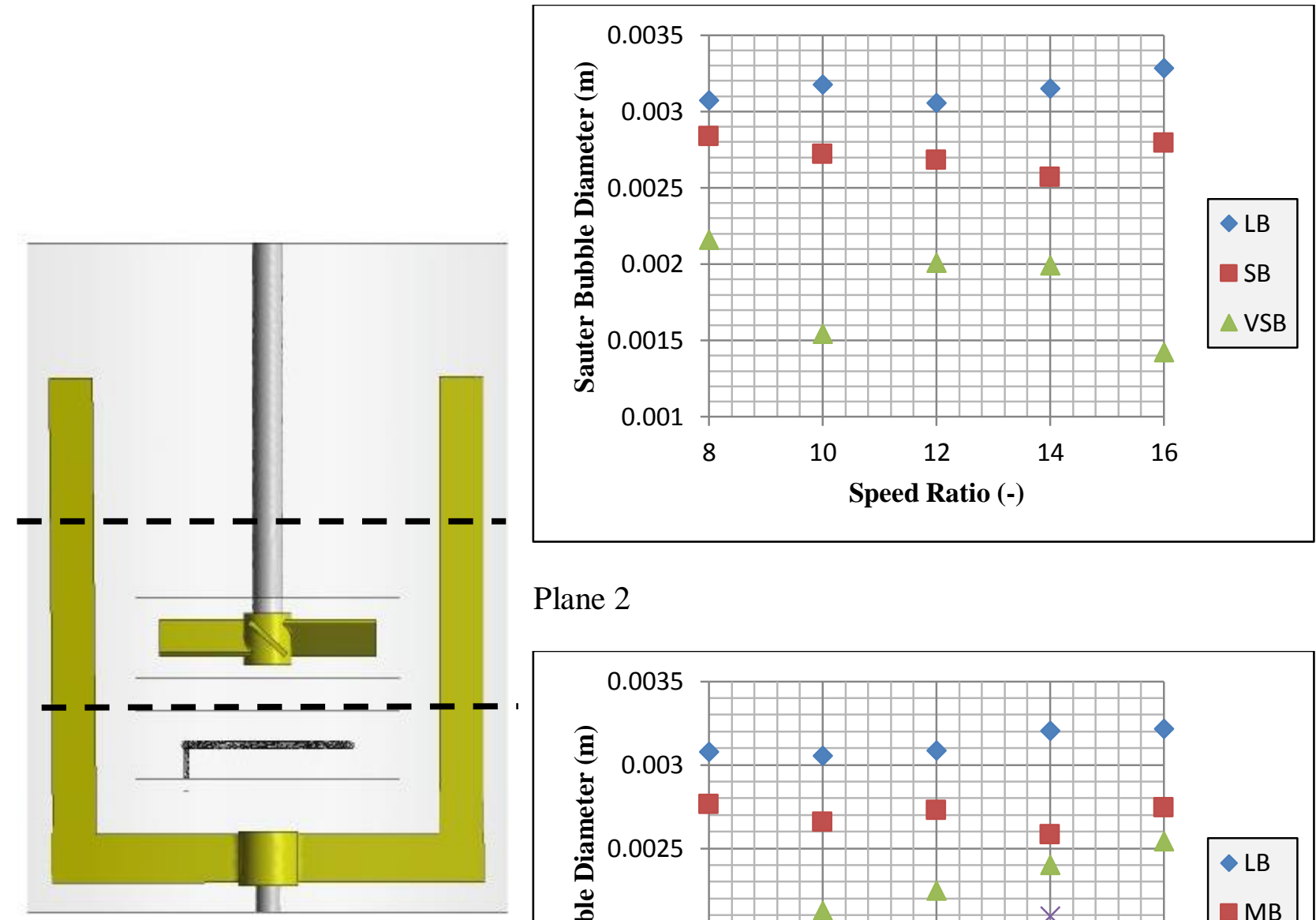

Plane 2

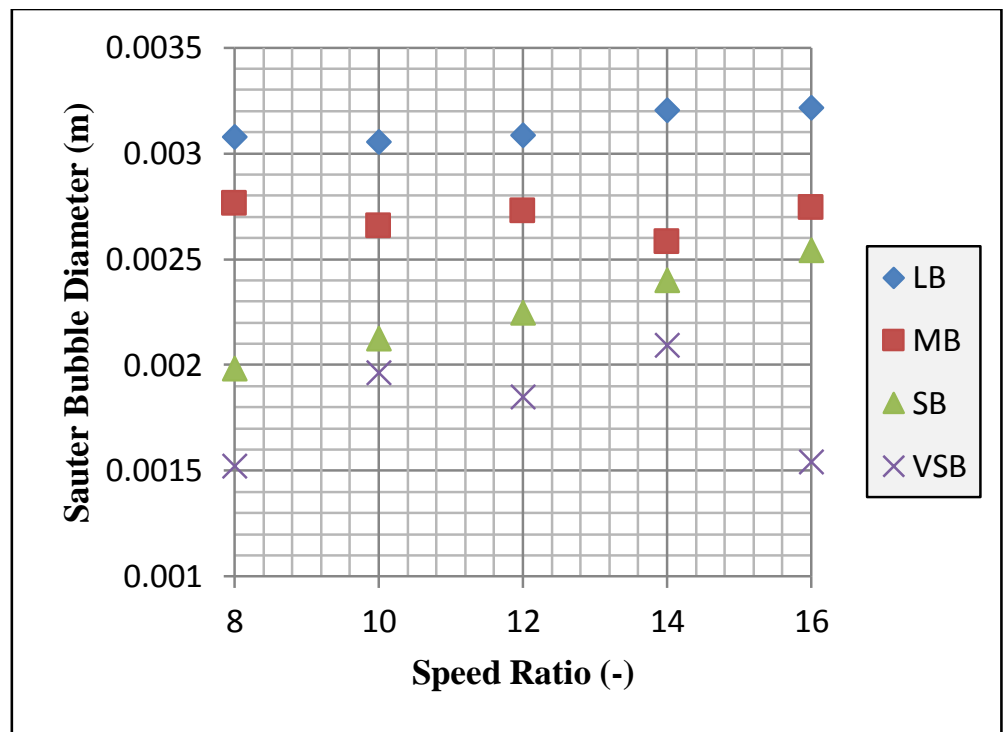

Plane 4

Figure (5.3- 5). Effect of the speed ratio on the Sauter bubble diameters captured at (a) plane 2 and (b) plane 4. 


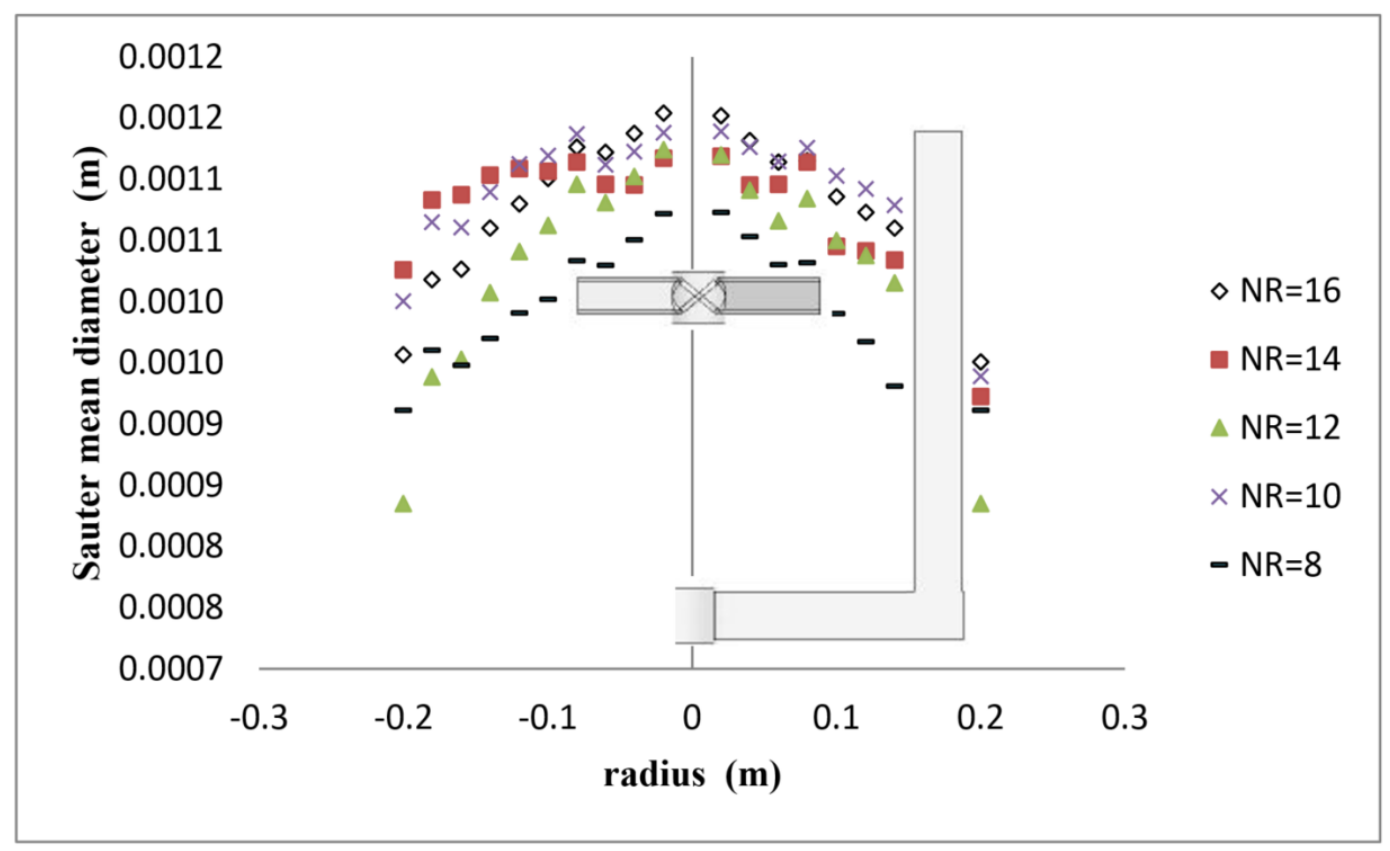

Figure (5.3- 6). Relation between the Sauter bubble diameter and the speed ratio at the central impeller height.

\subsubsection{Conclusion}

It was observed that at the fixed central impeller speed and the gas velocity, the number of bubble size classes, which were identified at two different heights of the aerated coaxial mixing vessel, was not varied by changing the speed ratio. However, the percentage of the overall gas holdup, which was occupied by each class of bubbles, was reduced by increasing the speed ratio at constant gas velocity. Also, the obtained results revealed that the local gas holdups measured in the vicinity of the vessel wall were increased as the speed ratio increased up to the critical value of 10 . Nevertheless, by further increase of the speed ratio, the local gas holdup was affected inversely. The minimum gas holdup was achieved at the speed ratio of 10 at the different gas velocities employed in this study. At all considered speed ratios, higher superficial gas velocities led to an increase in the gas holdup. 


\section{Chapter 5: Results and Discussion}

With respect to the bubble sizes, they were varied by changing the speed ratio and the largest bubbles were observed at the higher speed ratios. It was demonstrated that the smallest bubble sizes generated at the central impeller plane. Besides, increasing the anchor speed increased the bubble breakage and resulted in the smaller bubbles near the vessel wall. The bubble coalescence happened as the sparged bubbles traveled upward and their sizes were varied by both the speed ratio and the gas velocity. 
Chapter 5: Results and Discussion

\subsection{Hydrodynamic characteristics of an aerated coaxial mixing vessel equipped with a pitched blade turbine and an anchor}

\subsubsection{Introduction}

In the past decades, remarkable efforts have been carried out to enhance the performance of the gas-liquid mixing systems. Mass transfer from the gas phase to the liquid phase has been defined as a significant factor for evaluating the performance of the vessels involving chemical reactions or biological processes. One of the most important characteristics affecting the mass transfer between gas and liquid phases is the gas holdup. To be illustrated, in the fungal bioreactors an appropriate aeration is necessitated for continuous oxygen supply since oxygen is an indispensable nutrient consumed by micro-organisms for their growth and metabolism (Sarkar et al., 2016; Liu et al., 2006; Arrua et al., 1990). The performance of the aforementioned bio-processes is notably affected by insufficient oxygen dispersion. Therefore, the desire to eliminate the oxygen-starved stagnant zones has attracted many attentions.

In the aerated mixing vessel, obtaining the detailed information on global and local gas holdups is essential for better understanding of the complex interaction between the gas and liquid phases and also to reflect the mixing efficiency and mass transfer rate. In addition, the knowledge of the fluid flow developed through the gas-liquid mixing vessels is vital for design and scale-up of these systems. The flow phenomena show considerable complexities and different behavior, which are related to the geometrical and operating conditions of the aerated mixers.

In addition to the experimental measurement, the computational fluid dynamics (Wang et al., 2014; Jahoda et al., 2009; Montante et al., 2008) has been extensively employed as a useful tool for gaining full insight of the flow hydrodynamics developed in the mixing vessels. Also, CFD 
Chapter 5: Results and Discussion

technique is more time efficient in comparison with the experimental methods, as a result of the rapid development of the computer capacity.

In the current study, for the first time the CFD approach was used to verify the effect of different operating parameters such as the speed ratio (i.e. the speed of the central impeller over the speed of the anchor), rotation mode, and fluid viscosity on the global and local gas holdup values within the aerated coaxial mixing vessel. Also, an attempt was made to completely investigate the hydrodynamics of two-phase flow developed in such a bioreactor. To validate the CFD model, the numerical outcomes for the gas holdup and total gassed power were compared with the experimental data, see section 4.3.2.

\subsubsection{Results and Discussions}

\subsubsection{Effect of the speed ratio on the gas holdup at different syrup viscosities}

Gas holdup is determined as an indication for evaluating the effectiveness of mass transfer rate between gas and liquid phases being mixed in the mechanically agitated vessels. As mentioned before, the aerated coaxial mixing vessel consisted of two independent impellers (a central impeller and an anchor), which can turn at different speeds. In an aerated coaxial mixer, although the central impeller plays the key role to disperse the gas throughout the tank, the gas holdup can also be altered by the anchor rotation. The developed flow pattern and the internal parts of the vessel can impact the dispersion of the gas phase in the coaxial tank. In this work, the central impeller speed was fixed at $1600 \mathrm{rpm}$, and the anchor speed was varied, resulting in four different speed ratios of $8,10,12$, and 14 . It should be mentioned that in the current study only loading/complete gas dispersion flow pattern was considered. Figures 5.4-1 shows the effect of the speed ratio on the gas dispersion at two viscosity values. It is evident that when the viscosity increased at a constant central impeller speed, the average gas holdup was decreased. In our latest 


\section{Chapter 5: Results and Discussion}

section (5.3), it was found that in an aerated coaxial mixing vessel comprising the corn syrup solution with a viscosity equal to $0.05 \mathrm{~Pa}$.s, the speed ratios higher than 10 led to the higher gas volume fractions within the vessel. From Figure 5.4-1, it can be seen that the overall gas holdup was maximized at the critical speed ratio of 12 . Therefore, the results obtained from our experimental and modelling studies demonstrated that the aerated coaxial mixing vessels were more efficient in term of gas holdup irrespective of employed viscosity at the speed ratios higher than 10. Figure 5.4-2 the contour plots of the gas dispersion inside the coaxial mixing vessel at different viscosities and speed ratios. These results showed that although at the speed ratio of 14 the averaged gas holdup was less than that acquired at the speed ratio of 12 , the joined flow pattern induced by both central impeller and anchor increased the centrifugal forces leading to the enhancement of the gas phase distribution throughout the coaxial mixer. The possible explanation for the higher averaged gas holdup at the speed ratio of 12 can be related to the higher volume of the gas phase appearing beneath and above the central impeller. Also, from these contour plots it is obvious that at the speed ratio of 8 , the gas phase was not observed in the vicinity of the vessel walls. The highest gas holdup was attained adjacent to the central impeller for all considered speed ratios while the amount of gas phase was reduced near the top shaft, especially at the lower speed ratios. In fact, these data demonstrated that when the speed of the pitched blade turbine was fixed, the gas distribution was enhanced with a decrease in the anchor speed, which was corresponded to a higher speed ratio. This may be due to the fact that at the higher anchor speed, the tangential flow pattern generated by the anchor negatively affected the distribution of the gas phase. 
Chapter 5: Results and Discussion

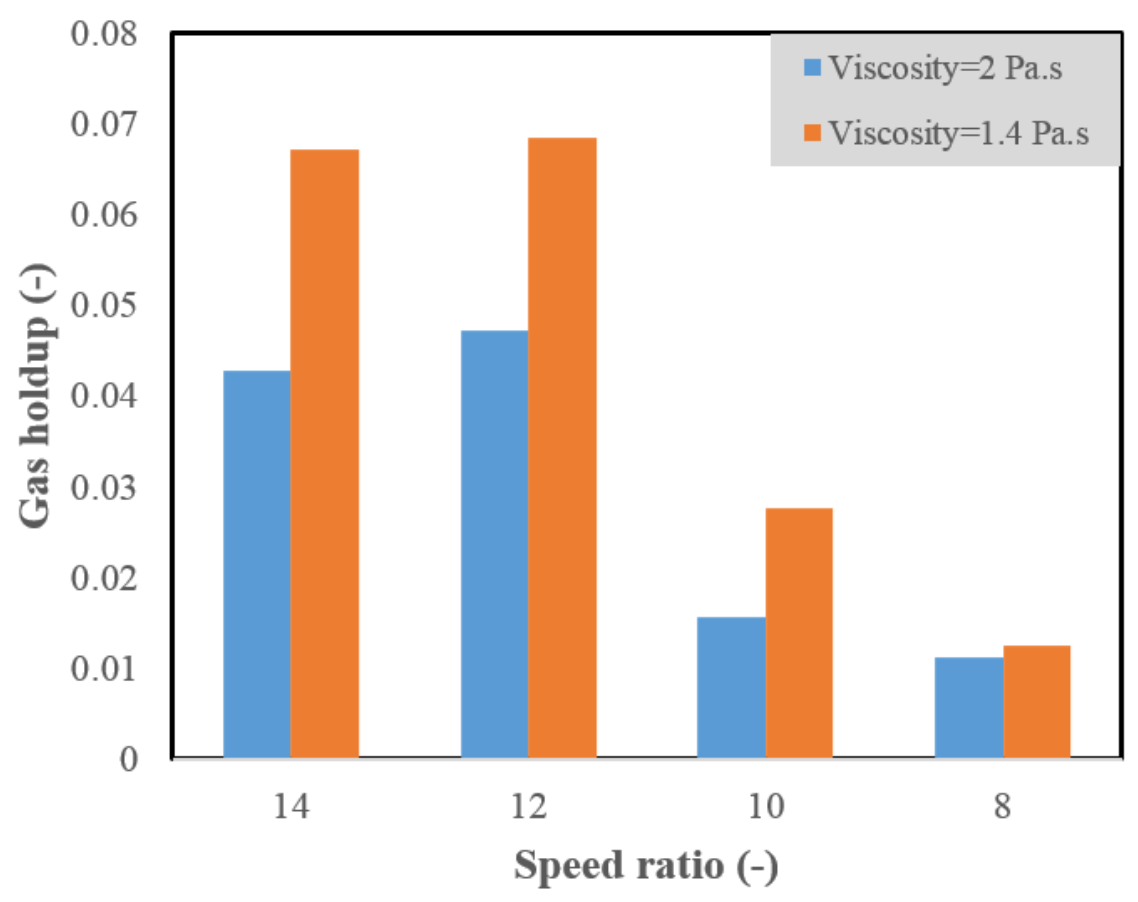

Figure (5.4- 1). Gas holdup values in an aerated coaxial mixer as a function of the speed ratio and fluid viscosity. 


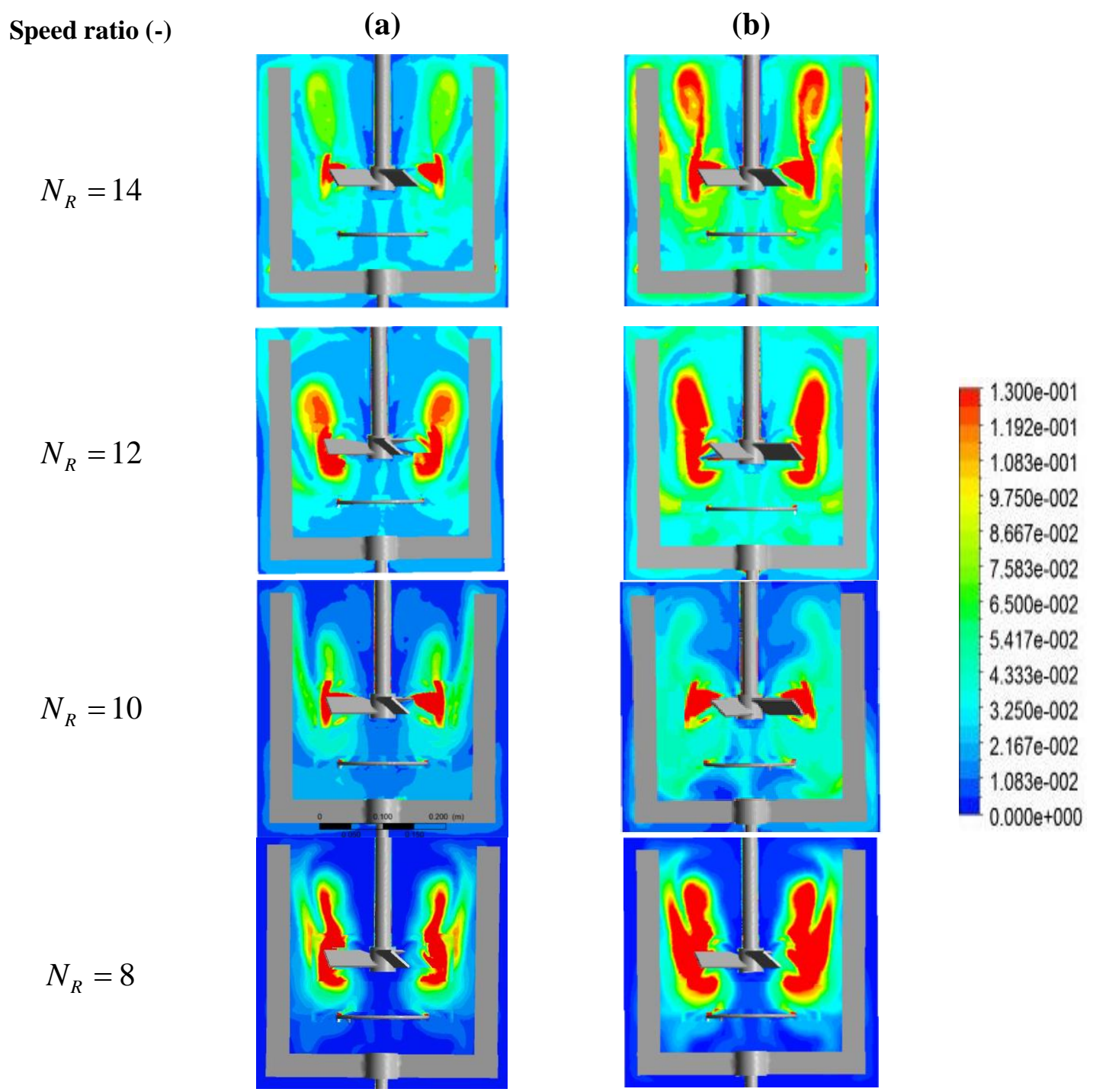

Figure (5.4- 2). Contour plots of gas dispersion throughout the coaxial mixing system at different speed ratios for: (a) $\mu=2$ Pa.s, and (b) $\mu=1.4$ Pa.s

In addition, the time averaged local gas holdups were also assessed at five axial positions $(z=$ 0.09, 0.146, 0.202, 0.258, and $0.314 \mathrm{~m}$ ) as shown in Figure 3.2-1. The aforementioned vertical planes named from top downward. The vertical positions were selected at the same heights of the ERT planes employed in our experimental studies. From Figure 5.4-3 it can be seen that at all selected axial positions, the gas holdup was higher at the speed ratios higher than 10 . This is a desired condition for the fermentation processes, where a sufficient supply of oxygen is 


\section{Chapter 5: Results and Discussion}

necessitated for microorganism. It was observed that beneath the sparger, Plane 5, at the radial locations closer to the middle of the tank, $r / R<0.4$, the gas holdups were higher at the speed ratio of 12 compared to those obtained at the speed ratio of 14 . However, beyond the aforementioned locations the volume fraction of the gas was increased at the speed ratio of 14. The comparison of the gas volume fraction profiles on plane 4 revealed that at $r / R<0.17$ and $r / R>0.6$, very small differences were found between the gas holdups achieved at the speed ratios of 12 and 14 . At the level of the central impeller, Plane 3, the gas holdup profiles attained at the speed ratios of 12 and 14 were almost overlapped, probably because of the gas cavities generated around the central impeller blades. Above the central impeller, Planes 1 and 2, near the walls and the top shaft, the difference between the gas volume fractions obtained at the speed ratios of 12 and 14 was not significant. Nonetheless, the main difference between these speed ratios, 12 and 14, was related to the formation of the bigger vortex by the anchor blades at the speed ratio of 12 (see Figure 5.4-4). As it can be seen from Figure 5.4-4, in the upper part of the vessel and at the low pressure regions behind the anchor blades vortices were appeared, and the size and strength of these vortices were affected by the speed ratio. The vortex was smaller at the lower speed ratio (i.e. at the higher anchor speed). Thus, the speed ratio of the coaxial mixer must be more than 10 in order to enhance the gas distribution inside the aerated coaxial mixing vessel. 
Chapter 5: Results and Discussion
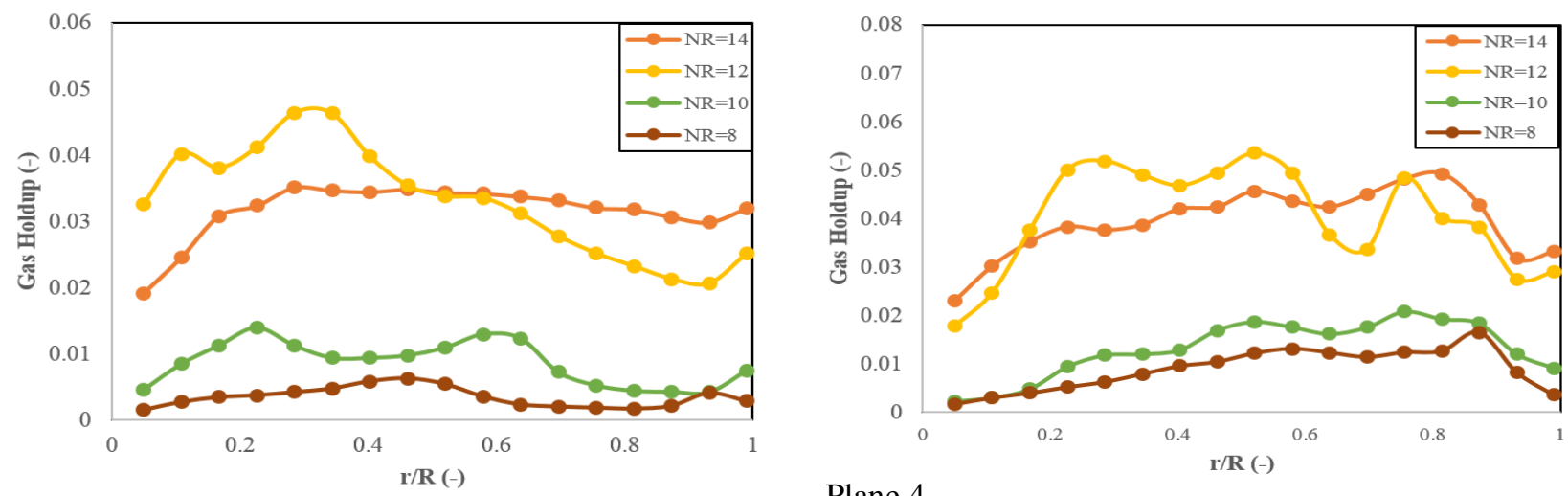

Plane 5

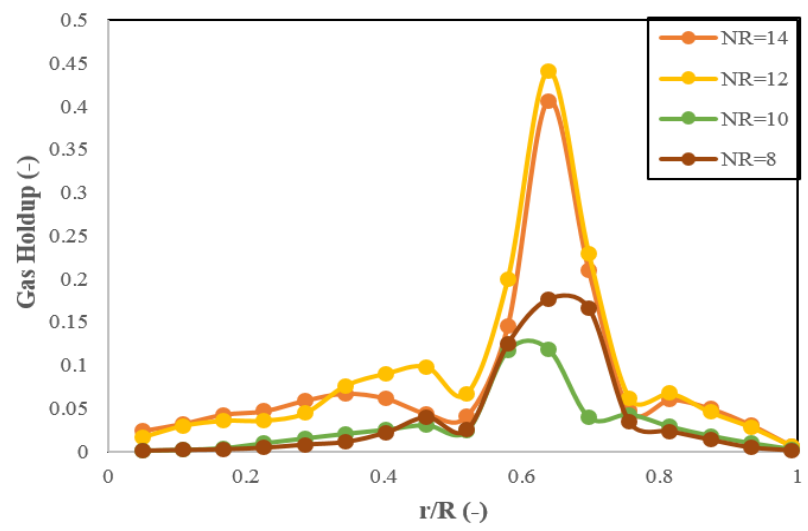

Plane 4

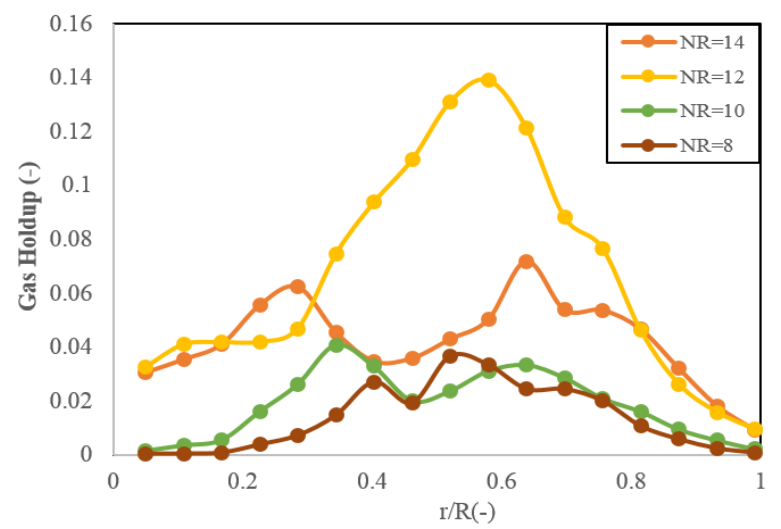

Plane 2

Plane 3

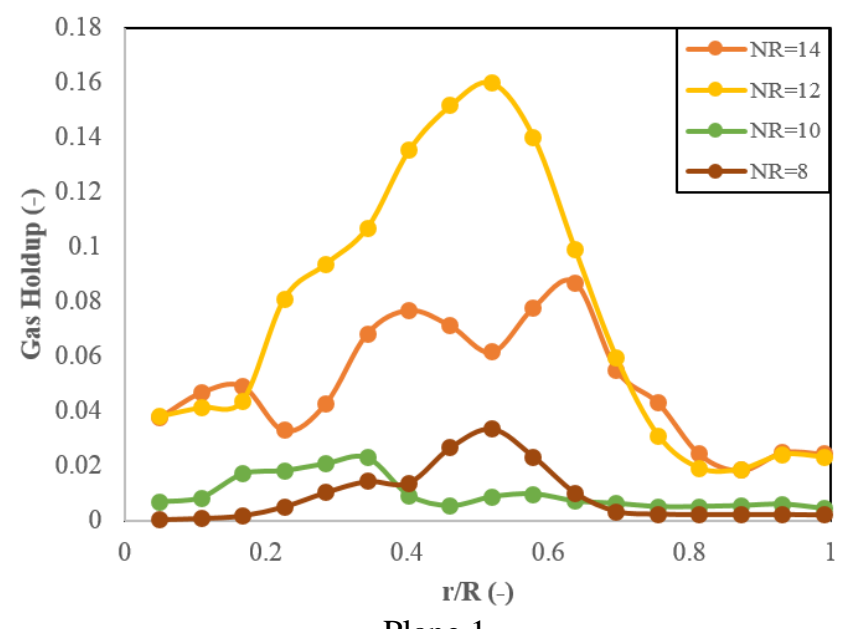

Plane 1

Figure (5.4- 3). Local gas holdup values at different speed ratios ( $\mu=2$ Pa.s).

The contours of the gas holdup distribution in the aerated coaxial mixing vessel at the aforementioned vertical planes have been presented in Figure 5.4-4. As it can be seen, at the speed ratio of 12 , the sizes of the developed vortices at the central impeller level and above of that (Planes 
3,2 , and 1) were bigger than those formed at other speed ratios. Furthermore, Figure 5.4-4 supports this fact that the variations of speed ratio did not affect the shape of the gas cavities formed on the anchor blades at the different heights of the aerated coaxial mixer. Nonetheless, the size of the gas cavities was significantly dependent on the employed speed ratio. In addition, the size of the gas cavities generated beneath the central impeller blades were affected by the speed ratio, see plane

3.

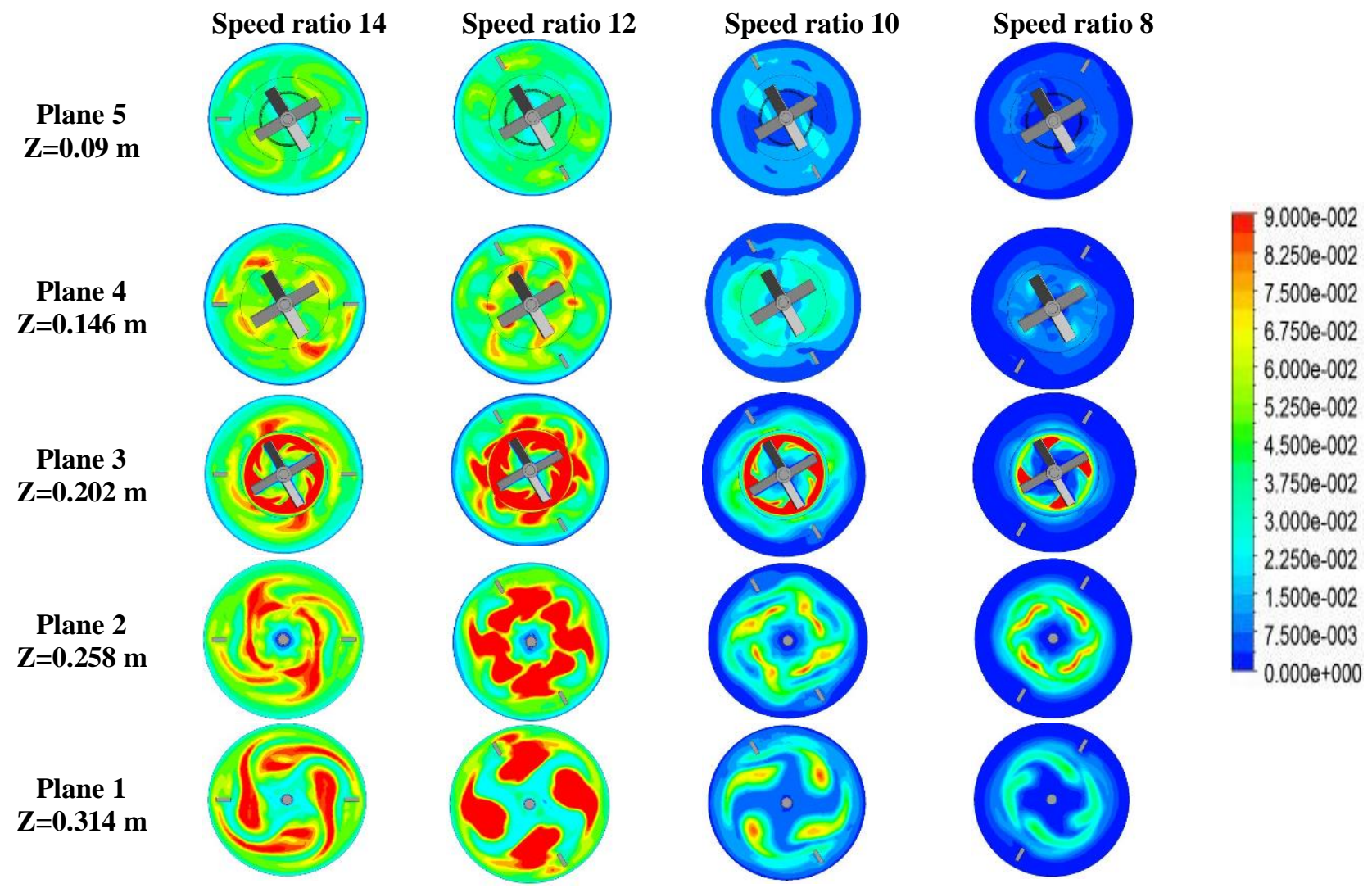

Figure (5.4- 4). Gas holdup distributions at different heights $(z=0.09,0.146,0.202,0.258$, and, $0.314 \mathrm{~m}$ ) of the aerated coaxial mixer and different speed ratios. 
Chapter 5: Results and Discussion

\subsubsection{Effect of rotation mode on the gas holdup}

The effect of the speed ratio on the gas dispersion of the counter-rotating coaxial mixer was investigated at the same operating conditions as previously conducted for the co-rotating mode. The gas holdup contours achieved in the co-rotating and counter-rotating modes have been presented in Figure 5.4-5. As mentioned earlier, the speed ratios greater than 10 provided a higher gas distribution throughout the vessel in the co-rotating mode. However, as it can be seen in Figure 5.4-5, the gas holdup was higher at the speed ratios lower than 10 in the counter-rotating mode. In order to analyze this behavior, the turbulence kinetic energy within the coaxial mixing vessel in the co- and counter-rotating modes were assessed qualitatively and quantitatively (see Figures 5.46 and 5.4-7). The theory of mixing operations in the aerated mechanically agitated vessels in turbulent regime is based on the Kolmogorov theory of turbulence, at which the main parameter is defined as the local dissipation rate of turbulent kinetic energy. In the turbulent gas-liquid mixing systems, the local gas holdup values, are related to the turbulent kinetic energy and dissipation rates (Vlachakis, 2006). In the aerated coaxial agitated tanks, the aforementioned turbulent parameters can be affected by the rotation mode and the speed ratio. The turbulence kinetic energy in the co- and counter-rotating coaxial mixers have been compared at five different vertical positions in Figure 5.4-7. When the rotation of the pitched blade impeller and the anchor was in the opposite directions, the turbulent kinetic energy was lower in the most radial positions, especially near the anchor blades, than those obtained for the aerated coaxial mixer in the corotating mode, at the same speed ratio. However, in the central impeller zone, the difference between the turbulent kinetic energies of both modes was inconsiderable. In all considered cases, as $r / R$ increased the generated flow by the central impeller tended to lose its momentum, and at these regions the speed and rotation mode of the anchor affected the developed kinetic energy. 


\section{Chapter 5: Results and Discussion}

Therefore, it can be concluded that in the vicinity of the central impeller the dependency of the turbulent characteristics on the anchor speed and its rotational direction was negligible. The minimum values of the turbulent kinetic energy were attained beneath the sparger and the bottom corners of the aerated coaxial mixer. The aforementioned phenomenon was highlighted at the speed ratios higher than 10 when the central impeller and the anchor were rotating at the opposite directions. The higher the kinetic energy, the more the large eddies are broken down into the small ones resulting in higher gas volume fractions. Therefore, due to the presence of the higher kinetic energy in the co-rotating mode compared to that in the counter-rotating mode at $N_{R}>10$, the corotating aerated coaxial mixer leading to a higher gas holdup.

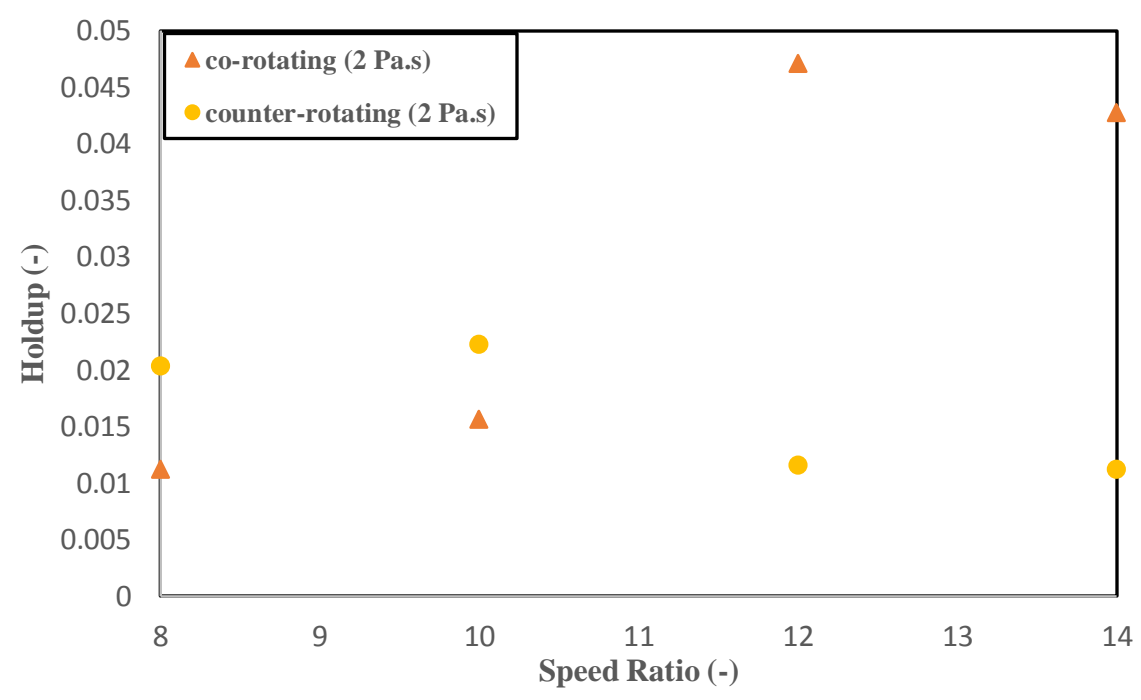

Figure (5.4- 5). Gas holdup at the different speed ratios in the co-rotating and counter-rotating modes. 
(a)

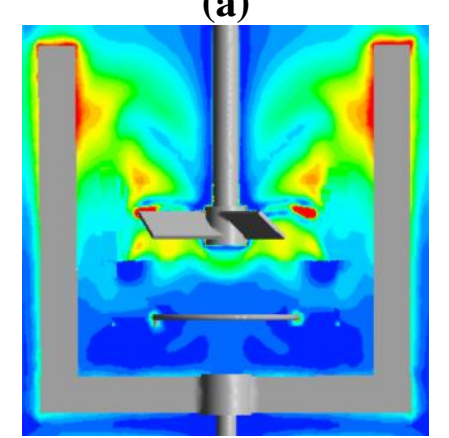

(e)

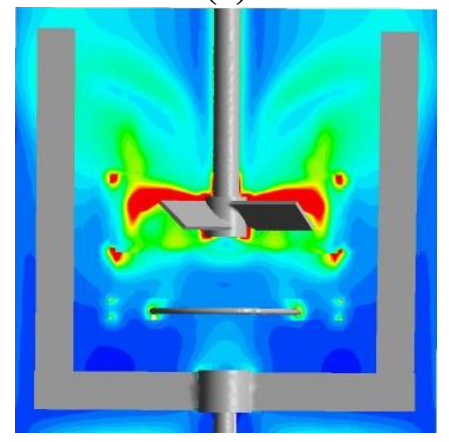

(b)

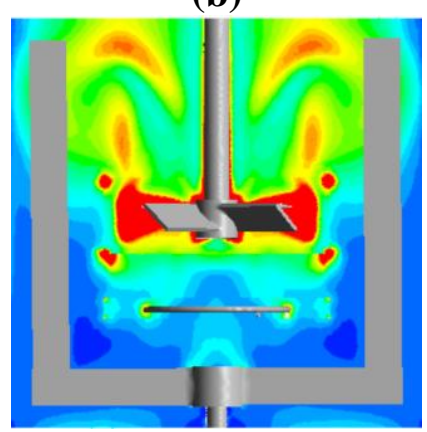

(f)

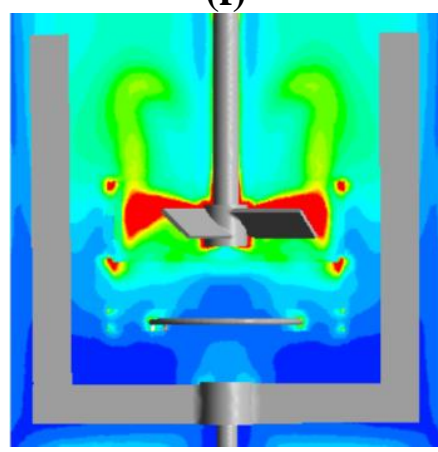

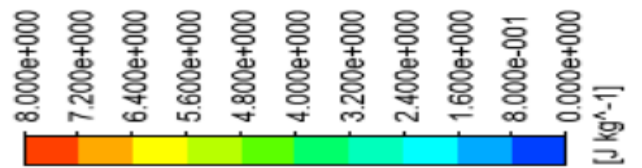

(c)

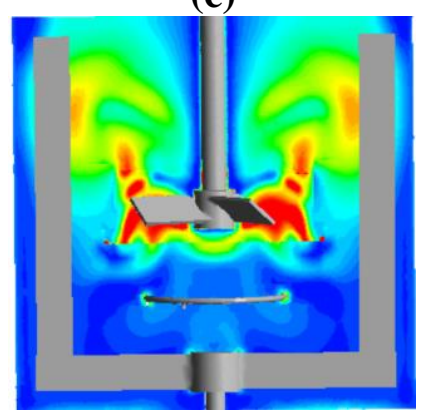

(g)

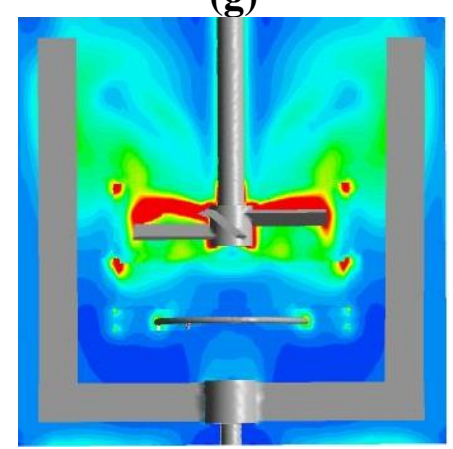

(d)

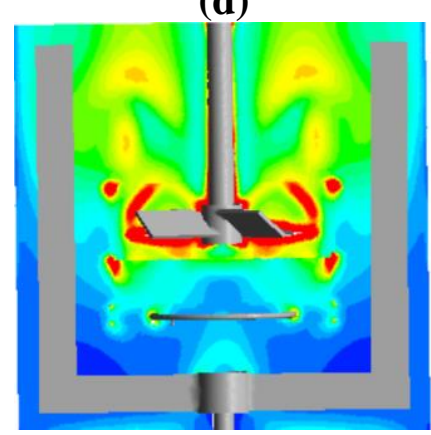

(h)

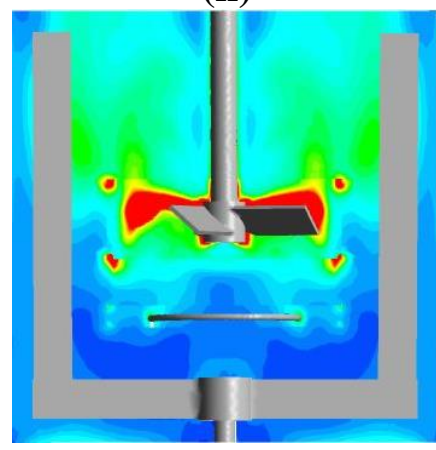

Figure (5.4- 6). Contour plots of turbulent kinetic energy at: (a) counter-rotating $N_{R}=14$, (b) co-rotating $N_{R}=14$, (c) counter-rotating $N_{R}=12$, (d) co-rotating $N_{R}=12$, (e) counter-rotating $N_{R}=10$, (f) co-rotating $N_{R}=10,(\mathrm{~g})$ counter-rotating $N_{R}=8$, (h) corotating $N_{R}=8$. 


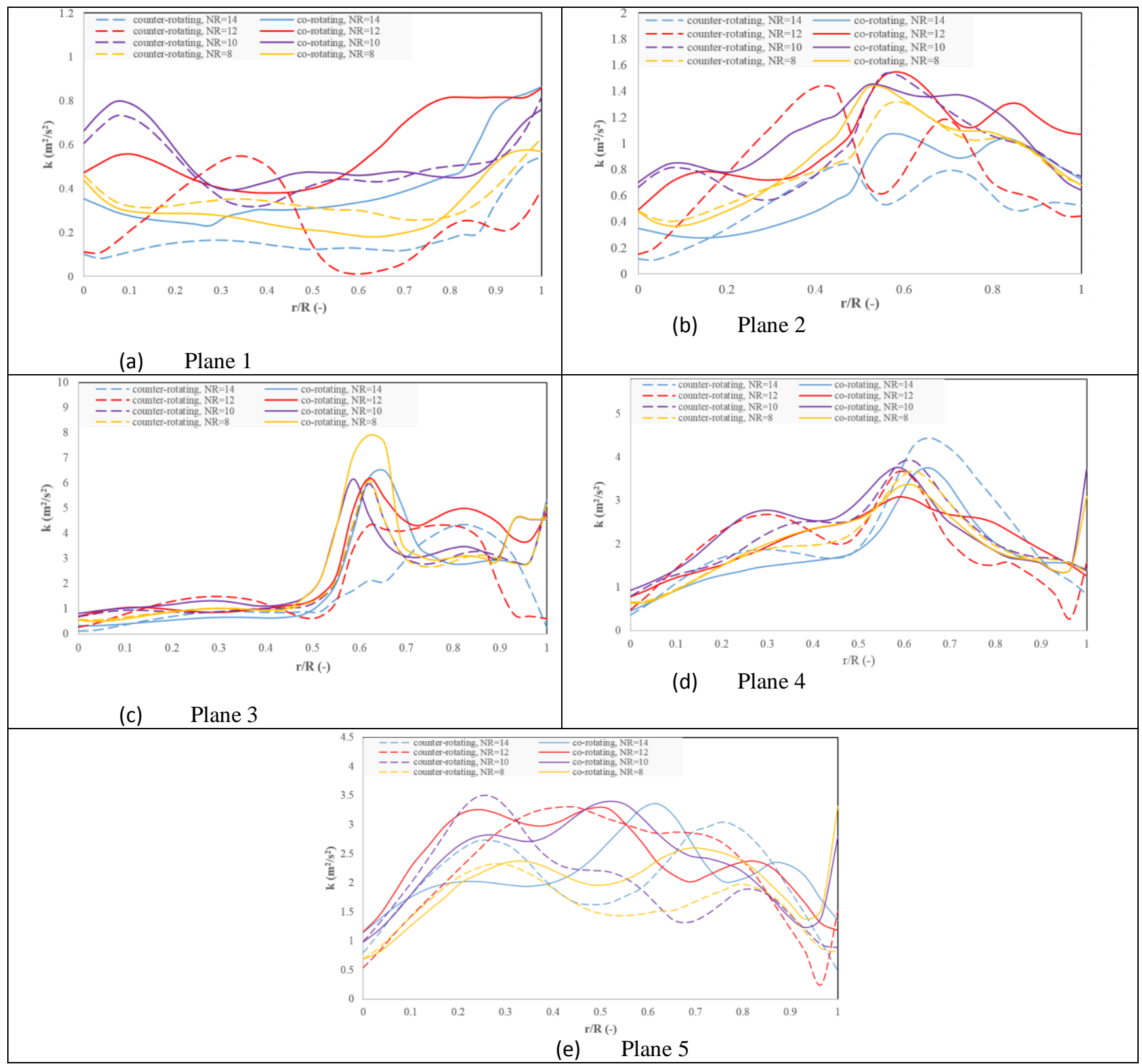

Figure (5.4- 7). Local turbulent kinetic energy data at different heights $(Z=0.09,0.146,0.202$, 0.258 , and, $0.314 \mathrm{~m}$ ) for the co- and counter-rotating aerated coaxial mixer. 
Chapter 5: Results and Discussion

\subsubsection{Flow field}

Computational fluid dynamics enables the flow visualization in the agitated tanks, and can be served for better understanding of the phenomena occurred during the experiments. Figure 5.4-8 shows the velocity vectors generated by the co-rotating coaxial mixer. The central impeller was rotating at $1600 \mathrm{rpm}$ and the anchor was rotating at the speed ratios of $14,12,10$, and 8 . The gas flow rate was $0.0283 \mathrm{~m}^{3} / \mathrm{min}$. It should be noted that the presented velocity vectors are two dimensional, and the colors of the vectors indicate the velocity magnitudes. According to Figure 5.4-8, the flow pattern developed by the central impeller in all speed ratios was the axial flow, which mostly occupied the upper part of the mixing vessel, above Plane 4. As it can be seen, in addition to the axial flow generated by the central impeller, some much weaker circulation loops were developed at different parts of the mixing vessel especially near the surface and bottom of the vessel. The size and the number of the smaller circulation loops were dependent on the speed ratio. For example, the velocity profile generated at the speed ratio of 8 comprised several secondary circulation loops, two near the liquid surface and two at the vicinity of the bottom of the tank. The complex interaction between the flows induced by the central impeller, anchor, and gas injection was the main reason for development of the aforementioned secondary circulation loops throughout the coaxial mixer. 


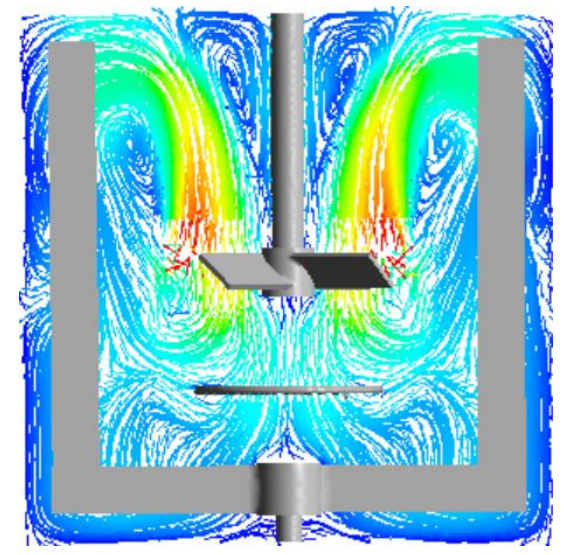

(a)

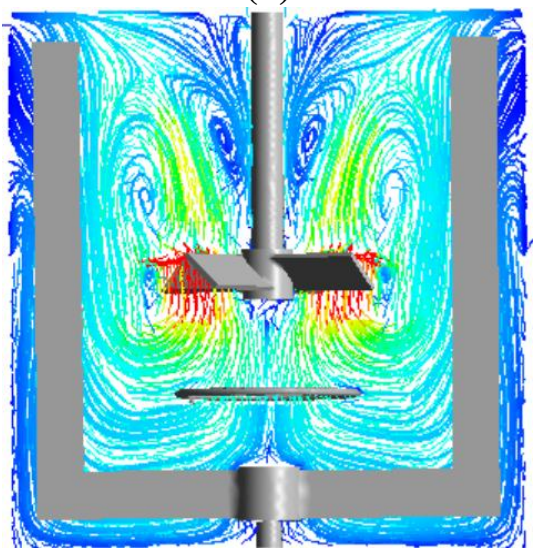

(c)

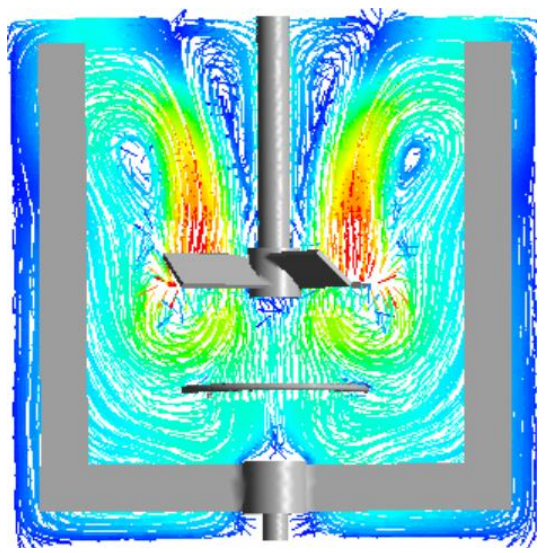

(b)

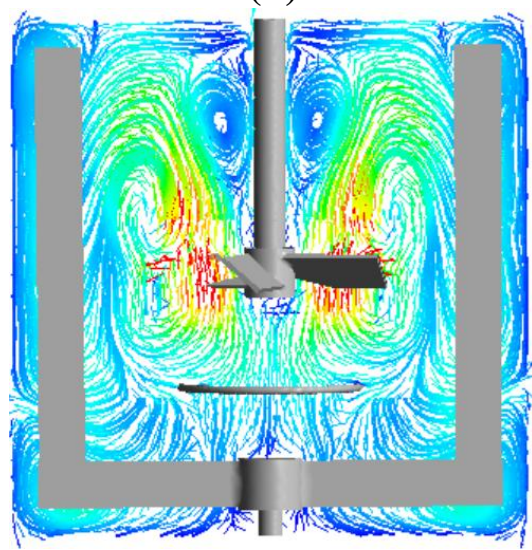

(d)

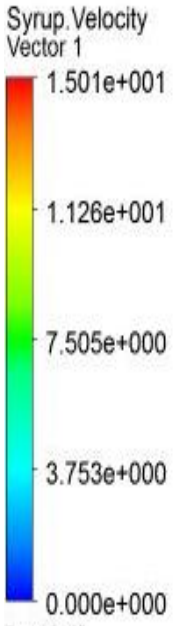

$\left[\mathrm{ms}^{\wedge}-1\right]$

Figure (5.4- 8). Velocity vectors generated by the aerated coaxial mixer in the co-rotating

mode: (a) $N_{R}=14$, (b) $N_{R}=12$, (c) $N_{R}=10$, (d) $N_{R}=8$

\subsubsection{Conclusion}

In the current study, a CFD model was developed to assess the hydrodynamics of the aerated coaxial mixing vessel equipped with an upward pumping pitched blade turbine (central impeller) and a wall scraping anchor. Experimental data of the gas holdup and gassed power uptake were corroborated by the CFD results. In all carried out simulations, the pithed blade impeller speed was set at $1600 \mathrm{rpm}$ and the anchor speed was changed to achieve the speed ratios of $14,12,10$, and 8. Since oxygen mass transfer which is function of the gas volume fraction is the main concern in a number of gas-liquid mixers, the effects of the speed ratio, rotation mode, and fluid viscosity 


\section{Chapter 5: Results and Discussion}

on the dispersed gas holdup throughout the aerated coaxial mixing vessel were investigated. In addition, in the turbulent gas-liquid agitated vessels, the bubble size is dependent on the turbulent characteristics such as the turbulent kinetic energy. Therefore, the effects of the speed ratio and rotation mode on the aforementioned turbulent parameter were assessed as well. It was revealed that at a fixed central impeller speed, increasing the fluid viscosity affected the gas holdup negatively. Additionally, from both experimental and simulation studies, it was found that in the aerated coaxial mixing vessels, the speed ratios higher than 10 led to the higher gas volume fractions regardless of the fluid viscosity. By analyzing the time averaged local gas holdups at the different radial position along the height of the coaxial mixer, it was found that near the tank walls the gas holdups were increased at the speed ratio of 14 . However, the gas holdup in the vicinity of the $z$ axis of the vessel, especially beneath the sparger, was higher at the speed ratio of 12 . Interestingly, the shape of the vortex formed by the anchor blades was not affected by the anchor speed; however, the size of the vortex was strongly dependent on the anchor speed. The co-rotating coaxial mixer outweighed the counter-rotating one in term of gas dispersion. Besides, the counterrotating coaxial mixer was more efficient at the speed ratios lower than 10 . The turbulent kinetic energy attained in the counter-rotating mode was lower than those for the co-rotating coaxial mixer in the most regions within the mixing tank, especially near the vessel walls. The size and the number of circulation loops developed within the coaxial mixer were affected by the speed ratio. 
Chapter 5: Results and Discussion

\subsection{Modeling of bubble size distribution in a bioreactor equipped with a coaxial mixer using population balance model coupled with CFD}

\subsubsection{Introduction}

The main challenge in the aerobic fermentation technologies is supplying sufficient oxygen (air) into the fluid which is essential for the growth of the microbial cells. This difficulty is raised in high cell density bioreactors, at which mass transfer rate between the gas and liquid phases is a limiting step affecting cell growth and productivity. Therefore, adequate aeration for persistent oxygen supply is necessitated to prevent cellular damage within the bioreactors. The mass transfer rates depend on the local values of gas volume fraction and bubble size distribution within the aerated mixing vessels. The lack of knowledge regarding the bubble breakup and coalescence leads to sub-optimally designed fermenters (Prince and Blanch, 1990).

Computational fluid dynamics has become a proper tool to simulate developed hydrodynamics throughout the mixing tanks. In a number of published works, a constant bubble size was assumed in modelling of aerated mixers due to the simplicity, lack of experimental data, and lesser computational time. This idea was the same as what we did in section 5.4. However, in the real system, bubble breakup and coalescence happen due to the bubble-bubble and bubble-liquid interactions, resulting in bubble size distributions in the mixing vessels. To tackle this problem a population balance model coupled with CFD is mainly utilized.

In the aerated coaxial mixing vessels due to the rotation of two independently driven impellers (central impeller and anchor) the generated bubble sizes can be affected by more factors than those developed in the traditional mixing vessels equipped with a single or multiple impellers placed on the same shaft. For example, in the aerated coaxial mixers, the speed ratio (central impeller speed over anchor speed) and the rotation modes (co-rotating or counter-rotating) of the impellers can 
Chapter 5: Results and Discussion

affect the dispersed bubbles within the tank. In the previous sections, it was demonstrated that the co-rotating mode outweighed the counter-rotating mode in terms of mixing time, power consumption, gas holdup, and turbulent dissipation rate. Therefore, in this work only the effect of the speed ratio on the bubble size distributions throughout the aerated coaxial mixer was considered.

The aim of this study was to model the effect of the speed ratio on the bubble size distribution within the aerated coaxial mixer composed of an upward pumping pitched blade turbine and an anchor. In order to fulfill this objective, local bubble size distributions were simulated using PBM in conjunction with CFD by varying the speed ratio $\left(\mathrm{N}_{\mathrm{R}}=8,10,12,14\right)$. The simulation outcomes were validated by experimental results that were obtained by ERT at the same operating conditions.

\subsubsection{Results and discussions}

\subsubsection{Bubble size distribution}

As mentioned in section 4.1.3, multiple size group (MUSIG) model with bubble breakup and coalescence kernels was implemented to characterize the bubble size distribution within the aerated coaxial mixing vessel. In this approach, the bubbles size range can be displayed by a finite series of discrete classes or bins. In the current study, fifteen bubble bins ranging from 0.5 to 12 $\mathrm{mm}$ were considered as per the data obtained from the tomography measurements. Since the bubble sizes cannot be measured directly by ERT, the correlation proposed by Lagisetty et al. (1986) was used to estimate the bubble sizes:

$$
\frac{d_{b}}{D}=0.125\left(1+4 \phi_{g}\right)^{1.2}\left(\frac{\rho_{l} N^{2} D^{3}}{\sigma}\right)^{-0.6}
$$


Chapter 5: Results and Discussion

here $\phi_{g}, N, D$, and $\sigma$ are gas holdup, impeller speed, impeller diameter, and surface tension, respectively. The gas holdup values were obtained by ERT measurements.

Since the coaxial mixer was composed of two independent impellers, which can rotate at different speeds and directions, an equivalent rotational speed was needed. As explained in details in section 5.2.2.2, Bao's equivalent rotational speed $\left(N_{c}-\frac{N_{a}}{N_{R}}\right)$ was adopted in this study and was substituted in the Eq. (5.5-1). Accordingly, the maximum and minimum bubble sizes were 12.7 mm $0.583 \mathrm{~mm}$, respectively. In all conducted simulations, the central impeller speed and the gas flow rate were $200 \mathrm{rpm}$ and $0.0283 \mathrm{~m}^{3} / \mathrm{min}$, respectively. The anchor speed was varied to achieve the speed ratios of $8,10,12$, and 14 . The detailed of the adopted breakup and coalescence kernels were discussed in sections 4.1.3.1 and 4.1.3.2, respectively.

To define the boundary conditions, the upper surface of the ring sparger from which the air was introduced into the vessel was defined as the velocity inlet, where the gas volume fraction was equal to unity and the velocity of the sparged gas was calculated based on the actual gas flow rate. Another important parameter was the bubble diameter leaving the sperger. Different correlations have been reported in the literature for the estimation of the size of the bubbles produced at the sparger as summarized in Table 5.5-1. 
Chapter 5: Results and Discussion

Table (5.5- 1). Different correlations for the bubble size at the sparger.

\begin{tabular}{|c|c|c|}
\hline Reference & Correlation & Conditions \\
\hline Miller (1974) & $d_{b-s p \arg e r}=\left(\frac{6 \sigma d_{s p \arg e r}}{g\left(\rho_{l}-\rho_{g}\right)}\right)^{1 / 3}$ & For low gas flow rates \\
\hline Kumar et al. (1976) & $d_{b-s p \arg e r}=1.56 \operatorname{Re}^{0.058}\left(\frac{\sigma d_{s p \arg e r}^{2}}{g\left(\rho_{l}-\rho_{g}\right)}\right)^{0.25}$ & $1<\operatorname{Re}<10$ \\
\hline Kumar et al. (1976) & $d_{b-s p \arg e r}=0.32 \operatorname{Re}^{0.425}\left(\frac{\sigma d_{s p \arg e r}^{2}}{g\left(\rho_{l}-\rho_{g}\right)}\right)^{0.25}$ & $10<\operatorname{Re}<2100$ \\
\hline Kumar et al. (1976) & $d_{b-s p \arg e r}=100 \mathrm{Re}^{-0.4}\left(\frac{\sigma d_{s p \arg e r}^{2}}{g\left(\rho_{l}-\rho_{g}\right)}\right)^{0.25}$ & $4000<\operatorname{Re}<70000$ \\
\hline $\begin{array}{l}\text { Moo-Young and Blanch } \\
\text { (1981) }\end{array}$ & $d_{b-s p \arg e r}=0.19 \mathrm{Re}^{0.32} d_{s p \arg e r}^{0.48}$ & $\operatorname{Re}<2000$ \\
\hline
\end{tabular}

From Table 5.5-1, it was obvious that in order to calculate the bubble size at the surface of the sparger, Reynolds number was needed. Based on the explanations provided in section 5.2.2.2, the following $R e$ equation was used to predict the bubble sizes introduced from the sparger into the tank:

$$
\operatorname{Re}=\frac{\rho\left(N_{c} \pm N_{a} / N_{R}\right) D_{c}^{2}}{\mu}
$$

Therefore, for the aerated coaxial mixer operated in turbulent regime, the size of the bubbles formed at the ring sparger can be calculated by:

$$
d_{b-s p \text { arg } e r}=100\left(\frac{\rho\left(N_{c} \pm N_{a} / N_{R}\right) D_{c}^{2}}{\mu}\right)^{-0.4}\left(\frac{\sigma d_{s p \text { arg } e r}^{2}}{g\left(\rho_{l}-\rho_{g}\right)}\right)^{0.25}
$$


Chapter 5: Results and Discussion

The obtained result was $d_{\text {bubble }}=3.22 \mathrm{~mm}$, hence the volume fraction of the $6^{\text {th }}$ bubble bin with diameter of $3.17 \mathrm{~mm}$ was set to one in the inlet boundary condition.

After the bubbles left the sparger, because of the developed hydrodynamics throughout the tank the breakup and coalescence happened. As a result, bubbles with different sizes distributed within the aerated coaxial mixer. In Figure 5.5-1, the simulated Sauter mean bubble diameters were compared with the experimental ones which were obtained by the following correlation:

$$
d_{s}=4.15\left(\frac{\sigma^{0.6}}{\left(P / V_{l}\right)^{0.4} \rho_{l}^{0.2}}\right) \phi_{g}^{0.2}\left(\frac{\mu_{g}}{\mu_{l}}\right)^{0.25}+0.0009
$$

where $\sigma, \mu, \rho, P / V$, and $\phi_{g}$ are surface tension, viscosity of the liquid (l) and gas phase $(\mathrm{g})$, the liquid's density, total aerated power per liquid volume, and gas holdup, respectively. The gas holdup data were obtained by ERT and substituted in Eq. (5.5-4). The comparison was conducted at one operating condition $\left(N_{c}=200 \mathrm{rpm}, N_{a}=16.7 \mathrm{rpm}\right.$, and $Q_{g}=0.0283 \mathrm{~m}^{3} / \mathrm{min}$ ) and at various locations within the vessel, as depicted in Figure 5.5-1. As it can be seen, the comparison of the predicted and measured values was satisfactory in most parts of the aerated coaxial mixer. However, less agreement was found for the values reported near the vessel wall. This is most likely caused by the errors associated with the obtained local gas holdups values through ERT measurements. All these errors were related to the electrode positions, which were placed on the vessel walls near to the anchor blades. Therefore, rotation of the anchor blades can affect the electrical conductivity, resulting in a high level of noise in the vicinity of the vessel walls. Overall, the adopted modeling approach enabled us to properly characterize the bubble size distribution within the aerated coaxial mixer. 


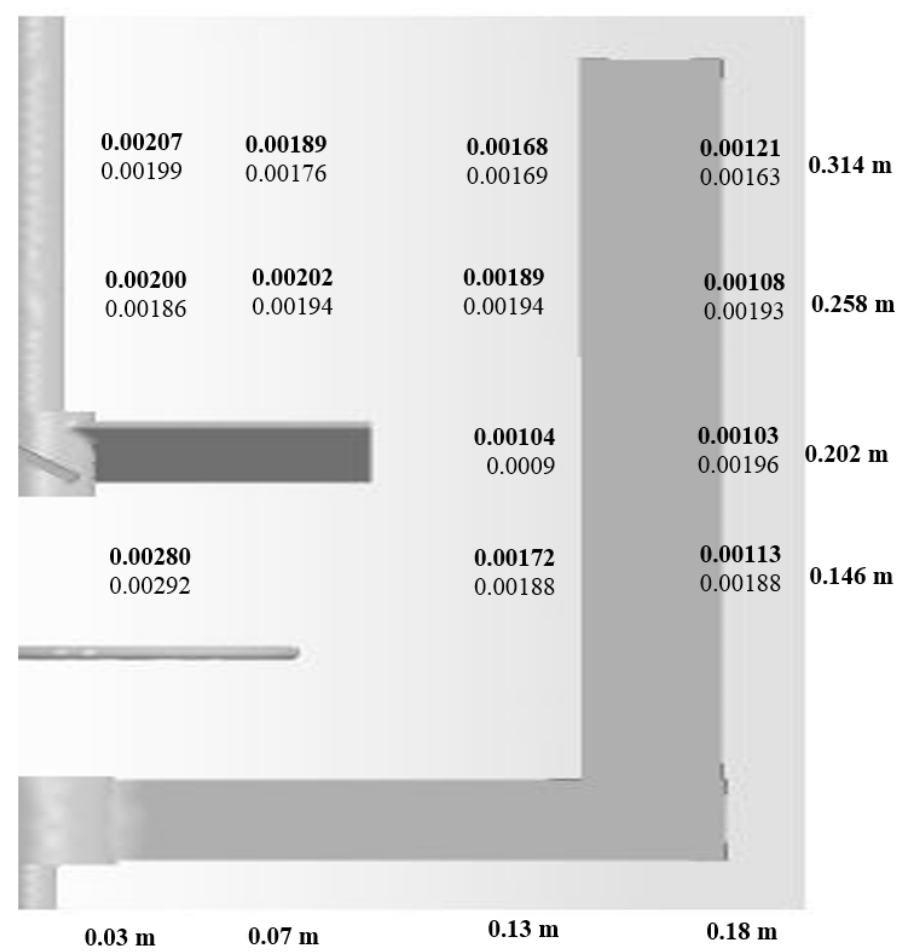

Figure (5.5- 1). Sauter mean bubble diameters estimated through the simulation (bold) and those obtained from the experiment in different points inside of the vessel.

\subsubsection{Effect of speed ratio on the bubble size distributions}

As explained before, the aerated coaxial mixer was furnished with two impellers; one supported with the top shaft and the other attached to the bottom shaft. These two impellers can rotate at different speeds. The ratio of the central impeller speed to the anchor speed $\left(N_{R}\right)$ is an important factor when the performance of the coaxial mixer is investigated in terms of the bubble size distribution. As noted before, the speed ratios of 8, 10, 12, and 14 were considered. These speed ratios were selected based on the works conducted in the previous sections.

In order to investigate the effect of the speed ratio on the bubble volume fractions, the bubbles with different sizes were categorized in three different groups: small, medium, and large bubbles, as depicted in Figure 5.5-2a to 5.5-2c. It can be seen that the speed ratio had an erratic effect on 
the volume fraction of the small and the medium bubble size groups. For instance, the volume fraction of the bubbles with a diameter of $1 \mathrm{~mm}$ was enhanced with an increase in the speed ratio. However, the volume fraction of the bubbles with a diameter of $1.58 \mathrm{~mm}$ decreased when the speed ratio was increased. By increasing the speed ratio, the volume fractions of large bubbles were reduced as can be seen in Figure 5.5-2.c. This resulted in maximizing the interfacial area.

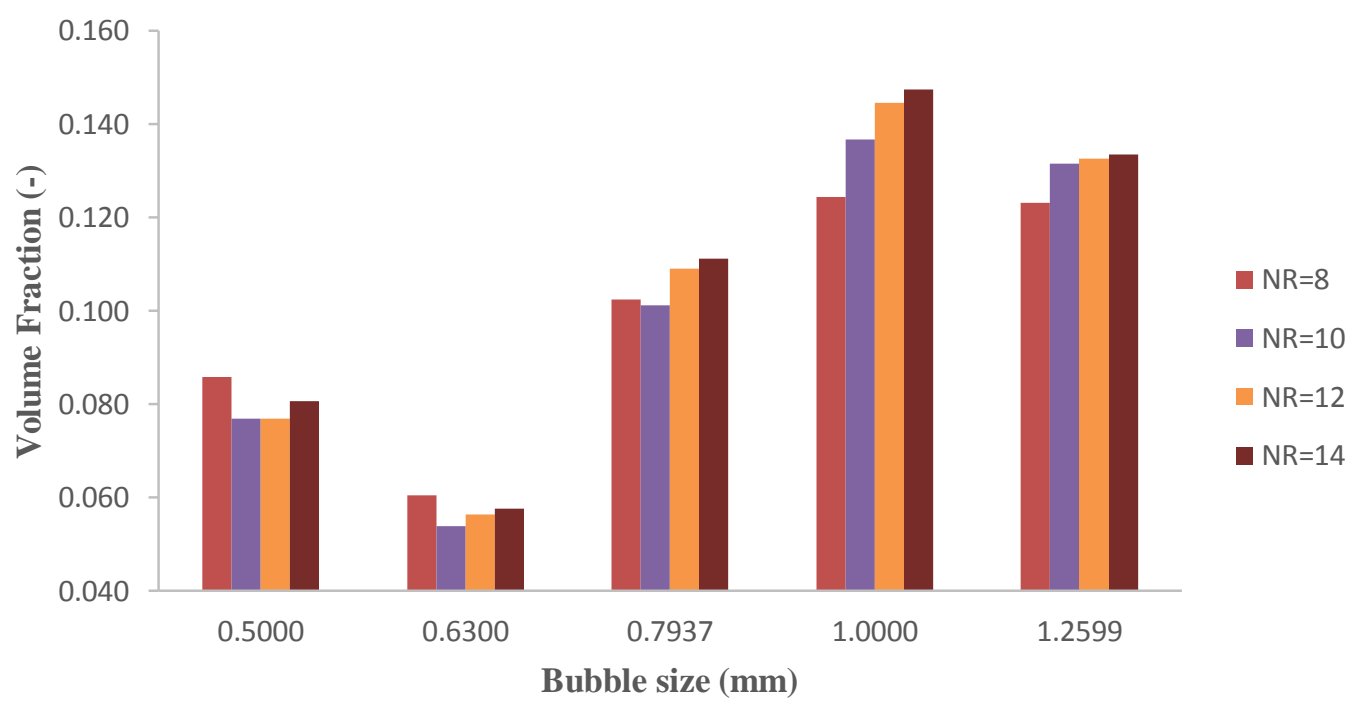

(a) Small size bubble group 
Chapter 5: Results and Discussion

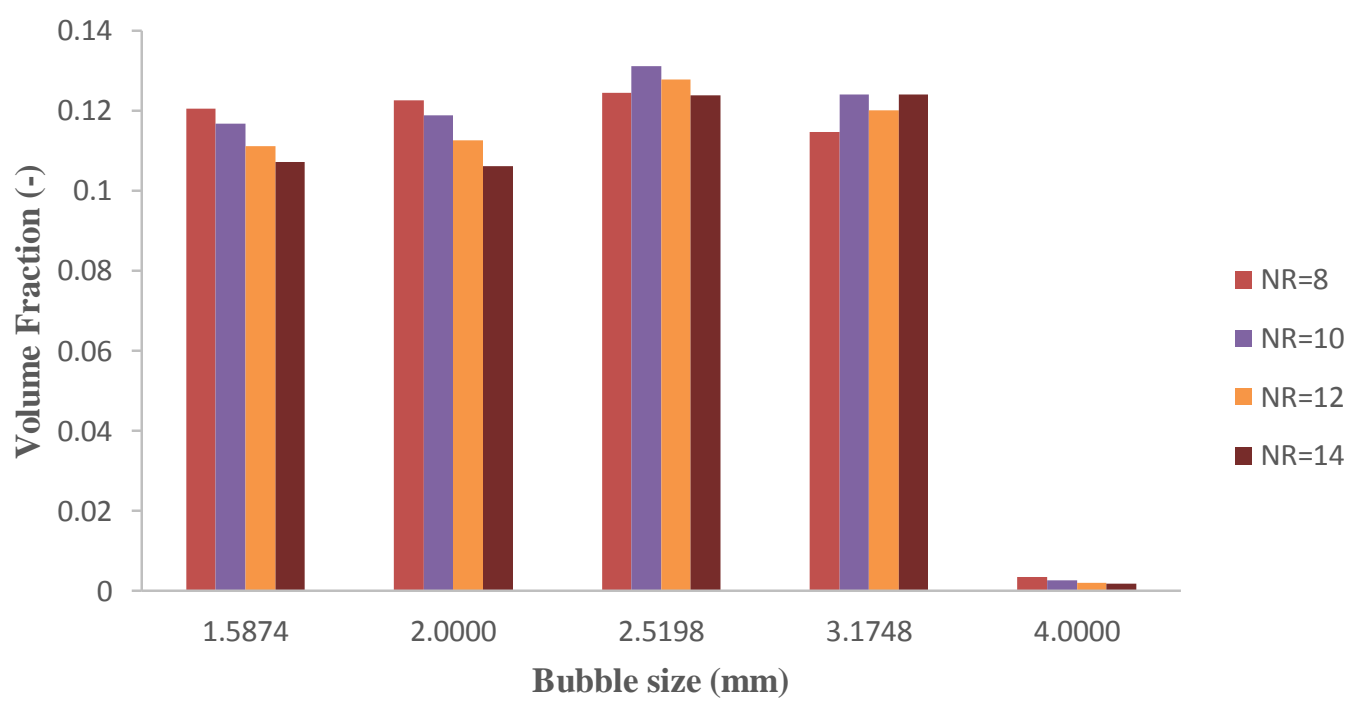

(b) Medium size bubble group

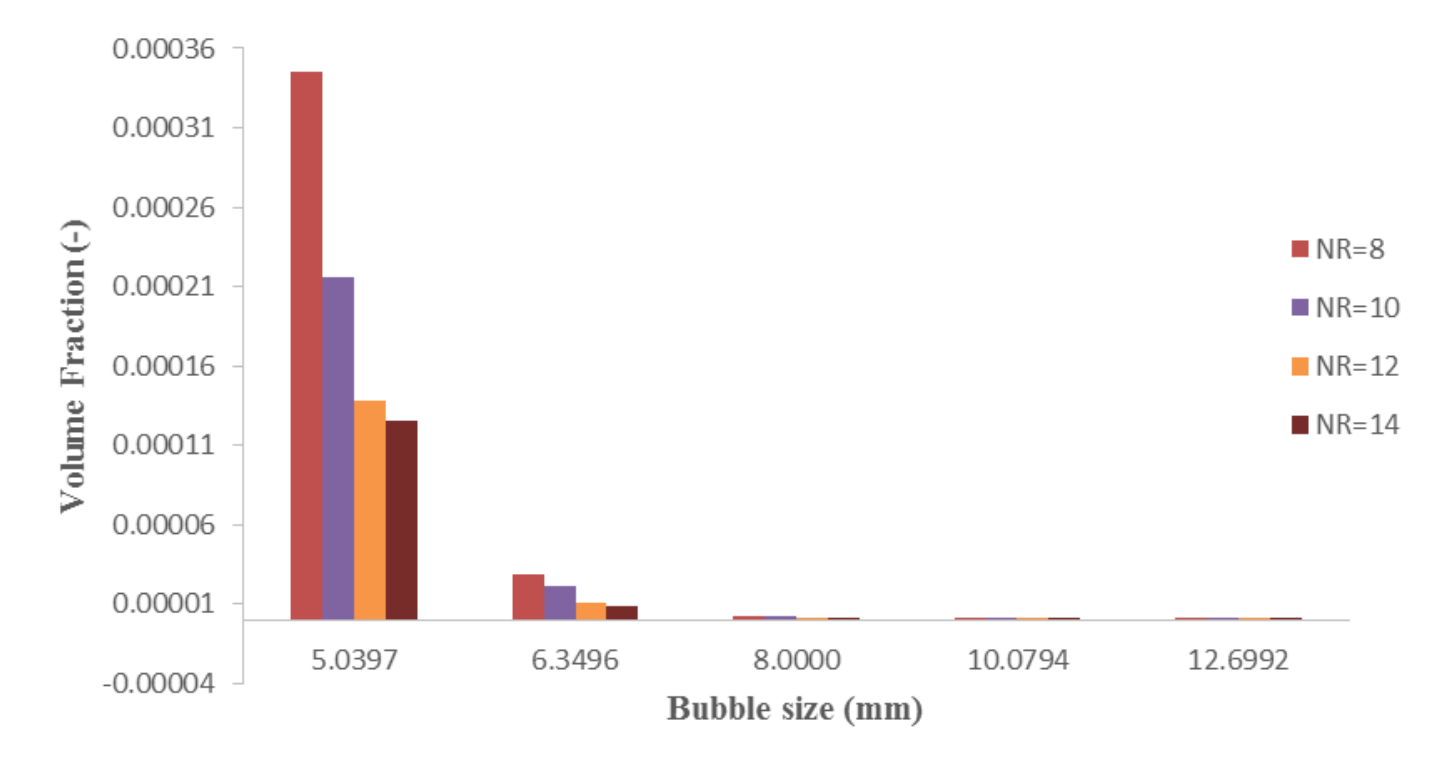

(c) Large bubble size group

Figure (5.5- 2). Effect of the speed ratio on the volume fractions of the bubbles with different sizes.

Figure 5.5-3 demonstrates that the large bubbles were observed on the central impeller blades, and the regions between the central impeller and the anchor. As it can be seen, the bubbles were 
Chapter 5: Results and Discussion

skewed towards the top and the bottom edges of the anchor blades, leading to bubble coalescence. This phenomenon was more pronounced at the speed ratios equal and lower than 10 . As it has been presented in Figure 5-5-3, the speed ratio affected the volume fractions of the large bubble group, Bin 0 to Bin 5, negatively. In fact, the fraction of the larger bubbles reduced at the higher speed ratios.

In all considered speed ratios, the bubbles with the diameter of $2.52 \mathrm{~mm}$, Bin 7, occured mostly in the upper part of the vessel, especially near the top shaft. The bubbles with the diameters less than $1.58 \mathrm{~mm}$ (Bin 10 to Bin 14) were mainly found at the bottom part of the vessel. Therefore, the bubbles getting out of the ring sparger were broken-up by the shear stress induced by the central impeller, and then dispersed in the direction of the flow generated by the upward pumping pitched blade turbine. Meanwhile, the bubble breakage largely happened when the bubbles hit the anchor blades and the vessel walls. As a result, the bubble coalescence prevailed at the upper part of the vessel, while the bubble breakage was more dominated in the lower section of the aerated coaxial tank, at which the turbulence dissipation rate reached its highest values. 
Chapter 5: Results and Discussion

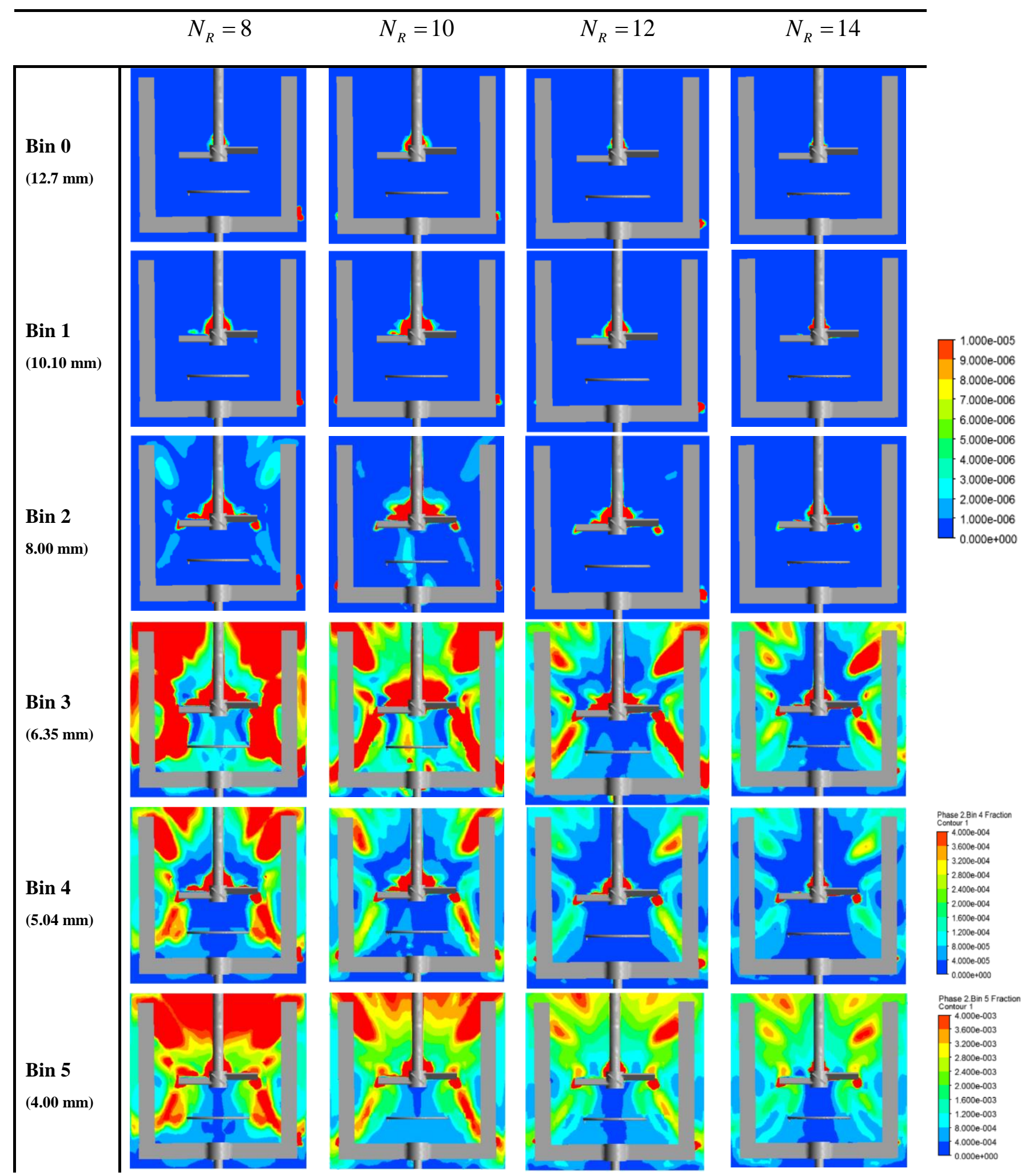


Chapter 5: Results and Discussion

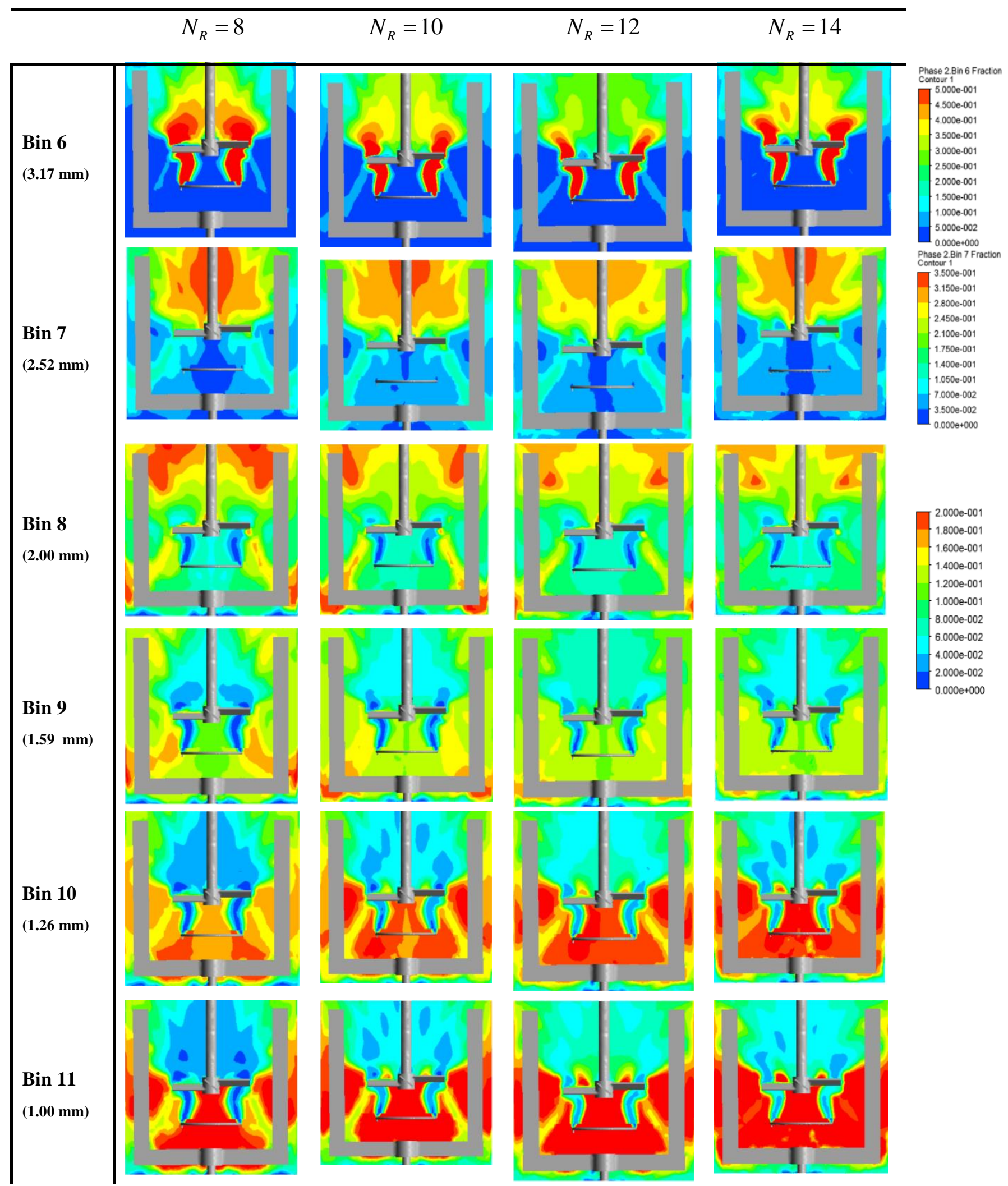




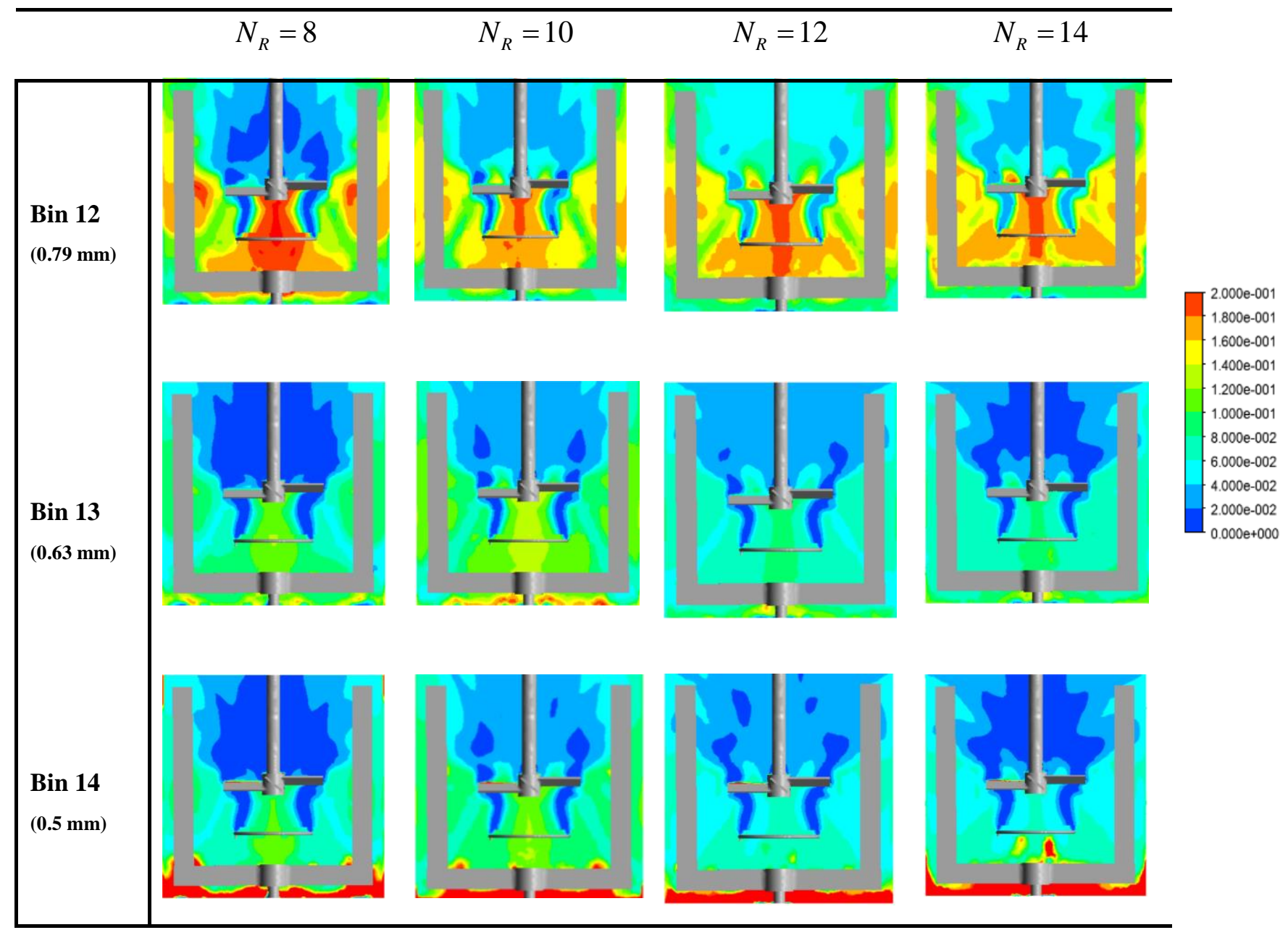

Figure (5.5- 3). Effect of the speed ratio on dispersion of bubbles with different diameters within the aerated coaxial mixer ( $\mu=0.05 \mathrm{~Pa} . \mathrm{s}, N_{c}=200 \mathrm{rpm}, N_{R}=8,10,12,14, Q_{g}=0.0283 \mathrm{~m}^{3} / \mathrm{min}$ ).

The interfacial area, which has a significant impat on the mass transfer rate, increases with a decrease in bubble sizes. Thus, in order to assess the performance of the aerated coaxial mixer in terms of its capability to generate and disperse the small bubbles, the vessel was divided into two compartments, see Figure 5.5-5. The total area-weighted volume fractions of the small bubble size groups in the upper and the bottom regions were then compared at the different speed ratios. Based on the results summarized in Table 5.5-2, the volume fractions of most of the small bubble groups, Bin 10 to Bin 14, were increased by increasing the speed ratio. In addition, it was noticed that the bubbles were dispersed more efficiently by the coaxial mixer in the upper part of the vessel at the 
Chapter 5: Results and Discussion

speed ratio of 12 . Therefore, the regions with no/less small bubbles were minimized, which is favorable in most of the gas-liquid contact systems. Overall, the performance of the coaxial mixer was enhanced with regards to the quality of the gas dispersion throughout the coaxial mixing vessel at the speed ratios higher than 10 , especially the speed ratio equal to 12. .

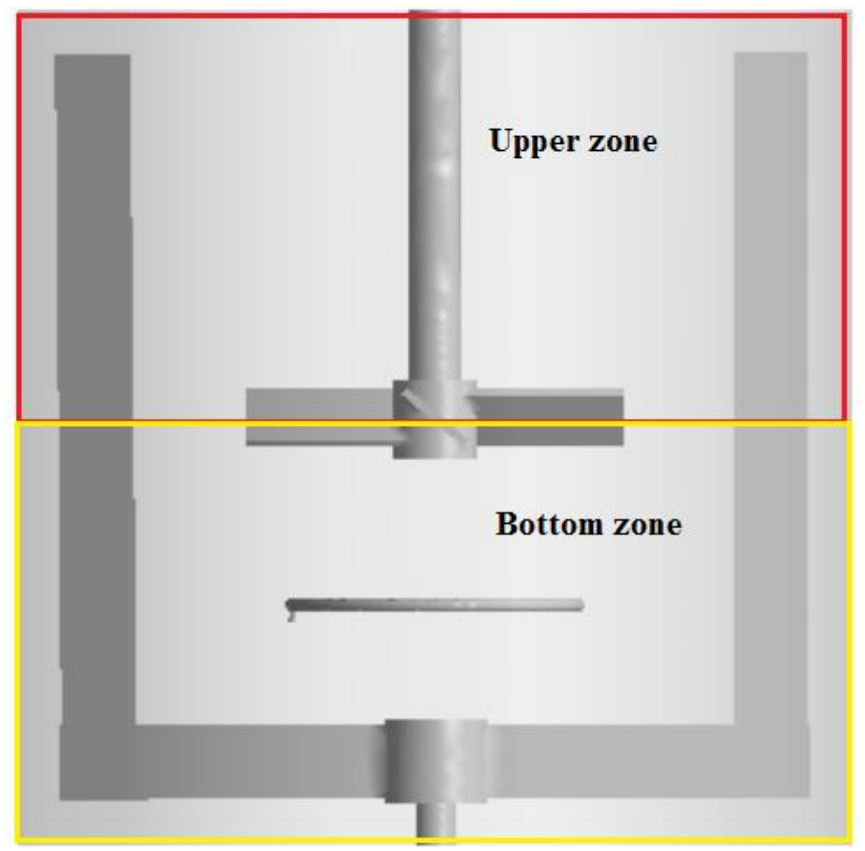

Figure (5.5- 4). Defined compartments in the coaxial mixer. 
Chapter 5: Results and Discussion

Table (5.5- 2). Effect of the speed ratio on the volume fractions of the small size bubbles in the upper and bottom compartments of the coaxial mixer.

\begin{tabular}{|c|c|c|c|c|c|c|c|c|}
\hline \multirow[b]{3}{*}{ Bubble size (m) } & \multicolumn{4}{|c|}{$\begin{array}{l}\text { Upper Compartment } \\
\text { Volume fraction }(\%)\end{array}$} & \multicolumn{4}{|c|}{$\begin{array}{l}\text { Bottom Compartment } \\
\text { Volume fraction }(\%)\end{array}$} \\
\hline & \multicolumn{4}{|c|}{ Speed ratio (-) } & \multicolumn{4}{|c|}{ Speed ratio (-) } \\
\hline & 14 & 12 & 10 & 8 & 14 & 12 & 10 & 8 \\
\hline 0.00050 (bin 14) & 3.42 & 3.70 & 3.60 & 3.90 & 13.46 & 10.94 & 9.30 & 11.22 \\
\hline 0.00063 (bin 13) & 4.00 & 4.10 & 3.40 & 3.50 & 7.50 & 7.40 & 7.23 & 8.10 \\
\hline 0.00079 (bin 12) & 8.30 & 8.40 & 7.00 & 6.70 & 13.73 & 14.13 & 13.95 & 13.80 \\
\hline 0.00100 (bin 11) & 10.98 & 10.98 & 9.40 & 8.50 & 18.19 & 18.86 & 18.76 & 16.23 \\
\hline 0.0012 (bin 10) & 9.80 & 9.90 & 9.00 & 8.80 & 16.08 & 16.66 & 16.92 & 14.58 \\
\hline
\end{tabular}

\subsubsection{Conclusion}

An Eulerian two phase model coupled with PBM was used to evaluate the bubble size distributions within the aerated coaxial mixer. In this study, the effect of the speed ratio on the bubble sizes was comprehensively investigated. The population balance model described the bubble breakage and coalescence within the aerated coaxial mixer. The experimental data of the mean bubble sizes, which were obtained from the local gas holdup values measured through ERT, were corroborated by the simulation data. These results revealed that the quality of the gas dispersion was improved at the speed ratios higher than 10. It was found that beyond the speed ratio of 10 , the volume fractions of the large bubbles were decreased while the volume fraction of the small bubbles increased. The aforementioned phenomenon was more significant at the speed 
Chapter 5: Results and Discussion

ratio of 12. Furthermore, it was noticed that the breakage and coalescence were more dominant in the bottom and top regions of the vessel, respectively. 


\section{Chapter 6}

\section{Conclusions}

The agitation of the Newtonian corn syrup solution in a novel aerated coaxial mixer was investigated in this doctoral thesis. The experimental and CFD approaches were employed to explore the mixing performance characteristics. The evaluation of the mixing performance was based on the mixing time, gassed power consumption, gas holdup, and bubble size distribution. The aerated coaxial mixer was composed of a central impeller (pitched blade turbine, upward or downward pumping) and a wall scraping anchor. The effects of the operating parameters including the central impeller type, rotation mode, speed ratio, fluid viscosity, and gas flow rate on the performance of this novel aerated coaxial mixer were analyzed.

Electrical resistance tomography, ERT, was employed to measure the degree of homogeneity, and local and global gas holdup values. In addition, the dynamic gas disengagement technique in conjunction with ERT was used to study the effect of speed ratio on the Sauter mean bubble diameter and the number of bubble size classes at different regions inside of the aerated coaxial mixer. CFD technique was utilized to simulate the three-dimensional flow field of the aerated coaxial mixer. Furthermore, the population balance model, PBM, coupled with CFD was adopted 
Chapter 6: Conclusions

to characterize the bubble size distributions within the vessel. The main findings of the current work are:

In the first stage of this thesis, for the first time, mixing characteristics of an aerated coaxial mixer composed of an anchor and a central impeller were investigated using the non-invasive flow visualization technique called electrical resistance tomography (ERT). Corn syrup solutions with different viscosity were used as the viscous Newtonian fluids. Two coaxial configurations were considered: the anchor - PBD (a pitched blade downward pumping impeller) and the anchor PBU (a pitched blade upward pumping impeller). In this study, the effects of central impeller types, speed ratios (central impeller speed/anchor speed), rotation modes, gas flow rates, and viscosity on the mixing time and power uptake were explored. In addition, the interaction effects of the most efficacious factors on the mixing time in an aerated coaxial mixer was obtained using response surface methodology (RSM).

- The mixing times obtained by the coaxial mixers were shorter than those acquired by a single impeller system.

- The PBU-anchor mixer outweighed the PBD-anchor mixer in terms of mixing time and power uptake.

- In the presence of gas, the flow pattern induced by the impeller, gas or combination of both played a significant role in affecting the mixing time. In flooding regime, gassing showed positive effect on the blending time while in loading and complete dispersion conditions, the pumping capacity of the central impeller affected by gassing and led to a rise in mixing time.

- The onset of dispersion can be affected by the central impeller type, central impeller speed, and the viscosity of the fluid being agitated. It was also found that the fluid viscosity 
Chapter 6: Conclusions

significantly affected the capability of the impeller to fully disperse the gas in the mixing vessel. At the higher fluid viscosities, the impeller can be flooded at the lower gas flow rates.

- Response surface methodology (RSM) was used to study the individual and interactive effects of the central impeller speed, the gas flow rate, the anchor speed, and viscosity on the mixing time. The developed quadratic model indicated a high coefficient of determination. It was found that the viscosity was the most significant factor while the gas flow rate was the least effective one. Considering the interaction effects, the central impeller speed and the gas flow rate showed the highest effect on the mixing time.

For the first time ERT was employed to characterize the effects of the central impeller speed, anchor speed, and speed ratio on the radial distribution of the sparged gas within the aerated coaxial mixing system. Furthermore, in order to overcome the problems associated with the prediction of the power uptake of the aerated coaxial mixer, two novel dimensional and dimensionless correlations were proposed.

- In order to characterize the power consumption of an aerated coaxial mixing vessel consisting of a close clearance anchor and a central impeller, novel correlations for the gas flow number and power number were developed to obtain a master power curve for the aerated coaxial mixing vessel.

- The experimental results demonstrated that the gassed power of the aerated coaxial mixing $\operatorname{vessel}\left(P_{g}\right)$ was dependent on the central impeller type, central impeller speed, anchor speed, speed ratio, gas flow rate, and the ungassed power uptake of the agitators. Accordingly, a dimensional correlation was proposed for the estimation of the aerated power uptake of the coaxial mixers as a function of the abovementioned design parameters. 
Chapter 6: Conclusions

- The effect of the sparged gas on the power uptake of the central impeller and the wall scraping anchor was completely different.

- At a relatively low fluid viscosity, the power consumption of the anchor decreased with an increase in the speed ratio. However, at a higher fluid viscosity and beyond the critical speed ratio equal to 10 , the anchor power increased by increasing the speed ratio (i.e. decreasing the anchor speed).

For the first time, the global and local gas holdup values and the Sauter mean bubble diameter for an aerated coaxial mixing vessel equipped with a central pitched blade turbine and an anchor impeller were estimated for the viscous corn syrup solution. The main characteristics of the bubbles including the number of bubble size classes and the contribution of each class of bubble in the overall gas holdup were measured by using the dynamic gas disengagement theory coupled with the electrical resistance tomography (ERT) data. The impacts of the speed ratio and the gas flow rate on the bubble behavior (size and dispersion) were also explored.

- At the fixed central impeller speed and the gas velocity, the number of bubble size classes, which were identified at two different heights of the aerated coaxial mixing vessel, was not varied by changing the speed ratio.

- The percentage of the overall gas holdup, which was occupied by each class of bubbles, was reduced by increasing the speed ratio at a constant gas velocity.

- The local gas holdups measured in the vicinity of the vessel wall were increased as the speed ratio increased up to the critical value of 10 . However, by further increase of the speed ratio, the local gas holdup was affected inversely.

- The minimum gas holdup was achieved at the speed ratio of 10 at the different gas velocities employed in this study. 


\section{Chapter 6: Conclusions}

For the first time, a CFD model was developed for the simulation of the aerated PBU-anchor coaxial mixing to investigate the effects of the speed ratio, rotation modes (co-rotating and counter-rotating), and fluid viscosity on the local and global gas holdup values, flow pattern within the vessel, and turbulent kinetic energy. To validate the developed model, simulated gas holdup and gassed power uptake were compared with the experimental values. To gather the experimental gas holdup values, the electrical resistance tomography technique was used. The main findings were as follows:

- From both experimental and simulation studies, it was found that the highest gas volume fractions were attained at the speed ratios higher than 10 led regardless of the fluid viscosity.

- The shape of the vortex generated by the anchor blades was not affected by the anchor speed; however, the size of the vortex was strongly dependent on the anchor speed.

- The co-rotating coaxial mixer outweighed the counter-rotating one in term of gas dispersion.

- The counter-rotating coaxial mixer was more efficient at the speed ratios lower than 10.

- The turbulent kinetic energy attained in the counter-rotating mode was lower than those for the co-rotating coaxial mixer in the most regions within the mixing tank, especially near the vessel walls.

- The size and the number of circulation loops developed within the coaxial mixer were affected by the speed ratio.

$>$ In this study, for the first time, the CFD model was coupled with the population balance model (PBM) to investigate the bubble size distribution for the aerated PBU-anchor coaxial mixer. The MUSIG was employed to incorporate the bubble breakup and coalecencse into the model. The CFD-PBM model was then utilized to study the effect of the speed ratio on the bubble sizes The significant outcomes of this research were as follows: 
Chapter 6: Conclusions

- It was found that beyond the speed ratio of 10, the volume fractions of the large bubbles were decreased while the volume fraction of the small bubbles increased.

- At speed ratio of 12 , the regions with no/less small bubbles were minimized.

- It was noticed that breakage and coalescence were more dominant in the bottom and top regions of the vessel, respectively.

\subsection{Recommendations for Future Work}

The experimental and numerical results of this study drew attention to the following areas for future considerations:

- The performance of the aerated coaxial mixing vessel in the agitation of highy viscous nonNewtonian fluids should be investigated.

- The effect of other types of central impellers, bottom clearance of the central impeller, different distances between the central impeller and the sparger, and various types of the gas spargers on the performance of the aerated coaxial mixer should be assessed.

- The efficiency of the aerated coaxial mixers composed of double central impellers (identical/different) should be analyzed

- Different drag models should be examined in order to increase the accuracy of the developed CFD model.

- The bubble size distribution and the Sauter mean bubble diameter as a function of the operating conditions and design parameters should be measured for the aerated coaxial mixers using the advanced endoscopy technique. 


\section{References}

Abdullah, B., Dave, C., Nguyen, T.H., Cooper, C.G. and Adesina, A.A., 2011. Electrical resistance tomography-assisted analysis of dispersed phase hold-up in a gas-inducing mechanically stirred vessel. Chemical engineering science, 66(22), pp.5648-5662.

Abrahamson, J., 1975. Collision rates of small particles in a vigorously turbulent fluid. Chemical Engineering Science, 30(11), pp.1371-1379.

Abrardi, V., Rovero, G., Baldi, G., Sicardi, S. and Conti, R., 1990. Hydrodynamics of a gas-liquid reactor stirred with a multi-impeller system. Chemical engineering research \& design, 68(6), pp.516-522.

Alves, S.S., Maia, C.I., Vasconcelos, J.M.T. and Serralheiro, A.J., 2002. Bubble size in aerated stirred tanks. Chemical Engineering Journal, 89(1), pp.109-117.

Andrew, PS. Sydney, Gas analysis, U.S. Patent No. 4,328,780, 1982.

Arjunwadkar, S.J., Saravanan, K., Pandit, A.B. and Kulkarni, P.R., 1998. Optimizing the impeller combination for maximum hold-up with minimum power consumption. Biochemical engineering journal, 1(1), pp.25-30.

Arrua, L.A., McCoy, B.J. and Smith, J.M., 1990. Gas-liquid mass transfer in stirred tanks. AIChE journal, 36(11), pp.1768-1772.

Ascanio, G., Brito-Bazán, M., La Fuente, E.B.D., Carreau, P.J. and Tanguy, P.A., 2002. Unconventional configuration studies to improve mixing times in stirred tanks. Canadian journal of chemical engineering, 80(4), pp.558-565.

Aubin, J., Mavros, P., Fletcher, D.F., Bertrand, J. and Xuereb, C., 2001. Effect of axial agitator configuration (up-pumping, down-pumping, reverse rotation) on flow patterns generated in stirred vessels. Chemical Engineering Research and Design, 79(8), pp.845-856.

Ayeni, O.O., Wu, C.L., Nandakumar, K. and Joshi, J.B., 2016. Development and validation of a new drag law using mechanical energy balance approach for DEM-CFD simulation of gas-solid fluidized bed. Chemical Engineering Journal, 302, pp.395-405.

Azargoshasb, H., Mousavi, S.M., Jamialahmadi, O., Shojaosadati, S.A. and Mousavi, S.B., 2016. Experiments and a three-phase computational fluid dynamics (CFD) simulation coupled with population balance equations of a stirred tank bioreactor for high cell density cultivation. The Canadian Journal of Chemical Engineering, 94(1), pp.20-32.

Babaei, R., Bonakdarpour, B. and Ein-Mozaffari, F., 2015a. Analysis of gas phase characteristics and mixing performance in an activated sludge bioreactor using electrical resistance tomography. Chemical Engineering Journal, 279, pp.874-884.

Babaei, R., Bonakdarpour, B. and Ein-Mozaffari, F., 2015b. The use of electrical resistance tomography for the characterization of gas holdup inside a bubble column bioreactor containing activated sludge. Chemical Engineering Journal, 268, pp.260-269. 
Bakker, A. and Gates, L.E., 1995. Properly choose mechanical agitators for viscous liquids. Chemical engineering progress, 91(12), pp.25-34..

Bakker, A., 1992. Hydrodynamics of stirred gas-liquid dispersions. Ph.D. Thesis, Delft University of Technology, The Netherlands.

Bao, Y., Yang, B., Xie, Y., Gao, Z., Zhang, Z., Liu, T. and Gao, X., 2011. Power demand and mixing performance of coaxial mixers in non-Newtonian fluids. Journal of chemical engineering of Japan, 44(2), pp.57-66.

Barigou, M. and Greaves, M., 1991. A capillary suction prove for bubble size measurement. Measurement Science and Technology, 2(4), pp.318.

Bird, R.B., Stewart, W.E. and Lightfoot, E.N., 2004. Transport phenomena. 2002. John Wiley \& Sons, New York.

Blakebrough, N. and Sambamurthy, K., 1966. Mass transfer and mixing rates in fermentation vessels. Biotechnology and Bioengineering, 8(1), pp.25-42.

Bonnot, S., Cabaret, F., Fradette, L. and Tanguy, P.A., 2007. Characterization of mixing patterns in a coaxial mixer. Chemical Engineering Research and Design, 85(8), pp.1129-1135.

Bouaifi, M., \& Roustan, M., 2001. Power consumption, mixing time and homogenisation energy in dual-impeller agitated gas-liquid reactors. Chemical Engineering and Processing: Process Intensification, 40(2), 87-95.

Boussinesq, J., 1877. Essai sur la théorie des eaux courantes. Imprimerie nationale.

Boyer, C., Duquenne, A. M., Wild, G., 2002. Measuring techniques in gas-liquid and gas-liquidsolid reactors. Chemical Engineering Science, 57(16), pp. 3185-3215.

Brennan, D.J. and Lehrer, I.H., 1976. Impeller mixing in vessels experimental studies on the influence of some parameters and formulation of a general mixing time equation. Trans. Inst. Chem. Eng, 54, pp.139-152.

Brucato, A., Grisafi, F. and Montante, G., 1998. Particle drag coefficients in turbulent fluids. Chemical Engineering Science, 53(18), pp.3295-3314.

Bujalski, W., Jaworski, Z. and Nienow, A.W., 2002. CFD study of homogenization with dual Rushton turbines-Comparison with experimental results: Part II: The multiple reference frame. Chemical Engineering Research and Design, 80(1), pp.97-104.

Busciglio, A., Grisafi, F., Scargiali, F. and Brucato, A., 2013. On the measurement of local gas hold-up, interfacial area and bubble size distribution in gas-liquid contactors via light sheet and 
image analysis: Imaging technique and experimental results. Chemical Engineering Science, 102, pp.551-566.

Cabaret, F., Fradette, L. and Tanguy, P.A., 2008. Gas-liquid mass transfer in unbaffled dualimpeller mixers. Chemical Engineering Science, 63(6), pp.1636-1647.

Calderbank, P.H. and Moo-Young, M.B., 1959. The prediction of power consumption in the agitation of non-Newtonian fluids. Trans. Inst. Chem. Eng, 37(3), pp.26-33.

Calderbank, P.H., 1958. Physical rate processes in industrial fermentation. Part I: The interfacial area in gas-liquid contacting with mechanical agitation. Trans. Inst. Chem. Eng, 36(5), pp.433440.

Chaouki, J., Larachi, F. and Duduković, M.P., 1997. Noninvasive tomographic and velocimetric monitoring of multiphase flows. Industrial \& engineering chemistry research, 36(11), pp.44764503.

Cheng, J. and Carreau, P.J., 1994. Aerated mixing of viscoelastic fluids with helical ribbons impellers. Chemical engineering science, 49(12), pp.1965-1972.

Chhabra, R.P. and Richardson, J.F., 1999. Non-Newtonian flow in the process industries: fundamentals and engineering applications. Butterworth-Heinemann.

Chung, K.H.K., Simmons, M.J.H. and Barigou, M., 2009. Local gas and liquid phase velocity measurement in a miniature stirred vessel using PIV combined with a new image processing algorithm. Experimental Thermal and Fluid Science, 33(4), pp.743-753.

Cooke, M., Middleton, J.C. and Bush, J.R., 1988. Mixing and mass transfer in filamentous fermentations. In Proceedings of the 2nd International Conference on Bioreactor Fluid Dynamics, pp. 37-64. Elsevier Applied Science Publishers: Amsterdam.

Cooke, M., Heggs, P.J. and Rodgers, T.L., 2008. The effect of solids on the dense phase gas fraction and gas-liquid mass transfer at conditions close to the heterogeneous regime in a mechanically agitated vessel. chemical engineering research and design, 86(8), pp.869-882.

Cronin, D.G., 1992. An experimental study of the mixing in a proto-fermenter agitated by dual Rushton turbines. Trans. Inst. Chem. Eng., PartC, 72, pp.35-40.

Cui, Y.Q., Van der Lans, R.G.J.M. and Luyben, K.C.A., 1996. Local power uptake in gas-liquid systems with single and multiple Rushton turbines. Chemical engineering science, 51(11), pp.2631-2636. 
De Jesus, S.S., Neto, J.M. and Maciel Filho, R., 2017. Hydrodynamics and mass transfer in bubble column, conventional airlift, stirred airlift and stirred tank bioreactors, using viscous fluid: A comparative study. Biochemical Engineering Journal, 118, pp.70-81.

Delaplace, G., Leuliet, J.C. and Relandeau, V., 2000. Circulation and mixing times for helical ribbon impellers. Review and experiments. Experiments in fluids, 28(2), pp.170-182.

Dhanasekharan, K.M., Sanyal, J., Jain, A. and Haidari, A., 2005. A generalized approach to model oxygen transfer in bioreactors using population balances and computational fluid dynamics. Chemical Engineering Science, 60(1), pp.213-218.

Dickey, D.S., 1979. Turbine Agitated Gas Dispersion-Power, Flooding and Hold-up. In Preprints of the 72nd Annual Meeting of AIChE, p. 116d, San Francisco, USA.

Einsele, A. and Finn, R.K., 1980. Influence of gas flow rates and gas holdup on blending efficiency in stirred tanks. Industrial \& Engineering Chemistry Process Design and Development, 19(4), pp.600-603.

Espinosa-Solares, T., Brito-De la Fuente, E., Tecante, A., Medina-Torres, L. and Tanguy, P.A., 2002. Mixing time in rheologically evolving model fluids by hybrid dual mixing systems. Chemical Engineering Research and Design, 80(8), pp.817-823.

Farhat, M., Rivera, C., Fradette, L., Heniche, M. and Tanguy, P.A., 2007. Numerical and experimental study of a dual-shaft coaxial mixer with viscous fluids. Industrial \& engineering chemistry research, 46(14), pp.5021-5031..

Fischer, J., Brüring, S., \& Lübbert, A., 1992. Gas-phase properties in stirred tank bioreactors. Chemical engineering \& technology, 15(6), pp. 390-394.

Ford, J. J., Heindel, T. J., Jensen, T. C., \& Drake, J. B., 2008. X-ray computed tomography of a gas-sparged stirred-tank reactor. Chemical Engineering Science 63(8), pp. 2075-2085.

Foucault, S., Ascanio, G., \& Tanguy, P. A., 2004. Coaxial mixer hydrodynamics with Newtonian and non-Newtonian fluids. Chemical engineering \& technology, 27(3), pp. 324-329.

Foucault, S., Ascanio, G., \& Tanguy, P. A., 2005. Power characteristics in coaxial mixing: Newtonian and non-Newtonian fluids. Industrial \& engineering chemistry research 44(14), pp. 5036-5043.

Foucault, S., Ascanio, G., Tanguy, P. A., 2006. Mixing times in coaxial mixers with Newtonian and non-Newtonian fluids. Industrial \& engineering chemistry research 45(1), pp. 352-359. 
Fradette, L., Tanguy, P.A., Bertrand, F., Thibault, F., Ritz, J.B. and Giraud, E., 2007. CFD phenomenological model of solid-liquid mixing in stirred vessels. Computers \& chemical engineering, 31(4), pp.334-345.

Fransolet, E., Crine, M., L'Homme, G., Toye, D., Marchot, P., 2001. Analysis of electrical resistance tomography measurements obtained on a bubble column. Measurement Science and Technology 12(8), pp. 1055.

Gill, N. K., Appleton, M., Baganz, F., Lye, G. J., 2008. Quantification of power consumption and oxygen transfer characteristics of a stirred miniature bioreactor for predictive fermentation scaleup. Biotechnology and Bioengineering 100(6), pp. 1144-1155.

Gogate, P.R., Beenackers, A.A. and Pandit, A.B., 2000. Multiple-impeller systems with a special emphasis on bioreactors: a critical review. Biochemical Engineering Journal, 6(2), pp.109-144.

Grace, J.R., Wairegi, T. and Nguyen, T.H., 1976. Shapes and velocities of single drops and bubbles moving freely through immiscible liquids. Trans. Inst. Chem. Eng, 54(3), pp.167-173.

Greaves, M., \& Kobbacy, K. A. H., 1984. Measurement of bubble size distribution in turbulent gas-liquid dispersions. Chemical Engineering Research and Design 62(1), pp. 3-12.

Grenville, R.K., Nienow, A.W., 2004. Blending of miscible liquids Chapter 9. Paul, E. L., AtiemoObeng, V.A., Kresta, S.M. (Eds.), "Handbook of Industrial Mixing: Science and Practice". WileyInterscience, Hoboken, NJ

Grenville, R., Ruszkowski, S. and Garred, E., 1995, June. Blending of miscible liquids in the turbulent and transitional regimes. In Mixing XV, 15th Biennial North American Mixing Conference, Banff, AL, Canada.

Guillard, F., Trägårdh, C., \& Fuchs, L., 2000. A study on the instability of coherent mixing structures in a continuously stirred tank. Chemical Engineering Science, 55 (23), pp. 5657-5670.

Hadjiev, D., Sabiri, N. E., \& Zanati, A., 2006. Mixing time in bioreactors under aerated conditions. Biochemical engineering journal, 27(3), pp. 323-330.

Halow, J.S., 1995. Capacitance imaging of fluidized beds. Process Tomography--Principles, Techniques and Applications, edited by RA Williams and MS Beck, Butterworth Heinemann, pp.447-486.

Hamood-ur-Rehman, M., Dahman, Y., Ein-Mozaffari, F., 2012. Investigation of mixing characteristics in a packed-bed external loop airlift bioreactor using tomography images. Chemical Engineering Journal 213, pp. 50-61. 
Hashemi, N., Ein-Mozaffari, F., Upreti, S.R. and Hwang, D.K., 2016a. Analysis of mixing in an aerated reactor equipped with the coaxial mixer through electrical resistance tomography and response surface method. Chemical Engineering Research and Design, 109, pp.734-752.

Hashemi, N., Ein-Mozaffari, F., Upreti, S.R. and Hwang, D.K., 2016b. Analysis of power consumption and gas holdup distribution for an aerated reactor equipped with a coaxial mixer: Novel correlations for the gas flow number and gassed power. Chemical Engineering Science, 151, pp.25-35.

Hashemi, N., Ein-Mozaffari, F., Upreti, S.R. and Hwang, D.K., 2016c. Experimental investigation of the bubble behavior in an aerated coaxial mixing vessel through electrical resistance tomography (ERT). Chemical Engineering Journal, 289, pp.402-412.

Hass, V.C. and Nienow, A.W., 1989. Chem.Ing.Tech., 61, No.2, 152-154

Hassan, I. and Robinson, C.W., 1977. Stirred-tank mechanical power requirement and gas holdup in aerated aqueous phases. AIChE Journal, 23(1), pp.48-56.

Harris, C.K., Roekaerts, D., Rosendal, F.J.J., Buitendijk, F.G.J., Daskopoulos, P., Vreenegoor, A.J.N. and Wang, H., 1996. Computational fluid dynamics for chemical reactor engineering. Chemical Engineering Science, 51(10), pp.1569-1594.

Hicks, R.W. and Gates, L.E., 1976. How to select turbine agitators for dispersing gas into liquids. Chemical Engineering, 83(15), pp.141-148.

Higashitani, K., Yamauchi, K., Matsuno, Y. and HOSOKAWA, G., 1983. Turbulent coagulation of particles dispersed in a viscous fluid. Journal of chemical engineering of Japan, 16(4), pp.299304.

Hirata, Y., Nienow, A.W. and Moore, I.P., 1994. Estimation of cavern sizes in a shear-thinning plastic fluid agitated by a Rushton turbine based on LDA measurements. Journal of chemical engineering of Japan, 27(2), pp.235-237.

Hughmark, G.A., 1980. Power requirements and interfacial area in gas-liquid turbine agitated systems. Industrial \& Engineering Chemistry Process Design and Development, 19(4), pp.638641.

Ishii, M. and Zuber, N., 1979. Drag coefficient and relative velocity in bubbly, droplet or particulate flows. AIChE Journal, 25(5), pp.843-855.

Jahoda, M., Tomášková, L. and Moštěk, M., 2009. CFD prediction of liquid homogenisation in a gas-liquid stirred tank. Chemical Engineering Research and Design, 87(4), pp.460-467. 
Jin, H., Wang, M. and Williams, R.A., 2007. Analysis of bubble behaviors in bubble columns using electrical resistance tomography. Chemical Engineering Journal, 130(2), pp.179-185.

John, A., 1998. A novel reactor with two independently-driven impellers for gas-liquid processing (Doctoral dissertation, University of Birmingham).

Junker, B.H., Stanik, M., Barna, C., Salmon, P., Paul, E. and Buckland, B.C., 1998. Influence of impeller type on power input in fermentation vessels. Bioprocess and Biosystems Engineering, 18(6), pp.401-412.

Li, H. and Prakash, A., 2000. Influence of slurry concentrations on bubble population and their rise velocities in a three-phase slurry bubble column. Powder Technology, 113(1), pp.158-167.

Kazemzadeh, A., Ein-Mozaffari, F., Lohi, A. and Pakzad, L., 2016a. Investigation of hydrodynamic performances of coaxial mixers in agitation of yield-pseudoplasitc fluids: Single and double central impellers in combination with the anchor. Chemical Engineering Journal, 294, pp.417-430.

Kazemzadeh, A., Ein-Mozaffari, F., Lohi, A. and Pakzad, L., 2016b. A new perspective in the evaluation of the mixing of biopolymer solutions with different coaxial mixers comprising of two dispersing impellers and a wall scraping anchor. Chemical Engineering Research and Design, 114, pp.202-219.

Kazemzadeh, A., Ein-Mozaffari, F., Lohi, A. and Pakzad, L., 2017. Intensification of mixing of shear-thinning fluids possessing yield stress with the coaxial mixers composed of two different central impellers and an anchor. Chemical Engineering and Processing: Process Intensification. http://dx.doi.org/10.1016/j.cep.2016.10.019

Kerdouss, F., Bannari, A. and Proulx, P., 2006. CFD modeling of gas dispersion and bubble size in a double turbine stirred tank. Chemical Engineering Science, 61(10), pp.3313-3322.

Khopkar, A. R., Rammohan, A. R., Ranade, V. V., \& Dudukovic, M. P., 2005. Gas-liquid flow generated by a Rushton turbine in stirred vessel: CARPT/CT measurements and CFD simulations. Chemical Engineering Science, 60(8), pp. 2215-2229.

Khopkar, A. R., Fradette, L., \& Tanguy, P. A., 2007. Hydrodynamics of a dual shaft mixer with Newtonian and non-Newtonian fluids. Chemical Engineering Research and Design, 85(6), pp. 863-871.

Khopkar, A. R., \& Tanguy, P. A., 2008. CFD simulation of gas-liquid flows in stirred vessel equipped with dual rushton turbines: influence of parallel, merging and diverging flow configurations. Chemical engineering science, 63(14), pp. 3810-3820. 
Köhler, S. and Hemmerle, W., 2003. Analysis of the power characteristic of a coaxial agitator with varied diameter and speed ratio of inner and outer mixing device. In Proceedings of the 11th European Conference on Mixing (pp. 14-17). VDI-Gesellschaft Verfahrenstechnik und Chemieingenieurwesen (VDI GVC), Berlin.

Kraume, M. and Zehner, P., 2001. Experience with experimental standards for measurements of various parameters in stirred tanks: A comparative test. Chemical Engineering Research and Design, 79(8), pp.811-818.

Kumar, A., Degaleesan, T.E., Laddha, G.S. and Hoelscher, H.E., 1976. Bubble swarm characteristics in bubble columns. The Canadian Journal of Chemical Engineering, 54(6), pp.503508.

Kumar, S. B., Moslemian, D., \& Duduković, M. P., 1995. A $\gamma$-ray tomographic scanner for imaging voidage distribution in two-phase flow systems. Flow Measurement and Instrumentation, 6(1), pp. 61-73.

Kumaresan, T., \& Joshi, J. B., 2006. Effect of impeller design on the flow pattern and mixing in stirred tanks. Chemical Engineering Journal, 115 (3), pp. 173-193.

Kuncewicz, C., \& Pietrzykowski, M., 2001. Hydrodynamic model of a mixing vessel with pitchedblade turbines. Chemical engineering science, 56(15), pp. 4659-4672.

Kuzmanić, N., \& Ljubičić, B., 2001. Suspension of floating solids with up-pumping pitched blade impellers; mixing time and power characteristics. Chemical Engineering Journal, 84 (3), pp. 325333.

Laakkonen, M., Honkanen, M., Saarenrinne, P. and Aittamaa, J., 2005. Local bubble size distributions, gas-liquid interfacial areas and gas holdups in a stirred vessel with particle image velocimetry. Chemical Engineering Journal, 109(1), pp.37-47.

Laakkonen, M., Moilanen, P., Alopaeus, V. and Aittamaa, J., 2007. Modelling local bubble size distributions in agitated vessels. Chemical Engineering Science, 62(3), pp.721-740.

Labík, L., Vostal, R., Moucha, T., Rejl, F., \& Kordač, M., 2014. Volumetric mass transfer coefficient in multiple-impeller gas-liquid contactors. Scaling-up study for various impeller types. Chemical Engineering Journal, 240, pp. 55-61

Lagisetty, J.S., Das, P.K., Kumar, R. and Gandhi, K.S., 1986. Breakage of viscous and nonNewtonian drops in stirred dispersions. Chemical Engineering Science, 41(1), pp.65-72.

Lamberto, D. J., Muzzio, F. J., Swanson, P. D., \& Tonkovich, A. L., 1996. Using time-dependent RPM to enhance mixing in stirred vessels. Chemical Engineering Science, 51(5), pp. 733-741. 
Lane, G. L., Schwarz, M. P., \& Evans, G. M., 2002. Predicting gas-liquid flow in a mechanically stirred tank. Applied Mathematical Modelling, 26(2), pp. 223-235.

Langheinrich, C., Nienow, A.W., Stevenson, N., Emery, A.N., Clayton, T.H. and Slater, N.K.H., 1995. Liquid homogenisation studies in stirred bioreactors under animal cell culture conditions. IX Eng. Found. Conf. Biochem. Eng. Davos, Switzerland, May

Lee, R. E., Finch, C. R., \& Wooledge, J. D., 1957. Mixing of high viscosity Newtonian and nonNewtonian fluids. Industrial \& Engineering Chemistry, 49 (11), pp. 1849-1854.

Lee, K.C. and Yianneskis, M., 1997. A liquid crystal thermographic technique for the measurement of mixing characteristics in stirred vessels. Chemical Engineering Research and Design, 75(8), pp.746-754.

Linek, V., Moucha, T., Sinkule, J., 1996. Gas-liquid mass transfer in vessels stirred with multiple impellers-I. Gas-liquid mass transfer characteristics in individual stages. Chemical Engineering Science 51(12), pp. 3203-3212.

Liu, B., Liu, J., Zhang, Y., Chen, M., Qin, F., Jin, Z., 2013. Experimental Research on the Power Consumption of a Coaxial Mixer in a Fluid with High Viscosity. Industrial \& Engineering Chemistry Research 52(20), pp. 6862-6867.

Liu, Y.S., Wu, J.Y. and Ho, K.P., 2006. Characterization of oxygen transfer conditions and their effects on Phaffia rhodozyma growth and carotenoid production in shake-flask cultures. Biochemical engineering journal, 27(3), pp.331-335.

Loiseau, B., Midoux, N. and Charpenntier, J.C., 1977. Some hydrodynamics and power input data in mechanically agitated gas-liquid contactors. AIChE Journal, 23(6), pp.931-935.

Luo, J.Y., Gosman, A.D., Issa, R.I., Middleton, J.C. and Fitzgerald, M.K., 1993. Full flow field computation of mixing in baffled stirred vessels. Chemical engineering research \& design, 71(3), pp.342-344.

Luong, H.T. and Volesky, B., 1979. Mechanical power requirements of gas-liquid agitated systems. AIChE Journal, 25(5), pp.893-895.

Machon, V. and Jahoda, M., 2000. Liquid Homogenization in Aerated Multi-Impeller Stirred Vessel. Chemical engineering \& technology, 23(10), pp.869-876.

Machon, V., Pacek, A.W. and Nienow, A.W., 1997. Bubble sizes in electrolyte and alcohol solutions in a turbulent stirred vessel. Chemical Engineering Research and Design, 75(3), pp.339348. 
Mann, R., Dickin, F. J., Wang, M., Dyakowski, T., Williams, R. A., Edwards, R. B., Holden, P. J., 1997a. Application of electrical resistance tomography to interrogate mixing processes at plant scale. Chemical Engineering Science 52(13), pp. 2087-2097.

Mann, R., Williams, R. A., Dyakowski, T., Dickin, F. J., Edwards, R. B., 1997b. Development of mixing models using electrical resistance tomography. Chemical Engineering Science 52(13), pp. 2073-2085.

Mann, R., Wang, M., Forrest, A. E., Holden, P. J., Dyakowski, T., DICKIN, F., Edwards, R. B., 1999. Gas-liquid and miscible liquid mixing in a plant-scale vessel monitored using electrical resistance tomography. Chemical Engineering Communications 175(1), pp. 39-48.

Markopoulos, J. and Pantuflas, E., 2001. Power Consumption in Gas-Liquid Contactors Agitated by Double-Stage Rushton Turbines. Chemical engineering \& technology, 24(11), pp.1147-1150.

Martín, M., Montes, F. J., \& Galán, M. A., 2008. Influence of impeller type on the bubble breakup process in stirred tanks. Industrial \& Engineering Chemistry Research 47(16), pp. 6251-6263.

Maxwell, J. C., 1881. A treatise on electricity and magnetism (Vol. 1). Clarendon press.

McFarlane, C.M. and Nienow, A.W., 1996. Studies of high solidity ratio hydrofoil impellers for aerated bioreactors. 4. Comparison of impeller types. Biotechnology progress, 12(1), pp.9-15.

Meister, D., Post, T., Dunn, I.J. and Bourne, J.R., 1979. Design and characterization of a multistage, mechanically stirred column absorber. Chemical Engineering Science,34(12), pp.1367-1374.

Merchuk, J. C., Contreras, A., Garcia, F., \& Molina, E., 1998. Studies of mixing in a concentric tube airlift bioreactor with different spargers. Chemical Engineering Science, 53 (4), pp. 709-719.

Metzner, A. B., \& Otto, R. E., 1957. Agitation of non-Newtonian fluids. AIChE Journal, 3(1), 310 .

Michel, B. J., Miller, S. A., 1962. Power requirements of gas-liquid agitated systems. AIChE Journal 8(2), pp. 262-266.

Micheau, F., Xuereb, C., Eyssautier, B., \& Riba, J. P., 1995. Non-Newtonian viscous liquids mixed and aerated by a double helical ribbon. Chemical Engineering Communications, 136 (1), pp. 143159.

Middleton, J. C., \& Smith, J. M., 2004. Gas-liquid mixing in turbulent systems. Handbook of Industrial Mixing: Science and Practice, pp. 585-638.

Miller, D.N., 1974. Scale-up of agitated vessels gas-liquid mass transfer. AIChE Journal, 20(3), pp.445-453. 
Montante, G., Horn, D. and Paglianti, A., 2008. Gas-liquid flow and bubble size distribution in stirred tanks. Chemical engineering science, 63(8), pp.2107-2118.

Moo-Young, M. and Blanch, H.W., 1981. Design of biochemical reactors mass transfer criteria for simple and complex systems. In Reactors and Reactions (pp. 1-69). Springer Berlin Heidelberg.

Moucha, T., Linek, V., Erokhin, K., Rejl, J.F. and Fujasová, M., 2009. Improved power and mass transfer correlations for design and scale-up of multi-impeller gas-liquid contactors. Chemical Engineering Science, 64(3), pp.598-604.

Moucha, T., Linek, V., \& Prokopová, E., 2003. Gas hold-up, mixing time and gas-liquid volumetric mass transfer coefficient of various multiple-impeller configurations: Rushton turbine, pitched blade and techmix impeller and their combinations. Chemical Engineering Science 58(9), pp. 1839-1846.

Murthy, B. N., Ghadge, R. S., \& Joshi, J. B., 2007. CFD simulations of gas-liquid-solid stirred reactor: Prediction of critical impeller speed for solid suspension. Chemical Engineering Science, 62(24), pp. 7184-7195.

Nagata, I. and Yamada, T., 1972. Correlation and prediction of heats of mixing of liquid mixtures. Industrial \& Engineering Chemistry Process Design and Development, 11(4), pp.574578.

Nagase, Y., \& Yasui, H., 1983. Fluid motion and mixing in a gas-liquid contactor with turbine agitators. The Chemical Engineering Journal, 27(1), pp. 37-47.

Nere, N. K., Patwardhan, A. W., \& Joshi, J. B., 2003. Liquid-phase mixing in stirred vessels: turbulent flow regime. Industrial \& engineering chemistry research, 42(12), pp. 2661-2698.

Nienow, A. W., 1998. Hydrodynamics of stirred bioreactors. Applied Mechanics Reviews 51(1), pp. 3-32.

Nienow, A. W., \& Bujalski, W., 2004. The versatility of up-pumping hydrofoil agitators. Chemical Engineering Research and Design, 82(9), pp. 1073-1081.

Nienow, A. W., Wisdom, D. J., \& Middleton, J. C., 1977. The effect of scale and geometry on flooding, recirculation and power in gassed stirred vessels. In Proceedings of the 2 nd European Conference on Mixing (p. 1).

Nienow, A. W., \& Wisdom, D. J., 1974. Flow over disc turbine blades. Chemical Engineering Science, 29 (9), pp. 1994-1997. 
Nienow, A.W. and Ulbrecht, J.J., 1985. Gas-liquid mixing and mass transfer in high viscosity liquids. Mixing of liquids by mechanical agitation. Gordons and Breach, New York, pp.203-235.

Nienow, A.W. and Elson, T.P., 1988. Aspects of mixing in rheologically complex fluids. Chemical engineering research \& design, 66(1), pp.5-15.

Nienow, A. W., 1990. Agitators for mycelial fermentations. Trends in biotechnology, 8, pp. 224233.

Nienow, A.W., Hunt, G. and Buckland, B.C., 1996. A fluid dynamic study using a simulated viscous, shear thinning broth of the retrofitting of large agitated bioreactors. Biotechnology and bioengineering, 49(1), pp.15-19.

Nocentini, M., Fajner, D., Pasquali, G. and Magelli, F., 1993. Gas-liquid mass transfer and holdup in vessels stirred with multiple Rushton turbines: water and water-glycerol solutions. Industrial \& engineering chemistry research, 32(1), pp.19-26.

Novak, V. and Rieger, F., 1975. Homogenization efficiency of helical ribbon and anchor agitators. The Chemical Engineering Journal, 9(1), pp.63-70.

Otomo, N., Bujalski, W. and Nienow, A.W., 1995. The application of a compartment model to a vessel stirred with either dual radial or dual axial flow impellers. The 1995 Institute of Chemical Engineers Research Event, pp.829-831.

Pacek, A.W., Man, C.C. and Nienow, A.W., 1998. On the Sauter mean diameter and size distributions in turbulent liquid/liquid dispersions in a stirred vessel. Chemical Engineering Science, 53(11), pp.2005-2011.

Pakzad, L., Ein-Mozaffari, F., Upreti, S.R. and Lohi, A., 2013a. Evaluation of the mixing of nonNewtonian biopolymer solutions in the reactors equipped with the coaxial mixers through tomography and CFD. Chemical engineering journal, 215, pp.279-296.

Pakzad, L., Ein-Mozaffari, F., Upreti, S.R. and Lohi, A., 2013b. A novel and energy-efficient coaxial mixer for agitation of non-Newtonian fluids possessing yield stress. Chemical Engineering Science, 101, pp.642-654.

Pakzad, L., Ein-Mozaffari, F., Upreti, S.R., \& Lohi, A., 2013c. Using tomography to assess the efficiency of the coaxial mixers in agitation of yield-pseudoplastic fluids. Chemical Engineering Research and Design 91(9), pp. 1715-1724.

Pakzad, L., Ein-Mozaffari, F., Upreti, S.R. and Lohi, A., 2013d. Agitation of Herschel-Bulkley fluids with the Scaba-anchor coaxial mixers. Chemical Engineering Research and Design, 91(5), pp.761-777. 
Pandit, A. B., \& Joshi, J. B., 1983. Mixing in mechanically agitated gas-liquid contactors, bubble columns and modified bubble columns. Chemical Engineering Science, 38(8), pp. 1189-1215.

Pant, H. J., Kundu, A., \& Nigam, K. D. P., 2001. Radiotracer applications in chemical process industry. Reviews in Chemical Engineering, 17 (3), pp. 165-252.

Patel, D., Ein-Mozaffari, F., \& Mehrvar, M., 2014. Tomography images to analyze the deformation of the cavern in the continuous-flow mixing of non-Newtonian fluids. AIChE Journal 60(1), pp. 315-331.

Patwardhan, A. W., \& Joshi, J. B., 1999. Relation between flow pattern and blending in stirred tanks. Industrial and Engineering Chemistry Research, 38 (8), pp. 3131-3143.

Patwardhan, A. W., \& Gaikwad, S. G., 2003. Mixing in tanks agitated by jets. Chemical Engineering Research and Design, 81(2), pp. 211-220.

Paul, E. L., Atiemo-Obeng, V., \& Kresta, S. M. (Eds.). (2004). Handbook of industrial mixing: science and practice. Wiley. com.

Petitti, M., Nasuti, A., Marchisio, D. L., Vanni, M., Baldi, G., 2010. Bubble Size Distribution Modeling in Stirred Gas-Liquid Reactors with QMOM Augmented by a New Correction Algorithm. AIChE. J. 56(1), 36-53.

Petitti, M., Vanni, M., Marchisio, D.L., Buffo, A. and Podenzani, F., 2013. Simulation of coalescence, break-up and mass transfer in a gas-liquid stirred tank with CQMOM. Chemical engineering journal, 228, pp.1182-1194.

Pinelli, D., Nocentini, M. and Magelli, F., 1994. Hold-up in low viscosity gas-liquid systems stirred with multiple impellers. Comparison of different agitators types and sets. In Institution of Chemical Engineers Symposium Series (Vol. 136, pp. 81-81). HEMSPHERE PUBLISHING CORPORATION.

Prince, M.J. and Blanch, H.W., 1990. Bubble coalescence and break-up in air-sparged bubble columns. AIChE Journal, 36(10), pp.1485-1499.

Raghav Rao, K. S. M. S., \& Joshi, J. B., 1988. Liquid phase mixing in mechanically agitated vessels. Chemical Engineering Communications, 74 (1), pp. 1-25.

Ranganathan, P. and Sivaraman, S., 2011. Investigations on hydrodynamics and mass transfer in gas-liquid stirred reactor using computational fluid dynamics. Chemical engineering science, 66(14), pp.3108-3124.

Ranade, V.V., 2002. Preface. Process Systems Engineering, 5, pp.xi-xiii. 
Ranade, V. V., \& Deshpande, V. R., 1999. Gas-liquid flow in stirred reactors: trailing vortices and gas accumulation behind impeller blades. Chemical Engineering Science, 54(13), pp. 2305-2315.

Razzak, S.A., Barghi, S. and Zhu, J.X., 2009. Application of electrical resistance tomography on liquid-solid two-phase flow characterization in an LSCFB riser. Chemical Engineering Science, 64(12), pp.2851-2858.

Razzak, S.A., Barghi, S. and Zhu, J.X., 2010. Axial hydrodynamic studies in a gas-liquid-solid circulating fluidized bed riser. Powder Technology, 199(1), pp.77-86.

Rewatkar, V. B., Rao, K. R., \& Joshi, J. B., 1991. Critical impeller speed for solid suspension in mechanically agitated three-phase reactors. 1. Experimental part. Industrial and Engineering Chemistry Research, 30 (8), pp. 1770-1784.

Rieger, F., Novák, V., \& Havelková, D., 1986. Homogenization efficiency of helical ribbon agitators. The Chemical Engineering Journal, 33(3), pp. 143-150.

Reilly, C.D. and Britter, R.E., 1985. Mixing times for passive tracers in stirred tanks.

Van't Riet, K. and Smith, J.M., 1975. The trailing vortex system produced by Rushton turbine agitators. Chemical Engineering Science, 30(9), pp.1093-1105.

Rivera, C. A., Heniche, M., Takenaka, K., \& Tanguy, P. A., 2009. Finite element modeling of the laminar and transition flow of the Superblend dual shaft coaxial mixer on parallel computers. Chemical Engineering Science, 64(21), pp. 4442-4456.

Roustan, M., 1985, June. Power consumed by Rushton turbines in non standard vessels under gassed conditions. In Proceedings of the 5th European Conference on Mixing, Wurzburg, Germany.

Rushton, J.H., Costich, E.W. and Everett, H.J., 1950. Power characteristics of mixing impellers. 1. Chemical Engineering Progress, 46(8), pp.395-404.

Rushton, J. H., \& Bmbinet, J. J., 1968. Holdup and flooding in air liquid mixing. The Canadian Journal of Chemical Engineering, 46(1), pp. 16-21.

Rudolph, L., Schäfer, M., Atiemo-Obeng, V., \& Kraume, M., 2007. Experimental and numerical analysis of power consumption for mixing of high viscosity fluids with a co-axial mixer. Chemical Engineering Research and Design, 85 (5), pp. 568-575.

Saffman, P. G., \& Turner, J. S., 1956. On the collision of drops in turbulent clouds. Journal of Fluid Mechanics, 1, 16. 
Sarkar, J., Shekhawat, L.K., Loomba, V. and Rathore, A.S., 2016. CFD of mixing of multi-phase flow in a bioreactor using population balance model. Biotechnology progress. DOI $10.1002 /$ btpr.2242

Sato, Y. and Sekoguchi, K., 1975. Liquid velocity distribution in two-phase bubble flow. International Journal of Multiphase Flow, 2(1), pp.79-95.

Scargiali, F., D'Orazio, A., Grisafi, F. and Brucato, A., 2007. Modelling and simulation of gasliquid hydrodynamics in mechanically stirred tanks. Chemical Engineering Research and Design, 85(5), pp.637-646.

Schäfer, M., Wächter, P. and Durst, F., 2000, July. Experimental investigation of local bubble size distributions in stirred vessels using phase Doppler anemometry. In 10th European Conference on Mixing (pp. 205-212). Elsevier, Delft, The Netherlands.

Scott, D. M., McCann, H., 2005. Process imaging for automatic control. CRC Press.

Sharifi, M. and Young, B., 2013. Electrical resistance tomography (ERT) applications to chemical engineering. Chemical Engineering Research and Design, 91(9), pp.1625-1645.

Shaw, J.A., 1994. Understand the effects of impeller type, diameter, and power on mixing time. Chemical engineering progress, 90(2), pp.45-48.

Shewale, S. D., \& Pandit, A. B., 2006. Studies in multiple impeller agitated gas-liquid contactors. Chemical engineering science, 61(2), pp. 489-504.

Shiue, S.J. and Wong, C.W., 1984. Studies on homogenization efficiency of various agitators in liquid blending. The Canadian Journal of Chemical Engineering, 62(5), pp.602-609.

Silva, E. M., Rogez, H., \& Larondelle, Y., 2007. Optimization of extraction of phenolics from Inga edulis leaves using response surface methodology. Separation and Purification Technology, 55(3), pp. 381-387.

Supardan, M. D., Masuda, Y., Maezawa, A., \& Uchida, S., 2004. Local gas holdup and mass transfer in a bubble column using an ultrasonic technique and a neural network. Journal of chemical engineering of Japan, 37(8), pp. 927-932.

Taghavi, M., Zadghaffari, R., Moghaddas, J., \& Moghaddas, Y., 2011. Experimental and CFD investigation of power consumption in a dual Rushton turbine stirred tank. Chemical Engineering Research and Design, 89(3), pp. 280-290.

Takashi, K., Arai, K., \& Saito, S., 1980. Power correlation for anchor and helical ribbon impellers in highly viscous liquids. Journal of Chemical Engineering of Japan, 13(2), pp. 147-150. 
Takahashi, K. and Nienow, A.W., 1993. Bubble sizes and coalescence rates in an aerated vessel agitated by a Rushton turbine. Journal of chemical engineering of Japan, 26(5), pp.536-542.

Thatte, A. R., Ghadge, R. S., Patwardhan, A. W., Joshi, J. B., \& Singh, G., 2004. Local gas holdup measurement in sparged and aerated tanks by $\gamma$-ray attenuation technique. Industrial \& engineering chemistry research, 43(17), pp. 5389-5399.

Thibault, F., Tanguy, P. A., 2002. Power-draw analysis of a coaxial mixer with Newtonian and non-Newtonian fluids in the laminar regime. Chemical Engineering Science 57(18), pp. 38613872.

Tomiyama, A., 1998. Struggle with computational bubble dynamics. Multiphase Science and Technology, 10(4), pp.369-405.

Utomo, M. B., Sakai, T., Uchida, S., \& Maezawa, A., 2001. Simultaneous measurement of mean bubble diameter and local gas holdup using ultrasonic method with neural network. Chemical engineering \& technology, 24(5), pp. 493-500.

Van't Riet, K., 1976. Power consumption, impeller coalescence and recirculation in aerated vessels. Transactions of the Institution of Chemical Engineers, 54, pp. 124-131.

Vasconcelos, J.M., Orvalho, S.C., Rodrigues, A.M. and Alves, S.S., 2000. Effect of blade shape on the performance of six-bladed disk turbine impellers. Industrial \& engineering chemistry research, 39(1), pp.203-213.

Vasconcelos, J. M., Alves, S. S., \& Barata, J. M., 1995. Mixing in gas-liquid contactors agitated by multiple turbines. Chemical engineering science, 50(14), 2343-2354.

Venneker, B.C., Derksen, J.J. and Van den Akker, H.E., 2002. Population balance modeling of aerated stirred vessels based on CFD. AIChE Journal, 48(4), pp.673-685.

Vlachakis, V.N., 2006. Turbulent characteristics in stirring vessels: a numerical investigation. http://hdl.handle.net/10919/34599

Vlaev, S.D. and Martinov, M., 1998. Non-uniformity of gas dispersion in turbine-generated viscoelastic circulation flow. The Canadian Journal of Chemical Engineering, 76(3), pp.405-412.

Vrabel, P., van der Lans, R. G., Luyben, K. C. A., Boon, L., \& Nienow, A. W., 2000. Mixing in large-scale vessels stirred with multiple radial or radial and axial up-pumping impellers: modelling and measurements. Chemical engineering science, 55(23), pp. 5881-5896.

Wachi, S., Morikawa, H. and Ueyama, K., 1987. Gas holdup and axial dispersion in gas-liquid concurrent bubble column. Journal of chemical engineering of Japan, 20(3), pp.309-316. 
Wang, H., Jia, X., Wang, X., Zhou, Z., Wen, J. and Zhang, J., 2014. CFD modeling of hydrodynamic characteristics of a gas-liquid two-phase stirred tank. Applied Mathematical Modelling, 38(1), pp.63-92.

Wang, M., Dorward, A., Vlaev, D., \& Mann, R., 2000. Measurements of gas-liquid mixing in a stirred vessel using electrical resistance tomography (ERT). Chemical Engineering Journal 77(1), pp. 93-98.

Wang, W., Mao, Z. S., \& Yang, C., 2006. Experimental and numerical investigation on gas holdup and flooding in an aerated stirred tank with Rushton impeller. Industrial \& engineering chemistry research, 45(3), pp. 1141-1151.

Warsito, W., \& Fan, L. S., 2001. Measurement of real-time flow structures in gas-liquid and gasliquid-solid flow systems using electrical capacitance tomography (ECT). Chemical Engineering Science, 56(21), pp. 6455-6462.

Yang, S., Li, X., Yang, C., Ma, B. and Mao, Z.S., 2015. Computational Fluid Dynamics Simulation and Experimental Measurement of Gas and Solid Holdup Distributions in a Gas-Liquid-Solid Stirred Reactor. Industrial \& Engineering Chemistry Research, 55(12), pp.3276-3286.

Yawalkar, A. A., Heesink, A. B. M., Versteeg, G. F., \& Pangarkar, V. G., 2002. Gas-Liquid Mass Transfer Coefficient in Stirred Tank Reactors. The Canadian Journal of Chemical Engineering, 80(5), pp. 840-848.

Zahradnık, J., Mann, R., Fialova, M., Vlaev, D., Vlaev, S. D., Lossev, V., \& Seichter, P., 2001. A networks-of-zones analysis of mixing and mass transfer in three industrial bioreactors. Chemical engineering science, 56(2), pp. 485-492.

Zhao, D., Gao, Z., Müller-Steinhagen, H., \& Smith, J. M., 2001. Liquid-phase mixing times in sparged and boiling agitated reactors with high gas loading. Industrial \& engineering chemistry research, 40(6), pp.1482-1487.

Zhu, H., Nienow, A. W., Bujalski, W., \& Simmons, M. J., 2009. Mixing studies in a model aerated bioreactor equipped with an up-or a down-pumping 'Elephant Ear'agitator: Power, hold-up and aerated flow field measurements. Chemical Engineering Research and Design 87(3), pp. 307-317.

Zlokarnik, M. (1988). Stirring. Wiley-VCH Verlag GmbH \& Co. KGaA. 